\title{
Verification And Validation Of TMAP7
}

\author{
James Ambrosek \\ Glen R. Longhurst
}

December 2005

Idaho National Engineering and Environmental Laboratory Bechtel BWXT Idaho, LLC 


\title{
Verification And Validation Of TMAP7
}

\author{
James Ambrosek \\ Glen R. Longhurst
}

December 2005

\section{Idaho National Engineering and Environmental Laboratory Idaho Falls, Idaho 83415}

\author{
Prepared for the \\ U.S. Department of Energy \\ Office of Science \\ Under DOE Idaho Operations Office \\ Contract DE-AC07-05ID14517
}





\begin{abstract}
The Tritium Migration Analysis Program, Version 7 (TMAP7) code is an update of TMAP4, an earlier version that was verified and validated in support of the International Thermonuclear Experimental Reactor (ITER) program and of the intermediate version TMAP2000. It has undergone several revisions. The current one includes radioactive decay, multiple trap capability, more realistic treatment of heteronuclear molecular formation at surfaces, processes that involve surface-only species, and a number of other improvements. Prior to code utilization, it needed to be verified and validated to ensure that the code is performing as it was intended and that its predictions are consistent with physical reality. To that end, the demonstration and comparison problems cited here show that the code results agree with analytical solutions for select problems where analytical solutions are straightforward or with results from other verified and validated codes, and that actual experimental results can be accurately replicated using reasonable models with this code. These results and their documentation in this report are necessary steps in the qualification of TMAP7 for its intended service. This revision updates results using TMAP7.1, which corrected some code deficiencies found in TMAP7.
\end{abstract}




\section{CONTENTS}

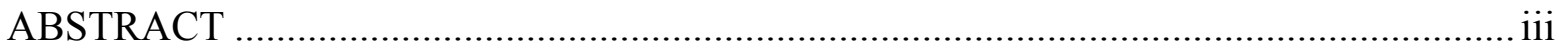

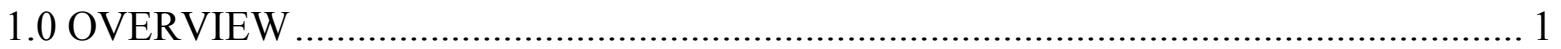

2.0 SPECIALIZED PROBLEMS ........................................................................ 2

2.1 Problem 1a: Diffusion from a Depleting Source (Val-1a)............................................2

2.2 Problem 1b: Diffusion in a Semi-Infinite Slab with Constant-Source Boundary

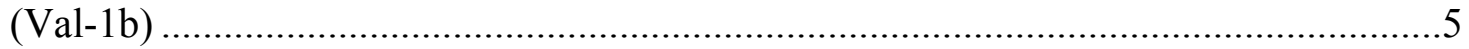

2.3 Problem 1c: Diffusion in a Partially Preloaded Semi-Infinite Slab (Val-1c) .................8

2.4 Problem 1d: Permeation Problem with Trapping (Val-1da, Val-1db, Val-1dc)............11

2.4.1 Effective Diffusivity Trap (Val-1da) ................................................................12

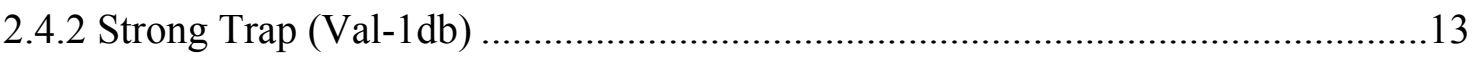

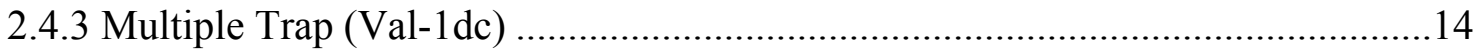

2.5 Problem 1e: Diffusion with Composite Material Layers (Val-1e) ………………........15

2.6 Problem 1f: Heat Sink/Source Problem.....................................................................18

2.6.1 Heat conduction with generation (Val-1fa) ......................................................18

2.6.2 Thermal Diffusion Transient (Val-1fb) ............................................................19

2.6.3 Conduction in Composite Structure with Constant Surface Temperatures

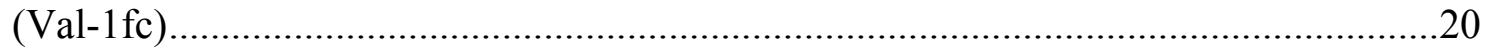

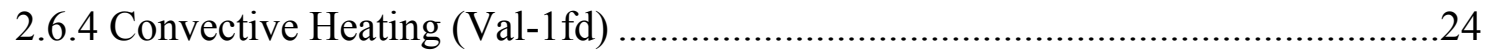

2.7 Problem 1g: Enclosure Reaction Problems ...........................................................25

2.7.1 Simple Forward Reactions (Val-1ga and Val-1gb) ...........................................26

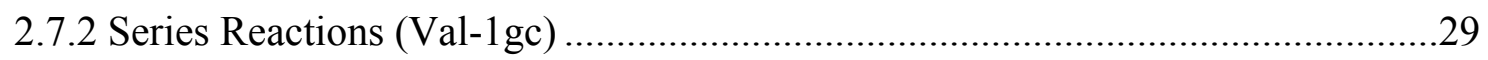

2.8 Problem 1h: Flow Through Multiple Enclosures ……………………….................32

2.8.1 Three Enclosure Problem (Val-1ha) ..................................................................

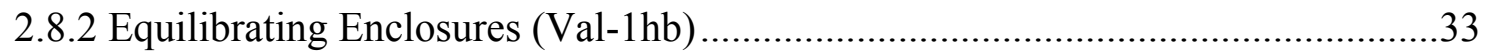

2.9 Problem 1i: Species Equilibration on a Reactive Surface ……………………….........37

2.9.1 Ratedep Conditions (Val-1ia, Val-1ib) ..............................................................

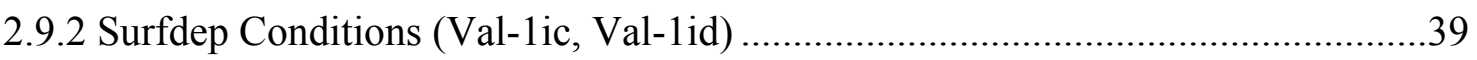

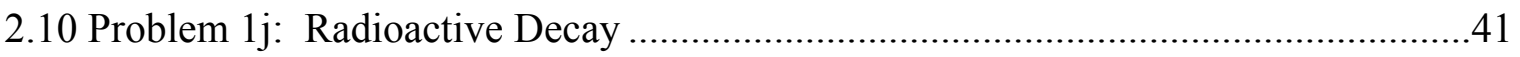

2.10.1 Problem 1ja: Radioactive Decay of Mobile Tritium in a Slab (Val-1ja).............41

2.10.2 Problem 1jb: Decay of Tritium in a Distributed Trap (Val-1jb) .........................43

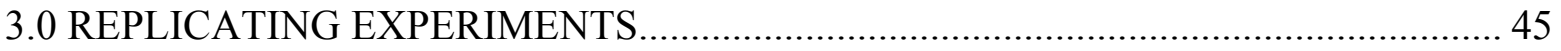

3.1 Problem 2a: Ion Implantation Experiment (Val-2a) ...................................................45 
3.2 Problem 2b: Diffusion Experiment in Beryllium (Val-2ba, Val-2bb).........................46

3.3 Problem 2c: Test Cell Release Experiment (Val-2c)...................................................48

3.4 Problem 2d. Thermal Desorption Spectroscopy on Tungsten (Val-2d) ........................49

3.5 Problem 2e. Co-permeation of $\mathrm{H}_{2}$ and $\mathrm{D}_{2}$ through Pd (Val-2e) .................................53

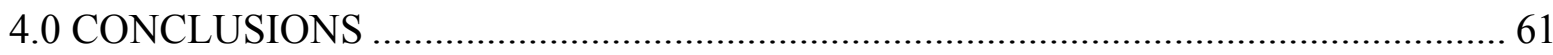

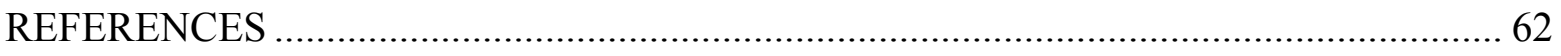

APPENDIX A SPECIES EQUILIBRATION MODEL ……......................................... A-1

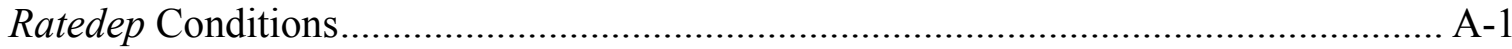

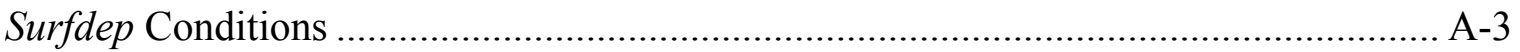

APPENDIX B PROBLEM INPUT FILE LISTINGS ….................................................. B-1

\section{FIGURES}

Figure 1. Fractional release of tritium from an enclosure through $\mathrm{SiC}$ in depleting source demonstration problem (Val-1a)..............................................................................

Figure 2. Atom flux through outside face of membrane for depleting source problem (Val1a).

Figure 3. Concentration profile in a semi-infinite slab of $\mathrm{SiC}$ after $25 \mathrm{~s}$ from problem Val$1 \mathrm{~b}$

Figure 4. Effective-diffusivity, single trap (Val-1da, Val-1da1). ..............................................13

Figure 5. Permeation for strong-trapping regime (Val-1db).....................................................14

Figure 6. Permeation curve for slab with multiple traps (Val-1dc). ..........................................15

Figure 7. Comparison of TMAP7 with theoretical solution for transient concentration at a location $7.5 \mu \mathrm{m}$ deep in the $\mathrm{SiC}$ layer of a composite slab of $\mathrm{PyC}$ and $\mathrm{SiC}$ (Val-1e).

Figure 8. Transient temperature distribution for various times in a slab (Val-1 fb) ......................20

Figure 9. Convective heating at depth $5 \mathrm{~cm}$ in a semi-infinite slab (Val-1fd)............................25

Figure 10. Production of $A B$ from $A$ and $B$ under assumptions of equal and unequal initial reactant concentrations (Val-1ga/Val-1gb).

Figure 11. Partial pressures of species in series reaction (Val-1gc)...........................................32

Figure 12. Concentration history of sequentially coupled enclosures (Val-1ha)...........................35

Figure 13. Tritium concentration equilibration in two communicating enclosures (Val-1hb).

Figure 14. Equilibration of $\mathrm{H}_{2}$ with $\mathrm{D}_{2}$ to form $\mathrm{HD}$ on a tungsten surface under the assumption of equal starting partial pressures for the reactants and ratedep boundary conditions (Val-1ia). 
Figure 15. Chemical equilibration of $\mathrm{H}_{2}$ and $\mathrm{D}_{2}$ to $\mathrm{HD}$ on a tungsten surface with unequal starting partial pressures and ratedep boundary conditions (Val-1ib).

Figure 16. Chemical equilibration of $\mathrm{H}_{2}$ and $\mathrm{D}_{2}$ to form $\mathrm{HD}$ under surfdep boundary conditions with equal starting pressures (Val-1ic)......

Figure 17. Chemical equilibration of $\mathrm{H}_{2}$ and $\mathrm{D}_{2}$ to form HD under surfdep boundary conditions with unequal starting pressures (Val-1id).

Figure 18. Decay of mobile tritium and associated growth of ${ }^{3} \mathrm{He}$ in a diffusion segment (Val-1ja).

Figure 19. Profiles of trapped tritium at the beginning and end of a 45-year decay and the profile of the resultant ${ }^{3} \mathrm{He}$ at the end of that time (Val-1jb).

Figure 20. Loss of trapped tritium by radioactive decay is reflected in the gain of He-3 (Val-1jb).

Figure 21. Plasma Driven Permeation of PCA (Val-2a).....................................................46

Figure 22. Thermal desorption test of beryllium (Val-2b) .....................................................48

Figure 23. HTO Concentration in TSTA Exposure Chamber (Val-2c)...................................49

Figure 24. Schematic of system used to model experiments of Hino et al. ${ }^{23}$.............................51

Figure 25. Comparison of calculated with experimental results for Hino's experiment with implantation and thermal desorption of tungsten (Val-2d) ....................................52

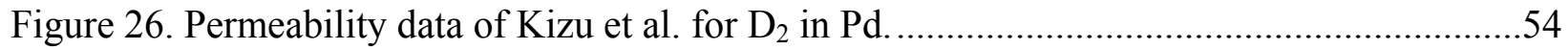

Figure 27. TMAP7 model of experimental system of Kizu et al..........................................55

Figure 28. Comparison of TMAP7 permeation calculations with permeation data of Kizu et al. for $\mathrm{D}_{2}$ only under lawdep boundary conditions using the solubility of Eq. (71) (Val-2ea, Val-2eb, Val-2ec).

Figure 29. Comparison of TMAP7 results using a lawdep boundary condition on each side of the membrane wirh the experiment s of Kizu et al. (Val-2ed) .58

Figure 30. Comparison of TMAP7 calculation with simple ratedep boundary conditions with the values measured by Kizu et al. (Val-2eea).

Figure 31. TMAP7 results from a 5-fold reduction in $K_{d}$ and $K_{r}$ (Val-2eeb)

Figure 32. Comparison of TMAP7 calculation for lawdep boundary condition upstream and ratedep boundary condition downstream with measurements made by Kizu et al. (Val-2ef).

\section{TABLES}

Table 1. Fractional release of tritium from depleting source problem Val-1a.............................3

Table 2. Concentration profile at $x=0.15 \mathrm{~m}$ for problem Val-1b, diffusion in a semiinfinite slab.

Table 3. Concentration profile (atom $/ \mathrm{m}^{3}$ ) at $t=25 \mathrm{sec}$ for diffusion in a semi-infinite slab. 7 
Table 4. Flux (atom $/ \mathrm{m}^{2} \mathrm{sec}$ ) into semi-infinite slab from a constant source................................8

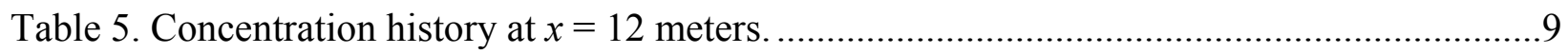

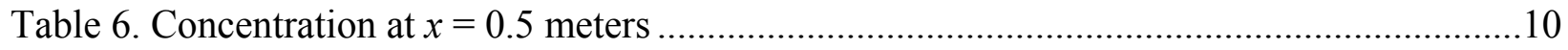

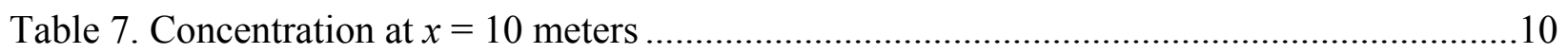

Table 8. Steady-State Concentration Profile in Composite Slab .............................................. 16

Table 9. Variance for transient solution in composite slab..................................................... 17

Table 10. Heat Conduction with Generation ........................................................................19

Table 11. Temperature distribution in composite structure at $t=150$ seconds.........................21

Table 12. Temperature history in composite structure at $x=0.09$ meters ..............................22

Table 13. Steady-state temperature $(\mathrm{K})$ distribution for composite structure .............................23

Table 14. Heating of Semi-Infinite Slab by Convection...........................................................25

Table 15. Partial pressure $(\mathrm{Pa})$ of product for equal starting concentrations.............................27

Table 16. Partial pressure $(\mathrm{Pa})$ of product for reaction with unequal starting

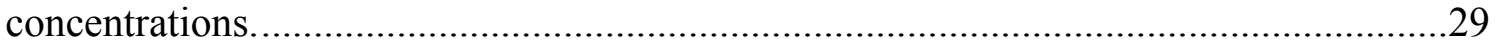

Table 17. Partial pressures of species in a series reaction. .......................................................31

Table 18. Concentration profiles of enclosures 2 and 3 with convective flow..........................33

Table 19. Concentration of tritium in convective flow between two enclosures...........................36

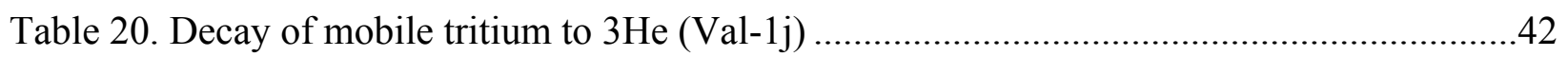




\subsection{OVERVIEW}

The TMAP Code was written at the Idaho National Engineering and Environmental Laboratory by Brad Merrill and James Jones in the late 1980s as a tool for safety analysis of systems involving tritium. ${ }^{1}$ Since then it has been upgraded to TMAP4 and has been used in numerous applications including experiments supporting fusion safety, predictions for advanced systems such as the International Thermonuclear Experimental Reactor (ITER), and estimates involving tritium production technologies. The code's further upgrade to TMAP2000 ${ }^{2}$ and then to TMAP7 was accomplished in response to several needs. TMAP and TMAP4 had the capacity to deal with only a single trap for diffusing gaseous species in solid structures. TMAP7 includes up to three separate traps and up to 10 diffusing species. The original code had difficulty dealing with heteronuclear molecule formation such as HD. That has been removed. Under solution-law dependent diffusion boundary conditions, such as Sieverts' law, TMAP7 automatically generates heteronuclear molecular partial pressures and surface flows when solubilities and partial pressures of the homonuclear molecular species are provided. A further sophistication is the addition of non-diffusing surface species. Atoms such as oxygen or nitrogen or complexes such as hydroxyl radicals on metal surfaces are sometimes important in molecule formation with diffusing hydrogen isotopes but do not themselves diffuse appreciably in the material. TMAP7 will accommodate up to 30 such surface species, allowing the user to specify relationships between those surface concentrations and partial pressures of gaseous species above the surfaces or to form them dynamically by combining diffusion species or other surface species. Additionally, TMAP7 allows the user to include a surface binding energy and an adsorption barrier energy and includes asymmetrical diffusion between the surface sites and regular diffusion sites in the bulk. All of the previously existing features for heat transfer, flows between enclosures, and chemical reactions within the enclosures have been retained, but the allowed problem size and complexity have been increased to take advantage of the greater memory and speed available on modern computers. One feature unique to TMAP7 is the addition of radioactive decay for both trapped and mobile species. Another is the ability to initialize distributed parameters such as initial mobile atom, trapped atom, or trap concentrations using selected mathematical functions. Also, time-dependent temperatures and pressures can be specified in boundary enclosures and for surface concentrations of diffusion species.

Since its release in November 2004, TMAP7 was found to have a few minor errors that prevented correct results from being obtained in certain problems. Those errors have been corrected in TMAP7.1, which will not be specifically referred to hereafter except as TMAP7. Results included in this revision are for the TMAP7.1 version of the code.

The verification and validation process normally involves two steps. The verification process is a careful examination of the code to ensure that the coding faithfully reproduces the mathematical model and that the code is well written and efficient. That process was pursued extensively with TMAP4 but has not been done independently of code development here. The basic architecture of the code remains the same, although a number of minor changes were required to work with the GNU FORTRAN 77, selected for distribution with the code. There are also new components and a few new subroutines. These have been carefully evaluated for coding accuracy, but the demonstration of their success is in the high fidelity the code provides to the sample problems. Those sample problems constitute the validation of the code and provide the basis for what is presented here. 
There are two main sections to this report. The first exercises TMAP7 in each of its major capability areas using specialized problems, showing that the results computed by TMAP7 are in good agreement with "known" results. This demonstrates that the code's functional tools are performing properly. The second part of the report provides a comparison of TMAP7 results with experimental results to show the general utility of the code in modeling reality.

\subsection{SPECIALIZED PROBLEMS}

Computational capabilities of TMAP7 lie in six major areas: diffusion and trapping within structures and surface processes, heat transfer, chemical reactions in enclosures, bulk fluid flows, chemical equilibrium and radioactive decay. The demonstration problems that follow are grouped into those areas.

Problems 1a-1e exercise TMAP7's mass transfer capabilities

Problems 1f (a-c) demonstrate TMAP7's heat transfer functions

Problems 1g (a-c) model enclosure reactions

Problems 1h (a-b) deal with enclosure flow

Problem 1i (a-b) verify chemical reactions in enclosures and on surfaces are correct

Problem $1 \mathrm{j}$ demonstrates radioactive decay.

The descriptions of these problems include a statement of the problem, a description of the modeling used in setting up the problem for TMAP7, and a comparison of the TMAP7 results with "known" solutions from literature or other sources. Appendix A is the derivation for the surface equilibrium model used in problem 1i (b). Appendix B contains the input code listings for each of the problems cited in the report.

The file names assigned to the various problems appear in parentheses in the headings for the problem descriptions. Input files carry the inp extension, output or codeout files have .out, and plot data files (pltdata) terminate with the .plt extension.

Theoretical results were calculated using Microsoft ExcelTM, and TMAP7 calculations were obtained in two working environments. One used Windows XPTM on a Dell Optiplex GX 260 and on a custom-built Pentium 4 3.0-GHz machine. The other was Windows $\mathrm{ME}^{\mathrm{TM}}$ running on a Dell Dimension XPS R450 and on a Dell Latitude C-600 laptop computer.

\subsection{Problem 1a: Diffusion from a Depleting Source (Val-1a)}

This diffusion problem models an enclosure that is pre-charged with a fixed quantity of tritium. At time $t>0$, the tritium is allowed to diffuse through a finite slab of SiC, initially at zero concentration. The surface of the slab in contact with the source is assumed to be in equilibrium with the source enclosure. The boundary condition at the exit side of the slab is kept constant at zero concentration for all time. The concentration of the enclosure is then calculated for different times and reported as a fractional release. There are no trapping effects active in the slab.

Carslaw and Jaeger ${ }^{3}$ give the analytical solution for an analogous heat transfer problem from which the solute concentration profile in the membrane is 


$$
C(x, t)=2 S P_{0} L \sum_{n=1}^{\infty} \frac{\exp \left(-\alpha_{n}^{2} D t\right) \sin \left(\alpha_{n} x\right)}{\left[l\left(\alpha_{n}^{2}+L^{2}\right)+L\right] \sin \left(\alpha_{n} l\right)}
$$

where

$$
\begin{aligned}
& \alpha_{n}=\frac{L}{\tan \left(\alpha_{n} l\right)} \\
& L=\frac{S T A k}{V}
\end{aligned}
$$

Here

$A=$ cross-sectional area of the slab $\left(2.16 \times 10^{-6} \mathrm{~m}^{2}\right)$

$\mathrm{D}=$ diffusivity of tritium ( $\mathrm{SiC}$ assumed: $2.62238 \mathrm{E}-11 \mathrm{~m}^{2} / \mathrm{s}$ at $2373 \mathrm{~K}$ )

$k=$ Boltzmann's constant $\left(1.38065 \times 10^{-23} \mathrm{~J} / \mathrm{K}\right)$

$l=$ thickness of the slab $\left(3.30 \times 10^{-5} \mathrm{~m}\right)$

$S=$ solubility of tritium ( $\mathrm{SiC}$ assumed: $7.244 \mathrm{E} 22 / \mathrm{T}$ atom $/ \mathrm{m}^{3} / \mathrm{Pa}$ )

$T=$ temperature $(2373 \mathrm{~K})$

$V=$ volume of the enclosure $\left(5.20 \times 10^{-11} \mathrm{~m}^{3}\right)$

We apply Henry's law to the concentration at $x=l$ to find the gas pressure in the enclosure

$$
P(t)=\frac{C(l, t)}{S}=2 P_{0} L \sum_{n=1}^{\infty} \frac{\exp \left(-\alpha_{n}^{2} D t\right)}{l\left(\alpha_{n}^{2}+L^{2}\right)+L}
$$

and finally the release fraction

$$
F R=1-\frac{P(t)}{P_{0}}=1-2 L \sum_{n=1}^{\infty} \frac{\exp \left(-\alpha_{n}^{2} D t\right)}{l\left(\alpha_{n}^{2}+L^{2}\right)+L}
$$

Some of the values obtained from Equation (5) and from TMAP7 are compared in Table 1. Ten terms were included in the sum of (5) so that even at $t=1 \mathrm{~s}$, the last term was less than $10^{-10}$ of the sum. The variance between the analytical solution and the computed solution from TMAP7 is defined by Equation (6)

$$
\text { Variance }=\frac{T M A P 7-\text { Analytical }}{\text { Analytical }}
$$

Table 1. Fractional release of tritium from depleting source problem Val-1a.

\begin{tabular}{rrrr}
\hline Time (s) & TMAP7 & Theory & Variance \\
\hline 0 & 0.00000 & 0.00000 & 0.00000 \\
1 & 0.19383 & 0.20439 & 0.05169 \\
2 & 0.26151 & 0.26640 & 0.01837 \\
3 & 0.30708 & 0.31022 & 0.01011 \\
4 & 0.34181 & 0.34409 & 0.00662 \\
5 & 0.36998 & 0.37172 & 0.00467
\end{tabular}




\begin{tabular}{rrrr}
\hline Time (s) & TMAP7 & Theory & Variance \\
\hline 6 & 0.39375 & 0.39510 & 0.00342 \\
7 & 0.41434 & 0.41541 & 0.00257 \\
8 & 0.43260 & 0.43343 & 0.00191 \\
9 & 0.44906 & 0.44971 & 0.00144 \\
10 & 0.46415 & 0.46464 & 0.00105 \\
11 & 0.47815 & 0.47851 & 0.00076 \\
12 & 0.49127 & 0.49153 & 0.00054 \\
13 & 0.50368 & 0.50386 & 0.00036 \\
14 & 0.51550 & 0.51562 & 0.00023 \\
15 & 0.52683 & 0.52690 & 0.00013 \\
16 & 0.53774 & 0.53777 & 0.00006 \\
17 & 0.54827 & 0.54827 & 0.00001 \\
18 & 0.55848 & 0.55845 & -0.00005 \\
19 & 0.56839 & 0.56834 & -0.00008 \\
20 & 0.57802 & 0.57797 & -0.00010 \\
21 & 0.58740 & 0.58734 & -0.00011 \\
22 & 0.59655 & 0.59648 & -0.00012 \\
23 & 0.60547 & 0.60540 & -0.00012 \\
24 & 0.61419 & 0.61411 & -0.00013 \\
25 & 0.62270 & 0.62261 & -0.00013 \\
\hline
\end{tabular}

The variance decreases almost monotonically for $t>25 \mathrm{~s}$. Figure 1 shows the comparison for the first $140 \mathrm{~s}$.

A further comparison may be made by noting that the surface flux at $x=0$ is

$$
J=\left.D \frac{\partial C(x, t)}{\partial x}\right|_{x=0}=2 S P_{0} L D \sum_{n=1}^{\infty} \frac{\exp \left(-\alpha_{n}^{2} D t\right) \alpha_{n}}{\left[l\left(\alpha_{n}^{2}+L^{2}\right)+L\right] \sin \left(\alpha_{n} l\right)}
$$

A comparison of results for flux through the free surface is shown in Figure 2.

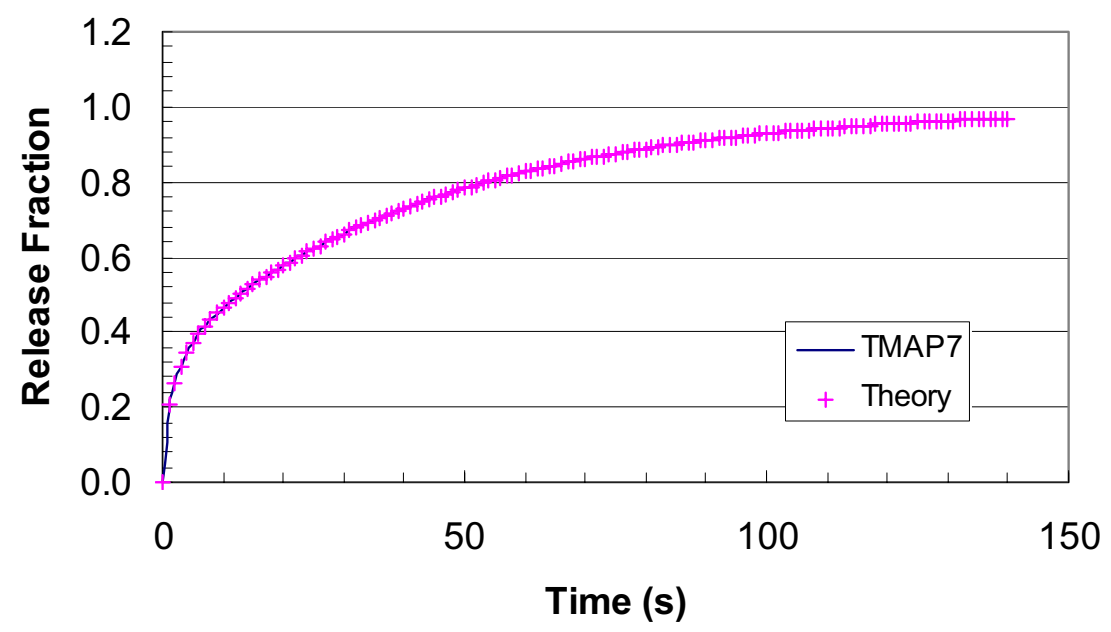

Figure 1. Fractional release of tritium from an enclosure through $\mathrm{SiC}$ in depleting source demonstration problem (Val-1a). 


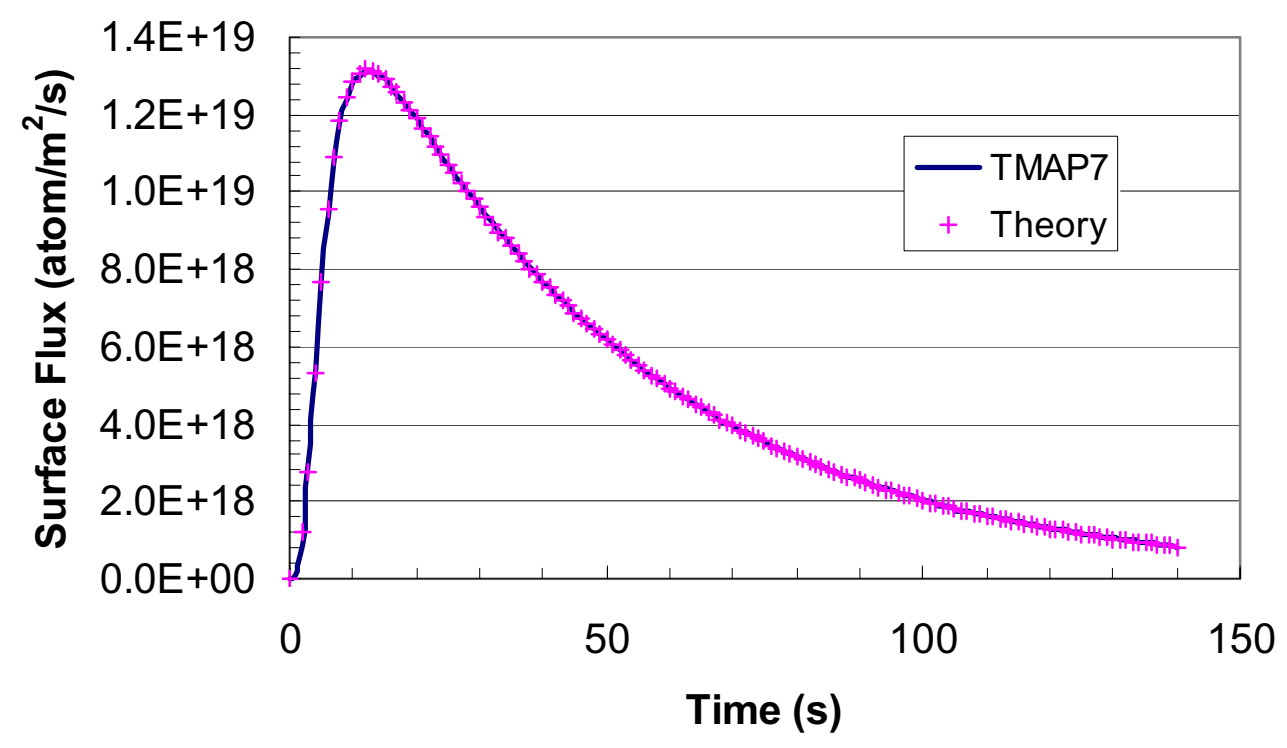

Figure 2. Atom flux through outside face of membrane for depleting source problem (Val-1a).

\subsection{Problem 1b: Diffusion in a Semi-Infinite Slab with Constant-Source Boundary (Val-1b)}

This model is designed to test the basic Fick's-law diffusion. A semi-infinite slab is defined with a constant concentration boundary condition. The initial concentration of the slab is zero for time, $t \leq 0$ seconds. At time $\mathrm{t}>0$, the diffusion is allowed to proceed. The slab is assumed to have no traps. Three comparisons are shown; a transient concentration history at a given location, a spatial concentration profile at a given time, and the variation of flux into the slab surface. These are compared with analytical results.

Carslaw and Jaeger ${ }^{4}$ give the analytical solution to the time-dependent concentration profile as

$$
C(x, t)=C_{o} \operatorname{erfc}\left(\frac{x}{2 \sqrt{D t}}\right) .
$$

where

$C(x, t)=$ diffusion species concentration at position $x$ and time $t$

$C_{o}=$ concentration of the diffusing species at the free surface $\left(1.0\right.$ atoms $\left./ \mathrm{m}^{3}\right)$

$D=\operatorname{diffusivity}\left(1.0 \mathrm{~m}^{2} / \mathrm{s}\right)$.

The solution of Equation (8) was found using Microsoft Excel using the series expansion given in CRC Standard Mathematical Tables and Formulae ${ }^{5}$. This expansion is

$$
\operatorname{erfc}(x)=1-\operatorname{erf}(x)=1-\frac{2}{\sqrt{\pi}}\left(x-\frac{x^{3}}{3}+\frac{1}{2 !} \frac{x^{5}}{5}-\frac{1}{3 !} \frac{x^{7}}{7}+\frac{1}{4 !} \frac{x^{9}}{9} \ldots\right) .
$$


Enough terms were taken in this expansion to ensure that the last term contributed less than $1.0 \times 10^{-12}$ for all values of the argument.

Two comparisons were made for this model between the values of Equation (8) and results from TMAP7. The first comparison was made for times ranging from $t=0$ to $30 \mathrm{~s}$ at a distance from the surface of $x=0.15 \mathrm{~m}$. The disagreement between Equation (8) and TMAP7 was less than $0.05 \%$ at $t=1 \mathrm{sec}$. The variance decreased with time, declining quickly to $0.001 \%$. These values are listed in Table 2.

Table 2. Concentration profile at $x=0.15 \mathrm{~m}$ for problem Val-1b, diffusion in a semi-infinite slab.

\begin{tabular}{rrrr}
\hline Time (s) & TMAP7 & Theory & Variation 1 \\
\hline 0 & 0.000000 & 0.000000 & 0.000000 \\
1 & 0.915140 & 0.915530 & -0.000426 \\
2 & 0.940080 & 0.940215 & -0.000143 \\
3 & 0.951100 & 0.951170 & -0.000074 \\
4 & 0.957660 & 0.957706 & -0.000048 \\
5 & 0.962130 & 0.962167 & -0.000039 \\
6 & 0.965430 & 0.965461 & -0.000033 \\
7 & 0.968000 & 0.968022 & -0.000023 \\
8 & 0.970070 & 0.970086 & -0.000017 \\
9 & 0.971780 & 0.971796 & -0.000017 \\
10 & 0.973230 & 0.973243 & -0.000014 \\
11 & 0.974480 & 0.974488 & -0.000008 \\
12 & 0.975560 & 0.975574 & -0.000014 \\
13 & 0.976520 & 0.976532 & -0.000012 \\
14 & 0.977380 & 0.977385 & -0.000005 \\
15 & 0.978150 & 0.978152 & -0.000002 \\
16 & 0.978840 & 0.978845 & -0.000005 \\
17 & 0.979470 & 0.979477 & -0.000007 \\
18 & 0.980050 & 0.980055 & -0.000005 \\
19 & 0.980580 & 0.980587 & -0.000007 \\
20 & 0.981070 & 0.981078 & -0.000008 \\
21 & 0.981530 & 0.981534 & -0.000004 \\
22 & 0.981950 & 0.981959 & -0.000009 \\
23 & 0.982350 & 0.982355 & -0.000005 \\
24 & 0.982720 & 0.982727 & -0.000007 \\
25 & 0.983070 & 0.983076 & -0.000006 \\
26 & 0.983400 & 0.983404 & -0.000004 \\
27 & 0.983710 & 0.983714 & -0.000004 \\
28 & 0.984010 & 0.984008 & 0.000002 \\
29 & 0.984280 & 0.984286 & -0.000006 \\
30 & 0.984550 & 0.984550 & 0.000000 \\
\hline & & &
\end{tabular}

The second comparison examined the concentration profile from $x=0.05$ to $19.8 \mathrm{~m}$ at increments of $0.1 \mathrm{~m}$ at time, $t=25 \mathrm{~s}$. The variance between Equation (8) and TMAP7 is small, exceeding $0.1 \%$ only at depths greater than $6 \mathrm{~m}$. The comparison of these values can be seen in Table 3, listing values to $11.9 \mathrm{~m}$, and in Figure 3, out to $19.7 \mathrm{~m}$. 
Table 3. Concentration profile (atom $/ \mathrm{m}^{3}$ ) at $t=25 \mathrm{sec}$ for diffusion in a semi-infinite slab.

\begin{tabular}{rrrr}
\hline $\mathbf{x}(\mathbf{m})$ & TMAP7 & Theory & Variance \\
\hline 0 & $1.00 \mathrm{E}-00$ & 1.000000 & 0.000000 \\
0.5 & $9.38 \mathrm{E}-01$ & 0.943628 & -0.005964 \\
1.1 & $8.71 \mathrm{E}-01$ & 0.876377 & -0.006364 \\
1.7 & $8.05 \mathrm{E}-01$ & 0.810008 & -0.006775 \\
2.3 & $7.40 \mathrm{E}-01$ & 0.744977 & -0.007191 \\
2.9 & $6.77 \mathrm{E}-01$ & 0.681717 & -0.007623 \\
3.5 & $6.16 \mathrm{E}-01$ & 0.620618 & -0.008053 \\
4.1 & $5.57 \mathrm{E}-01$ & 0.562031 & -0.008506 \\
4.7 & $5.02 \mathrm{E}-01$ & 0.506255 & -0.008958 \\
5.3 & $4.49 \mathrm{E}-01$ & 0.453536 & -0.009406 \\
5.9 & $4.00 \mathrm{E}-01$ & 0.404064 & -0.009883 \\
6.5 & $3.54 \mathrm{E}-01$ & 0.357971 & -0.010366 \\
7.1 & $3.12 \mathrm{E}-01$ & 0.315334 & -0.010828 \\
7.7 & $2.73 \mathrm{E}-01$ & 0.276178 & -0.011327 \\
8.3 & $2.38 \mathrm{E}-01$ & 0.240476 & -0.011836 \\
8.9 & $2.06 \mathrm{E}-01$ & 0.208157 & -0.012331 \\
9.5 & $1.77 \mathrm{E}-01$ & 0.179109 & -0.012893 \\
10.1 & $1.51 \mathrm{E}-01$ & 0.153190 & -0.013444 \\
10.7 & $1.28 \mathrm{E}-01$ & 0.130227 & -0.014104 \\
11.3 & $1.08 \mathrm{E}-01$ & 0.110029 & -0.014899 \\
11.9 & $9.09 \mathrm{E}-02$ & 0.092392 & -0.015767 \\
\hline & & &
\end{tabular}

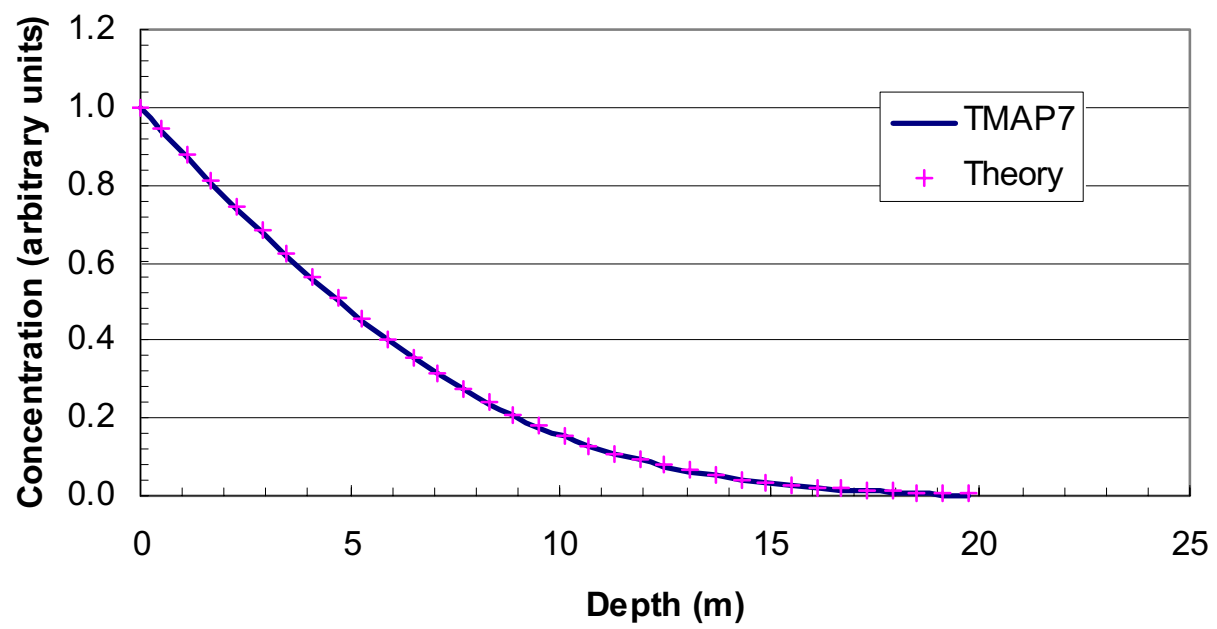

Figure 3. Concentration profile in a semi-infinite slab of $\mathrm{SiC}$ after $25 \mathrm{~s}$ from problem Val-1b.

The third, and final, comparison for this problem was the comparison of the diffusive flux into the slab. The flux into or out of a slab is proportional to the concentration gradient in the $\mathrm{x}$ direction at the slab surface. The solution ${ }^{6}$ is given by 


$$
J=C_{o} \sqrt{\frac{D}{t \pi}} \exp \left(\frac{x}{2 \sqrt{D t}}\right)
$$

The values of Equation (10) were found using Microsoft Excel. They were compared to the values obtained from TMAP7 and can be seen in Table 4 . The variance is never greater than $0.44 \%$.

Table 4. Flux (atom $/ \mathrm{m}^{2} \mathrm{sec}$ ) into semi-infinite slab from a constant source

\begin{tabular}{rrrr}
\hline Time (s) & TMAP7 & Theory & Variance \\
\hline 0 & 1.000000 & 1.000000 & 0.000000 \\
1 & 0.564760 & 0.564190 & 0.001011 \\
2 & 0.399140 & 0.398942 & 0.000496 \\
3 & 0.325840 & 0.325735 & 0.000322 \\
4 & 0.282170 & 0.282095 & 0.000267 \\
5 & 0.252360 & 0.252313 & 0.000185 \\
6 & 0.230370 & 0.230329 & 0.000176 \\
7 & 0.213270 & 0.213244 & 0.000124 \\
8 & 0.199500 & 0.199471 & 0.000145 \\
9 & 0.188080 & 0.188063 & 0.000089 \\
10 & 0.178430 & 0.178412 & 0.000099 \\
11 & 0.170130 & 0.170110 & 0.000120 \\
12 & 0.162880 & 0.162868 & 0.000077 \\
13 & 0.156490 & 0.156478 & 0.000076 \\
14 & 0.150800 & 0.150786 & 0.000093 \\
15 & 0.145680 & 0.145673 & 0.000047 \\
16 & 0.141060 & 0.141047 & 0.000089 \\
17 & 0.136840 & 0.136836 & 0.000029 \\
18 & 0.132990 & 0.132981 & 0.000069 \\
19 & 0.129440 & 0.129434 & 0.000047 \\
20 & 0.126160 & 0.126157 & 0.000027 \\
21 & 0.123120 & 0.123116 & 0.000030 \\
22 & 0.120290 & 0.120286 & 0.000036 \\
23 & 0.117650 & 0.117642 & 0.000071 \\
24 & 0.115170 & 0.115165 & 0.000046 \\
25 & 0.112840 & 0.112838 & 0.000018 \\
26 & 0.110650 & 0.110647 & 0.000030 \\
27 & 0.108580 & 0.108578 & 0.000015 \\
28 & 0.106630 & 0.106622 & 0.000077 \\
29 & 0.104770 & 0.104767 & 0.000025 \\
30 & 0.103010 & 0.103006 & 0.000034 \\
\hline & & & \\
\hline
\end{tabular}

\subsection{Problem 1c: Diffusion in a Partially Preloaded Semi-Infinite Slab (Val-1c)}

This problem models a semi-infinite slab with the first 10 meters preloaded to a uniform concentration. The concentration at the free surface is set to zero for time, $t \geq 0 \mathrm{sec}$, when the pre-loaded inventory is allowed to diffuse out the surface and through the slab. No traps are assumed to be present. Comparisons will be made between TMAP7 and analytical values for 
concentration histories at two locations: one in the initially unloaded region of the slab, at $x=12 \mathrm{~m}$, and one near the surface, $x=0.5 \mathrm{~m}$. A third is made at the end of the preloaded region.

By analogy with Carslaw and Jaeger ${ }^{7}$ the concentration as a function of space and time is

$$
C=\frac{C_{o}}{2}\left[2 \operatorname{erf}\left(\frac{x}{2 \sqrt{D t}}\right)-\operatorname{erf}\left(\frac{x-h}{2 \sqrt{D t}}\right)-\operatorname{erf}\left(\frac{h+x}{2 \sqrt{D t}}\right)\right]
$$

where

$$
\begin{aligned}
& h=\text { thickness of pre-loaded region in the slab }(10 \mathrm{~m}) \\
& C_{o}=\text { concentration of pre-loaded section }\left(1.0 \text { atoms } / \mathrm{m}^{3}\right) \\
& D=\text { diffusion coefficient }\left(1.0 \mathrm{~m}^{2} / \mathrm{sec}\right)
\end{aligned}
$$

Results for the concentration history at $x=12 \mathrm{~m}$ can be seen in Table 5 . The variance for this problem only exceeded $2 \%$ near the peak. Elsewhere it was much less. That could be improved by judicious choice of problem parameters.

Table 5. Concentration history at $x=12$ meters.

\begin{tabular}{cccc}
\hline Time (s) & TMAP7 & Theory & Variance \\
\hline 0 & 0.00000 & 0.00000 & 0.00000 \\
5 & 0.26112 & 0.26340 & -0.00865 \\
10 & 0.32378 & 0.32007 & 0.01159 \\
15 & 0.33563 & 0.32906 & 0.01995 \\
20 & 0.32524 & 0.31839 & 0.02152 \\
25 & 0.30584 & 0.29989 & 0.01983 \\
30 & 0.28373 & 0.27905 & 0.01679 \\
35 & 0.26176 & 0.25832 & 0.01333 \\
40 & 0.24116 & 0.23877 & 0.01000 \\
45 & 0.22234 & 0.22081 & 0.00694 \\
50 & 0.20536 & 0.20450 & 0.00418 \\
55 & 0.19014 & 0.18979 & 0.00183 \\
60 & 0.17650 & 0.17655 & -0.00028 \\
65 & 0.16428 & 0.16463 & -0.00211 \\
70 & 0.15331 & 0.15388 & -0.00371 \\
75 & 0.14345 & 0.14418 & -0.00505 \\
80 & 0.13455 & 0.13540 & -0.00626 \\
85 & 0.12650 & 0.12743 & -0.00729 \\
90 & 0.11920 & 0.12018 & -0.00816 \\
95 & 0.11256 & 0.11357 & -0.00888 \\
100 & 0.10650 & 0.10752 & -0.00951 \\
\hline
\end{tabular}

The next comparison for this model is at $x=0.5 \mathrm{~m}$, the closest node to the surface. The variance for this problem was less than $2 \%$ for all times except when the value approached zero. Reducing the time step and node spacing could decrease the variance at the expense of calculation time required. These values can be seen in Table 6 . 
Table 6. Concentration at $x=0.5$ meters

\begin{tabular}{cccc}
\hline Time (s) & TMAP7 & Theory & Variance \\
\hline 0 & 0.00000 & 0.00000 & 0.00000 \\
5 & 0.12687 & 0.12475 & 0.01699 \\
10 & 0.08249 & 0.08164 & 0.01038 \\
15 & 0.05953 & 0.05893 & 0.01005 \\
20 & 0.04539 & 0.04491 & 0.01062 \\
25 & 0.03603 & 0.03560 & 0.01199 \\
30 & 0.02947 & 0.02907 & 0.01373 \\
35 & 0.02468 & 0.02431 & 0.01527 \\
40 & 0.02104 & 0.02070 & 0.01626 \\
45 & 0.01820 & 0.01790 & 0.01648 \\
50 & 0.01593 & 0.01568 & 0.01597 \\
55 & 0.01409 & 0.01388 & 0.01490 \\
60 & 0.01256 & 0.01240 & 0.01334 \\
65 & 0.01129 & 0.01116 & 0.01144 \\
70 & 0.01021 & 0.01012 & 0.00938 \\
75 & 0.00929 & 0.00923 & 0.00730 \\
80 & 0.00850 & 0.00846 & 0.00524 \\
85 & 0.00782 & 0.00779 & 0.00324 \\
90 & 0.00722 & 0.00721 & 0.00136 \\
95 & 0.00669 & 0.00669 & -0.00038 \\
100 & 0.06224 & 0.00624 & 8.98023 \\
\hline
\end{tabular}

The last comparison is made at $x=\mathrm{h}$. For this case, Equation (11) reduces to

$$
C=\frac{C_{o}}{2}\left[2 \operatorname{erf}\left(\frac{h}{2 \sqrt{D t}}\right)-\operatorname{erf}\left(\frac{h+x}{2 \sqrt{D t}}\right)\right] .
$$

The variance between the values obtained from TMAP7 and Equation (12), has the largest values at times, $t \leq 20 \mathrm{sec}$. For all other times, the variance is less than $0.1 \%$. Again, the variance may be reduced with judicious selection of problem definition parameters. The comparison of TMAP7 calculated values with theory may be seen in Table 7.

Table 7. Concentration at $x=10$ meters

\begin{tabular}{crrr}
\hline Time (s) & TMAP7 & Theory & Variance \\
\hline 0 & 0.50000 & 0.50000 & 0.00000 \\
5 & 0.49862 & 0.49843 & 0.00037 \\
10 & 0.47585 & 0.47465 & 0.00253 \\
15 & 0.43574 & 0.43224 & 0.00810 \\
20 & 0.39160 & 0.38694 & 0.01204 \\
25 & 0.34979 & 0.34504 & 0.01378 \\
30 & 0.31246 & 0.30821 & 0.01379 \\
35 & 0.27993 & 0.27642 & 0.01270 \\
40 & 0.25185 & 0.24912 & 0.01097 \\
45 & 0.22768 & 0.22567 & 0.00891 \\
50 & 0.20683 & 0.20544 & 0.00675
\end{tabular}




\begin{tabular}{cccr}
\hline Time (s) & TMAP7 & Theory & Variance \\
\hline 55 & 0.18877 & 0.18791 & 0.00458 \\
60 & 0.17306 & 0.17263 & 0.00249 \\
65 & 0.15933 & 0.15925 & 0.00052 \\
70 & 0.14727 & 0.14746 & -0.00126 \\
75 & 0.13662 & 0.13702 & -0.00289 \\
80 & 0.12716 & 0.12773 & -0.00442 \\
85 & 0.11874 & 0.11942 & -0.00573 \\
90 & 0.11119 & 0.11196 & -0.00687 \\
95 & 0.10441 & 0.10524 & -0.00789 \\
100 & 0.09828 & 0.09915 & -0.00879 \\
\hline
\end{tabular}

\subsection{Problem 1d: Permeation Problem with Trapping (Val-1da, Val-1db, Val-1dc)}

The following three models simulate diffusion through a slab in which traps are operational. The three trapping regimes demonstrated are an effective diffusivity trap, a strong trap, and a set of three traps in the effective diffusivity range with different trap strengths. The diffusion boundary conditions for this set of problems are fixed-concentration or sconc, with one surface kept at a constant non-zero concentration and the other set at zero concentration. Initially, the slab is empty. Validation criteria for these problems will be the comparison of the flux and breakthrough times for each of the models with idealizations. The breakthrough time of the flux may have one of two limiting values, which depend on whether the trapping is in the effective diffusivity or strong-trapping regime. A trapping parameter ${ }^{8}$ is defined by

$$
\varsigma=\frac{\lambda^{2} v}{D_{o} \rho} \exp \left(\frac{E_{d}-\varepsilon}{k t}\right)+\frac{c}{\rho}
$$

where

$$
\begin{aligned}
& \left.\lambda=\text { lattice parameter (assume } 3.162 \times 10^{-8} \mathrm{~m}\right) \\
& v=\text { Debye frequency }\left(1 \times 10^{13} \mathrm{~s}^{-1}\right) \\
& \rho=\text { trapping site fraction }(0.1) \\
& D_{0}=\text { diffusivity pre-exponential }\left(1 \mathrm{~m}^{2} / \mathrm{sec}\right) \\
& \mathrm{E}_{\mathrm{d}}=\text { diffusion activation energy } \\
& \varepsilon=\text { trap energy } \\
& \mathrm{k}=\text { Boltzmann's constant } \\
& \mathrm{T}=\text { temperature }(1000 \mathrm{~K}) \\
& \mathrm{c}=\text { surface dissolved gas atom fraction. }(0.0001)
\end{aligned}
$$

The determining value for which regime is dominant is the relation of $\varsigma$ to $c / \rho$. If $\varsigma>>c / \rho$, then the effective diffusivity regime applies, and the flux transient is identical to the standard diffusion transient, but with the diffusivity replaced by an effective diffusivity, 


$$
D_{e f f}=\frac{D}{1+\sum_{i} \frac{1}{\varsigma_{i}}}
$$

In this limit, the breakthrough time, defined as the intersection of the steepest tangent of the diffusion transient with the time axis, will be

$$
\tau_{b_{e}}=\frac{\boldsymbol{l}^{2}}{2 \pi^{2} \boldsymbol{D}_{\text {eff }}}
$$

where

$$
\begin{aligned}
& 1=\text { thickness of slab }(1 \mathrm{~m}) \\
& D=\text { diffusivity of gas }\left(1 \mathrm{~m}^{2} / \mathrm{s}\right) .
\end{aligned}
$$

The permeation transient is then given by

$$
\boldsymbol{J}_{p}=\frac{\boldsymbol{c}_{\boldsymbol{o}} \boldsymbol{D}}{\boldsymbol{l}}\left[1+2 \sum_{\boldsymbol{m}=1}^{\infty}(-1)^{m} \exp \left(-\boldsymbol{m}^{2} \frac{\boldsymbol{t}}{2 \tau_{b_{e}}}\right)\right]
$$

where $\tau_{\text {be }}$ is as defined in Equation (15).

\subsubsection{Effective Diffusivity Trap (Val-1da)}

The first example is the case where a single trap is in the effective diffusivity limit. The ratio $\varepsilon / k$ (see Equation (13)) was taken as 100 , to give a value of $\zeta=101 \mathrm{c} / \rho$. TMAP7's breakthrough time was found numerically by using a three-point differentiation method given by Fogler ${ }^{9}$ to find the steepest slope.

$$
\left(\frac{d C_{A}}{d t}\right)_{t_{i}}=\frac{1}{2 \Delta t}\left[C_{A(i+1)}-C_{A(i-1)}\right] \approx m
$$

Then, the point where the slope was the steepest was used with the slope at that point to find the intersection with the time axis. This was computed to be 0.5999 seconds. The analytical breakthrough time was calculated to be 0.611 seconds. The variance between theoretical values of the permeation flux and those calculated by TMAP7 using this model is less than $2 \%$, for most times, as shown in Figure 4. Variance would be less for, say, $\zeta=1000 \mathrm{c} / \rho$. The permeation curve where no trapping is present is also shown in Figure 4 to illustrate the retarding of the permeation curve by a trap. 


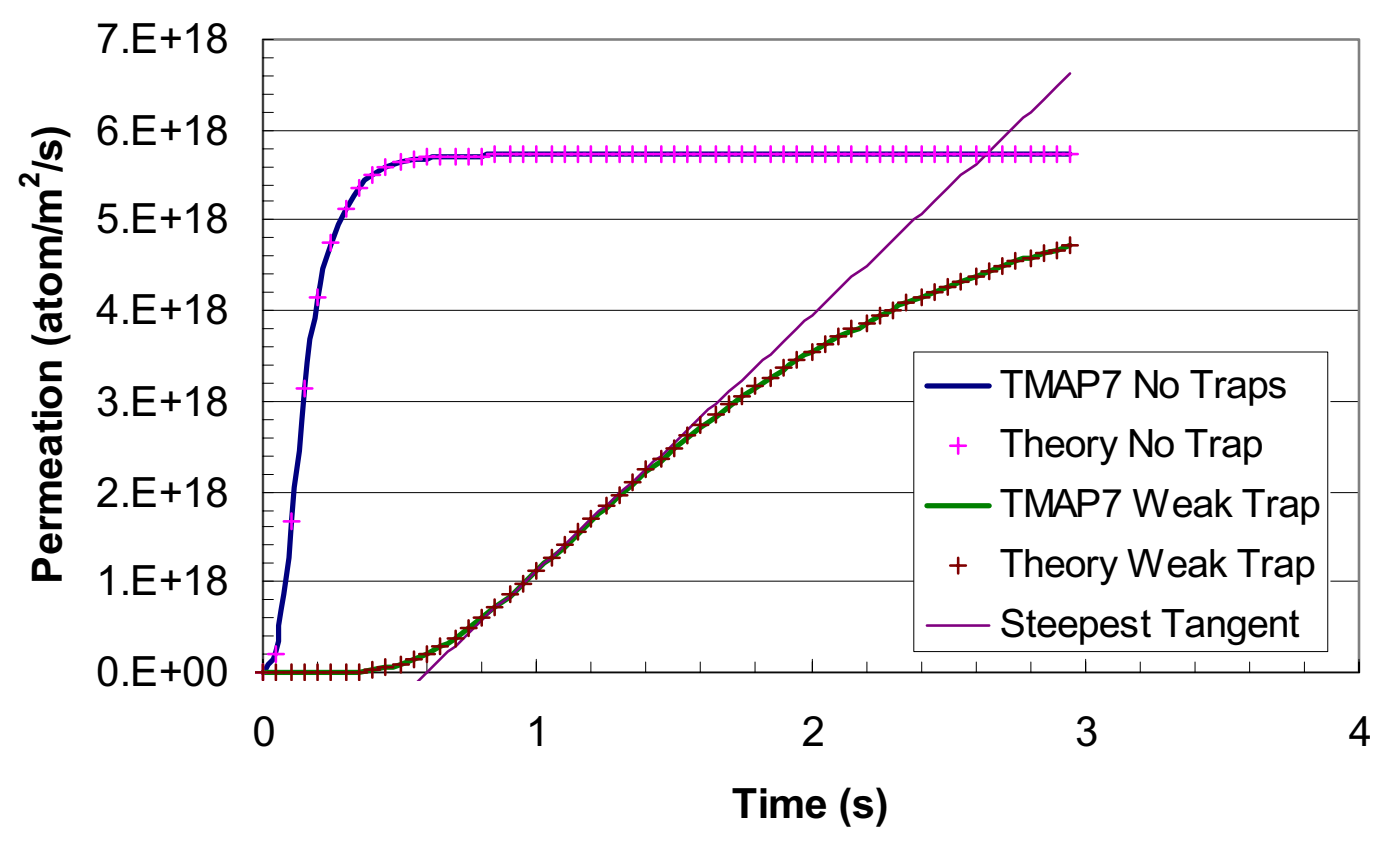

Figure 4. Effective-diffusivity, single trap (Val-1da, Val-1da1).

\subsubsection{Strong Trap (Val-1db)}

In the second model, $\varsigma<<\mathrm{c} / \rho$, is applied to obtain a strong trapping regime. In this regime, no permeation occurs until essentially all the traps have been filled. Then the permeation rapidly turns on to its steady state value. This is due to the relatively low release of trapped atoms. The breakthrough time is given by

$$
\tau_{b_{d}}=\frac{l^{2} \rho}{2 c_{o} D}
$$

where $c_{o}, \rho, l$, and $D$ are defined as in the first model. The value of $\varepsilon / k$ is taken to be $15,000 \mathrm{~K}$, to give $\zeta=c / \rho$. The only difference in the input file between the first and second models is this parameter and a larger time step. The breakthrough time in the strong trapping regime was taken as the first time that the permeation was at its steady state value. This occurred at 511 seconds. The estimated breakthrough time from Eq. (18) is 500 seconds. The permeation curve for this model can be seen in Figure 5. 


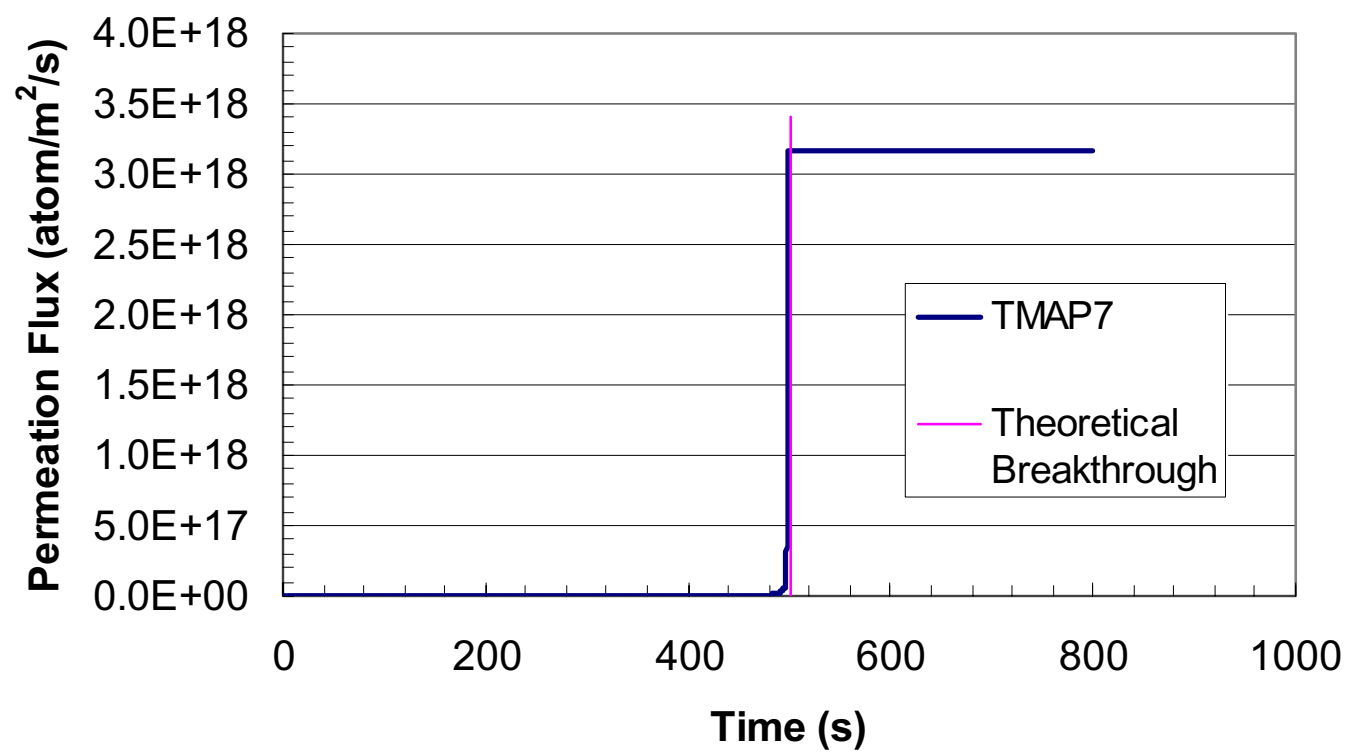

Figure 5. Permeation for strong-trapping regime (Val-1db)

\subsubsection{Multiple Trap (Val-1dc)}

The last problem modeled in this section demonstrates the effects of multiple traps. This feature is new to TMAP7. To illustrate TMAP7's capabilities to allow for multiple traps, three traps that are relatively weak are assumed to be active in a slab. The parameters of the first trap are the same as the trap in the effective diffusivity limit, first model. The second and third traps vary by having trap concentrations of 0.15 and 0.20 atom fractions and the values of $\varepsilon / k$ chosen to be $500 \mathrm{~K}$ and $800 \mathrm{~K}$, respectively. These values give the following values for $\mathrm{s}$ :

Trap 1: $181.97 \mathrm{c} / \rho$

Trap 2: $122.31 \mathrm{c} / \rho$

Trap 3: $90.87 c / \rho$.

The effective diffusivity was calculated from Equation (14), $D_{\text {eff }}=0.01242 \mathrm{~m}^{2} / \mathrm{sec}$, and the breakthrough time was calculated from Equation (15) to be $4.08 \mathrm{sec}$. TMAP7's calculated breakthrough time was $4.00 \mathrm{sec}$. The permeation curves that were calculated using Equation (16) are compared with TMAP7 results in Figure 6. The graphs for the theoretical flux and the calculated flux are nearly identical. 


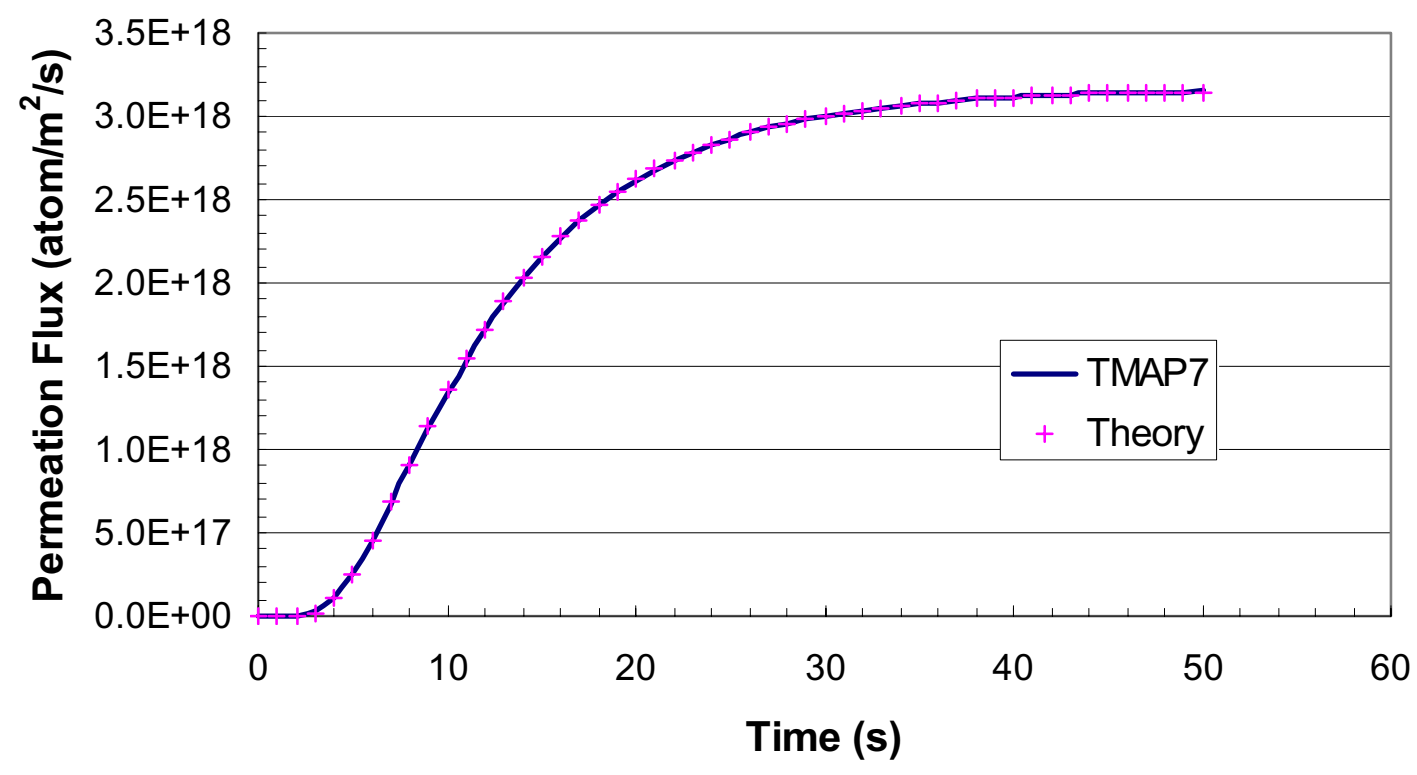

Figure 6. Permeation curve for slab with multiple traps (Val-1dc).

\subsection{Problem 1e: Diffusion with Composite Material Layers (Val-1e)}

A composite structure of $\mathrm{PyC}$ and $\mathrm{SiC}$ is modeled with a constant concentration boundary condition on the free surface of the $\mathrm{PyC}$ and a zero-concentration boundary on the free surface of the $\mathrm{SiC}$. The concentration profile in steady state is to be analyzed. The steady-state solution for the PyC is given in Eq. (19),

$$
C=C_{o}\left[1+\frac{x}{a}\left(\frac{D_{P y C} l}{l D_{P y C}+a D_{S i C}}-1\right)\right]
$$

while the concentration profile for the $\mathrm{SiC}$ is given by

$$
C=C_{o}\left(\frac{a+l-x}{l}\right)\left(\frac{D_{P y C} l}{l D_{P y C}+a D_{S i C}}\right)
$$

where

$$
\begin{aligned}
& a=\text { thickness of the PyC layer }(33 \mu \mathrm{m}) \\
& l=\text { thickness of the } \mathrm{SiC} \text { layer }(66 \mu \mathrm{m}) \\
& \mathrm{C}_{\mathrm{o}}=\text { the concentration at the surface }\left(3.0537 \times 10^{25} \text { atoms } / \mathrm{m}^{3}\right) \\
& S_{a}=\text { Solubility of both species was taken as } 1.0 \text { (units arbitrary) }
\end{aligned}
$$

The values for the diffusivity were taken as constants, $D_{P y C}=1.274 \times 10^{-7} \mathrm{~m}^{2 /} \mathrm{sec}$ and $D_{S i C}=2.622 \times 10^{-11} \mathrm{~m}^{2} / \mathrm{sec}$. The variance for this problem does not exceed $0.004 \%$. The comparison of Eqs. (19) and (20) with TMAP7's values can be seen in Table 8. 
Table 8. Steady-State Concentration Profile in Composite Slab

\begin{tabular}{rrrr}
\hline Depth $(\mathbf{m})$ & TMAP7 & Theory & Variance \\
\hline $0.00 \mathrm{E}+00$ & $3.0537 \mathrm{E}+25$ & $3.0537 \mathrm{E}+25$ & $0.00 \mathrm{E}+00$ \\
$1.50 \mathrm{E}-06$ & $3.0537 \mathrm{E}+25$ & $3.0537 \mathrm{E}+25$ & $-4.68 \mathrm{E}-06$ \\
$5.50 \mathrm{E}-06$ & $3.0536 \mathrm{E}+25$ & $3.0536 \mathrm{E}+25$ & $1.56 \mathrm{E}-05$ \\
$1.05 \mathrm{E}-05$ & $3.0536 \mathrm{E}+25$ & $3.0536 \mathrm{E}+25$ & $8.27 \mathrm{E}-09$ \\
$1.55 \mathrm{E}-05$ & $3.0536 \mathrm{E}+25$ & $3.0536 \mathrm{E}+25$ & $-1.56 \mathrm{E}-05$ \\
$2.05 \mathrm{E}-05$ & $3.0535 \mathrm{E}+25$ & $3.0535 \mathrm{E}+25$ & $1.58 \mathrm{E}-06$ \\
$2.55 \mathrm{E}-05$ & $3.0535 \mathrm{E}+25$ & $3.0535 \mathrm{E}+25$ & $-1.40 \mathrm{E}-05$ \\
$3.05 \mathrm{E}-05$ & $3.0534 \mathrm{E}+25$ & $3.0534 \mathrm{E}+25$ & $3.14 \mathrm{E}-06$ \\
$3.30 \mathrm{E}-05$ & $3.0534 \mathrm{E}+25$ & $3.0534 \mathrm{E}+25$ & $-4.65 \mathrm{E}-06$ \\
$3.30 \mathrm{E}-05$ & $3.0534 \mathrm{E}+25$ & $3.0534 \mathrm{E}+25$ & $-4.65 \mathrm{E}-06$ \\
$3.83 \mathrm{E}-05$ & $2.8105 \mathrm{E}+25$ & $2.8105 \mathrm{E}+25$ & $1.01 \mathrm{E}-06$ \\
$4.88 \mathrm{E}-05$ & $2.3247 \mathrm{E}+25$ & $2.3247 \mathrm{E}+25$ & $1.59 \mathrm{E}-05$ \\
$5.93 \mathrm{E}-05$ & $1.8390 \mathrm{E}+25$ & $1.8390 \mathrm{E}+25$ & $-1.58 \mathrm{E}-05$ \\
$6.98 \mathrm{E}-05$ & $1.3532 \mathrm{E}+25$ & $1.3532 \mathrm{E}+25$ & $3.74 \mathrm{E}-06$ \\
$8.03 \mathrm{E}-05$ & $8.6744 \mathrm{E}+24$ & $8.6744 \mathrm{E}+24$ & $-9.85 \mathrm{E}-07$ \\
$9.08 \mathrm{E}-05$ & $3.8167 \mathrm{E}+24$ & $3.8167 \mathrm{E}+24$ & $8.45 \mathrm{E}-06$ \\
$9.75 \mathrm{E}-05$ & $6.9395 \mathrm{E}+23$ & $6.9395 \mathrm{E}+23$ & $1.90 \mathrm{E}-06$ \\
$9.90 \mathrm{E}-05$ & $0.0000 \mathrm{E}+00$ & $0.0000 \mathrm{E}+00$ & $0.00 \mathrm{E}+00$ \\
\hline
\end{tabular}

Demonstration of transient agreement with theory may also be shown by examining the concentration history at a point $7.5 \mu \mathrm{m}$ into the $\mathrm{SiC}$ layer as a function of time given that, initially, both $\mathrm{PyC}$ and $\mathrm{SiC}$ were empty of gas. The transient solution for concentration in the $\mathrm{SiC}$ side of the composite slab is

$$
C=C_{o}\left\{\frac{D_{P y C}(l-x)}{l D_{P y C}+a D_{S i C}}-2 \sum_{n=1}^{\infty} \frac{\sin \left(a \lambda_{n}\right) \sin \left(k l \lambda_{n}\right) \sin \left[k(l-x) \lambda_{n}\right]}{\lambda_{n}\left[a \sin \left(k l \lambda_{n}\right)+l \sin ^{2}\left(a \lambda_{n}\right)\right]} \exp \left(-D_{P y C} \lambda_{n}^{2} t\right)\right\}
$$

where

$$
\begin{aligned}
a & =\text { thickness of } \operatorname{PyC}(33 \mu \mathrm{m}) \\
l & =\text { Thickness of } \operatorname{SiC}(66 \mu \mathrm{m}) \\
k & =\sqrt{\frac{D_{P y C}}{D_{S i C}}}=69.7036
\end{aligned}
$$

and the $\lambda_{n}$ are the roots of

$$
\tan (\lambda a)+k \tan (k \lambda l)=0
$$

Figure 7 shows the graphical comparison, and Table 9 lists discreet values and variance. The series in Eq. (21) was evaluated with 19 terms such that the contribution of the last term was less than 1.0E-11 for all times. The fit improves with finer spatial mesh. 


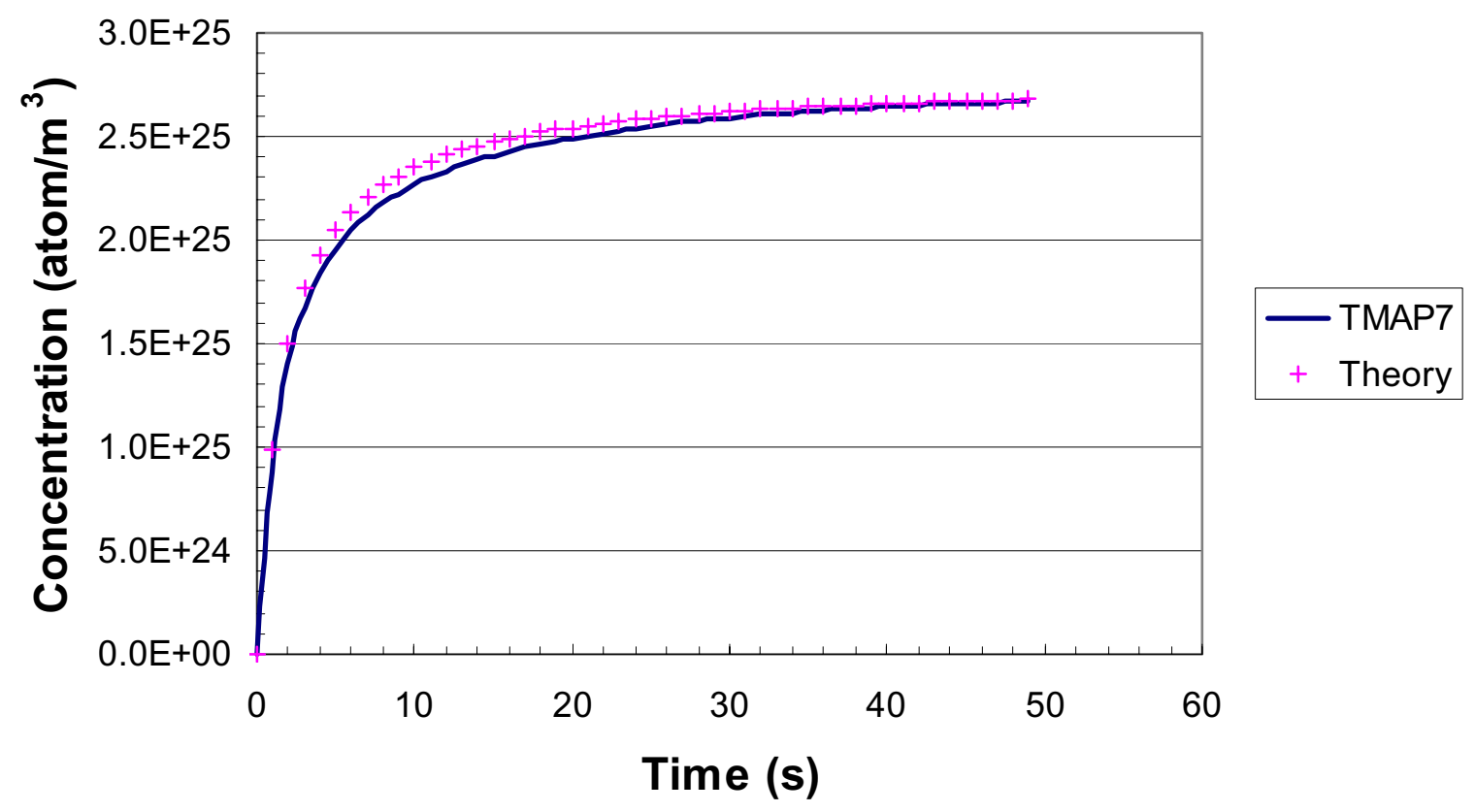

Figure 7. Comparison of TMAP7 with theoretical solution for transient concentration at a location $7.5 \mu \mathrm{m}$ deep in the $\mathrm{SiC}$ layer of a composite slab of PyC and $\mathrm{SiC}$ (Val-1e).

Table 9. Variance for transient solution in composite slab.

\begin{tabular}{clll}
\hline Time (s) & TMAP7 & Theory & Variance \\
\hline 0 & $0.00 \mathrm{E}+00$ & $0.00 \mathrm{E}+00$ & 0.000000 \\
1 & $8.81 \mathrm{E}+24$ & $9.92 \mathrm{E}+24$ & 0.111677 \\
2 & $1.40 \mathrm{E}+25$ & $1.50 \mathrm{E}+25$ & 0.067949 \\
3 & $1.67 \mathrm{E}+25$ & $1.76 \mathrm{E}+25$ & 0.055549 \\
4 & $1.84 \mathrm{E}+25$ & $1.93 \mathrm{E}+25$ & 0.049649 \\
5 & $1.96 \mathrm{E}+25$ & $2.05 \mathrm{E}+25$ & 0.045956 \\
6 & $2.05 \mathrm{E}+25$ & $2.14 \mathrm{E}+25$ & 0.043165 \\
7 & $2.12 \mathrm{E}+25$ & $2.21 \mathrm{E}+25$ & 0.040838 \\
8 & $2.18 \mathrm{E}+25$ & $2.26 \mathrm{E}+25$ & 0.038742 \\
9 & $2.23 \mathrm{E}+25$ & $2.31 \mathrm{E}+25$ & 0.036767 \\
10 & $2.27 \mathrm{E}+25$ & $2.35 \mathrm{E}+25$ & 0.034865 \\
11 & $2.30 \mathrm{E}+25$ & $2.38 \mathrm{E}+25$ & 0.032995 \\
12 & $2.33 \mathrm{E}+25$ & $2.41 \mathrm{E}+25$ & 0.031260 \\
13 & $2.36 \mathrm{E}+25$ & $2.43 \mathrm{E}+25$ & 0.029555 \\
14 & $2.39 \mathrm{E}+25$ & $2.45 \mathrm{E}+25$ & 0.027897 \\
15 & $2.41 \mathrm{E}+25$ & $2.47 \mathrm{E}+25$ & 0.026365 \\
16 & $2.43 \mathrm{E}+25$ & $2.49 \mathrm{E}+25$ & 0.024870 \\
17 & $2.45 \mathrm{E}+25$ & $2.51 \mathrm{E}+25$ & 0.023453 \\
18 & $2.46 \mathrm{E}+25$ & $2.52 \mathrm{E}+25$ & 0.022090 \\
19 & $2.48 \mathrm{E}+25$ & $2.53 \mathrm{E}+25$ & 0.020817 \\
20 & $2.49 \mathrm{E}+25$ & $2.54 \mathrm{E}+25$ & 0.019579 \\
21 & $2.51 \mathrm{E}+25$ & $2.55 \mathrm{E}+25$ & 0.018465 \\
22 & $2.52 \mathrm{E}+25$ & $2.56 \mathrm{E}+25$ & 0.017358
\end{tabular}




\begin{tabular}{cllc}
\hline Time (s) & TMAP7 & Theory & Variance \\
\hline 23 & $2.53 \mathrm{E}+25$ & $2.57 \mathrm{E}+25$ & 0.016332 \\
24 & $2.54 \mathrm{E}+25$ & $2.58 \mathrm{E}+25$ & 0.015376 \\
25 & $2.55 \mathrm{E}+25$ & $2.59 \mathrm{E}+25$ & 0.014475 \\
26 & $2.56 \mathrm{E}+25$ & $2.59 \mathrm{E}+25$ & 0.013650 \\
27 & $2.57 \mathrm{E}+25$ & $2.60 \mathrm{E}+25$ & 0.012839 \\
28 & $2.58 \mathrm{E}+25$ & $2.61 \mathrm{E}+25$ & 0.012059 \\
29 & $2.58 \mathrm{E}+25$ & $2.61 \mathrm{E}+25$ & 0.011361 \\
30 & $2.59 \mathrm{E}+25$ & $2.62 \mathrm{E}+25$ & 0.010718 \\
31 & $2.60 \mathrm{E}+25$ & $2.62 \mathrm{E}+25$ & 0.010063 \\
32 & $2.60 \mathrm{E}+25$ & $2.63 \mathrm{E}+25$ & 0.009481 \\
33 & $2.61 \mathrm{E}+25$ & $2.63 \mathrm{E}+25$ & 0.008906 \\
34 & $2.62 \mathrm{E}+25$ & $2.64 \mathrm{E}+25$ & 0.008418 \\
35 & $2.62 \mathrm{E}+25$ & $2.64 \mathrm{E}+25$ & 0.007913 \\
36 & $2.63 \mathrm{E}+25$ & $2.65 \mathrm{E}+25$ & 0.007433 \\
37 & $2.63 \mathrm{E}+25$ & $2.65 \mathrm{E}+25$ & 0.007022 \\
38 & $2.63 \mathrm{E}+25$ & $2.65 \mathrm{E}+25$ & 0.006609 \\
39 & $2.64 \mathrm{E}+25$ & $2.66 \mathrm{E}+25$ & 0.006237 \\
40 & $2.64 \mathrm{E}+25$ & $2.66 \mathrm{E}+25$ & 0.005872 \\
\hline
\end{tabular}

\subsection{Problem 1f: Heat Sink/Source Problem}

Four heat transfer models were set up to validate the heat transfer capabilities of the TMAP7 code. The four problems solved include (a) heat conduction with generation; (b) conduction modeled as mass transfer, (c) transient conduction and steady state values in a composite structure, and (d) heating of a semi-infinite slab by convection.

\subsubsection{Heat conduction with generation (Val-1 fa)}

To model the first problem, the thermal boundary conditions were set so one surface was adiabatic, while the other was kept at constant temperature. The heat generation in the slab was assumed to be constant throughout. Incropera and DeWitt ${ }^{10}$ give the analytical solution for the steady state temperature of this model as

$$
T=T_{s}+\frac{Q L^{2}}{2 k}\left(1-\frac{x^{2}}{L^{2}}\right)
$$

where

$$
\begin{aligned}
& Q=\text { internal heat generation rate }\left(10,000 \mathrm{~W} / \mathrm{m}^{3}\right) \\
& L=\text { thickness of slab }(1.6 \mathrm{~m}) \\
& k=\text { thermal conductivity }(10 \mathrm{~W} / \mathrm{m} \mathrm{K}) \\
& T_{s}=\text { surface temperature }(300 \mathrm{~K})
\end{aligned}
$$

A value for thermal mass, the product of material mass density and specific heat, must be added for TMAP7 thermal calculations. In this problem, $\rho c_{p}=1 \mathrm{~J} / \mathrm{m}^{3} \mathrm{~K}$ was assumed. Initially, 16 spatial segments were assumed. The variance for this problem was less than $0.2 \%$ for distances less than $1.35 \mathrm{~m}$, but it increased as the distance from the adiabatic surface was 
increased. To show that this can be reduced with a decrease in the distance between nodes, an additional calculation was performed with 48 spatial segments. The variance was reduced by a factor of approximately 10 . The comparison of Eq. (23) with TMAP7 values can be seen in Table 10.

Table 10. Heat Conduction with Generation

\begin{tabular}{|c|c|c|c|c|c|}
\hline Position (m) & Theory & $\mathbf{1 6}$ Segs & Variance & $\mathbf{4 8 ~ S e g s}$ & Variance \\
0.00 & 1580.00 & 1580.00 & 0.00000 & 1580.00 & 0.00000 \\
0.05 & 1578.75 & 1580.00 & 0.00079 & 1578.90 & 0.00010 \\
0.15 & 1568.75 & 1570.00 & 0.00080 & 1568.90 & 0.00010 \\
0.25 & 1548.75 & 1550.00 & 0.00081 & 1548.90 & 0.00010 \\
0.35 & 1518.75 & 1520.00 & 0.00082 & 1518.90 & 0.00010 \\
0.45 & 1478.75 & 1480.00 & 0.00085 & 1478.90 & 0.00010 \\
0.55 & 1428.75 & 1430.00 & 0.00087 & 1428.90 & 0.00010 \\
0.65 & 1368.75 & 1370.00 & 0.00091 & 1368.90 & 0.00011 \\
0.75 & 1298.75 & 1300.00 & 0.00096 & 1298.90 & 0.00012 \\
0.85 & 1218.75 & 1220.00 & 0.00103 & 1218.90 & 0.00012 \\
0.95 & 1128.75 & 1130.00 & 0.00111 & 1128.90 & 0.00013 \\
1.05 & 1028.75 & 1030.00 & 0.00122 & 1028.90 & 0.00015 \\
1.15 & 918.75 & 920.00 & 0.00136 & 918.88 & 0.00014 \\
1.25 & 798.75 & 800.00 & 0.00156 & 798.88 & 0.00016 \\
1.35 & 668.75 & 670.00 & 0.00187 & 668.88 & 0.00019 \\
1.45 & 528.75 & 530.00 & 0.00236 & 528.88 & 0.00025 \\
1.55 & 378.75 & 380.00 & 0.00330 & 378.89 & 0.00037 \\
1.60 & 300.00 & 300.00 & 0.00000 & 300.00 & 0.00000 \\
\hline
\end{tabular}

\subsubsection{Thermal Diffusion Transient (Val-1fb)}

The second problem validates the thermal diffusion capability in a slab. The temperature of the left side of the thermal segment was held constant at $400 \mathrm{~K}$ while the right side was held at a constant $300 \mathrm{~K}$. The initial temperature in the slab was $300 \mathrm{~K}$. For this example, the thickness, $\mathrm{L}$, was $4 \mathrm{~m}$ and the heat production rate was $Q=0$. Mass diffusion was ignored by setting the mobile species concentration to zero and using non-flow boundaries. The analytical solution is given by

$$
T(x, t)=T_{o}+\left(T_{1}-T_{o}\right)\left[1-\frac{x}{L}-\frac{2}{L} \sum_{m=0}^{\infty} \frac{1}{\lambda_{m}} \sin \left(\lambda_{m} x\right) \exp \left(-\alpha \lambda_{m}^{2} t\right)\right]
$$

where

$$
\lambda_{m}=m \frac{\pi}{L}
$$

and thermal diffusivity is

$$
\alpha=\frac{k}{C_{p} \rho}
$$

For the problem analyzed, 


$$
\begin{aligned}
& \alpha=1.0 \mathrm{~m}^{2} / \mathrm{s}, \\
& \mathrm{T}_{\mathrm{o}}=300 \mathrm{~K}, \text { and } \\
& \mathrm{T}_{1}=400 \mathrm{~K} .
\end{aligned}
$$

The values for Eq. (24) were found using MS Excel. The last term in the summation taken contributed less than $1 \times 10^{-13}$ of the theoretical value. The agreement between TMAP7 and Eq. (24) is excellent, with the variance less than $1 \%$ for each case tested, and usually much less. The comparison between the values can be seen in Figure 8 for temperature profiles through the slab at $0.1,0.5,1.0$, and 5.0 seconds.

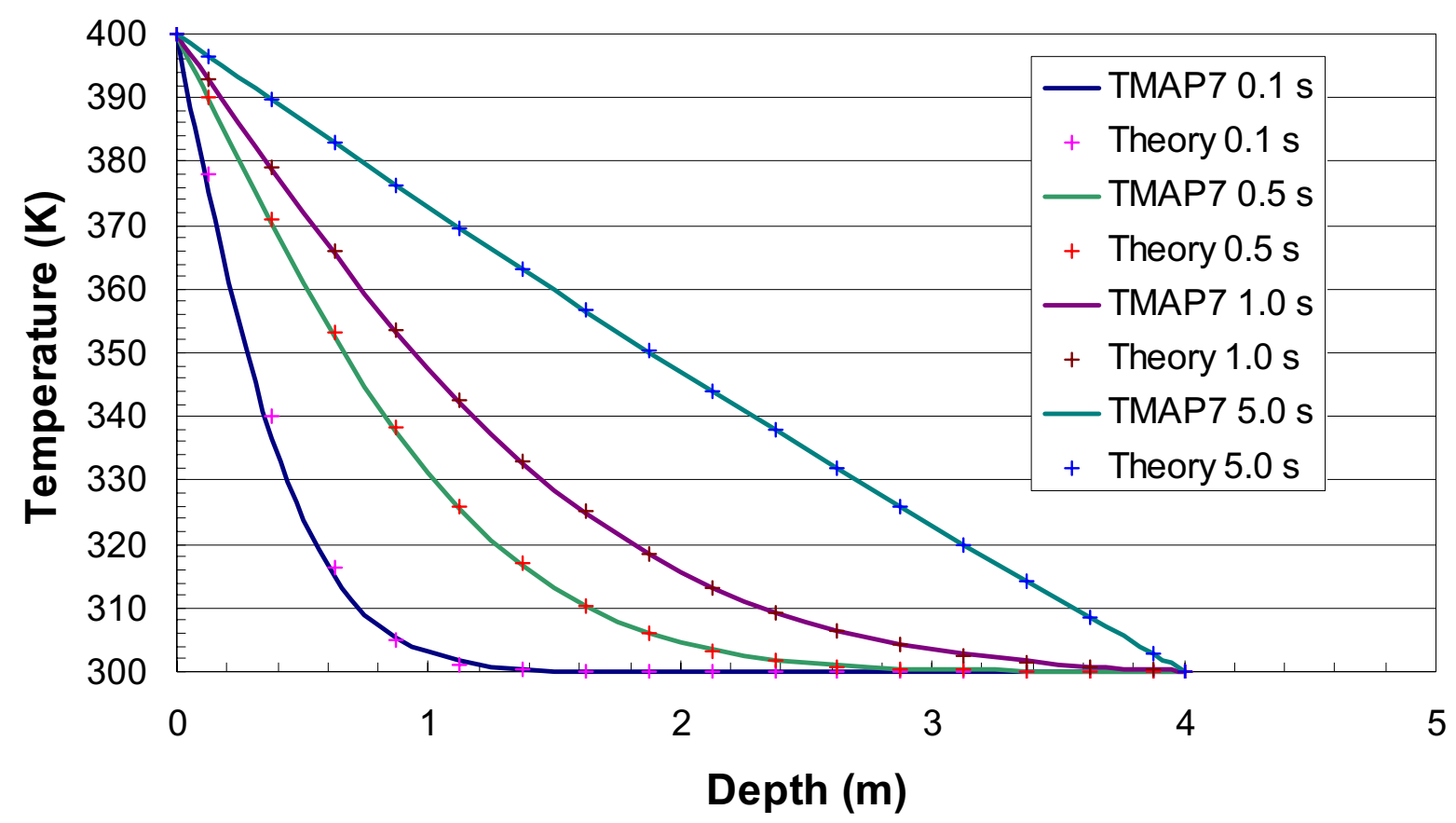

Figure 8. Transient temperature distribution for various times in a slab (Val-1fb).

\subsubsection{Conduction in Composite Structure with Constant Surface Temperatures (Val-1fc)}

The third heat transfer problem studied was heat transfer through a composite slab with constant surface temperatures. The composite was a $40-\mathrm{cm}$ thick layer of $\mathrm{Cu}$ followed by a 40 -cm layer of Fe. The temperature of both layers was initially $0 \mathrm{~K}$, but at time $\underline{\mathrm{t}}=0$, the outside face of the copper was held at $600 \mathrm{~K}$ while the outside face of the Fe was maintained at $0 \mathrm{~K}$. This problem was modeled using the heat transfer capability of TMAP7. The computational answers obtained by TMAP7 for both the transient and steady state solutions were compared to values obtained from ABAQUS. ${ }^{11}$ The ABAQUS code was setup and run by R. G. Ambrosek. ABAQUS is a heat transfer program that has been validated for both transient and steady state solutions. The transient solution was compared at a constant time and constant distance. The constant time comparison between ABAQUS and TMAP7 was made at time, $t=150 \mathrm{sec}$. The 
variance in this comparison grows with increasing distance. This may be due to the time interval on both programs being larger than needed, or round-off error from the printed values. These values can be seen in Table 11.

Table 11. Temperature distribution in composite structure at $t=150$ seconds.

\begin{tabular}{rrrr}
\hline Depth $(\mathbf{m})$ & ABAQUS & TMAP7 & Variance \\
\hline 0.00 & 600.000 & 600.000 & 0.00000 \\
0.01 & 574.400 & 574.370 & -0.00005 \\
0.03 & 523.600 & 523.400 & -0.00038 \\
0.05 & 473.600 & 473.300 & -0.00063 \\
0.07 & 425.100 & 424.630 & -0.00111 \\
0.09 & 378.400 & 377.880 & -0.00137 \\
0.11 & 334.100 & 333.500 & -0.00180 \\
0.13 & 292.500 & 291.850 & -0.00222 \\
0.15 & 253.900 & 253.200 & -0.00276 \\
0.17 & 218.500 & 217.770 & -0.00334 \\
0.19 & 186.400 & 185.670 & -0.00392 \\
0.21 & 157.700 & 156.930 & -0.00488 \\
0.23 & 132.200 & 131.510 & -0.00522 \\
0.25 & 110.000 & 109.320 & -0.00618 \\
0.27 & 90.790 & 90.199 & -0.00651 \\
0.29 & 74.480 & 73.975 & -0.00678 \\
0.31 & 60.860 & 60.439 & -0.00692 \\
0.33 & 49.690 & 49.374 & -0.00636 \\
0.35 & 40.770 & 40.564 & -0.00505 \\
\hline
\end{tabular}

The values were also compared at $x=0.09 \mathrm{~m}$, at 5 second intervals from time $t=0$ to $150 \mathrm{sec}$. The variance is initially large, but reduces as the time increases. The initially large variance may be due to the same factors of spatial resolution and time step size mentioned earlier. These results can be seen in Table 12.

The steady-state solution for this problem was compared to the analytical solution in addition to the ABAQUS answer. To solve for the steady state solution for this problem, the heat flux is given by

$$
q^{\prime \prime}=\frac{T_{S_{A}}-T_{S_{B}}}{\frac{L_{A}}{k_{A}}+\frac{L_{B}}{k_{B}}}
$$

where

$$
\begin{aligned}
& T_{s i}=\text { Temperature of surface } \mathrm{i}, \text { left }(\mathrm{A}) \text { and right (B), } \\
& L_{i} \quad=\text { Length of segment } \mathrm{i} \\
& k_{i} \quad=\text { thermal conductivity of segment } \mathrm{i} .
\end{aligned}
$$


Table 12. Temperature history in composite structure at $x=0.09$ meters

\begin{tabular}{crrr}
\hline Time (s) & TMAP7 & ABAQUS & Variance \\
\hline 0 & 0.00 & 0.00 & 0.00000 \\
5 & 6.50 & 10.11 & 0.35692 \\
10 & 37.03 & 43.47 & 0.14817 \\
15 & 75.11 & 81.42 & 0.07745 \\
20 & 110.42 & 115.90 & 0.04728 \\
25 & 140.99 & 145.60 & 0.03166 \\
30 & 167.17 & 171.10 & 0.02297 \\
35 & 189.68 & 193.10 & 0.01771 \\
40 & 209.20 & 212.10 & 0.01367 \\
45 & 226.29 & 228.90 & 0.01140 \\
50 & 241.39 & 243.70 & 0.00948 \\
55 & 254.84 & 256.90 & 0.00802 \\
60 & 266.91 & 268.70 & 0.00666 \\
65 & 277.82 & 279.50 & 0.00601 \\
70 & 287.74 & 289.36 & 0.00560 \\
75 & 296.81 & 298.30 & 0.00499 \\
80 & 305.14 & 306.40 & 0.00411 \\
85 & 312.83 & 314.00 & 0.00373 \\
90 & 319.95 & 321.00 & 0.00327 \\
95 & 326.57 & 327.60 & 0.00314 \\
100 & 332.74 & 333.70 & 0.00288 \\
105 & 338.52 & 339.40 & 0.00259 \\
110 & 343.94 & 344.80 & 0.00249 \\
115 & 349.04 & 349.80 & 0.00217 \\
120 & 353.85 & 354.60 & 0.00212 \\
125 & 358.39 & 359.10 & 0.00198 \\
130 & 362.70 & 363.40 & 0.00193 \\
135 & 366.78 & 367.40 & 0.00169 \\
140 & 370.66 & 371.30 & 0.00172 \\
145 & 374.36 & 374.90 & 0.00144 \\
150 & 377.88 & 378.40 & 0.00137 \\
\hline & & &
\end{tabular}

For the solution to be at steady state, the flux in and out of any section of the slab must be equal. The temperature at the interface can be found by setting the flux through $A$ equal to the flux through $B$.

$$
\frac{T_{S_{A}}-T_{I}}{\frac{L_{A}}{k_{A}}}=\frac{T_{I}-T_{S_{B}}}{\frac{L_{B}}{k_{B}}}
$$

where

$$
\begin{aligned}
& T_{I}=\text { temperature of interface, } \\
& k_{A}=401 \mathrm{~W} / \mathrm{m} \mathrm{K},
\end{aligned}
$$




$$
\begin{aligned}
& k_{B}=80.2 \mathrm{~W} / \mathrm{m} \mathrm{K}, \\
& L_{A}=L_{B}=0.4 \text { meters, } \\
& T_{S_{A}}=600 \mathrm{~K} \\
& T_{S_{B}}=0 \mathrm{~K}
\end{aligned}
$$

From Eq. (28), the interface temperature is found to be $T_{I}=500 \mathrm{~K}$. The temperature profile for conduction in a steady state, constant physical properties, is linear. TMAP7 was run to a time of 150,000 seconds. Steady state was assured by there being no difference at all in the last two temperature profiles. The theoretical temperature profile of A and B can be found through linear interpolation. The steady-state temperatures predicted by TMAP7, ABAQUS, and the analytical solution were found to be identical. These values can be seen in Table 13.

Table 13. Steady-state temperature $(\mathrm{K})$ distribution for composite structure

\begin{tabular}{crrr}
\hline Depth $(\mathbf{m})$ & TMAP7 & ABAQUS & Theory \\
\hline 0.00 & 600.0 & 600.0 & 600.0 \\
0.01 & 597.5 & 597.5 & 597.5 \\
0.03 & 592.5 & 592.5 & 592.5 \\
0.05 & 587.5 & 587.5 & 587.5 \\
0.07 & 582.5 & 582.5 & 582.5 \\
0.09 & 577.5 & 577.5 & 577.5 \\
0.11 & 572.5 & 572.5 & 572.5 \\
0.13 & 567.5 & 567.5 & 567.5 \\
0.15 & 562.5 & 562.5 & 562.5 \\
0.17 & 557.5 & 557.5 & 557.5 \\
0.19 & 552.5 & 552.5 & 552.5 \\
0.21 & 547.5 & 547.5 & 547.5 \\
0.23 & 542.5 & 542.5 & 542.5 \\
0.25 & 537.5 & 537.5 & 537.5 \\
0.27 & 532.5 & 532.5 & 532.5 \\
0.29 & 527.5 & 527.5 & 527.5 \\
0.31 & 522.5 & 522.5 & 522.5 \\
0.33 & 517.5 & 517.5 & 517.5 \\
0.35 & 512.5 & 512.5 & 512.5 \\
0.37 & 507.5 & 507.5 & 507.5 \\
0.39 & 502.5 & 502.5 & 502.5 \\
0.40 & 500.0 & 500.0 & 500.0 \\
0.41 & 487.5 & 487.5 & 487.5 \\
0.43 & 462.5 & 462.5 & 462.5 \\
0.45 & 437.5 & 437.5 & 437.5 \\
0.47 & 412.5 & 412.5 & 412.5 \\
0.49 & 387.5 & 387.5 & 387.5 \\
0.51 & 362.5 & 362.5 & 362.5 \\
0.53 & 337.5 & 337.5 & 337.5 \\
0.55 & 312.5 & 312.5 & 312.5 \\
0.57 & 287.5 & 287.5 & 287.5 \\
0.59 & 262.5 & 262.5 & 262.5 \\
0.61 & 237.5 & 237.5 & 237.5
\end{tabular}




\begin{tabular}{crrr}
\hline Depth $(\mathbf{m})$ & TMAP7 & ABAQUS & Theory \\
\hline 0.63 & 212.5 & 212.5 & 212.5 \\
0.65 & 187.5 & 187.5 & 187.5 \\
0.67 & 162.5 & 162.5 & 162.5 \\
0.69 & 137.5 & 137.5 & 137.5 \\
0.71 & 112.5 & 112.5 & 112.5 \\
0.73 & 87.5 & 87.5 & 87.5 \\
0.75 & 62.5 & 62.5 & 62.5 \\
0.77 & 37.5 & 37.5 & 37.5 \\
0.79 & 12.5 & 12.5 & 12.5 \\
0.80 & 0.0 & 0.0 & 0.0 \\
\hline
\end{tabular}

\subsubsection{Convective Heating (Val-1fd)}

The fourth heat transfer problem modeled was the heating of a semi-infinite slab by convection at the boundary. The slab was initially configured with a constant temperature of $100 \mathrm{~K}$ throughout the slab. A convection boundary was then activated at the surface for time, $\mathrm{t} \geq 0 \mathrm{sec}$. Incorpera and DeWitt ${ }^{10}$ give for the solution

$$
T(x, t)=T_{i}+\left(T_{\infty}-T_{i}\right)\left\{\left[\operatorname{erfc}\left(\frac{x}{2 \sqrt{t \alpha}}\right)\right]-\left[\exp \left(\frac{h x}{k}+\frac{h^{2} t \alpha}{k^{2}}\right)\right]\left[\operatorname{erfc}\left(\frac{x}{2 \sqrt{t \alpha}}+\frac{h \sqrt{t \alpha}}{k}\right)\right]\right\}
$$

where

$$
\begin{aligned}
& T_{\mathrm{i}}=\text { initial temperature }(100 \mathrm{~K}) \\
& T_{\infty}=\text { temperature of enclosure }(500 \mathrm{~K}) \\
& h=\text { conduction coefficient }\left(200 \mathrm{~W} / \mathrm{m}^{2} \mathrm{~K}\right) \\
& k=\text { thermal conductivity }(401 \mathrm{~W} / \mathrm{m} \mathrm{K}) \\
& \alpha=\text { thermal diffusivity }\left(1.17 \times 10^{-4} \mathrm{~m}^{2} / \mathrm{s}\right)
\end{aligned}
$$

The depth $x$ of $5 \mathrm{~cm}$ was used for comparison. Values of the complimentary error function were computed using a series expansion in $\mathrm{MS} \mathrm{Excel}^{\mathrm{TM}}$. The last term computed contributed less than $1.0 \times 10^{-20}$. The variance between Eq. (29) and TMAP7 was less than $0.2 \%$, for all times greater than $30 \mathrm{sec}$, as can be seen in Table 14. A graphical comparison can be seen in Figure 9. 


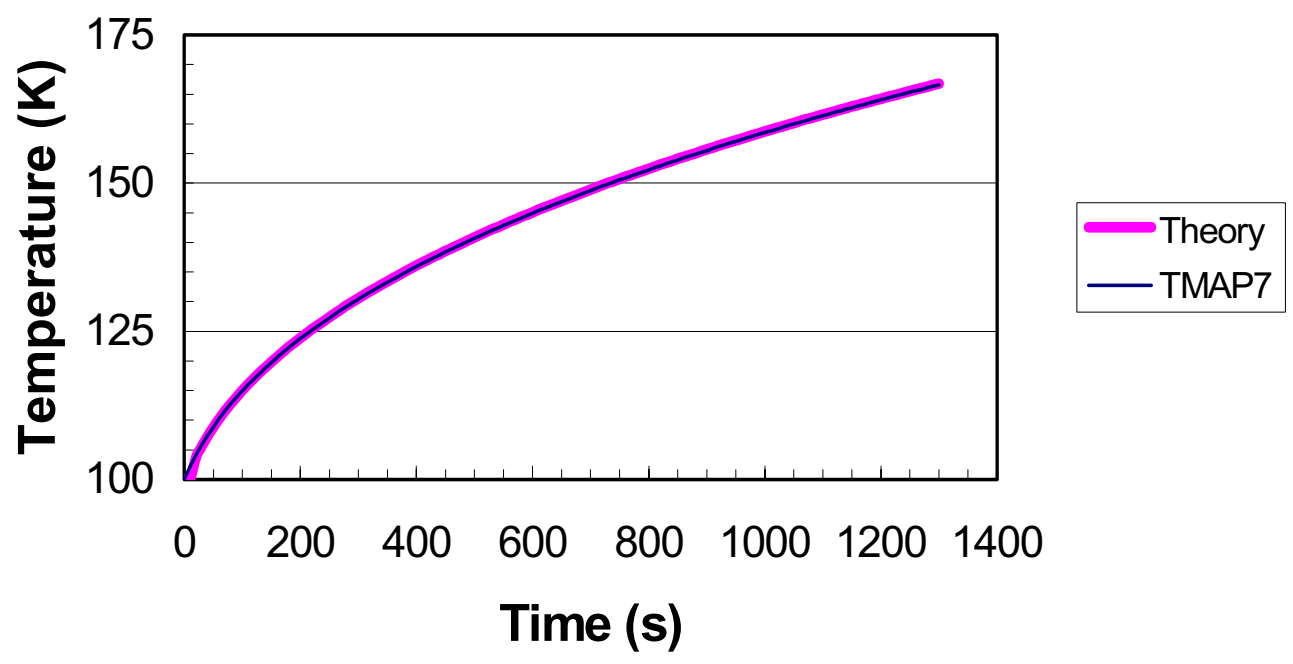

Figure 9. Convective heating at depth $5 \mathrm{~cm}$ in a semi-infinite slab (Val-1fd).

Table 14. Heating of Semi-Infinite Slab by Convection

\begin{tabular}{rrrr}
\hline Time (s) & TMAP7 & \multicolumn{1}{l}{ Theory } & Variance \\
\hline 0 & 100.000 & 100.000 & 0.000000 \\
10 & 102.140 & 100.000 & 0.021400 \\
20 & 104.060 & 103.639 & 0.004063 \\
30 & 105.800 & 105.549 & 0.002376 \\
40 & 107.390 & 107.252 & 0.001286 \\
50 & 108.860 & 108.795 & 0.000600 \\
60 & 110.230 & 110.211 & 0.000169 \\
70 & 111.510 & 111.527 & -0.000148 \\
80 & 112.720 & 112.758 & -0.000333 \\
90 & 113.860 & 113.918 & -0.000508 \\
100 & 114.940 & 115.017 & -0.000673 \\
110 & 115.980 & 116.064 & -0.000724 \\
120 & 116.970 & 117.064 & -0.000806 \\
130 & 117.920 & 118.023 & -0.000876 \\
140 & 118.840 & 118.945 & -0.000887 \\
150 & 119.720 & 119.834 & -0.000953 \\
160 & 120.580 & 120.693 & -0.000934 \\
170 & 121.410 & 121.524 & -0.000934 \\
180 & 122.210 & 122.329 & -0.000973 \\
\hline
\end{tabular}

\subsection{Problem 1g: Enclosure Reaction Problems}

Three problems were solved in TMAP7 to test its capability to handle enclosure reactions. The first model is a simple forward reaction with two reactants forming one product. In the first model, the reactants start in their stoichiometric ratio. The second problem varies from the first in that the concentrations of the reactants vary from their stoichiometric ratio. The third problem examines a series reaction. 


\subsubsection{Simple Forward Reactions (Val-1ga and Val-1gb)}

The first and second problems consider the simple chemical reaction

$$
A+B \rightarrow A B \text {. }
$$

The rate at which the concentrations change (rate of reaction) is assumed first order with respect to the concentrations of $A$ and $B$. The rate coefficient, $\mathrm{K}_{\mathrm{r}}$, is a constant for the reaction and has no spatial or time dependence. The simple forward reaction rate

$$
\frac{d C_{A B}}{d t}=R_{c}=K_{r} C_{A} C_{B}
$$

is positive if $\mathrm{AB}$ is produced and negative if $\mathrm{AB}$ is consumed in the reaction. This may also be written

$$
\frac{d C_{A B}}{\left(C_{A B}-C_{A_{0}}\right)\left(C_{A B}-C_{B_{0}}\right)}=K_{r} d t
$$

The solution for this problem is ${ }^{12}$

$$
C_{A B}=C_{B_{o}} \frac{1-\exp \left[K_{R} t\left(C_{B_{O}}-C_{A_{O}}\right)\right]}{1-\frac{C_{B_{O}}}{C_{A_{O}}} \exp \left[K_{R} t\left(C_{B_{O}}-C_{A_{O}}\right)\right]}
$$

where

$$
\begin{aligned}
& \mathrm{C}_{\mathrm{AB}}=\text { concentration of species } \mathrm{AB} \\
& C_{A_{O}}=\text { initial concentration of species A } \\
& C_{B_{O}}=\text { initial concentration of species B }
\end{aligned}
$$

If $C_{A_{o}}=C_{B_{o}}$, Eq. (33) can be simplified to

$$
C_{A B}=C_{A_{O}}-\frac{1}{\frac{1}{C_{A_{O}}}+K_{R} t} .
$$

The analytical solutions of Eqs. (33) and (34) were found and compared to the values obtained from TMAP7. Eq. (34) was solved and compared to TMAP7 for problem Val-1ga, and Eq. (33) was compared to TMAP7 for problem Val-1gb. These results are listed in Tables 15 and 16 , respectively. Figure 10 shows a graphical comparison of the two cases. The variance in each of the two cases drops below $0.2 \%$ for time, $t \geq 2 \mathrm{sec}$. 


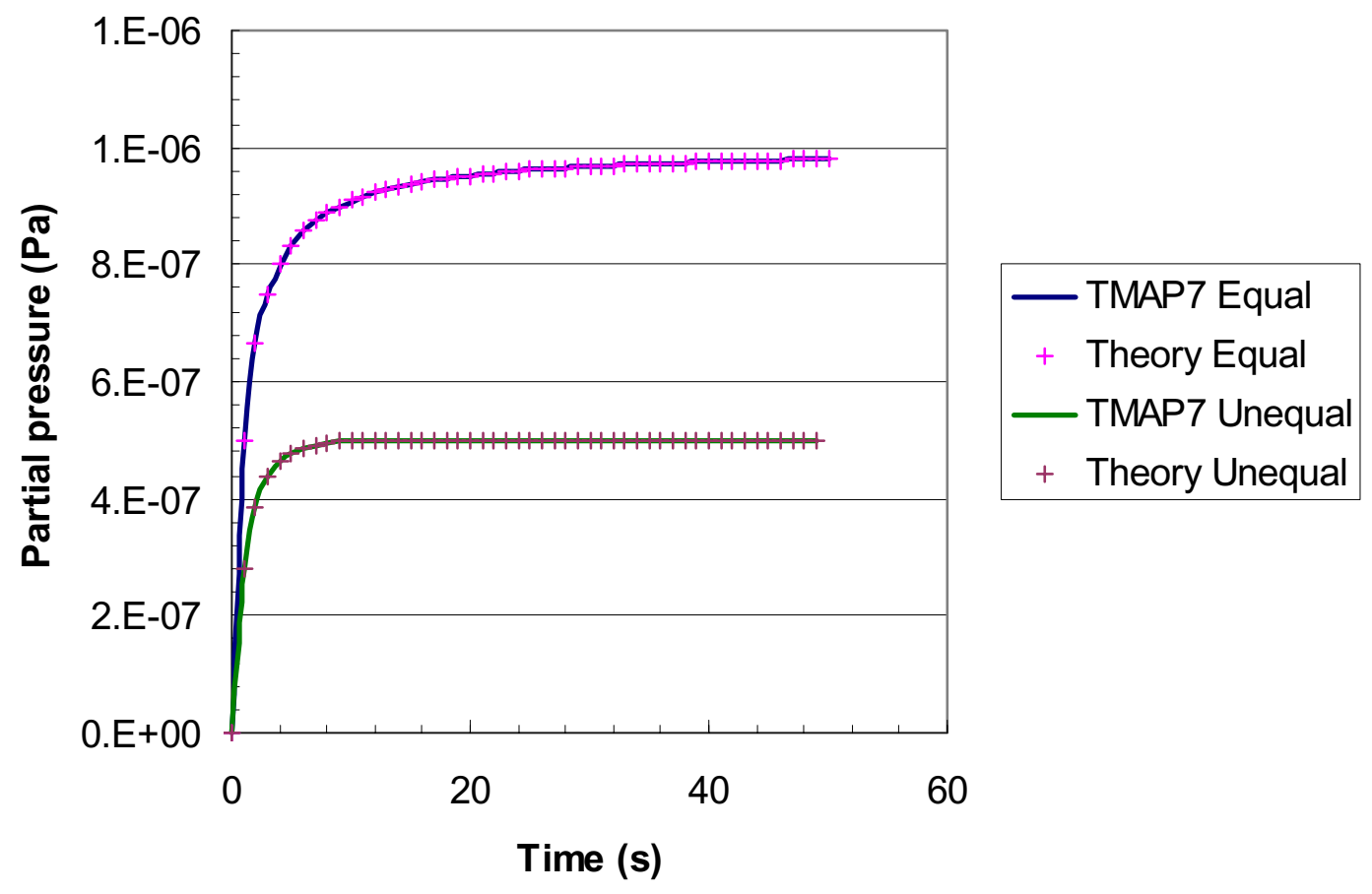

Figure 10. Production of $\mathrm{AB}$ from $\mathrm{A}$ and $\mathrm{B}$ under assumptions of equal and unequal initial reactant concentrations (Val-1ga/Val-1gb).

Table 15. Partial pressure $(\mathrm{Pa})$ of product for equal starting concentrations.

\begin{tabular}{crrr}
\hline Time (s) & TMAP7 & Theory & Variance \\
\hline 0 & $0.00 \mathrm{E}+00$ & $0.00 \mathrm{E}+00$ & 0.0000 \\
1 & $4.98 \mathrm{E}-07$ & $5.00 \mathrm{E}-07$ & -0.0037 \\
2 & $6.65 \mathrm{E}-07$ & $6.67 \mathrm{E}-07$ & -0.0020 \\
3 & $7.49 \mathrm{E}-07$ & $7.50 \mathrm{E}-07$ & -0.0013 \\
4 & $7.99 \mathrm{E}-07$ & $8.00 \mathrm{E}-07$ & -0.0010 \\
5 & $8.33 \mathrm{E}-07$ & $8.33 \mathrm{E}-07$ & -0.0008 \\
6 & $8.57 \mathrm{E}-07$ & $8.57 \mathrm{E}-07$ & -0.0006 \\
7 & $8.75 \mathrm{E}-07$ & $8.75 \mathrm{E}-07$ & -0.0005 \\
8 & $8.88 \mathrm{E}-07$ & $8.89 \mathrm{E}-07$ & -0.0005 \\
9 & $9.00 \mathrm{E}-07$ & $9.00 \mathrm{E}-07$ & -0.0004 \\
10 & $9.09 \mathrm{E}-07$ & $9.09 \mathrm{E}-07$ & -0.0004 \\
11 & $9.16 \mathrm{E}-07$ & $9.17 \mathrm{E}-07$ & -0.0003 \\
12 & $9.23 \mathrm{E}-07$ & $9.23 \mathrm{E}-07$ & -0.0003 \\
13 & $9.28 \mathrm{E}-07$ & $9.29 \mathrm{E}-07$ & -0.0003 \\
14 & $9.33 \mathrm{E}-07$ & $9.33 \mathrm{E}-07$ & -0.0003 \\
15 & $9.37 \mathrm{E}-07$ & $9.37 \mathrm{E}-07$ & -0.0003 \\
16 & $9.41 \mathrm{E}-07$ & $9.41 \mathrm{E}-07$ & -0.0002 \\
17 & $9.44 \mathrm{E}-07$ & $9.44 \mathrm{E}-07$ & -0.0002 \\
18 & $9.47 \mathrm{E}-07$ & $9.47 \mathrm{E}-07$ & -0.0002 \\
19 & $9.50 \mathrm{E}-07$ & $9.50 \mathrm{E}-07$ & -0.0002
\end{tabular}




\begin{tabular}{crrr}
\hline Time (s) & TMAP7 & Theory & Variance \\
\hline 20 & $9.52 \mathrm{E}-07$ & $9.52 \mathrm{E}-07$ & -0.0002 \\
21 & $9.54 \mathrm{E}-07$ & $9.55 \mathrm{E}-07$ & -0.0002 \\
22 & $9.56 \mathrm{E}-07$ & $9.57 \mathrm{E}-07$ & -0.0002 \\
23 & $9.58 \mathrm{E}-07$ & $9.58 \mathrm{E}-07$ & -0.0002 \\
24 & $9.60 \mathrm{E}-07$ & $9.60 \mathrm{E}-07$ & -0.0002 \\
25 & $9.61 \mathrm{E}-07$ & $9.62 \mathrm{E}-07$ & -0.0002 \\
26 & $9.63 \mathrm{E}-07$ & $9.63 \mathrm{E}-07$ & -0.0002 \\
27 & $9.64 \mathrm{E}-07$ & $9.64 \mathrm{E}-07$ & -0.0002 \\
28 & $9.65 \mathrm{E}-07$ & $9.66 \mathrm{E}-07$ & -0.0002 \\
29 & $9.66 \mathrm{E}-07$ & $9.67 \mathrm{E}-07$ & -0.0002 \\
30 & $9.68 \mathrm{E}-07$ & $9.68 \mathrm{E}-07$ & -0.0002 \\
31 & $9.69 \mathrm{E}-07$ & $9.69 \mathrm{E}-07$ & -0.0002 \\
32 & $9.70 \mathrm{E}-07$ & $9.70 \mathrm{E}-07$ & -0.0002 \\
33 & $9.70 \mathrm{E}-07$ & $9.71 \mathrm{E}-07$ & -0.0002 \\
34 & $9.71 \mathrm{E}-07$ & $9.71 \mathrm{E}-07$ & -0.0002 \\
35 & $9.72 \mathrm{E}-07$ & $9.72 \mathrm{E}-07$ & -0.0002 \\
36 & $9.73 \mathrm{E}-07$ & $9.73 \mathrm{E}-07$ & -0.0002 \\
37 & $9.74 \mathrm{E}-07$ & $9.74 \mathrm{E}-07$ & -0.0002 \\
38 & $9.74 \mathrm{E}-07$ & $9.74 \mathrm{E}-07$ & -0.0002 \\
39 & $9.75 \mathrm{E}-07$ & $9.75 \mathrm{E}-07$ & -0.0002 \\
40 & $9.75 \mathrm{E}-07$ & $9.76 \mathrm{E}-07$ & -0.0002 \\
41 & $9.76 \mathrm{E}-07$ & $9.76 \mathrm{E}-07$ & -0.0002 \\
42 & $9.77 \mathrm{E}-07$ & $9.77 \mathrm{E}-07$ & -0.0002 \\
43 & $9.77 \mathrm{E}-07$ & $9.77 \mathrm{E}-07$ & -0.0002 \\
44 & $9.78 \mathrm{E}-07$ & $9.78 \mathrm{E}-07$ & -0.0002 \\
45 & $9.78 \mathrm{E}-07$ & $9.78 \mathrm{E}-07$ & -0.0002 \\
46 & $9.79 \mathrm{E}-07$ & $9.79 \mathrm{E}-07$ & -0.0002 \\
47 & $9.79 \mathrm{E}-07$ & $9.79 \mathrm{E}-07$ & -0.0002 \\
48 & $9.79 \mathrm{E}-07$ & $9.80 \mathrm{E}-07$ & -0.0002 \\
49 & $9.80 \mathrm{E}-07$ & $9.80 \mathrm{E}-07$ & -0.0002 \\
50 & $9.80 \mathrm{E}-07$ & $9.80 \mathrm{E}-07$ & -0.0002 \\
\hline & & &
\end{tabular}


Table 16. Partial pressure $(\mathrm{Pa})$ of product for reaction with unequal starting concentrations.

\begin{tabular}{|c|c|c|c|}
\hline Time (s) & TMAP7 & Theory & Variance \\
\hline 0 & $0.00 \mathrm{E}+00$ & $0.00 \mathrm{E}+00$ & 0.0000 \\
\hline 1 & 2.82E-07 & 2.82E-07 & -0.0005 \\
\hline 2 & 3.87E-07 & 3.87E-07 & -0.0003 \\
\hline 3 & 4.37E-07 & 4.37E-07 & -0.0003 \\
\hline 4 & 4.64E-07 & 4.64E-07 & -0.0002 \\
\hline 5 & 4.79E-07 & 4.79E-07 & -0.0002 \\
\hline 6 & 4.87E-07 & 4.87E-07 & -0.0002 \\
\hline 7 & 4.92E-07 & 4.92E-07 & -0.0002 \\
\hline 8 & 4.95E-07 & 4.95E-07 & -0.0002 \\
\hline 9 & 4.97E-07 & 4.97E-07 & -0.0001 \\
\hline 10 & 4.98E-07 & 4.98E-07 & -0.0002 \\
\hline 11 & 4.99E-07 & 4.99E-07 & -0.0002 \\
\hline 12 & 4.99E-07 & 4.99E-07 & -0.0001 \\
\hline 13 & 5.00E-07 & 5.00E-07 & -0.0001 \\
\hline 14 & 5.00E-07 & 5.00E-07 & -0.0001 \\
\hline 15 & $5.00 \mathrm{E}-07$ & 5.00E-07 & -0.0001 \\
\hline 16 & $5.00 \mathrm{E}-07$ & 5.00E-07 & -0.0002 \\
\hline 17 & 5.00E-07 & 5.00E-07 & -0.0001 \\
\hline 18 & 5.00E-07 & 5.00E-07 & -0.0001 \\
\hline 19 & $5.00 \mathrm{E}-07$ & 5.00E-07 & -0.0001 \\
\hline 20 & 5.00E-07 & 5.00E-07 & -0.0001 \\
\hline 21 & 5.00E-07 & 5.00E-07 & -0.0001 \\
\hline 22 & 5.00E-07 & $5.00 \mathrm{E}-07$ & -0.0002 \\
\hline 23 & 5.00E-07 & 5.00E-07 & -0.0001 \\
\hline 24 & 5.00E-07 & 5.00E-07 & -0.0001 \\
\hline 25 & 5.00E-07 & 5.00E-07 & -0.0001 \\
\hline 26 & 5.00E-07 & 5.00E-07 & -0.0001 \\
\hline 27 & 5.00E-07 & 5.00E-07 & -0.0001 \\
\hline 28 & $5.00 \mathrm{E}-07$ & 5.00E-07 & -0.0001 \\
\hline 29 & 5.00E-07 & 5.00E-07 & -0.0001 \\
\hline 30 & 5.00E-07 & 5.00E-07 & -0.0001 \\
\hline 31 & 5.00E-07 & 5.00E-07 & -0.0001 \\
\hline 32 & $5.00 \mathrm{E}-07$ & 5.00E-07 & -0.0001 \\
\hline 33 & 5.00E-07 & 5.00E-07 & -0.0001 \\
\hline 34 & 5.00E-07 & 5.00E-07 & -0.0001 \\
\hline 35 & 5.00E-07 & 5.00E-07 & -0.0001 \\
\hline 36 & 5.00E-07 & 5.00E-07 & -0.0001 \\
\hline 37 & 5.00E-07 & 5.00E-07 & -0.0001 \\
\hline 38 & 5.00E-07 & 5.00E-07 & -0.0001 \\
\hline 39 & 5.00E-07 & 5.00E-07 & -0.0001 \\
\hline 40 & 5.00E-07 & 5.00E-07 & -0.0001 \\
\hline
\end{tabular}

\subsubsection{Series Reactions (Val-1gc)}

The third problem modeled is a set of reactions in series. The system was configured so that the enclosure initially contained only species A. At time $t \geq 0$, the reactions were allowed to proceed. The reactions that were modeled are 


$$
A \stackrel{k_{1}}{\longrightarrow} B \stackrel{k_{2}}{\longrightarrow} C \text {. }
$$

The production rate for each species (negative means consumption) is given by

$$
\begin{aligned}
& -r_{A}^{\prime}=k_{1} C_{A} \\
& r_{B}^{\prime}=k_{1} C_{A}-k_{2} C_{B} \\
& r_{C}^{\prime}=k_{2} C_{B}
\end{aligned}
$$

Fogler ${ }^{13}$ gives the concentrations of $\mathrm{A}$ and $\mathrm{B}$ as

$$
\begin{aligned}
& C_{A}=C_{A_{o}} \exp \left(-k_{1} t\right) \\
& C_{B}=k_{1} C_{A_{o}}\left(\frac{\exp \left(-k_{1} t\right)-\exp \left(-k_{2} t\right)}{k_{2}-k_{1}}\right)
\end{aligned}
$$

where

$$
\begin{aligned}
& t \quad=\text { time }(\mathrm{sec}), \\
& C_{A_{o}}=\text { initial concentration of } \mathrm{A},\left(2.415 \times 10^{14} \text { atoms } / \mathrm{m}^{3}\right) . \\
& k_{1}=\text { rate constant of reaction } 1\left(0.0125 \mathrm{~s}^{-1}\right) \\
& k_{2}=\text { rate constant of reaction } 2\left(0.0025 \mathrm{~s}^{-1}\right) .
\end{aligned}
$$

The concentration of $\mathrm{C}$ was found by applying a mass balance over the system. From the stoichiometry of this reaction it was found that

$$
C_{C}=C_{A_{o}}-C_{A}-C_{B} \text {. }
$$

The concentration values of Eqs. (39), (40), and (41) were obtained using MS Excel ${ }^{\mathrm{TM}}$ and converted to $\mathrm{Pa}$. These numbers were then compared to the partial pressure values obtained from TMAP7. The variance for the pressures of species A and B are less than $0.03 \%$ for all time. The variance of species $\mathrm{C}$, begins at around $0.1 \%$, but continually decreases as the problem time increases. The comparisons for this problem are listed in Table 17. A graphical representation is shown in Figure 11. 


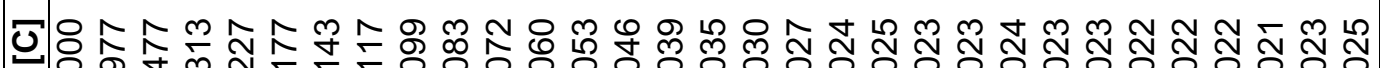

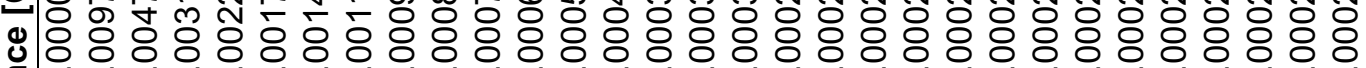

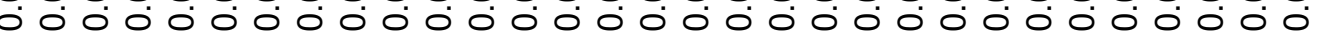

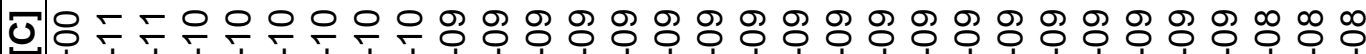

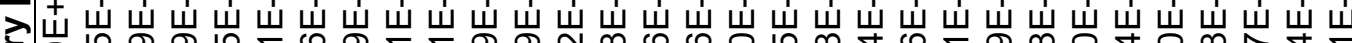

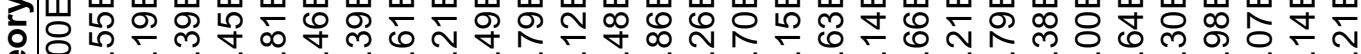

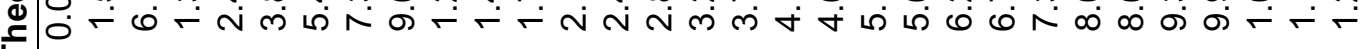

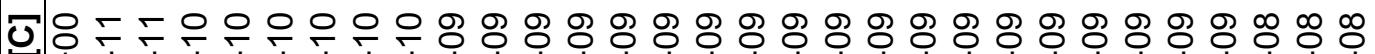

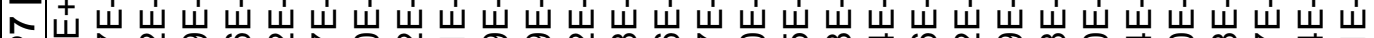
a

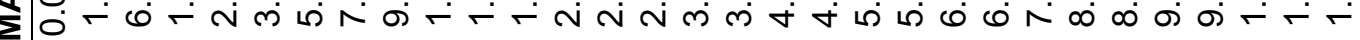

ตำ

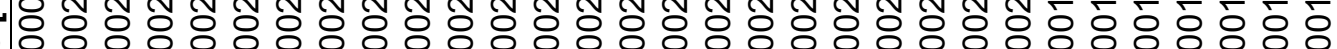

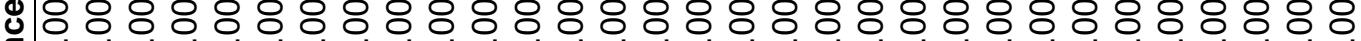
o

可 山山

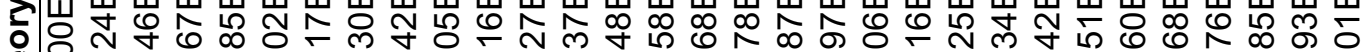
doram+orm

⿹

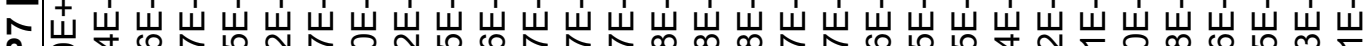

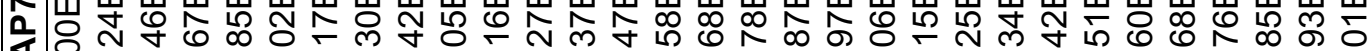

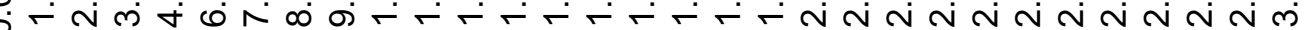

ব্ป寸ป

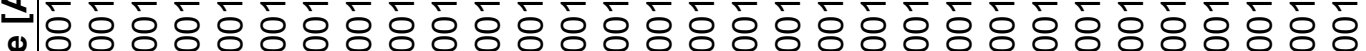

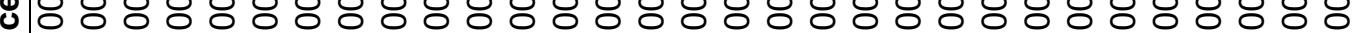
آ

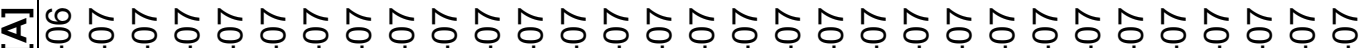

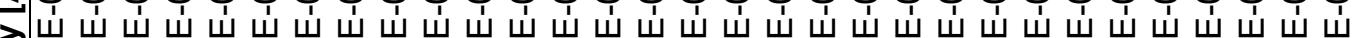
닝

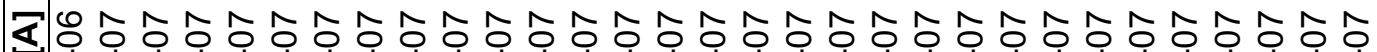

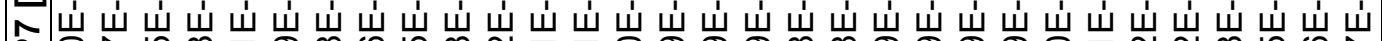
늉

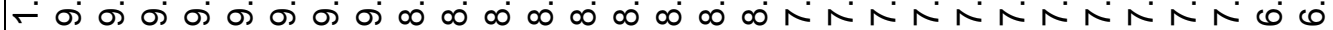




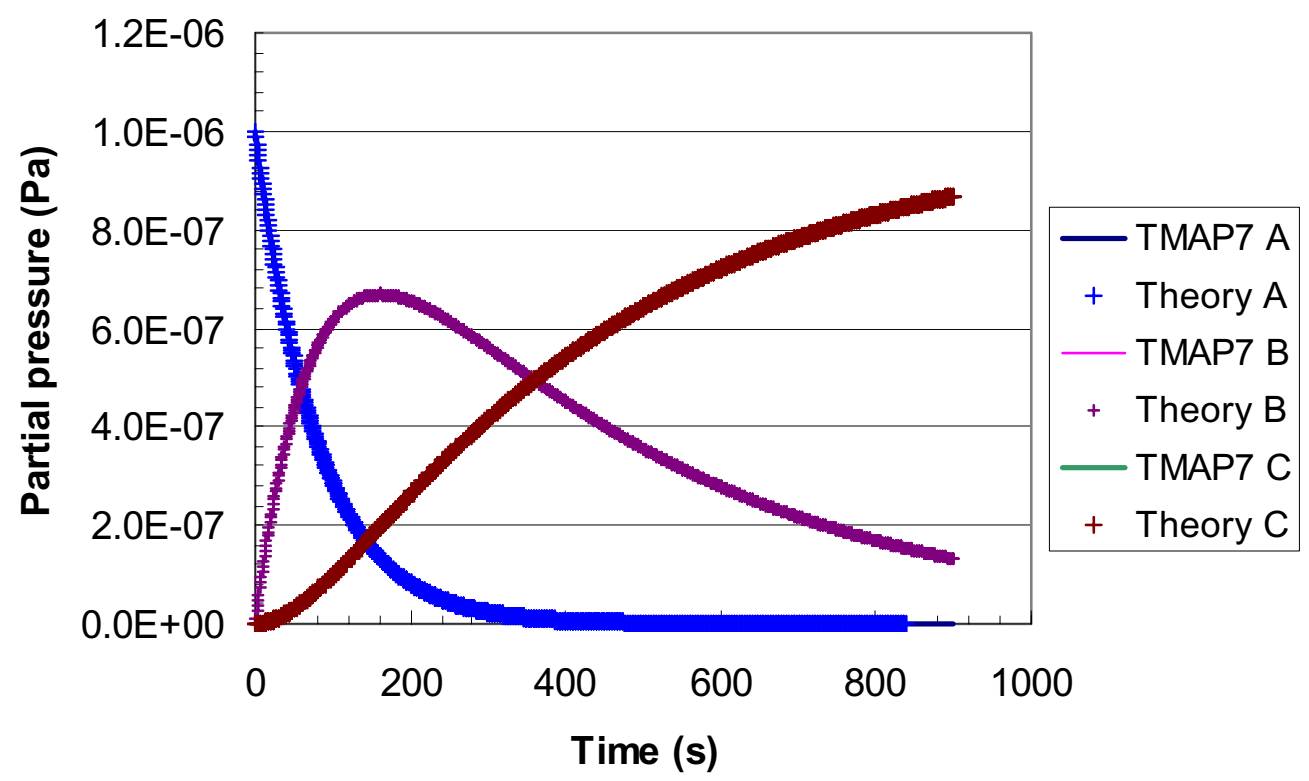

Figure 11. Partial pressures of species in series reaction (Val-1gc).

\subsection{Problem 1h: Flow Through Multiple Enclosures}

These two problems are designed to model convective flow between enclosures. The first problem models three enclosures. The first enclosure is a boundary enclosure whose concentration is constant. A convective flow goes from enclosure 1, through enclosure 2, to enclosure 3 , and then back to enclosure 1 . In the second problem, two enclosures are pre-charged with different species and a convective flow is allowed to circulate the species between the two enclosures.

\subsubsection{Three Enclosure Problem (Val-1ha)}

A system of three enclosures is modeled with flow from 1, to 2, to 3, and back to 1 . Since enclosure 1 is defined as a boundary enclosure, concentration is constant. This enclosure acts as a source and a sink. The flux, $\overline{j_{i}}$, of molecules entering into enclosure $i$ is given by

$$
\bar{j}_{i}=Q C_{1-1}
$$

where

$\mathrm{Q}=$ volumetric flow rate, common for all enclosures $\left(0.1 \mathrm{~m}^{3 /} \mathrm{sec}\right)$

$\mathrm{C}_{\mathrm{i}-1}=$ concentration of gas molecules in enclosure $\mathrm{i}-1$.

As the gas flows through the system, the number of atoms of the species of interest entering the $2^{\text {nd }}$ and $3^{\text {rd }}$ enclosures is greater than the number exiting. The concentration of that species in the enclosures rises towards the concentration in enclosure 1 . The rate of change of the concentration of this species in the $2^{\text {nd }}$ and $3^{\text {rd }}$ enclosures can be modeled as follows 


$$
\begin{aligned}
& \frac{\partial C_{2}}{\partial t}=\frac{Q\left(C_{1}-C_{2}\right)}{V_{2}} \\
& \frac{\partial C_{3}}{\partial t}=\frac{Q\left(C_{2}-C_{3}\right)}{V_{3}}
\end{aligned}
$$

The solution of this set of simultaneous equations with the initial condition that $C_{2}=C_{3}=0$ is

$$
C_{2}=C_{1}\left[1-\exp \left(-\frac{Q t}{V_{2}}\right)\right]
$$

and, if $\mathrm{V}_{2}=\mathrm{V}_{3}$,

$$
C_{3}=C_{1}\left[1-\left(1+\frac{Q t}{V_{2}}\right) \exp \left(-\frac{Q t}{V_{2}}\right)\right]
$$

Otherwise $\mathrm{C}_{3}$ is given by

$$
C_{3}=C_{1}\left[1-\frac{V_{2}}{V_{2}-V_{3}} \exp \left(-\frac{Q t}{V_{2}}\right)+\frac{V_{3}}{V_{2}-V_{3}} \exp \left(-\frac{Q t}{V_{3}}\right)\right]
$$

In this problem, the following values were used to solve Eqs. (44) and (45),

$$
\begin{aligned}
& V_{2}=V_{3}=1 \mathrm{~m}^{3}, \\
& C_{1}=2.3904 \text { atoms } / \mathrm{m}^{3}, \\
& Q=0.1 \mathrm{~m}^{3} / \mathrm{sec} .
\end{aligned}
$$

The values of Eqs. (44) and (45), were converted to partial pressures and solved using MS Excel and are compared with the values obtained from TMAP7 in Table 18 and Figure 12. The variance for Enclosure 2 is less than $0.1 \%$ for all time, while the variance for Enclosure 3 is always below $0.3 \%$, and both seem to be random with time.

\subsubsection{Equilibrating Enclosures (Val-1hb)}

The second flow problem is setup as a system of two enclosures, each with a volume of $1 \mathrm{~m}^{3}$, with flow from enclosures 1 to 2 , and 2 to 1 . Enclosure 1 is initially charged with tritium (T) and enclosure 2 is pre-charged with deuterium (D), each at $1 \mathrm{~Pa}$. The concentration change rates for this system are given by the following for species $\mathrm{T}$.

$$
\begin{aligned}
& \frac{d C_{T_{1}}}{d t}=\frac{Q}{V}\left(C_{T_{2}}-C_{T_{1}}\right) \\
& \frac{d C_{T_{2}}}{d t}=\frac{Q}{V}\left(C_{T_{1}}-C_{T_{2}}\right)
\end{aligned}
$$

and for species D

Table 18. Concentration profiles of enclosures 2 and 3 with convective flow. 


\begin{tabular}{|c|c|c|c|c|c|c|}
\hline \multirow[b]{2}{*}{ Time (s) } & \multicolumn{3}{|c|}{ Enclosure 2} & \multicolumn{3}{|c|}{ Enclosure 3} \\
\hline & TMAP7 & Theory & Var 1 & TMAP7 & Theory & Var 1 \\
\hline 0 & $0.00 \mathrm{E}+00$ & $0.00 \mathrm{E}+00$ & 0 & $0.00 \mathrm{E}+00$ & $0.00 \mathrm{E}+00$ & 0 \\
\hline 1 & $9.51 \mathrm{E}-02$ & $9.52 \mathrm{E}-02$ & -0.00021 & $4.68 \mathrm{E}-03$ & 4.68E-03 & 0.00070 \\
\hline 2 & 1.81E-01 & 1.81E-01 & -0.00011 & 1.75E-02 & 1.75E-02 & 0.00039 \\
\hline 3 & $2.59 \mathrm{E}-01$ & $2.59 \mathrm{E}-01$ & -0.00012 & 3.69E-02 & 3.69E-02 & 0.00018 \\
\hline 4 & 3.30E-01 & 3.30E-01 & -0.00030 & $6.15 \mathrm{E}-02$ & 6.16E-02 & -0.00026 \\
\hline 5 & 3.93E-01 & 3.93E-01 & -0.00038 & 9.02E-02 & 9.02E-02 & -0.00049 \\
\hline 6 & 4.51E-01 & 4.51E-01 & -0.00042 & $1.22 \mathrm{E}-01$ & $1.22 \mathrm{E}-01$ & -0.00067 \\
\hline 7 & 5.03E-01 & 5.03E-01 & -0.00045 & 1.56E-01 & $1.56 \mathrm{E}-01$ & -0.00074 \\
\hline 8 & 5.51E-01 & 5.51E-01 & -0.00026 & 1.91E-01 & 1.91E-01 & -0.00030 \\
\hline 9 & 5.93E-01 & 5.93E-01 & -0.00007 & $2.28 \mathrm{E}-01$ & 2.28E-01 & 0.00010 \\
\hline 10 & 6.32E-01 & 6.32E-01 & 0.00005 & 2.64E-01 & 2.64E-01 & 0.00041 \\
\hline 11 & 6.67E-01 & 6.67E-01 & 0.00015 & 3.01E-01 & 3.01E-01 & 0.00063 \\
\hline 12 & 6.99E-01 & 6.99E-01 & 0.00021 & 3.38E-01 & 3.37E-01 & 0.00079 \\
\hline 13 & 7.28E-01 & 7.27E-01 & 0.00025 & 3.74E-01 & 3.73E-01 & 0.00089 \\
\hline 14 & 7.54E-01 & 7.53E-01 & 0.00027 & 4.09E-01 & 4.08E-01 & 0.00099 \\
\hline 15 & 7.77E-01 & 7.77E-01 & 0.00030 & 4.43E-01 & 4.42E-01 & 0.00105 \\
\hline 16 & 7.98E-01 & 7.98E-01 & 0.00006 & 4.75E-01 & 4.75E-01 & 0.00042 \\
\hline 17 & 8.17E-01 & 8.17E-01 & -0.00015 & 5.07E-01 & 5.07E-01 & -0.00015 \\
\hline 18 & 8.34E-01 & 8.35E-01 & -0.00031 & 5.37E-01 & 5.37E-01 & -0.00058 \\
\hline 19 & $8.50 \mathrm{E}-01$ & 8.50E-01 & -0.00044 & 5.66E-01 & 5.66E-01 & -0.00096 \\
\hline 20 & 8.64E-01 & 8.65E-01 & -0.00054 & 5.93E-01 & 5.94E-01 & -0.00125 \\
\hline 21 & 8.77E-01 & 8.78E-01 & -0.00061 & 6.19E-01 & $6.20 \mathrm{E}-01$ & -0.00149 \\
\hline 22 & 8.89E-01 & 8.89E-01 & -0.00066 & 6.44E-01 & 6.45E-01 & -0.00169 \\
\hline 23 & 8.99E-01 & $9.00 \mathrm{E}-01$ & -0.00070 & 6.68E-01 & 6.69E-01 & -0.00183 \\
\hline 24 & 9.09E-01 & 9.09E-01 & -0.00072 & 6.90E-01 & 6.92E-01 & -0.00194 \\
\hline 25 & $9.17 \mathrm{E}-01$ & $9.18 \mathrm{E}-01$ & -0.00074 & $7.11 \mathrm{E}-01$ & 7.13E-01 & -0.00202 \\
\hline 26 & $9.25 \mathrm{E}-01$ & $9.26 \mathrm{E}-01$ & -0.00060 & 7.31E-01 & 7.33E-01 & -0.00160 \\
\hline 27 & 9.32E-01 & 9.33E-01 & -0.00036 & 7.51E-01 & 7.51E-01 & -0.00084 \\
\hline 28 & 9.39E-01 & 9.39E-01 & -0.00016 & 7.69E-01 & 7.69E-01 & -0.00018 \\
\hline 29 & $9.45 \mathrm{E}-01$ & $9.45 \mathrm{E}-01$ & 0.00000 & 7.86E-01 & 7.85E-01 & 0.00037 \\
\hline 30 & 9.50E-01 & $9.50 \mathrm{E}-01$ & 0.00012 & 8.02E-01 & 8.01E-01 & 0.00083 \\
\hline 31 & 9.55E-01 & 9.55E-01 & 0.00023 & 8.16E-01 & $8.15 E-01$ & 0.00120 \\
\hline 32 & $9.60 \mathrm{E}-01$ & $9.59 \mathrm{E}-01$ & 0.00030 & 8.30E-01 & 8.29E-01 & 0.00152 \\
\hline 33 & 9.63E-01 & 9.63E-01 & 0.00036 & 8.43E-01 & 8.41E-01 & 0.00178 \\
\hline 34 & 9.67E-01 & 9.67E-01 & 0.00041 & $8.55 \mathrm{E}-01$ & 8.53E-01 & 0.00197 \\
\hline 35 & $9.70 \mathrm{E}-01$ & $9.70 \mathrm{E}-01$ & 0.00043 & 8.66E-01 & 8.64E-01 & 0.00213 \\
\hline 36 & 9.73E-01 & $9.73 E-01$ & 0.00045 & 8.76E-01 & 8.74E-01 & 0.00224 \\
\hline 37 & 9.76E-01 & $9.75 \mathrm{E}-01$ & 0.00036 & 8.86E-01 & 8.84E-01 & 0.00192 \\
\hline 38 & $9.78 \mathrm{E}-01$ & $9.78 \mathrm{E}-01$ & 0.00010 & 8.93E-01 & 8.93E-01 & 0.00091 \\
\hline 39 & 9.80E-01 & $9.80 \mathrm{E}-01$ & -0.00010 & 9.01E-01 & 9.01E-01 & 0.00004 \\
\hline 40 & 9.81E-01 & 9.82E-01 & -0.00028 & 9.08E-01 & 9.08E-01 & -0.00070 \\
\hline
\end{tabular}




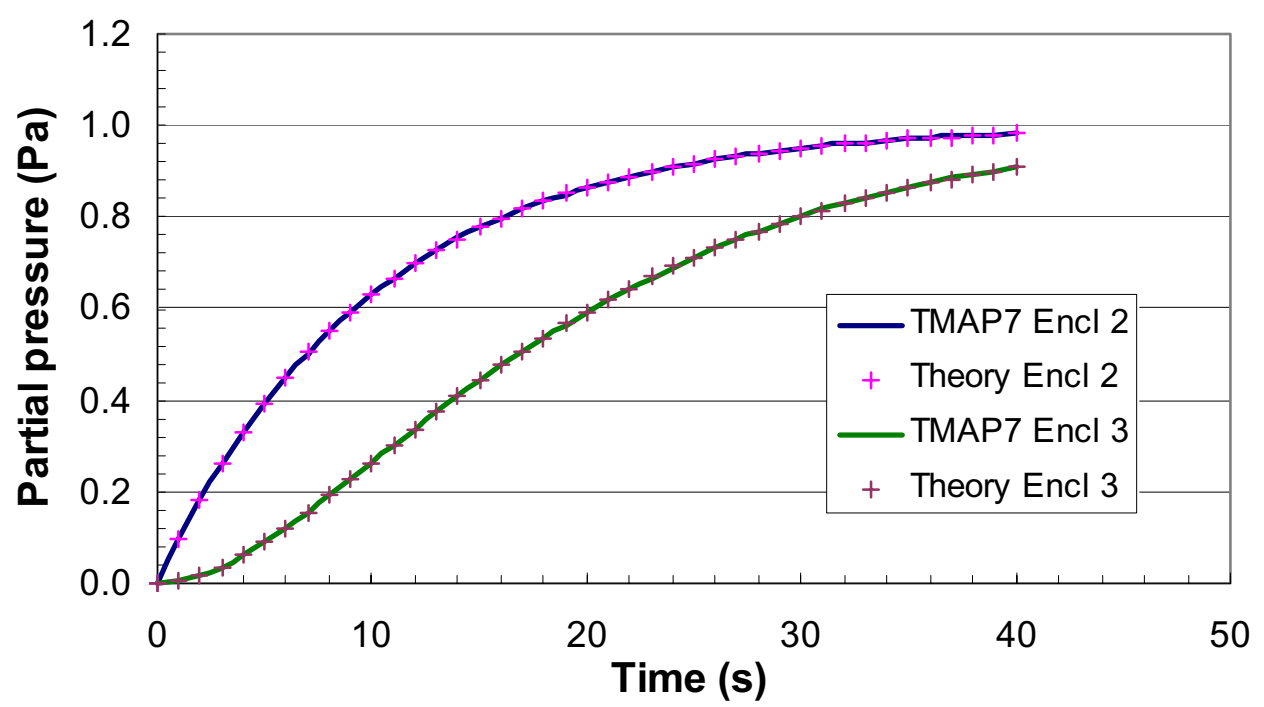

Figure 12. Concentration history of sequentially coupled enclosures (Val-1ha).

$$
\begin{aligned}
& \frac{d C_{D_{1}}}{d t}=\frac{Q}{V}\left(C_{D_{2}}-C_{D_{1}}\right) \\
& \frac{d C_{D_{2}}}{d t}=\frac{Q}{V}\left(C_{D_{1}}-C_{D_{2}}\right)
\end{aligned}
$$

where

$$
\begin{aligned}
& \mathrm{Q}=\text { volumetric flow }\left(0.1 \mathrm{~m}^{3} / \mathrm{s}\right) \\
& \mathrm{V}=\text { volume }\left(1 \mathrm{~m}^{3}\right) \\
& C_{T_{i}}=\text { concentration of tritium in Enclosure } i \\
& C_{D_{i}}=\text { concentration of deuterium in Enclosure } i
\end{aligned}
$$

A mass balance on the system, gives a relationship between the concentration of species in Enclosure 1 and Enclosure 2.

$$
C_{T_{1}}=C_{T_{1}}^{o}-C_{T_{2}}
$$

Now by substituting Eq. (49) into the first of Eqs. (48), the solution is given by

$$
C_{T_{1}}=\frac{C_{T_{1}}^{o}}{2}\left[1+\exp \left(-\frac{2 Q}{V} t\right)\right]
$$

where $C_{T_{1}}^{o}=$ initial concentration of tritium in Enclosure 1.

It is recognized that for the same initial starting conditions for deuterium, except different initial pressures ( $1 \mathrm{~Pa}$ in enclosure 2 and $0 \mathrm{~Pa}$ in enclosure 1$)$, the following will be true 


$$
\begin{aligned}
& C_{D_{2}}=C_{T_{1}} \\
& C_{D_{1}}=C_{T_{2}}
\end{aligned}
$$

Eq. (50) was solved in Excel ${ }^{\mathrm{TM}}$ and compared with the values obtained from TMAP7. These values, presented as partial pressures, are listed in Table 19 and shown graphically in Figure 13.

Table 19. Concentration of tritium in convective flow between two enclosures

\begin{tabular}{|c|c|c|c|c|c|c|}
\hline & \multicolumn{3}{|c|}{ Enclosure 1 } & \multicolumn{3}{c|}{ Enclosure 2 } \\
\hline Time (s) & TMAP7 & Theory & Variance & TMAP7 & Theory & Variance \\
\hline 0 & 1.000 & 1.000 & 0.00014 & 0.000 & 0.000 & 0.00000 \\
1 & 0.909 & 0.909 & 0.00014 & 0.091 & 0.091 & 0.00024 \\
2 & 0.835 & 0.835 & 0.00014 & 0.165 & 0.165 & 0.00012 \\
3 & 0.774 & 0.774 & 0.00012 & 0.226 & 0.226 & 0.00024 \\
4 & 0.725 & 0.725 & 0.00008 & 0.275 & 0.275 & 0.00035 \\
5 & 0.684 & 0.684 & 0.00003 & 0.316 & 0.316 & 0.00038 \\
6 & 0.651 & 0.651 & 0.00006 & 0.349 & 0.349 & 0.00029 \\
7 & 0.623 & 0.623 & 0.00016 & 0.377 & 0.377 & 0.00011 \\
8 & 0.601 & 0.601 & 0.00025 & 0.399 & 0.399 & 0.00000 \\
9 & 0.582 & 0.583 & 0.00029 & 0.417 & 0.417 & -0.00007 \\
10 & 0.567 & 0.568 & 0.00033 & 0.432 & 0.432 & -0.00009 \\
11 & 0.555 & 0.555 & 0.00022 & 0.445 & 0.445 & 0.00006 \\
12 & 0.545 & 0.545 & 0.00007 & 0.455 & 0.455 & 0.00024 \\
13 & 0.537 & 0.537 & -0.00002 & 0.463 & 0.463 & 0.00035 \\
14 & 0.530 & 0.530 & -0.00010 & 0.469 & 0.470 & 0.00042 \\
15 & 0.525 & 0.525 & -0.00013 & 0.475 & 0.475 & 0.00046 \\
16 & 0.520 & 0.520 & -0.00015 & 0.479 & 0.480 & 0.00046 \\
17 & 0.517 & 0.517 & 0.00007 & 0.483 & 0.483 & 0.00021 \\
18 & 0.514 & 0.514 & 0.00024 & 0.486 & 0.486 & 0.00006 \\
19 & 0.511 & 0.511 & 0.00032 & 0.489 & 0.489 & -0.00005 \\
20 & 0.509 & 0.509 & 0.00039 & 0.491 & 0.491 & -0.00012 \\
21 & 0.507 & 0.507 & 0.00043 & 0.493 & 0.493 & -0.00014 \\
22 & 0.506 & 0.506 & 0.00037 & 0.494 & 0.494 & -0.00008 \\
23 & 0.505 & 0.505 & 0.00015 & 0.495 & 0.495 & 0.00015 \\
24 & 0.504 & 0.504 & 0.00001 & 0.496 & 0.496 & 0.00029 \\
25 & 0.503 & 0.503 & -0.00010 & 0.496 & 0.497 & 0.00038 \\
26 & 0.503 & 0.503 & -0.00016 & 0.497 & 0.497 & 0.00045 \\
27 & 0.502 & 0.502 & -0.00018 & 0.498 & 0.498 & 0.00049 \\
28 & 0.502 & 0.502 & -0.00020 & 0.498 & 0.498 & 0.00048 \\
29 & 0.502 & 0.502 & -0.00001 & 0.498 & 0.498 & 0.00031 \\
30 & 0.501 & 0.501 & 0.00016 & 0.499 & 0.499 & 0.00012 \\
\hline & & & & & & \\
\hline
\end{tabular}




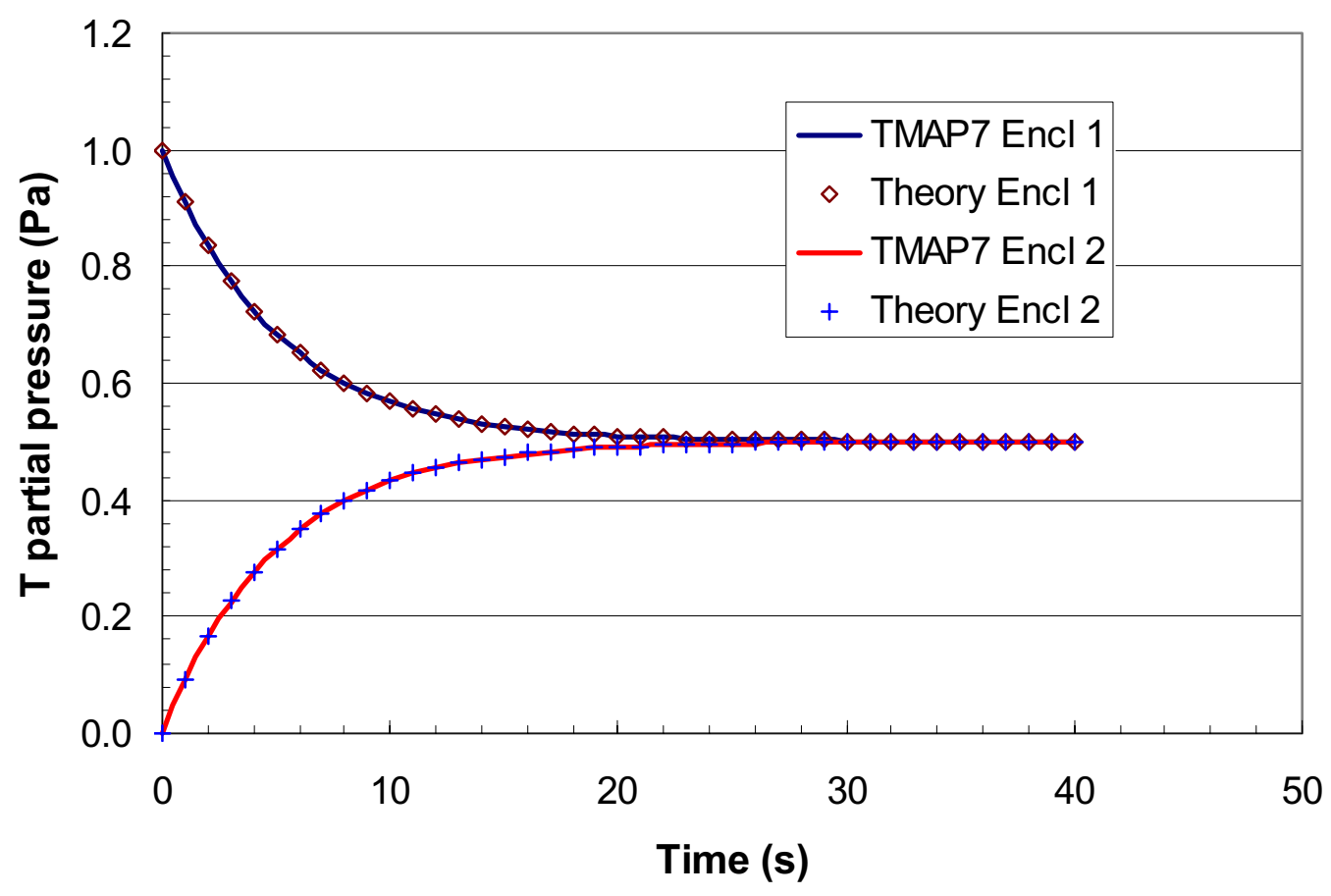

Figure 13. Tritium concentration equilibration in two communicating enclosures (Val-1hb).

\subsection{Problem 1i: Species Equilibration on a Reactive Surface}

When two species can react on a surface to form a third, it is possible to predict the rate at which equilibration between the species will occur. For example, consider the reaction between two isotopic species

$$
A_{2}+B_{2} \leftrightarrow 2 A B
$$

\subsubsection{Ratedep Conditions (Val-1ia, Val-1ib)}

The expression (derived in Appendix A) for the rate of formation of $A B$ when the conversion rate at the surface is high is

$$
P_{A B}=\frac{2 P_{A_{2}}^{0} P_{B_{2}}^{0}}{P_{A_{2}}^{0}+P_{B_{2}}^{0}}\left[1-\exp \left(-\frac{S K_{d} k T}{V} t\right)\right]
$$

Here

$P_{A_{2}}^{o}=$ initial partial pressure of species $\mathrm{A}_{2}$ in the enclosure

$P_{B_{2}}^{o}=$ initial partial pressure of species $\mathrm{B}_{2}$ in the enclosure

$S \quad=$ surface area available for reaction

$K_{d} \quad=$ dissociation coefficient of both $A_{2}$ and $B_{2}$ on the surface 


$$
\begin{aligned}
\mathrm{k} & =\text { Boltzmann's constant } \\
T & =\text { temperature } \\
V & =\text { enclosure volume }
\end{aligned}
$$

In this case $A$ was taken as protium, and $B$ was deuterium. $S$ was assumed to be $0.0025 \mathrm{~m}^{2}$, $K_{d}$ for $M=2$ amu is $1.85804 \mathrm{E}+24 / \sqrt{T}$ atom $/ \mathrm{m}^{2} / \mathrm{s}, T$ was $1,000 \mathrm{~K}$, and $V$ was $1 \mathrm{~m}^{3} . P_{A_{2}}^{o}$ and $P_{B_{2}}^{o}$ were first assumed equal at $1.0 \times 10^{4} \mathrm{~Pa}$ and then $P_{B_{2}}^{o}$ was assumed to be increased to $1.0 \times 10^{5}$ $\mathrm{Pa}$. Figure 14 shows the comparison of TMAP7 with the theory for the case of equal starting partial pressures, and Figure 15 shows the comparison for the unequal starting partial pressure case. Variance was less than $1 \%$ for all times and generally less than $0.1 \%$.

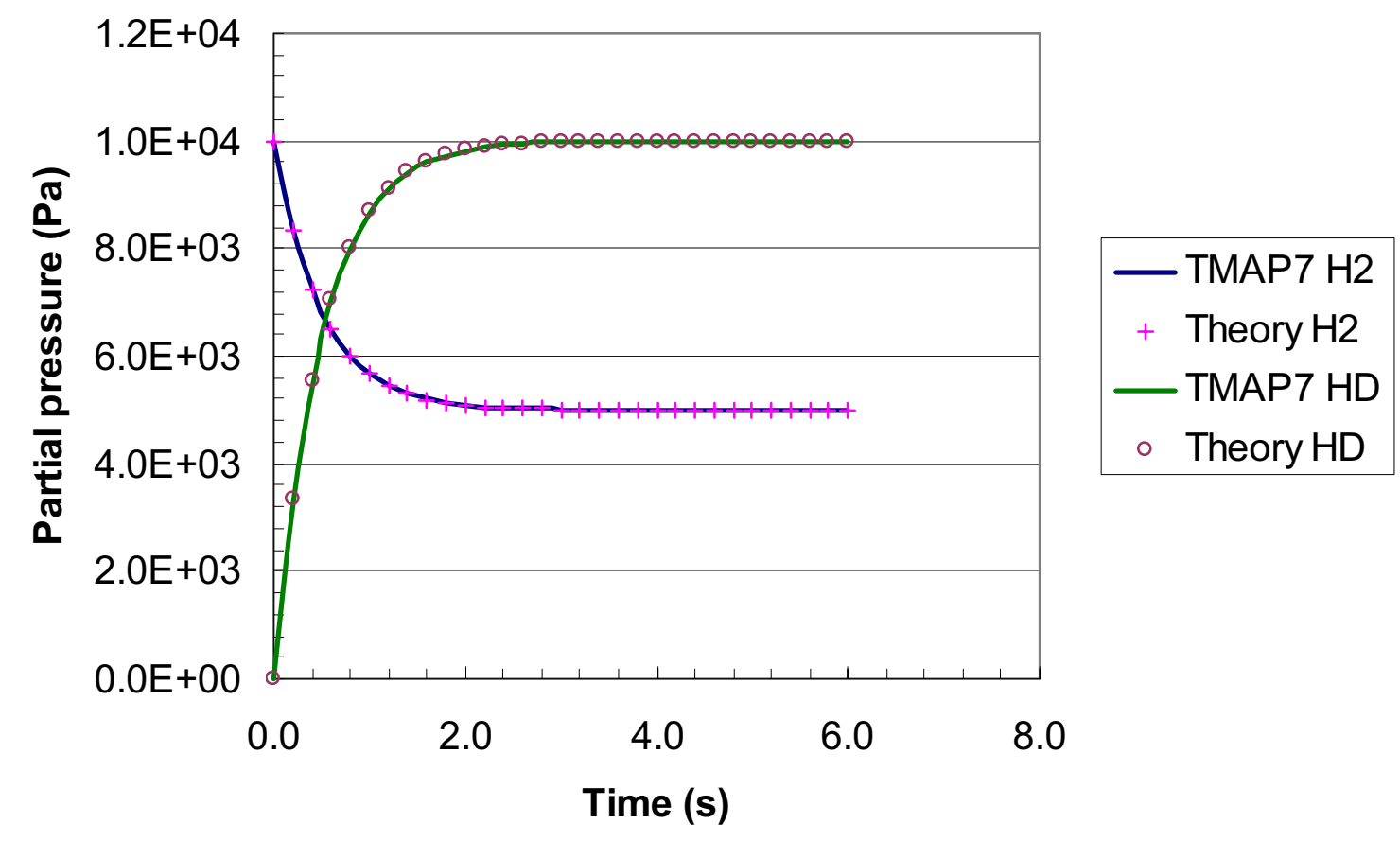

Figure 14. Equilibration of $\mathrm{H}_{2}$ with $\mathrm{D}_{2}$ to form $\mathrm{HD}$ on a tungsten surface under the assumption of equal starting partial pressures for the reactants and ratedep boundary conditions (Val-1ia). 


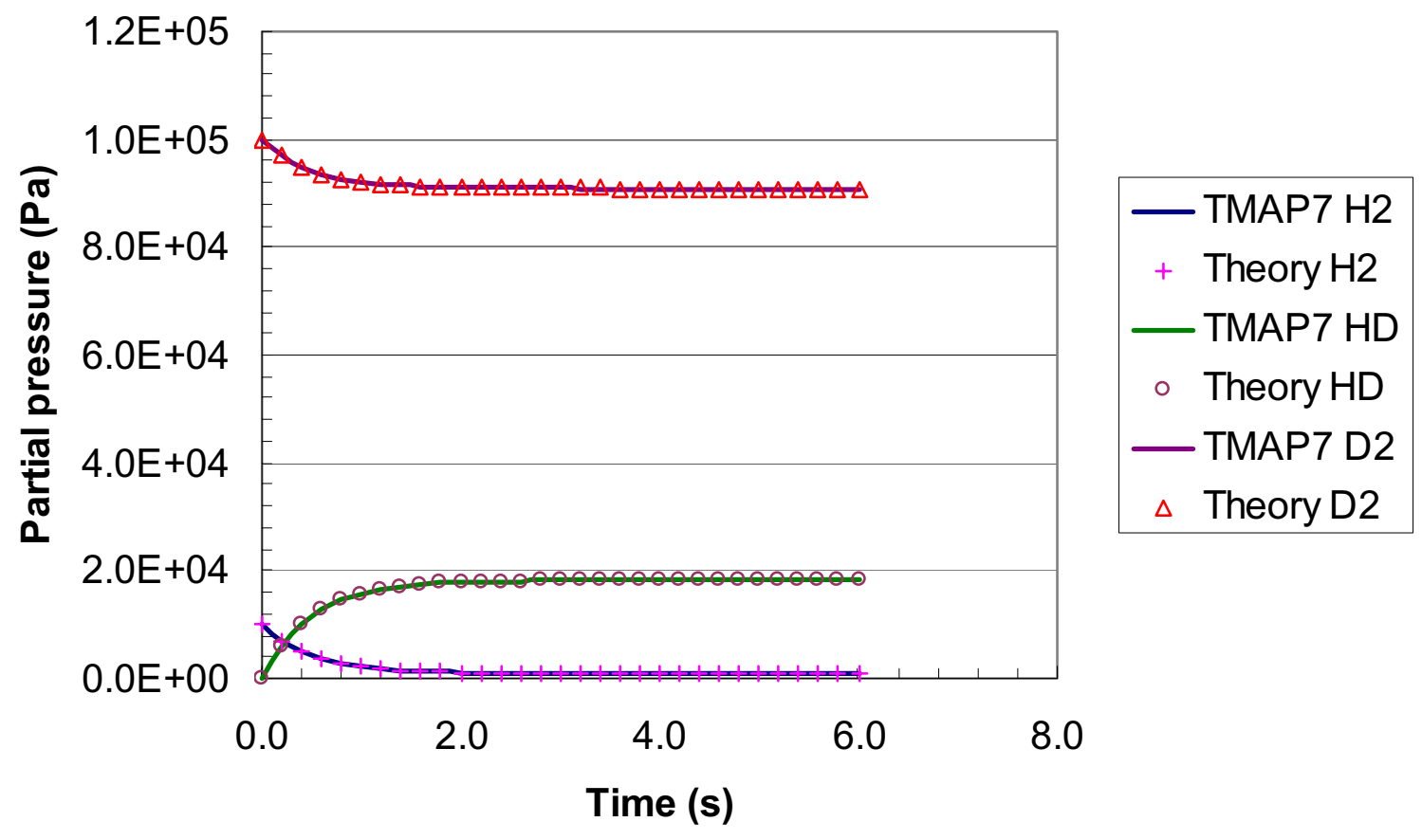

Figure 15. Chemical equilibration of $\mathrm{H}_{2}$ and $\mathrm{D}_{2}$ to $\mathrm{HD}$ on a tungsten surface with unequal starting partial pressures and ratedep boundary conditions (Val-1ib).

\subsubsection{Surfdep Conditions (Val-1ic, Val-1id)}

When surface processes are governed by activation energies with dissociation and recombination considered explicitly, surfdep boundary conditions govern. As explained in Appendix A, the equation for transient pressure of $A B$ given starting pressures of $A_{2}$ and $B_{2}$ is

$$
P_{A B}=\frac{2 P_{A_{2}}^{0} P_{B_{2}}^{0}}{P_{A_{2}}^{0}+P_{B_{2}}^{0}}\left[1-\exp \left(-\frac{t}{\tau}\right)\right]
$$

Where

$$
\tau=\frac{V\left(\hat{K}_{r}+K_{b}\right)}{S k T \hat{K}_{d} K_{b}}
$$

and for molecular mass $M$,

$$
K_{d}=\frac{1}{\sqrt{2 \pi M k T}}
$$

$K_{b}$ is a thermally activated dissociation coefficient, assumed to be given by a Boltzmann equation with activation energy $E_{b}$. and Debye frequency $v_{o}$. 


$$
K_{b}=v_{o} \exp \left(-\frac{E_{b}}{k T}\right)
$$

Molecules escape from the surface at rate

$$
\hat{K}_{r}=\frac{v_{o}}{6} \exp \left(\frac{E_{c}-E_{x}}{k T}\right)
$$

Here, in addition to variables previously defined, $E_{c}$ is the surface binding energy, and the factor of 6 accounts for the probability that on any given vibration, the direction of the phonon is away from the material surface.

The first of the surfep cases uses equal starting pressures of $1.0 \times 10^{4} \mathrm{~Pa}$ of $\mathrm{H}_{2}$ and $\mathrm{D}_{2}$ and no HD. In this case, $E_{x}$ was specified to $0.05 \mathrm{eV}, E_{c}$ was $-0.01 \mathrm{eV}$ and the dissociation energy was taken as zero, meaning that attempts at the Debye frequency all succeed. Temperature was again $1,000 \mathrm{~K}$, the surface area was a $5-\mathrm{cm} \times 5-\mathrm{cm}$ square, and the enclosure volume was $1.0 \mathrm{~m}^{3}$. Comparison of TMAP7 code results with the theoretical values is made in Figure 16. Corresponding results for unequal starting pressures are shown in Figure 17.

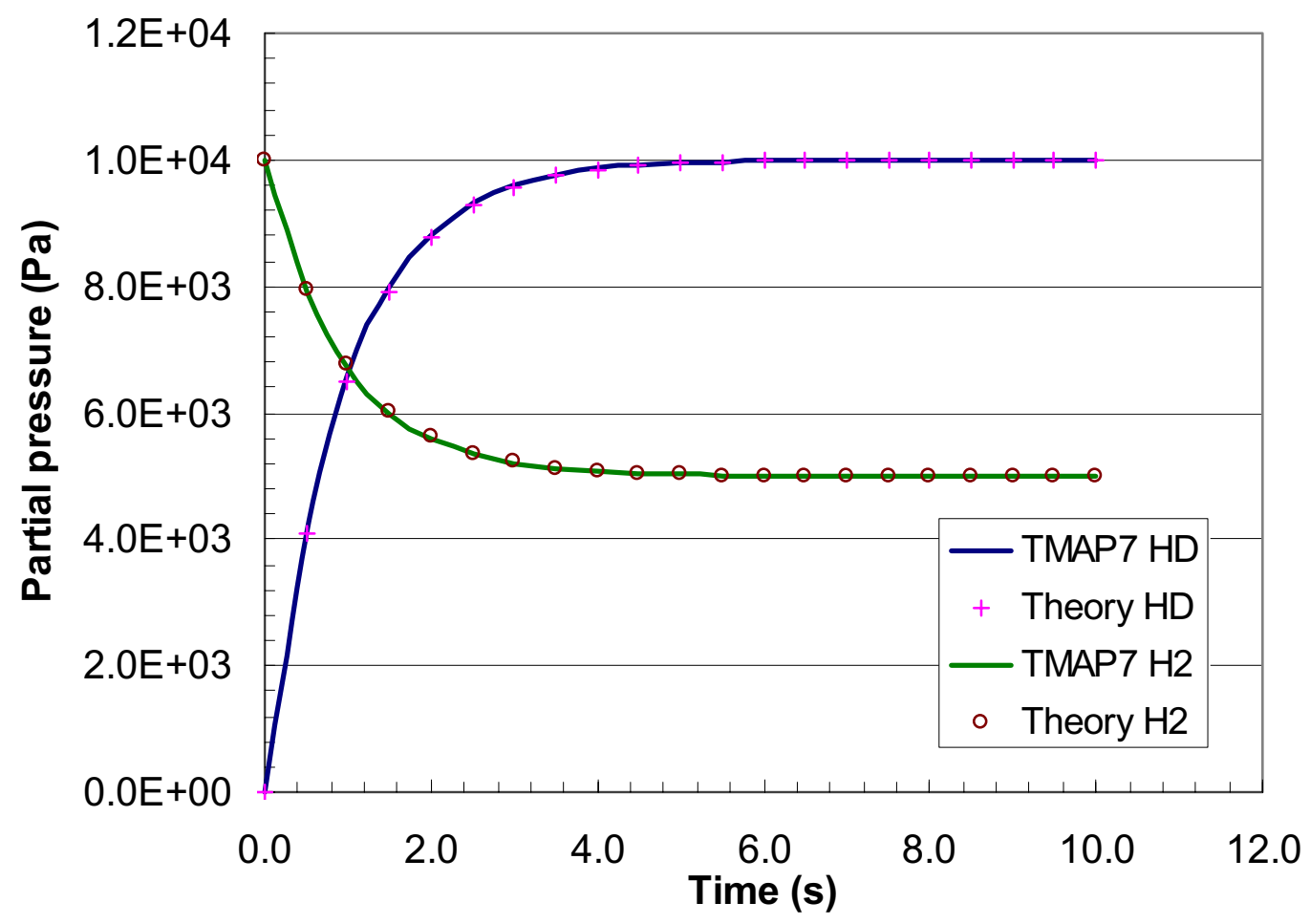

Figure 16. Chemical equilibration of $\mathrm{H}_{2}$ and $\mathrm{D}_{2}$ to form $\mathrm{HD}$ under surfdep boundary conditions with equal starting pressures (Val-1ic). 


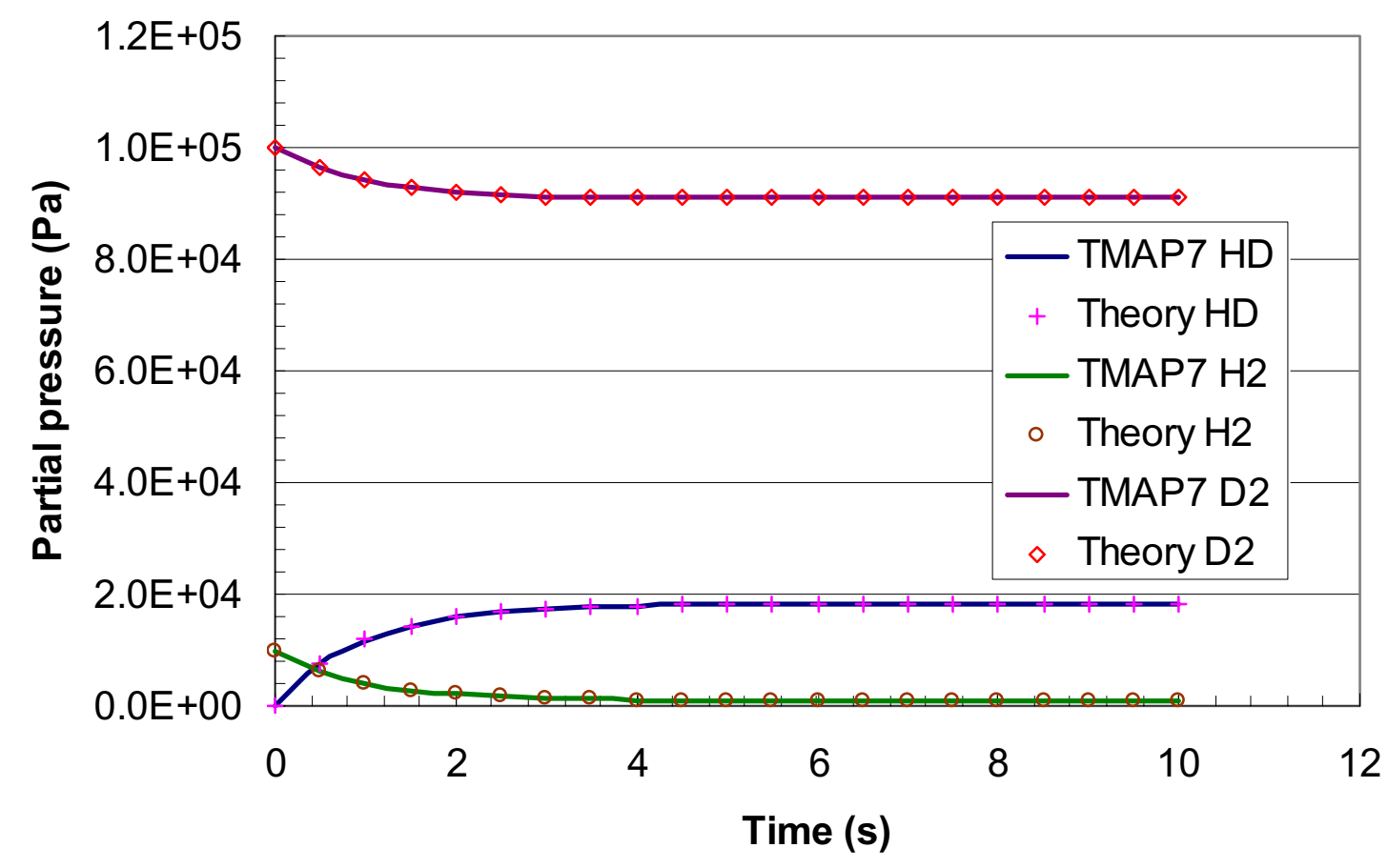

Figure 17. Chemical equilibration of $\mathrm{H}_{2}$ and $\mathrm{D}_{2}$ to form $\mathrm{HD}$ under surfdep boundary conditions with unequal starting pressures (Val-1id).

\subsection{Problem 1j: Radioactive Decay}

Two problems were run to demonstrate tritium decay, though any other isotope could have been chosen. The first is simple decay of mobile species in a slab. The second is decay of trapped atoms in a similar slab but with a distributed trap concentration.

\subsubsection{Problem 1ja: Radioactive Decay of Mobile Tritium in a Slab (Val-1ja)}

This model is employed to test the first order radioactive decay capabilities of TMAP7. The model assumes pre-charging of a slab with tritium. The tritium was uniformly distributed over the thickness of the slab. The tritium decays to ${ }^{3} \mathrm{He}$ as shown in Equation (1) with a half-life of 12.3232 years.

$$
T \rightarrow{ }^{3} \mathrm{He}
$$

The concentrations of the two species are calculated. The concentration of $\mathrm{T}$ at any given time is given by

$$
C_{t}=C_{t}^{o} \exp (-\omega t)
$$

Applying a mass balance over the system, the concentration of helium is given by

$$
C_{{ }^{3} \mathrm{He}}=C_{t}^{o}[1-\exp (-\omega t)]
$$

where 


$$
\begin{aligned}
C_{t}^{o} & =\text { Initial concentration of tritium } \\
\omega & =\text { rate constant }\left(1.78241 \mathrm{E}-9 \mathrm{~s}^{-1}\right) \\
t & =\text { time }(\mathrm{sec})
\end{aligned}
$$

The comparison between the TMAP7 result and Eqs. (60) and (61) for mobile tritium can be

\begin{tabular}{|c|c|c|c|c|c|c|}
\hline Time (yr) & TMAP7 & [T] Theory & Variance & TMAP7 & [He] Theory & Variance \\
\hline 0.0 & 1.00000 & 1.00000 & 0.00000 & 0.00000 & 0.00000 & 0.00000 \\
\hline 0.4 & 0.97973 & 0.97971 & 0.00002 & 0.02009 & 0.02029 & -0.01001 \\
\hline 0.7 & 0.95987 & 0.95983 & 0.00003 & 0.03997 & 0.04017 & -0.00499 \\
\hline 1.1 & 0.94033 & 0.94036 & -0.00003 & 0.05944 & 0.05964 & -0.00333 \\
\hline 1.5 & 0.92127 & 0.92128 & -0.00001 & 0.07852 & 0.07872 & -0.00254 \\
\hline 1.8 & 0.90260 & 0.90259 & 0.00001 & 0.09722 & 0.09741 & -0.00197 \\
\hline 2.2 & 0.88427 & 0.88428 & -0.00001 & 0.11553 & 0.11572 & -0.00165 \\
\hline 2.6 & 0.86633 & 0.86633 & 0.00000 & 0.13347 & 0.13367 & -0.00144 \\
\hline 2.9 & 0.84880 & 0.84876 & 0.00005 & 0.15105 & 0.15124 & -0.00125 \\
\hline 3.3 & 0.83153 & 0.83154 & 0.00000 & 0.16828 & 0.16846 & -0.00109 \\
\hline 3.6 & 0.81467 & 0.81467 & 0.00000 & 0.18515 & 0.18533 & -0.00098 \\
\hline 4.0 & 0.79813 & 0.79814 & 0.00000 & 0.20168 & 0.20186 & -0.00091 \\
\hline 4.4 & 0.78193 & 0.78194 & -0.00001 & 0.21787 & 0.21806 & -0.00084 \\
\hline 4.7 & 0.76607 & 0.76608 & -0.00002 & 0.23375 & 0.23392 & -0.00075 \\
\hline 5.1 & 0.75053 & 0.75054 & 0.00000 & 0.24929 & 0.24946 & -0.00071 \\
\hline 5.5 & 0.73533 & 0.73531 & 0.00003 & 0.26452 & 0.26469 & -0.00065 \\
\hline 5.8 & 0.72040 & 0.72039 & 0.00001 & 0.27944 & 0.27961 & -0.00061 \\
\hline 6.2 & 0.70580 & 0.70577 & 0.00004 & 0.29406 & 0.29423 & -0.00057 \\
\hline 6.6 & 0.69147 & 0.69145 & 0.00002 & 0.30838 & 0.30855 & -0.00054 \\
\hline 6.9 & 0.67747 & 0.67742 & 0.00006 & 0.32241 & 0.32258 & -0.00052 \\
\hline 7.3 & 0.66371 & 0.66368 & 0.00004 & 0.33615 & 0.33632 & -0.00049 \\
\hline 7.7 & 0.65025 & 0.65022 & 0.00005 & 0.34962 & 0.34978 & -0.00047 \\
\hline 8.0 & 0.63705 & 0.63702 & 0.00005 & 0.36282 & 0.36298 & -0.00043 \\
\hline 8.4 & 0.62413 & 0.62410 & 0.00005 & 0.37575 & 0.37590 & -0.00041 \\
\hline 8.7 & 0.61147 & 0.61144 & 0.00005 & 0.38841 & 0.38856 & -0.00041 \\
\hline 9.1 & 0.59906 & 0.59903 & 0.00005 & 0.40081 & 0.40097 & -0.00039 \\
\hline 9.5 & 0.58691 & 0.58688 & 0.00005 & 0.41297 & 0.41312 & -0.00036 \\
\hline 9.8 & 0.57500 & 0.57497 & 0.00005 & 0.42488 & 0.42503 & -0.00035 \\
\hline 10.2 & 0.56334 & 0.56330 & 0.00006 & 0.43655 & 0.43670 & -0.00034 \\
\hline 10.6 & 0.55191 & 0.55187 & 0.00006 & 0.44798 & 0.44813 & -0.00032 \\
\hline 10.9 & 0.54071 & 0.54068 & 0.00007 & 0.45918 & 0.45932 & -0.00031 \\
\hline 11.3 & 0.52974 & 0.52971 & 0.00006 & 0.47015 & 0.47029 & -0.00031 \\
\hline 11.7 & 0.51899 & 0.51896 & 0.00006 & 0.48090 & 0.48104 & -0.00029 \\
\hline 12.0 & 0.50847 & 0.50843 & 0.00007 & 0.49143 & 0.49157 & -0.00029 \\
\hline 12.4 & 0.49815 & 0.49812 & 0.00007 & 0.50175 & 0.50188 & -0.00027 \\
\hline
\end{tabular}
seen in Table 20. A graphical representation is given in Figure 18.

Table 20. Decay of mobile tritium to $3 \mathrm{He}$ (Val-1ja) 


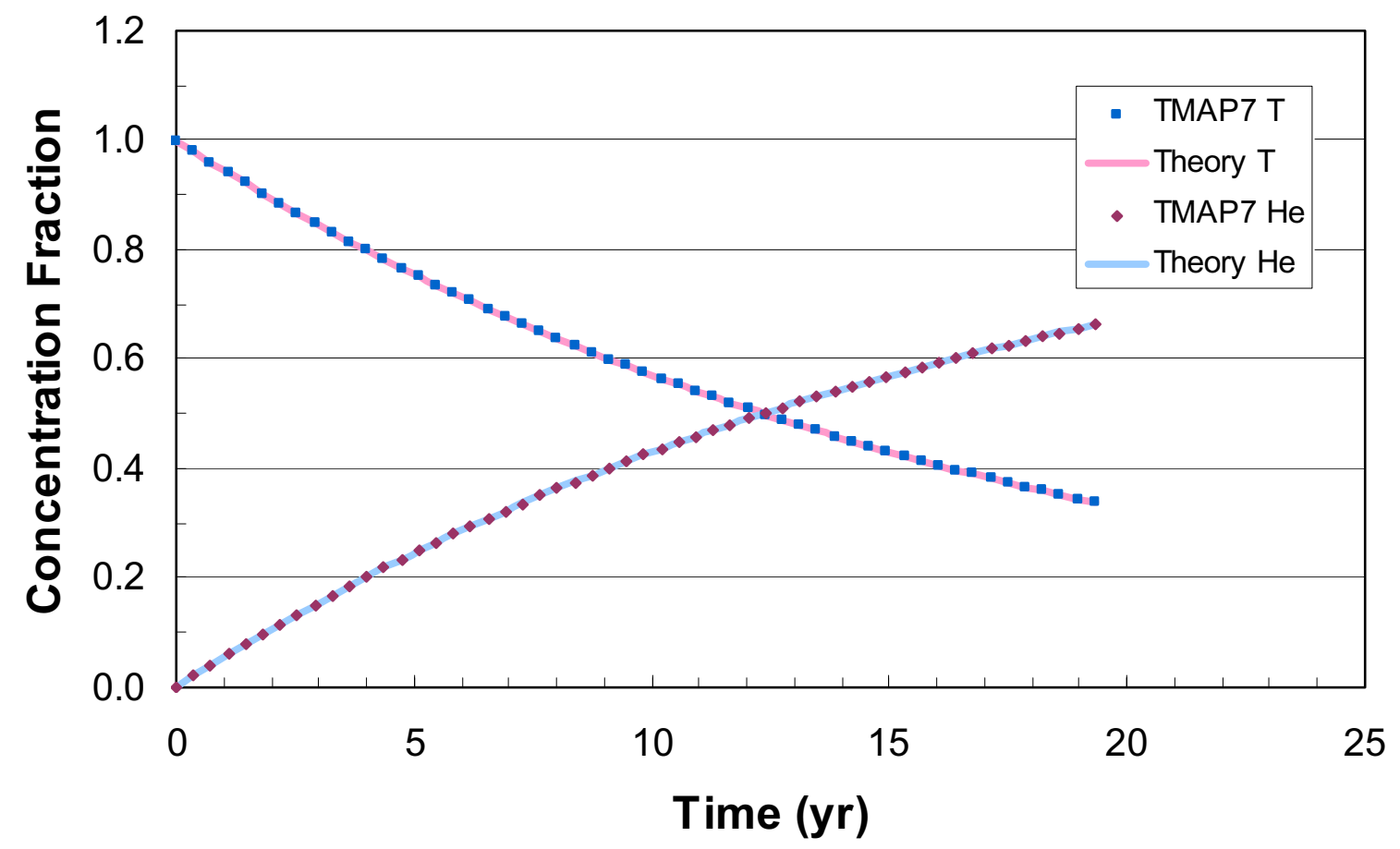

Figure 18. Decay of mobile tritium and associated growth of ${ }^{3} \mathrm{He}$ in a diffusion segment (Val$1 \mathrm{ja})$.

\subsubsection{Problem 1jb: Decay of Tritium in a Distributed Trap (Val-1jb)}

A further but more complex exercise was run for a slab in which nearly all of the tritium is trapped. A slab similar to that used in Problem 1ja was used here, but traps at $1 \%$ atom fraction and $4.2-\mathrm{eV}$ trap energy were distributed in a normal distribution centered at the mid-plane of the slab. The traps were initially filled to $10 \%$ of trap concentration. The mobile atom concentration was only 1 atom $/ \mathrm{m}^{3}$ to begin with, and it very quickly was all absorbed into these deep traps. This problem also demonstrates the utility of the pre-programmed distribution functions for certain parameters.

Figure 19 shows the depth profiles of initial trapped atoms of tritium, final trapped atoms of tritium after 45 years, and the distribution of $\mathrm{He}-3$ at the end of that time. Note that because of finite diffusivity of the $\mathrm{He}-3$, it has broadened a little from the trap concentration. The theoretical solution for this broadening is very complex and is not presented here.

Figure 20 shows the total inventory of tritium in the trap as a function of time over the decay period. It also shows the total helium inventory $\left(\right.$ atoms $\left./ \mathrm{m}^{2}\right)$. The same precision as demonstrated in Problem 1ja was observed here. 


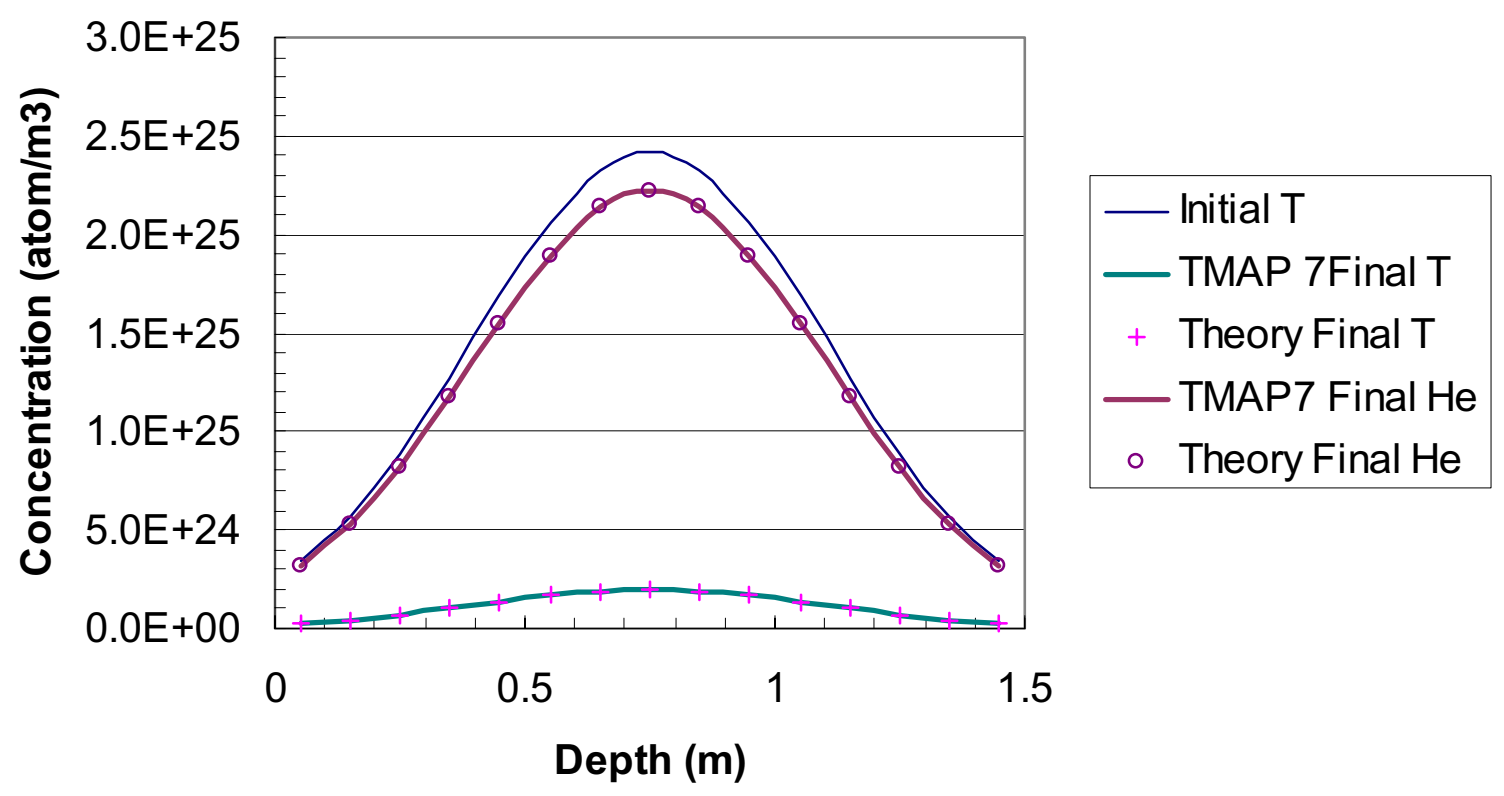

Figure 19. Profiles of trapped tritium at the beginning and end of a 45-year decay and the profile of the resultant ${ }^{3} \mathrm{He}$ at the end of that time (Val-1jb).

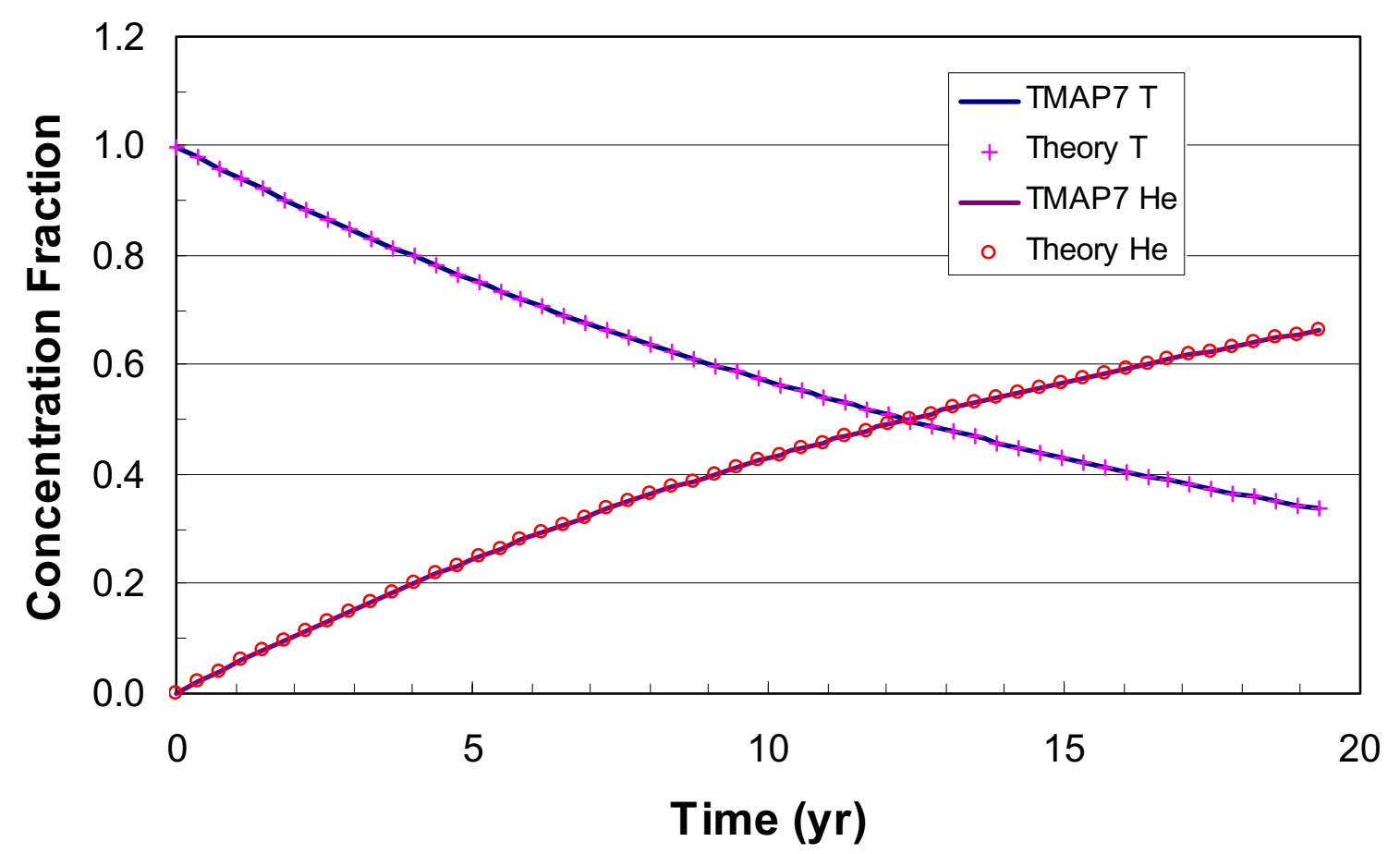

Figure 20. Loss of trapped tritium by radioactive decay is reflected in the gain of He-3 (Val-1jb). 


\subsection{REPLICATING EXPERIMENTS}

The second phase of code validation is the comparison of code results with actual experimental data. Published experiments together with their experimental data were selected for modeling. The first three of these are repeats from the verification and validation of TMAP $4{ }^{6}$

\subsection{Problem 2a: Ion Implantation Experiment (Val-2a)}

This problem is the simulation of experimental results obtained at the INEL in 1985 and published. ${ }^{14}$ The experiment involved applying an ion beam to a 2.5 -cm diameter, $0.5-\mathrm{mm}$ thick sample of a modified 316 stainless steel called Primary Candidate Alloy (PCA). Details of the experiment and the means of evaluating the necessary transport parameters to get a good fit between TMAP7 results and the experimental data are given in the publication. The TRIM code was used to determine that the average implantation depth for the ions was $11-\mu \mathrm{m} \pm 5.4 \mu \mathrm{m}$. Reemission data from the TRIM calculation showed that only $75 \%$ of the incident flux remained in the metal. The other $25 \%$ was re-emitted.

One known non-physical feature in the modeling is that the cleanup of the upstream surface was modeled by a simple exponential in time rather than an ion fluence which was interrupted twice during the actual experiment. The pressures upstream and downstream proved to be inconsequential; they could have been taken as zero and obtained essentially the same results.

The plot of Figure 15 was generated. Actual experimental data are also shown on the figure. They are fairly closely approximated by the calculated permeation. Notice in the figure, however, that in the experimental data there is a lower permeation flux value when the beam is on, and a relatively slow trail-off, compared with the calculation, when the beam was turned off. Some of this is a consequence of the experimental technique where the walls of the experimental chamber did some pumping of the gas as it came through the sample and then provided a source of deuterium when the sample permeation ceased. Some two-dimensional effects also influence the comparison.

Results of this calculation using TMAP7 are essentially identical to those obtained using TMAP4 and reported previously. 


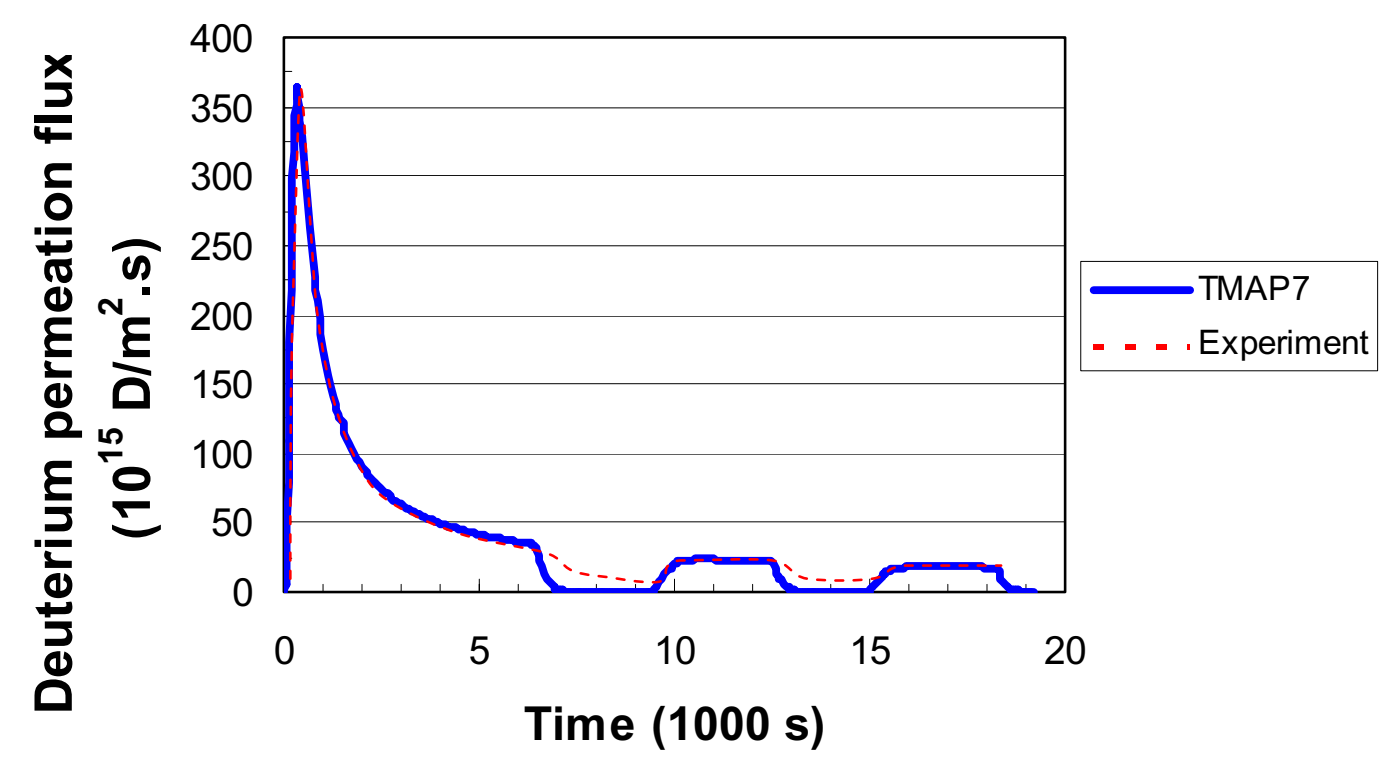

Figure 21. Plasma Driven Permeation of PCA (Val-2a)

\subsection{Problem 2b: Diffusion Experiment in Beryllium (Val-2ba, Val-2bb)}

This problem is taken from work done by R. G. Macaulay-Newcombe at McMaster University. ${ }^{15} \mathrm{He}$ and co-workers conducted thermal absorption and desorption experiments, as well as implantation experiments, on wafers of polished beryllium. Of the several data sets presented, the one modeled here is that represented in Figure 12 (a) in their publication. The beryllium was $0.4-\mathrm{mm}$ thick and had an area of $104 \mathrm{~mm}^{2}$. It was polished to a mirror finish and then exposed to $13.3 \mathrm{kPa}$ of deuterium at $773 \mathrm{~K}$ for 50 minutes. It was quickly cooled under a vacuum of about $1 \mu \mathrm{Pa}$. The cooling time constant for the apparatus is taken as 45 minutes. After removing the sample from the charging furnace, it was transferred in the air to a thermal desorption furnace where the temperature was increased from ambient $(300 \mathrm{~K})$ to $1073 \mathrm{~K}$ at the rate of $3 \mathrm{~K} / \mathrm{min}$. This was done under vacuum, and the pressure of the chamber was monitored by residual gas analysis and calibrated against standard leaks. In that way, the emission rate from the sample could be measured as a function of temperature. Data from that measurement, given in Figure 12 (a) of their paper are reproduced in Figure 22 here. From Rutherford backscattering measurements made on the samples before charging with deuterium, they deduced that the thickness of the oxide film was $18 \mathrm{~nm}$. This is typical for polished beryllium. The metal is so reactive in air that the film forms almost immediately after any surface oxide removal. On the other hand, it is relatively stable and would only grow slightly when exposed to air between charging and thermal desorption.

This experiment is modeled using a two-segment model in TMAP7 with the segments linked. The first is the $\mathrm{BeO}$ film, which is modeled using equally spaced nodes of $1 \mathrm{~nm}$ each plus the two surface nodes. The second segment is a half-thickness wafer of beryllium with reflective boundary conditions at the mid-plane. It is made up of 15 segments of varying thickness to 
accommodate solution stiffness plus the two surface nodes. The solubility of deuterium in beryllium used was that given by K. L. Wilson, et al., ${ }^{16}$ based on work done by W. A. Swansiger, also of Sandia National Laboratory. The diffusivity of deuterium in beryllium was measured by E. Abramov, et. al. ${ }^{17}$. They made measurements on high-grade ( $99 \%$ pure) and extra-grade (99.8\% pure). The values used here are those for high-grade beryllium, consistent with Dr. Macaulay-Newcombe's measurements of the purity of his samples.

Deuterium transport properties of the $\mathrm{BeO}$ are more challenging. First, it is not clear in what state the deuterium exists in the $\mathrm{BeO}$. However, it has been observed ${ }^{18}$ that an activation energy of $-78 \mathrm{Kj}$.mole (exothermic solution) is evident for tritium coming out of neutron irradiated beryllium in work done by D. L. Baldwin of Battelle Pacific Northwest Laboratory. The same energy has appeared in other results (can be inferred from Dr. Swansiger's work cited by Wilson, et al. $^{16}$, and by R. A. Causey, et al. ${ }^{19}$, among others), so one may be justified in using it. The solubility coefficient is not well known. Measurements reported by R. G. Macaulay-Newcombe, et al. ${ }^{20}$ and in follow-up conversations indicate about 200 appm of D in BeO after exposure to $13.3 \mathrm{kPa}$ of $\mathrm{D}_{2}$ at $773 \mathrm{~K}$. That suggests a coefficient of only $1.88 \times 10^{18} \mathrm{~d} / \mathrm{m}^{3} \mathrm{~Pa}^{1 / 2}$. Since much of the deuterium in the oxide layer will get out during the cool-down process (and because it gives a good fit) the solubility coefficient is taken to be $5 \times 10^{20} \mathrm{~d} / \mathrm{m}^{3} / \mathrm{pa}^{1 / 2}$.

Deuterium diffusion measurements in $\mathrm{BeO}$ were made by J. D. Fowler, et al. ${ }^{21}$. They found a wide range of results for diffusivity in $\mathrm{BeO}$, depending on the physical form of the material, having measured it for single-crystal, sintered, and powdered $\mathrm{BeO}$. This model uses one expression for the charging phase and another for the thermal desorption phase, believing that the surface film changed somewhat during the transfer between the two furnaces. For the charging phase diffusivity, the model uses 20 times that for the sintered $\mathrm{BeO}$. Thermal expansion mismatches tend to open up cracks and channels in the oxide layer, so this seems a reasonable value. The same activation energy of $48.5 \mathrm{~kJ} / \mathrm{mole}$, is retained, however. For the thermal desorption phase, the diffusivity prefactor of the sintered material $\left(7 \times 10^{-5} \mathrm{~m}^{2} / \mathrm{sec}\right)$ and an activation energy of $223.7 \mathrm{~kJ} / \mathrm{mole}(53.45 \mathrm{kcal} / \mathrm{mole})$ are used. These values give good results and lie well within the scatter of Fowlers data. Exposure of the sample to air after heating should have made the oxide more like single crystal by healing the cracks that may have developed.

The model applies 13.3. $\mathrm{kPa}$ of $\mathrm{D}^{2}$ for 50 hours followed by evacuation to $1 \mu \mathrm{Pa}$ and cool down with a 45 minute time constant for one hour. The deuterium concentrations in the sample are of a complex distribution that results from first charging the sample and then discharging it during the cool down. This problem is then restarted with different equations to simulate thermal desorption in the $1-\mu \mathrm{Pa}$ environment. That begins at $300 \mathrm{~K}$ and goes to $1073 \mathrm{~K}$. Again, the concentration profiles in both the substrate beryllium and the oxide film have a peculiar interaction because of the activation energies involved, but the flux exuding from the sample when doubled to account for the two sides of the specimen in the laboratory gives a good fit to the experimental data.

From the extracted diffusion species surface flux data for the left side of thermseg/diffseg 1, the solid curve in Figure 22 is constructed where it is compared with the experimental data. Agreement is virtually identical with that found in the TMAP4 calculation for this problem 6 . 


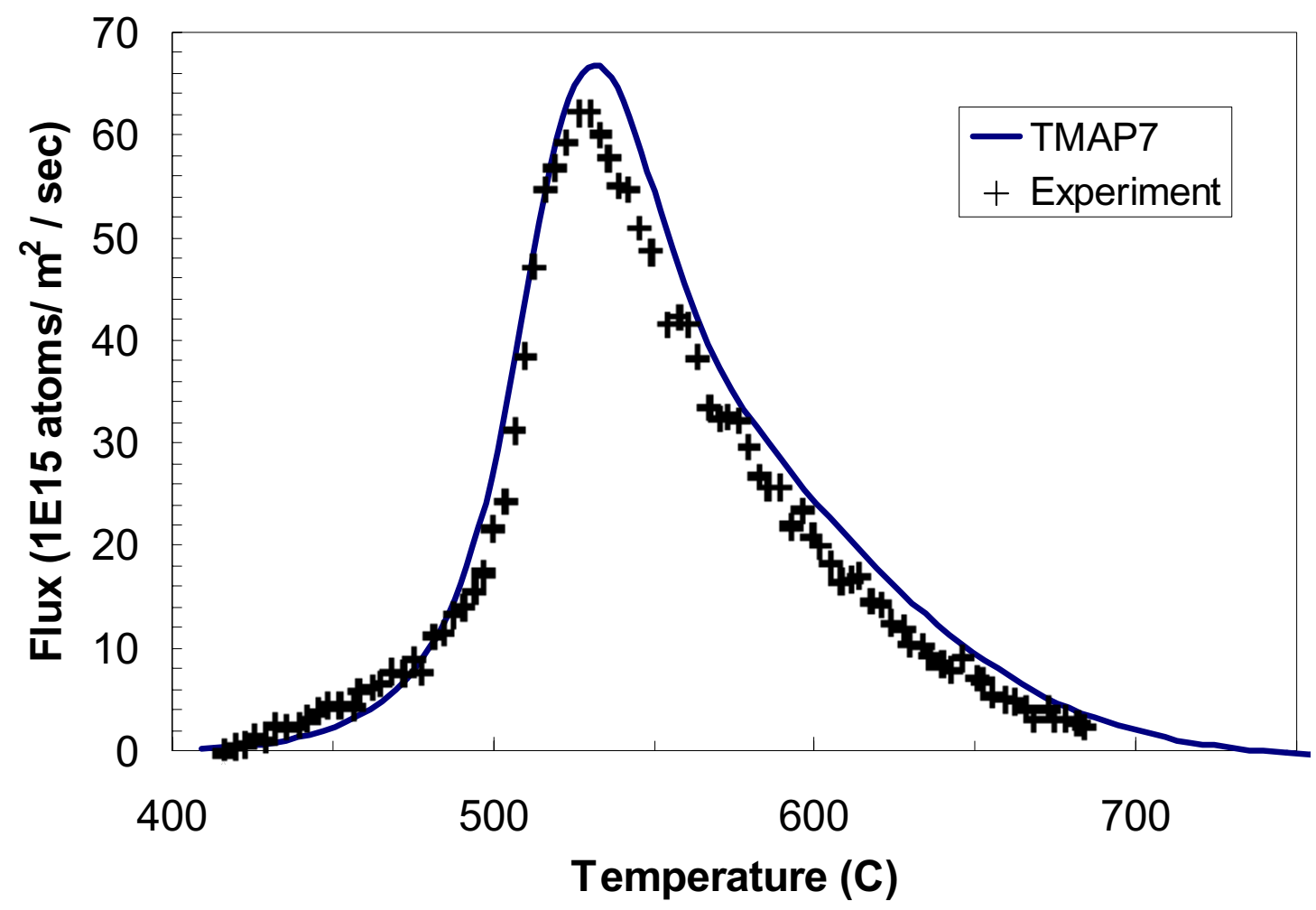

Figure 22. Thermal desorption test of beryllium (Val-2b)

\subsection{Problem 2c: Test Cell Release Experiment (Val-2c)}

This is an experiment that involves multiple enclosures and chemical reactions. It was conducted at the Tritium Systems Test Assembly (TSTA) at Los Alamos National Laboratory and documented by Holland and Jalbert. ${ }^{22}$ The main part of the experiment was an exposure chamber with a nominal volume of $1 \mathrm{~m}^{3}$, which was lined with epoxy paint that is $0.16 \mathrm{~mm}$ thick. Tritium was admitted to the chamber as $\mathrm{T}_{2}$ at the commencement of the experiment. Normally moist (20\% R.H.) air was admitted to the chamber at the rate of $0.54 \mathrm{~m}^{3} / \mathrm{hr}$ constantly throughout the test. Samples of glycol taken form a bubbler just downstream from the exposure chamber were taken at intervals and scintillation counted to determine the time averaged HTO concentration in the chamber as a histogram in time. Tritium and water were absorbed into the paint during the initial part of the test and re-emitted later. Chemical reactions described by the formulae

$$
\begin{aligned}
& \mathrm{T}_{2}+\mathrm{H}_{2} \mathrm{O} \Leftrightarrow H T O+H T \\
& H T+\mathrm{H}_{2} \mathrm{O} \Leftrightarrow H T O+H_{2}
\end{aligned}
$$

took place within the exposure chamber, mainly as a consequence of the radioactivity of the tritium itself. Results of Holland and Jalbert are shown in their Figure 3 from the measurements of the resulting HTO concentration in the exposure chamber following a $10 \mathrm{Ci}$ initial injection (effectively instantaneously) while purging with room air. 
The TMAP7 Model for this experiment consists of three enclosures (1) the room from which air is drawn, (2) the exposure chamber, and (3) the tritium waste treatment system (TWT) to which the exhaust gases are directed. Only enclosure (2) is treated as "functional" or chemically active. The paint on the inside of the exposure chamber is treated as a diffusive segment and non-flow conditions are employed at the interface of the paint with the underlying aluminum foil. Experiments had previous demonstrated that there is virtually no transport of tritium into the aluminum foil. The techniques for determining the constants and other information required to generate a model that gives reasonable results are given by Holland and Jalbert and are not duplicated here.

Data were calculated by TMAP7 for the HTO concentration in the exposure chamber, enclosure 2. A pumping rate of $0.43 \mathrm{~m}^{3} / \mathrm{hr}$ gave a better fit than the apparent one of $0.54 \mathrm{~m}^{3} / \mathrm{hr}$. A solid curve representing these data is compared in Figure 23 with measurements made in bubblers in line with the exposure chamber exhaust. The period over which the bubblers were active in collecting HTO from the exposure chamber is shown on the time scale. They were integrated measurements over the intervals shown. The model fits best at extended times where the intercepts with the "average-value" line segments are at the correct times. Additional uptake and release channels for sort times, beyond those modeled, may be responsible for the early time disparity. A time lag of about 3 hours initially for, say, mixing would make the calculation agree very well with the experiment.

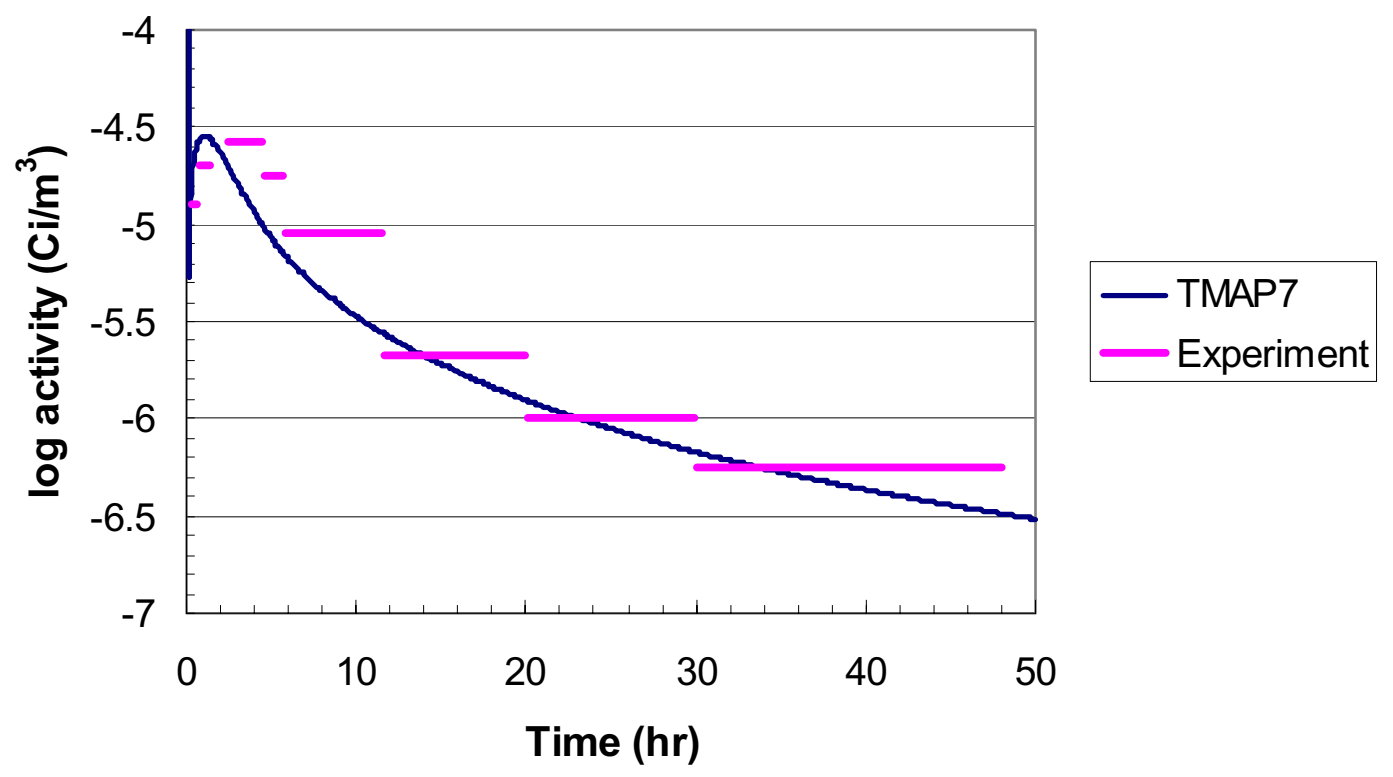

Figure 23. HTO Concentration in TSTA Exposure Chamber (Val-2c).

\subsection{Problem 2d. Thermal Desorption Spectroscopy on Tungsten (Val-2d)}

To exercise surface-law dependent diffusion boundary conditions and at the same time the multiple trapping capability, the experimental result of Hino et al. ${ }^{23}$ was selected for approximation. In this experiment, $\mathrm{H}_{3}^{+}$was implanted at $5 \mathrm{keV}$ and a flux of $1 \times 10^{19} \mathrm{H} / \mathrm{m}^{2} \mathrm{~s}$ for 
5,000 seconds into a polycrystalline tungsten foil $50 \times 50 \mathrm{~mm}^{2}$ and $0.1 \mathrm{~mm}$ thick at room temperature. Background pressure in the implantation chamber was $10^{-3} \mathrm{~Pa}$ while the implantation was going on and $10^{-5} \mathrm{~Pa}$ at other times. Following the implantation, the sample was subjected to thermal desorption spectroscopy by heating under vacuum at $50 \mathrm{~K} / \mathrm{min}$ to $1,273 \mathrm{~K}$ and then held at that temperature for several minutes.

We modeled this system with TMAP7 using the structure of Figure 18. We first supposed that the evacuation pump was rated at $50 \mathrm{~L} / \mathrm{min}$ or $8.33 \times 10^{-4} \mathrm{~m}^{3} / \mathrm{s}$. We then estimated the implantation beam area from the pumping rate, the indicated exposure chamber pressure during implantation of $10^{-3} \mathrm{~Pa}$ of $\mathrm{H}_{2}$, and the implantation flux equivalent of $5 \times 10^{18} \mathrm{H}_{2} / \mathrm{m}^{2} / \mathrm{s}$, which is effectively all re-emitted from the target during irradiation.

$$
A=\frac{P Q}{\phi k T}=\frac{\left(1 \times 10^{-3} \frac{\mathrm{J}}{\mathrm{m}^{3}}\right)\left(8.33 \times 10^{-4} \frac{\mathrm{m}^{3}}{\mathrm{~s}}\right)}{\left(5 \times 10^{18} \frac{\mathrm{H}_{2}}{\mathrm{~m}^{2} \mathrm{~s}}\right)\left(1.3803 \times 10^{-23} \frac{\mathrm{J}}{\mathrm{K}}\right)(300 \mathrm{~K})}=\left(4.025 \times 10^{-5} \mathrm{~m}^{2}\right)
$$

We adjusted the enclosure volume to approximate $t$ the time constant of 625 seconds evident in the decay of what is effectively a pressure measurement at the end of the experiment. The volume chosen was $0.12 \mathrm{~m}^{3}$, which seems reasonable from the sketch provided in the report. ${ }^{23}$

The test chamber was defined for this problem as a functional enclosure. We supposed that at least four pumping time constants (576 s) elapsed after the implantation ended before the thermal ramp began. We assumed the chamber would have a preprogrammed temperature of $300 \mathrm{~K}$ for 7,500 seconds followed by a ramp to $1,273 \mathrm{~K}$ at a ramp rate of $50 \mathrm{~K} / \mathrm{min}$. The vacuum pump is represented by a boundary enclosure (Encl 2) held at $10^{-8} \mathrm{~Pa}$. Gas leakage from the ion source and elsewhere was modeled as a $10^{-5} \mathrm{~Pa}$ boundary enclosure (Encl 3) with flow to the exposure chamber at the vacuum pumping rate. The resulting pressure of background gases in the implantation chamber is thus realistic and reflects the 625-s settling time at the end of the experiment.

On the basis of TRIM $^{2425}$ calculations, implantation was assumed to follow a normal distribution, peaking at $4.6 \mathrm{~nm}$ below the surface and having a scatter or characteristic half width of $3 \mathrm{~nm}$. Implantation was active for 5000 seconds and then terminated.

The diffusion boundary condition employed was the surfdep or surface law dependent with the following parameter values

\section{atomic hydrogen, $\mathbf{H}$}

$$
\begin{aligned}
& v=\text { DeBye frequency, } 8.4 \mathrm{E}-12\left(\mathrm{~s}^{-1}\right) \\
& E_{c}=\text { surface binding energy, }-0.8(\mathrm{eV}) \\
& E_{S}=\text { solution enthalpy, } 1.04(\mathrm{eV}) \\
& P_{c}=\text { combination probability, } 1.0\left(\text { to form } \mathrm{H}_{2}\right)
\end{aligned}
$$




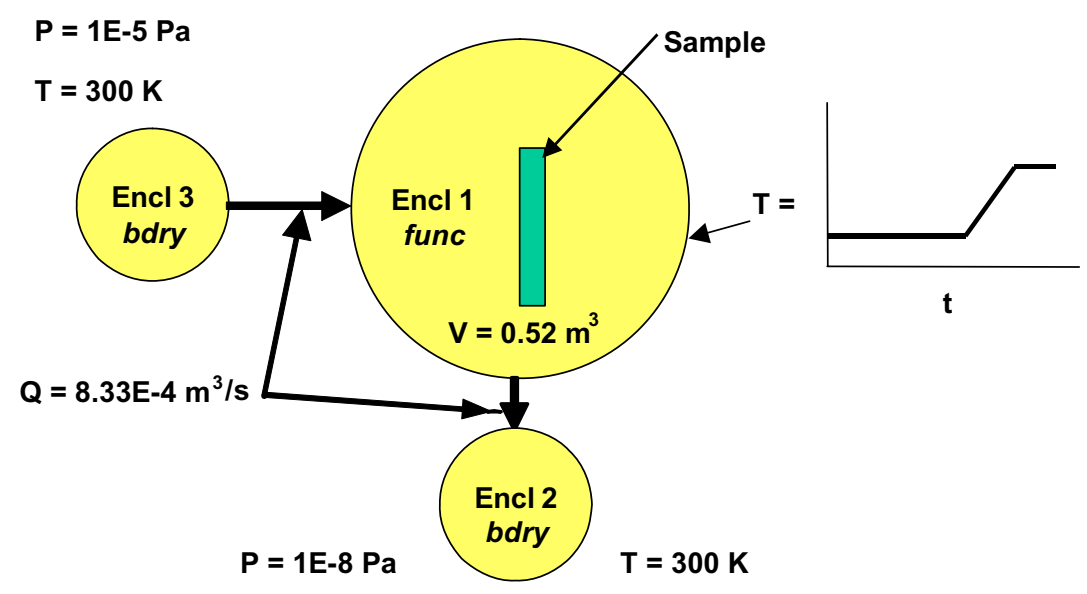

Figure 24. Schematic of system used to model experiments of Hino et al. ${ }^{23}$

\section{surface hydrogen, $\mathrm{H}_{2}$}

$v_{\mathrm{o}}=$ DeBye frequency, 8.4E-12 $\left(\mathrm{s}^{-1}\right)$

$E_{c}=$ surface binding energy, $-0.1(\mathrm{eV})$

$E_{x}=$ surface barrier energy, $0.05(\mathrm{eV})$

$E_{b}=$ dissociation energy, $0.05(\mathrm{eV})$

$M_{m}=$ molecular mass, $2.0(\mathrm{amu})$

$P_{c}=$ formation probability, 1.0 (when $\mathrm{H}$ finds $\mathrm{H}$ )

$v_{s}=$ stoichiometric exponent, 0.5 (molecules per $\mathrm{H}$ combined)

For solubility of $\mathrm{H}$ in $\mathrm{W}$, we use the value given by Frauenfelder. ${ }^{26}$

$$
S=1.83 \times 10^{24}\left(\frac{H}{m^{3}}\right) \exp \left(-\frac{1.04 \mathrm{eV}}{R T}\right)
$$

Diffusivity used for $\mathrm{H}$ through $\mathrm{W}$ was the normally accepted Frauenfelder value. ${ }^{26}$

$$
D=4.1 \times 10^{-7}\left(\frac{\mathrm{m}^{2}}{\mathrm{~s}}\right) \exp \left(-\frac{0.39 \mathrm{eV}}{R T}\right)
$$

$\mathrm{H}_{2}$ was considered insoluble in $\mathrm{W}$ and therefore had no diffusivity through the bulk. However, the surface diffusivity was taken to be

$$
D=4.1 \times 10^{-7}\left(\frac{\mathrm{m}^{2}}{\mathrm{~s}}\right) \exp \left(-\frac{0.1 \mathrm{eV}}{R T}\right)
$$


Three traps were assumed in the sample. Trap concentrations and distributions were considered adjustable parameters while energies were determined by TDS peak temperatures. The first was assumed to be associated with implantation (damage and precipitation) and to be normally distributed with a peak at $4.6 \mathrm{~nm}$ and a characteristic width of $10 \mathrm{~nm}$, consistent with the observations of Haasz et al. ${ }^{27}$ that damage zone exceeds the implantation depth. Its trap energy was adjusted, based on the temperature of the first peak, to be $1.0 \mathrm{eV}$, and it was assumed to be $7 \%$ atom fraction and uniform throughout the implantation region. A slightly lower value, say $6 \%$ or $5.5 \%$ would have given a better fit with the measured data. The second was a uniform trap, probably associated with dislocations and was assigned a trap release energy of $1.35 \mathrm{eV}$, typical of but slightly higher than that seen by Anderl et al ${ }^{28}$ Its concentration was adjusted to $1.38 \mathrm{E}-05$ atom fraction. The third trap was also assumed to be uniformly distributed and to have a trapping energy of $2.7 \mathrm{eV}$, nearly the same as the deep trap seen by Frauenfleder ${ }^{26}$ with a concentration of 5.9E- 06 atom fraction. These were assumed to be approximately $90 \%$ filled at the start of the experiment, the hydrogen having come from air water vapor following previous anneals. Assuming emptier traps results in somewhat lower emissions, especially for the intermediate energy trap, because they do not completely fill in the 5,000 seconds of implantation. Higher diffusivity would correct that.

Even though the measured flux in the experiment was given in terms of a molecular flux density from the surface of the sample, it is likely that this corresponded to a pressure in the vicinity of the sample. Hence, the results reported here are those calculated for the enclosure $\mathrm{H}_{2}$ pressure. The implantation flux of $10^{19} \mathrm{H} / \mathrm{m}^{2} / \mathrm{s}$ generated a molecular gas $\left(\mathrm{H}_{2}\right)$ pressure of $1 \times 10^{-3} \mathrm{~Pa}$ during implantation. Therefore, a peak emission rate of $10^{18} \mathrm{H}_{2} / \mathrm{m}^{2} / \mathrm{s}$ during thermal desorption would correspond to a molecular gas pressure of $1 \times 10^{-4} \mathrm{~Pa}$. The computed results are shown together with the scaled Hino data in Figure 19.

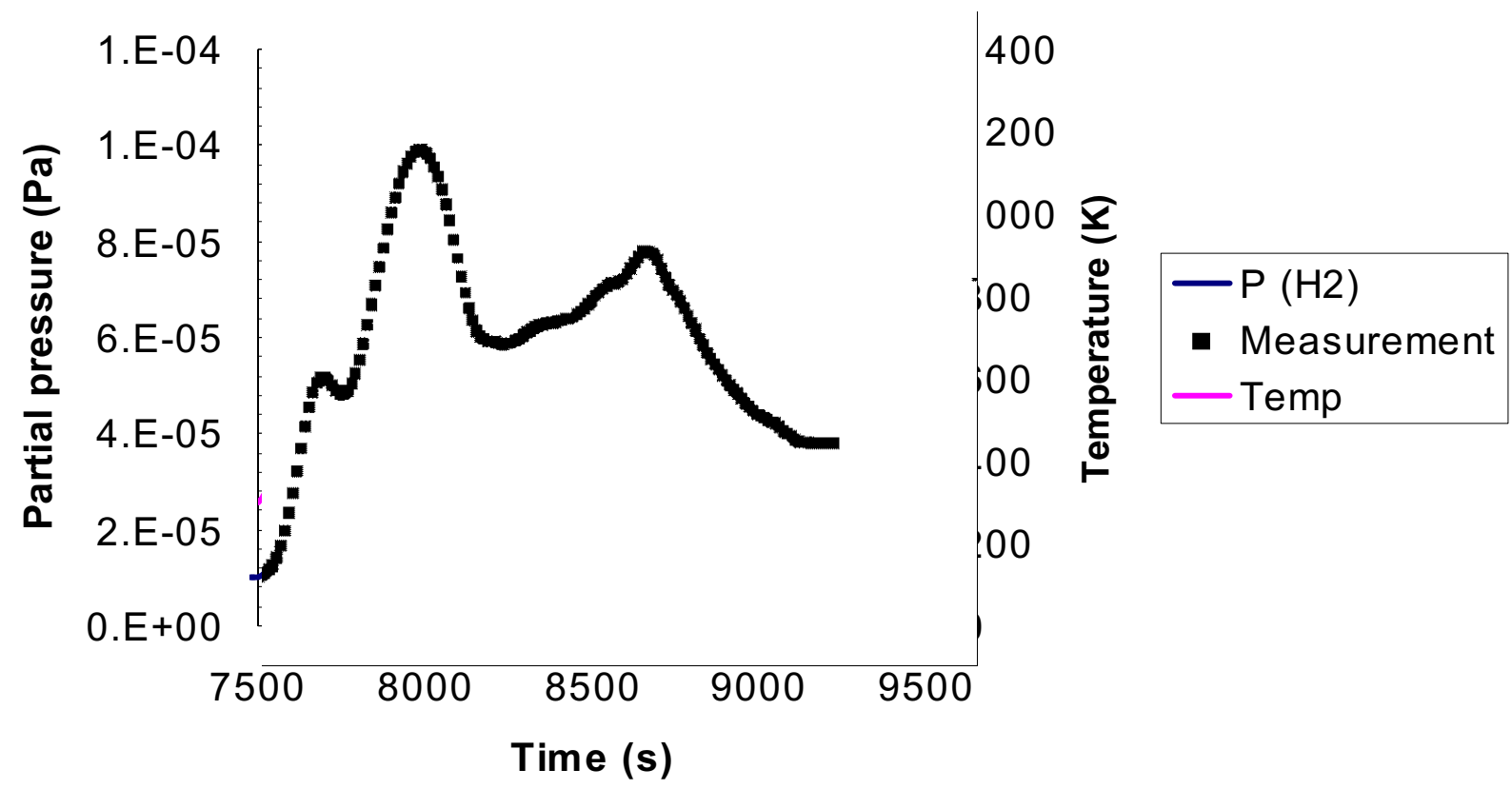

Figure 25. Comparison of calculated with experimental results for Hino's experiment with implantation and thermal desorption of tungsten (Val-2d). 
The fit with the Hino et al. data is not exact because of several factors, the most prominent of which is probably that there are more than three traps. It appears that there may be several between 1.35 and $2.7 \mathrm{eV}$.

\subsection{Problem 2e. Co-permeation of $\mathrm{H}_{2}$ and $\mathrm{D}_{2}$ through Pd (Val-2e)}

This problem was selected to demonstrate a non-classical solution law boundary condition with molecular exchange as well as combined solution-law and recombination limited boundary conditions. It comes from work reported by Kizu et al. ${ }^{29}$ on experiments in which $\mathrm{H}_{2}$ and $\mathrm{D}_{2}$ were allowed to permeate through thin Pd membranes either separately or together. The tests resulted in the formation of $\mathrm{HD}$, both on the upstream side and on the downstream side of the membrane.

The experimental apparatus consisted of two vacuum chambers separated by a Pd membrane which was $1.8 \times 10^{-4} \mathrm{~m}^{2}$ in area and either $0.025 \mathrm{~mm}$ or $0.05 \mathrm{~mm}$ thick, depending on the test. The membrane was clamped on each side by a copper gasket, and it may reasonably be inferred that the only means of transfer of gas from one chamber to the other was by diffusion through the membrane. Temperatures in the membrane were controlled between 820 and $870 \mathrm{~K}$ by means of an electric resistance heater surrounding the membrane and a thermocouple touching the membrane. Gas was introduced into one of the chambers from regulated supply bottles at various compositions and pressures. Here, we refer to that chamber as the upstream chamber. The base pressure on both upstream and downstream chambers was maintained at less than $10^{-6} \mathrm{~Pa}$ by a combination of turbomolecular pump and rotary backing pump on each side. Pressure was indicated by an ion gage on each side, and gas composition was measured with a quadrupole mass spectrometer. Flow rates through the membrane were determined by pressure increases in the downstream chamber at fixed pumping rate of $0.1 \mathrm{~m}^{3} / \mathrm{s}$.

The first tests reported were permeation tests of $\mathrm{D}_{2}$ alone through membranes of each thickness. For the thinner membrane, tests were conducted at both $825 \mathrm{~K}$ and $865 \mathrm{~K}$ whereas the $0.05-\mathrm{mm}$ membrane was tested only at $825 \mathrm{~K}$. These were performed to calibrate the permeability of the membranes to hydrogen isotopes. Figure 20 shows their experimental data for permeation flux, $\mathrm{J}\left(\mathrm{D}_{2}\right)$, as a function of upstream $\mathrm{D}_{2}$ pressure, $\mathrm{P}\left(\mathrm{D}_{2}\right)$.

Also shown in Figure 26 are three "fit" lines. Kizu et al. observed that at low pressures the permeation flux is directly proportional to the upstream gas pressure. As pressure increases, the permeation flux falls off from that linear relationship and approaches a square root relationship. Here, the fit to the $0.05-\mathrm{mm}$ data $(825 \mathrm{~K})$ is made using least-squares methods across the range of pressures measured, not just at the lower pressures where greater linearity is observed. The fit line to the $0.025-\mathrm{mm}$ data $(825 \mathrm{~K})$ is not really a fit at all. It is simply the line from the $0.05-\mathrm{mm}$ data multiplied by a factor of 2 . It fits the data amazingly well, indicating that permeation through the membrane is diffusion-limited, not surface-limited. The fit line for the $865-\mathrm{K}$ data, also an extrapolation, has the same slope (0.8958) as the previous two fit lines, but it is offset by a factor of 1.55 from the $0.05-\mathrm{mm}(825 \mathrm{~K})$ line. It does not fit the higher-pressure data as well as it does the low-pressure data, but it does suggest a permeability activation energy of $0.674 \mathrm{eV}$ $(7,818 \mathrm{~K})$. The resulting equation for $\mathrm{D}_{2}$ permeability in $\mathrm{Pd}$ is thus 


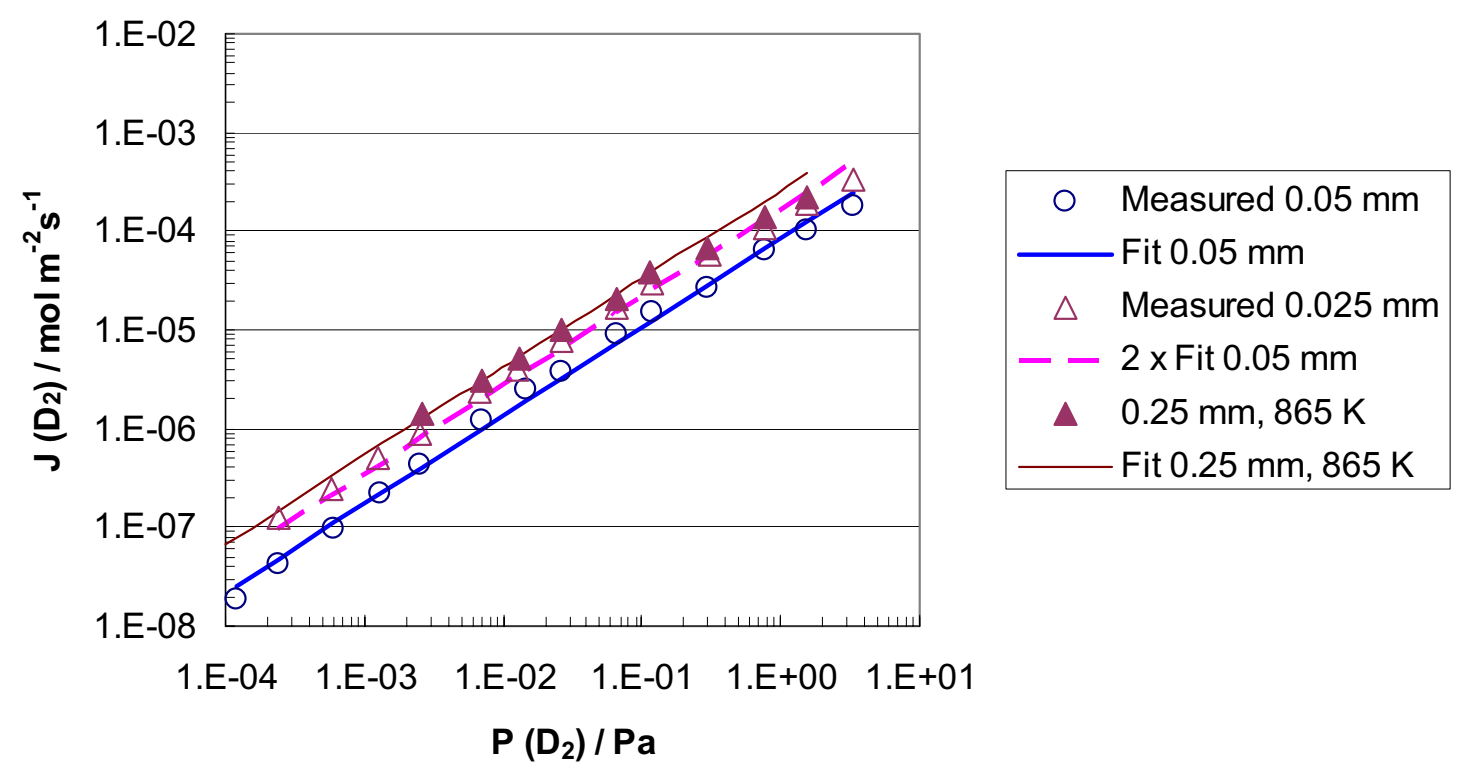

Figure 26. Permeability data of Kizu et al. for $\mathrm{D}_{2}$ in Pd.

$$
J=\frac{1.096 \times 10^{-4}}{L} P^{0.8958} \exp \left(-\frac{7818}{T}\right)\left(\frac{m o l e}{m^{2} s}\right)
$$

where

$$
\begin{aligned}
& L=\text { membrane thickness }(\mathrm{m}) \\
& P=\text { upstream pressure }(\mathrm{Pa}) \\
& T=\text { Temperature }(\mathrm{K})
\end{aligned}
$$

For the diffusion-limited regime, permeability is the product of solubility, $S$, and diffusivity, $D$, such that, approximately

$$
J=\frac{C_{0}}{L} D=\frac{S P^{v}}{L} D=\frac{S_{0} P^{v} D_{0}}{L} \exp \left[-\frac{\left(E_{d}+E_{s}\right)}{k T}\right]
$$

where $E_{d}$ and $E_{s}$ are the diffusion activation energy and solution enthalpy, respectively. Comparing Eqs. (67) and (68), we see that

$$
\begin{aligned}
& v=0.8958 \\
& S_{0} D_{0}=1.096 \times 10^{-4} \\
& E_{d}+E_{s}=7,818 k
\end{aligned}
$$

We can separate diffusivity and solubility by making use of the diffusivity of hydrogen in $\mathrm{Pd}$ given by Katz and Gulbransen ${ }^{30}$ divided by $\sqrt{2}$ to account for isotopic effect on diffusivity

$$
D_{D}=3.048 \times 10^{-7} \exp \left(-\frac{2818}{T}\right)\left(\frac{m^{2}}{s}\right)
$$


That leaves for deuterium

$$
S_{D}=179.6 \exp \left(-\frac{5000}{T}\right)\left(\frac{\text { mole }}{m^{3} P a^{v}}\right)=1.082 \times 10^{26} \exp \left(-\frac{5000}{T}\right)\left(\frac{\text { atom }}{m^{3} \mathrm{~Pa}^{v}}\right)
$$

Kizu et al. ${ }^{29}$ note that the solubility of hydrogen in Pd is about 1.23 times as great as the solubility of deuterium in $\mathrm{Pd}$ at the temperature of their experiments.

Next, we construct a model for TMAP7 simulation of this experiment. We consider two functional enclosures, each with an estimated volume of $0.1 \mathrm{~m}^{3}$, separated by a diffusion segment of thickness $L$ and area $1.8 \times 10^{-4} \mathrm{~m}^{2}$. This is illustrated in Figure 27 .

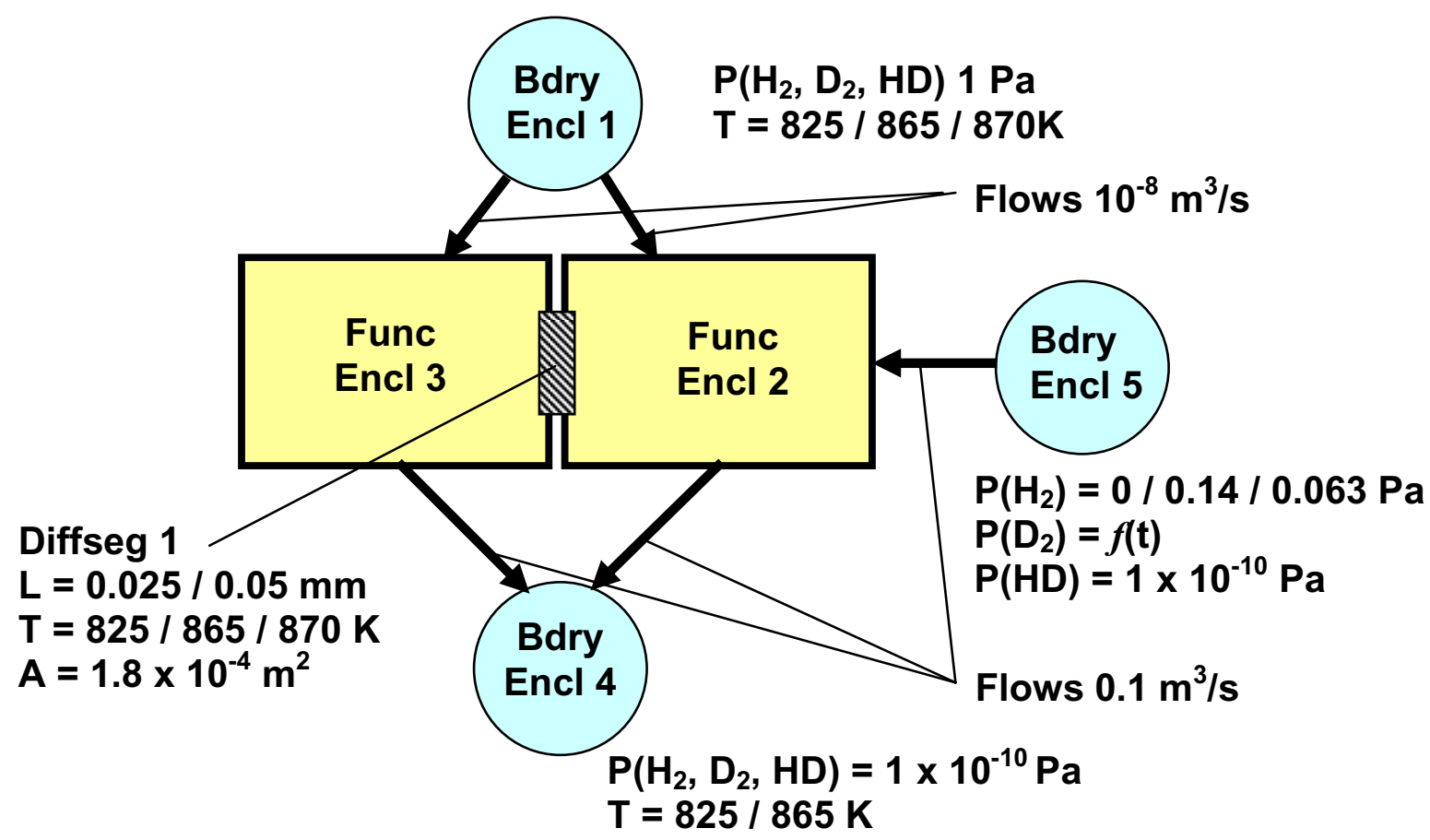

Figure 27. TMAP7 model of experimental system of Kizu et al.

Boundary enclosure 1 is the source of background pressure to the experimental system. Boundary enclosure 4 is the vacuum pumping system that provides a sink for all system flows. Boundary enclosure 5 is the gas feed to the upstream experimental chamber, functional enclosure 2. Depending on the experiment, the feed pressure of $\mathrm{H}_{2}$ is $0,0.14 \mathrm{~Pa}$, or $0.063 \mathrm{~Pa}$. Combined with the evacuation to boundary enclosure 4 , this provides the upstream $\mathrm{H}_{2}$ pressure for permeation. The $\mathrm{D}_{2}$ pressure is a stepped function of time, one step corresponding to each of the data points in the data plots of Kizu et al. Steps are arbitrarily set at $100 \mathrm{~s}$, but equilibrium is achieved in times much shorter than that. No HD is fed into the upstream experimental chamber, in keeping with the experimental setup given by Kizu et al. For the solution-law (lawdep) boundary conditions, it is assumed that HD is formed in accordance with the laws of chemical equilibrium.

$$
P_{H D}=2 \sqrt{P_{H_{2}} P_{D_{2}}}
$$


Under these conditions, one specifies the homonuclear gas pressures, and TMAP7 determines what the corresponding heteronuclear gas pressure must be. Thus, for this analysis, one specifies effective deuterium pressure $P_{D}$, where

$$
P_{D}=P_{D_{2}}+\frac{P_{H D}}{2}
$$

and similarly for $P_{H}$.

In the experiments, even though $\mathrm{H}_{2}$ gas flow is fixed, it is not $P_{H_{2}}$ that is fixed but $P_{H}$, because as the $\mathrm{D}_{2}$ pressure increases, some of the $\mathrm{H}_{2}$ will be converted to HD. Under equilibrium (lawdep) conditions, it may be shown that

$$
P_{H D}=\frac{2 P_{H} P_{D}}{P_{H}+P_{D}}
$$

which, with Eq. (73) leads to

$$
P_{D_{2}}=\frac{P_{D}^{2}}{P_{H}+P_{D}}
$$

Likewise, for the calculation, we will not have constant $P_{\mathrm{H}_{2}}$, but $P_{\mathrm{H}_{2}}$ will vary according to

$$
P_{H_{2}}=\frac{P_{H}^{2}}{P_{H}+P_{D}}
$$

For the non-equilibrium (ratedep) boundary condition, the pressure in enclosure 5 will be the specified $P_{D}$, and the code will determine the enclosure pressures of $\mathrm{H}_{2}, \mathrm{D}_{2}$, and $\mathrm{HD}$ from the pumping, dissociation, and recombination rates.

For the downstream chamber, functional enclosure 3 under lawdep conditions, HD is formed together with $\mathrm{H}_{2}$ and $\mathrm{D}_{2}$ in chemical equilibrium from diffusing $\mathrm{H}$ and $\mathrm{D}$. Under ratedep conditions, again the relative dissociation and recombination rates together with the convective flows determine the partial pressures in enclosure 3.

We first replicate the calibration experiments shown in Figure 26 using input files Val-2ea.inp, Val-2eb.inp, and Val-2ec.inp for the three cases shown in Figure 26. Results are in Figure 28. The results are almost as good as the approximations for the permeability in Figure 26. 


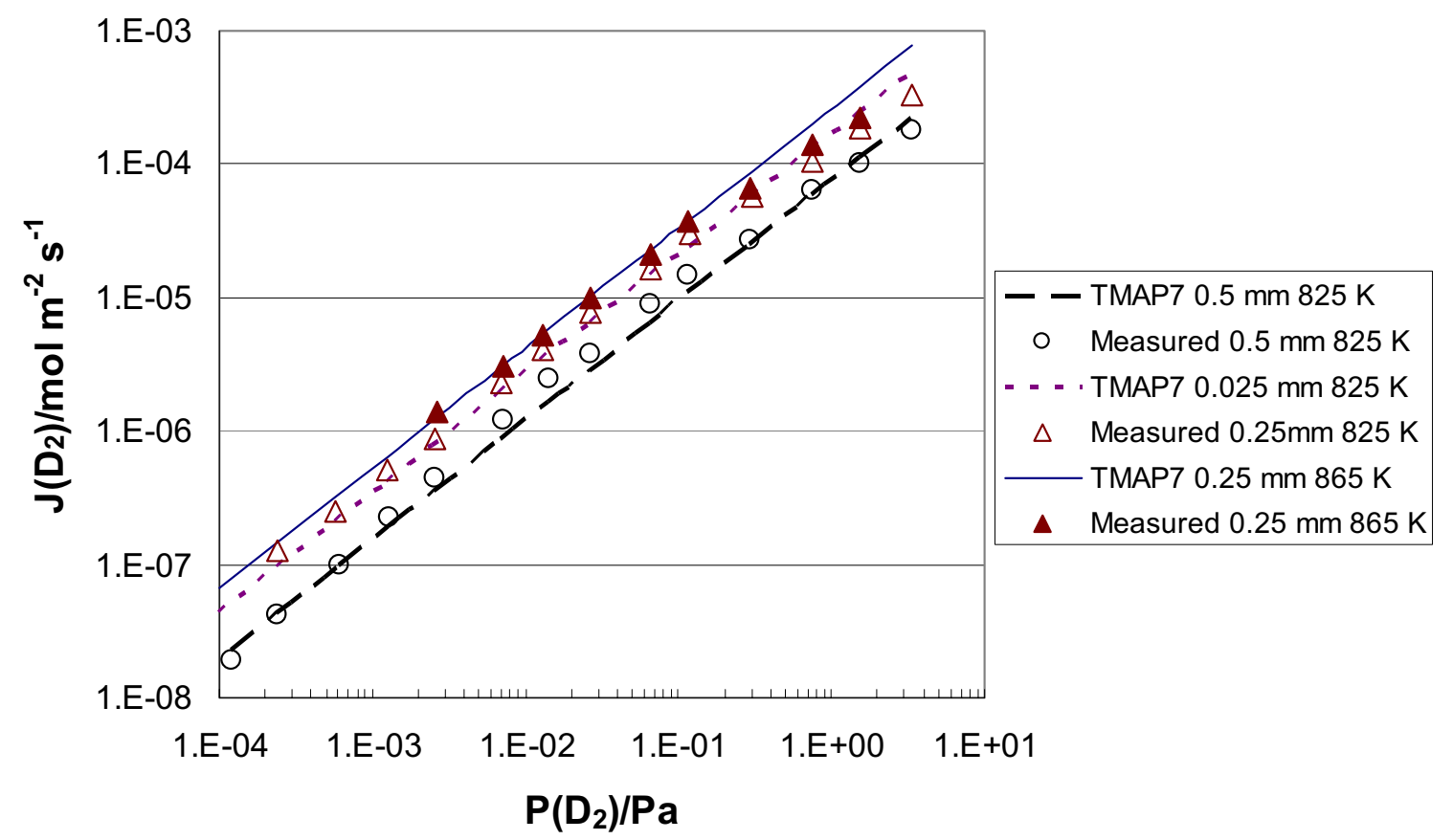

Figure 28. Comparison of TMAP7 permeation calculations with permeation data of Kizu et al. for $\mathrm{D}_{2}$ only under lawdep boundary conditions using the solubility of Eq. (71) (Val-2ea, Val-2eb, Val-2ec).

In modeling the co-permeation of $\mathrm{H}$ and $\mathrm{D}$, we first apply the lawdep boundary condition in which we apply $\mathrm{H}_{2}$ through enclosure 5 at pressures indicated by Eq. (76) for pre-selected values of $P_{H}(0.063 \mathrm{~Pa})$ and $P_{D}$ corresponding to the abscissa values of the data in Kizu et al. ${ }^{29} \mathrm{D}_{2}$ is also added at pressures given by Eq. (75) for the same $P_{H}$ and set of $P_{D}$ values for the experiment on a $0.025-\mathrm{mm}$ membrane at $870 \mathrm{~K}(\mathrm{Val}-2$ ed.inp $)$. The results of that computation are compared with the experimental data in Figure 29. 


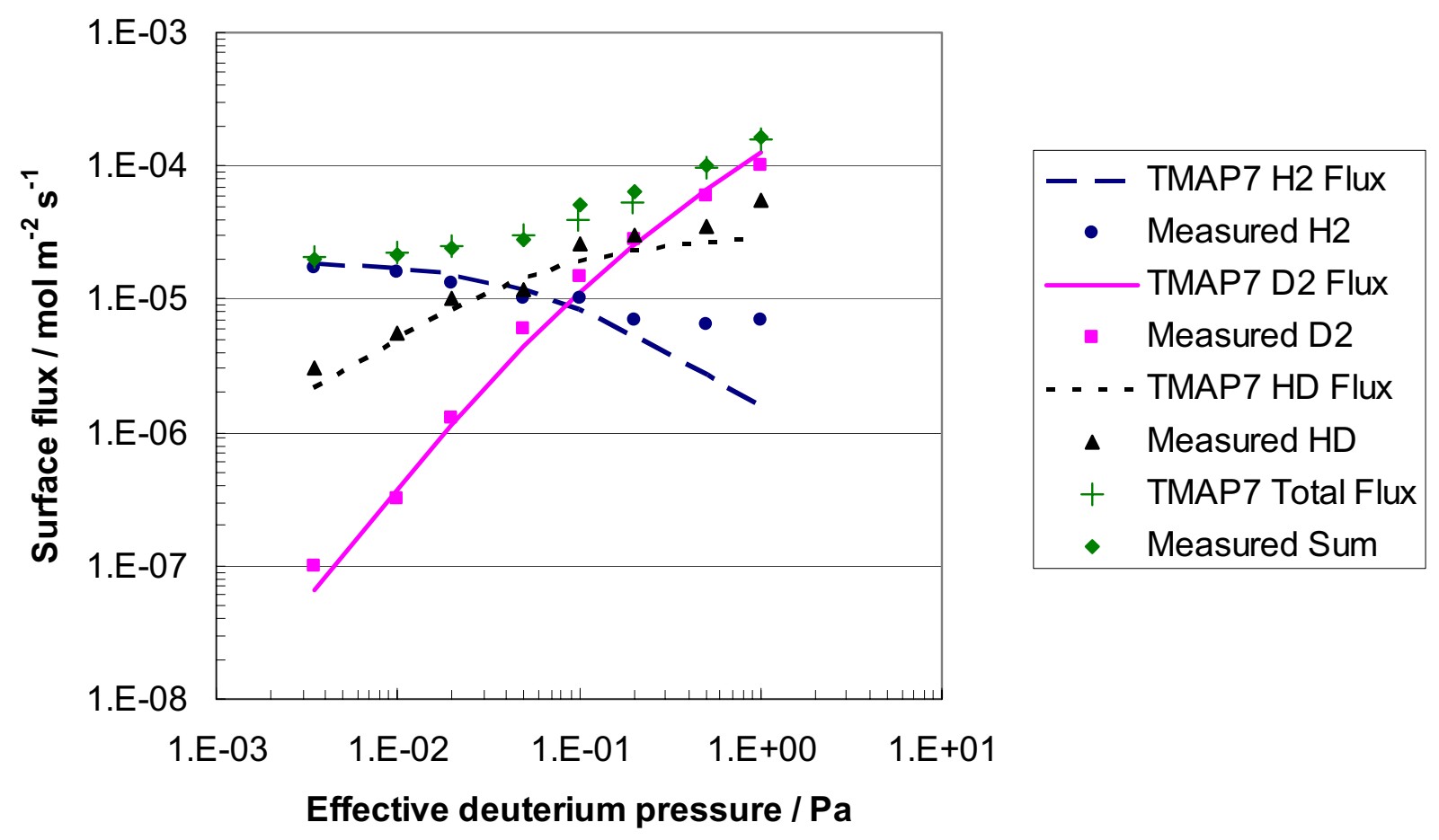

Figure 29. Comparison of TMAP7 results using a lawdep boundary condition on each side of the membrane wirh the experiment s of Kizu et al. (Val-2ed).

It is evident that while the $\mathrm{H}_{2}$ permeation calculated at low effective deuterium pressures agree well with the experimental data, they do not agree at higher deuterium pressures. Likewise, $\mathrm{HD}$ release rates are under-predicted at high $\mathrm{D}_{2}$ pressures. $\mathrm{D}_{2}$ release rates agree reasonably well at higher and lower pressures but slightly less well at intermediate pressures.

For additional perspective, we next changed the diffusion boundary condition to the ratedep mode in which dissociation and recombination take place independently (Val-2ee.inp). We use for the dissociation rate coefficient the molecular arrival rate at the surface

$$
K_{d}=\frac{1}{\sqrt{2 \pi \mathrm{M} k T}}=\frac{2.6276 \times 10^{24}}{\sqrt{M T}}\left(\frac{\text { molecule }}{m^{2} P a}\right)
$$

where $M$ is the species molecular weight in amu. For the recombination coefficient, we use the relationship from Sieverts' law that

$$
K_{r}=\frac{K_{d}}{S^{2}}
$$

For deuterium, using $S$ from Eq. (71),

$$
K_{r_{D 2}}=\frac{1.994 \times 10^{-28}}{\sqrt{M T}} \exp \left(\frac{10000}{T}\right)\left(\frac{m^{4}}{s}\right)
$$

while for hydrogen with its higher solubility 


$$
K_{r_{H 2}}=\frac{1.318 \times 10^{-28}}{\sqrt{M T}} \exp \left(\frac{10000}{T}\right) \quad\left(\frac{m^{4}}{s}\right)
$$

For HD we use the average of these two. The results from that computation are as shown in Figure 30.

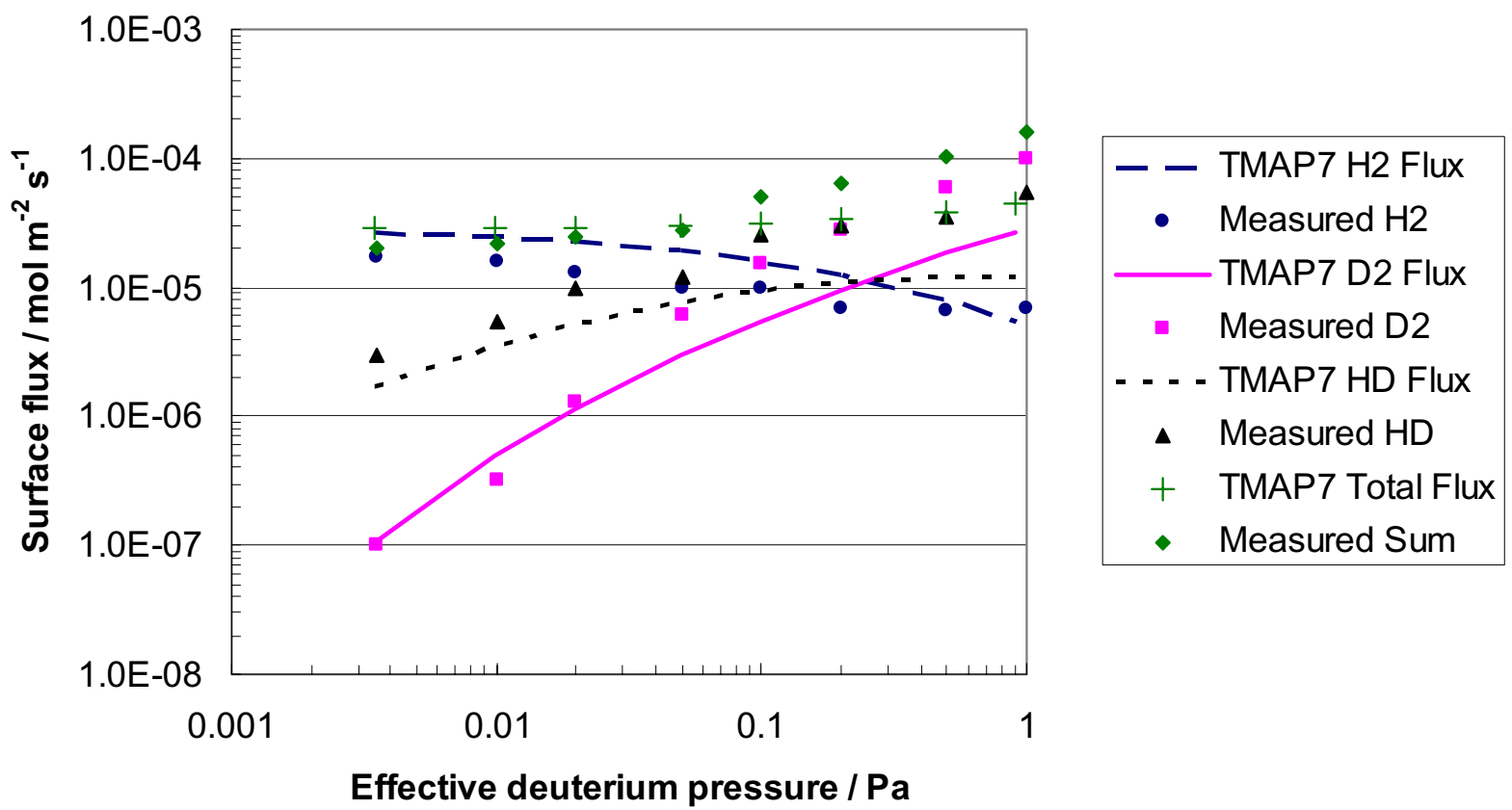

Figure 30. Comparison of TMAP7 calculation with simple ratedep boundary conditions with the values measured by Kizu et al. (Val-2eea).

Here, $\mathrm{H}_{2}$ permeation at low $\mathrm{D}_{2}$ pressures is over-predicted but it improves at higher deuterium pressures. Agreement for HD is better at low pressures than high. $\mathrm{D}_{2}$ permeation is good at low $\mathrm{D}_{2}$ pressures but it under-predicts markedly at high pressures. Total permeation rate is good at intermediate pressures but it is over-predicted at low pressures and under-predicted at high pressures. It appears that more deuterium and hydrogen are getting into the upstream face of the membrane at higher pressures than are predicted by the model. Increasing $K_{d}$ to the full arrival rate has little effect on results. Reducing $K_{d}$ to 0.1 of the molecular arrival rate and using the same approach shifts the permeation curves upward, as shown in Figure 31. 

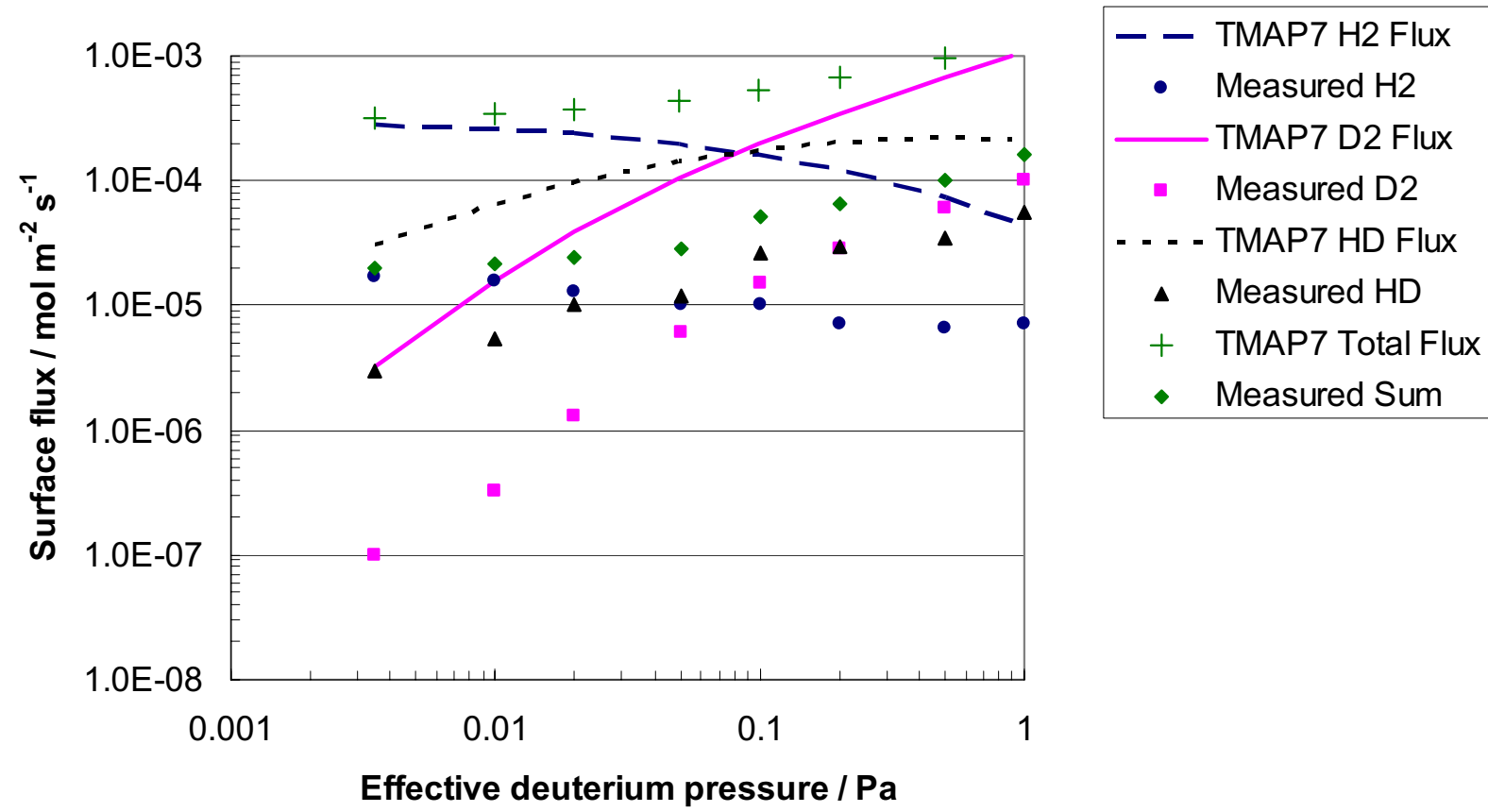

Figure 31. TMAP7 results from a 5-fold reduction in $K_{d}$ and $K_{r}$ (Val-2eeb).

Though the fit is not as good as the lawdep solution, these results are consistent with the observations of Kizu et al. that permeation appears to be nearly first-order in $\mathrm{P}$ at low pressures but tends to become proportional to $P^{1 / 2}$ as driving pressure increases. For a variation, the problem was rerun with a lawdep upstream diffusion boundary condition and a ratedep boundary condition downstream (Val-2ef). The results are shown in Figure 32. The fit is not particularly good anywhere.

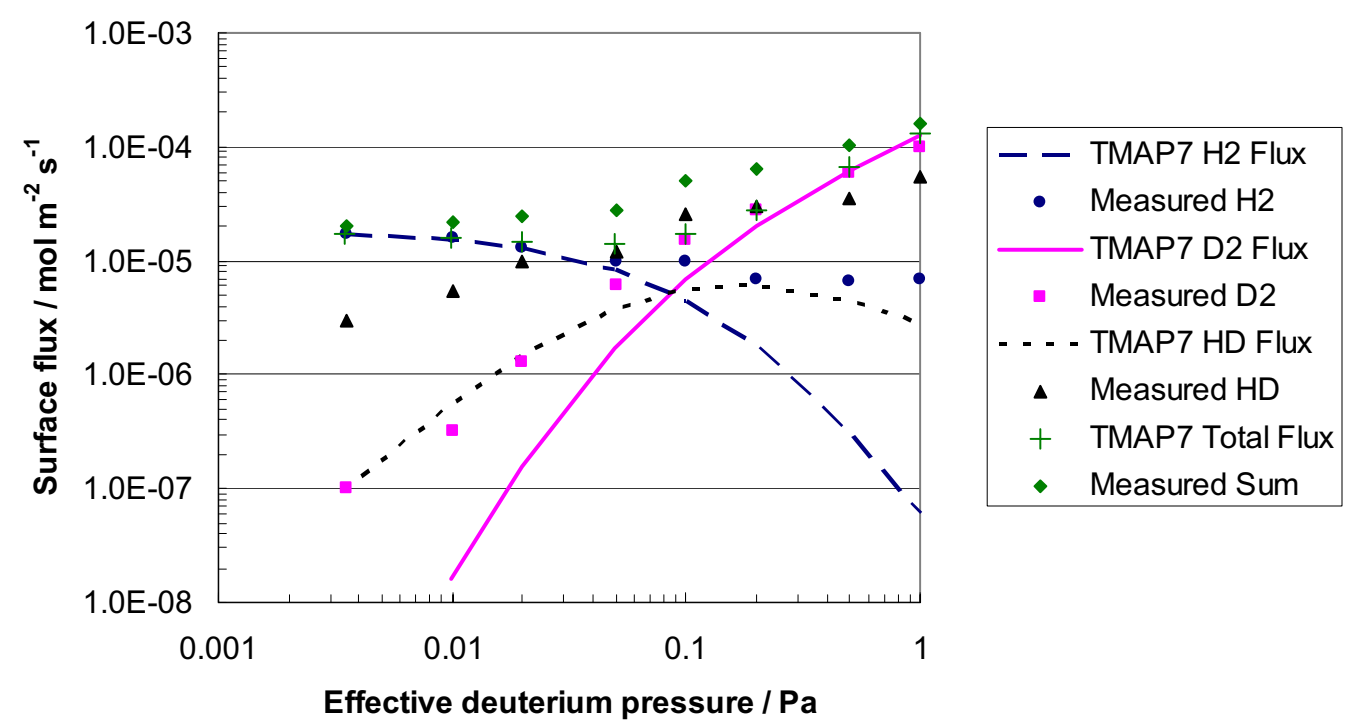

Figure 32. Comparison of TMAP7 calculation for lawdep boundary condition upstream and ratedep boundary condition downstream with measurements made by Kizu et al. (Val-2ef). 


\subsection{CONCLUSIONS}

In the course of the work performed here, the TMAP7 code has been demonstrated in a wide variety of applications. Many of these are contrived problems for which analytical solutions are available. Agreement between solutions calculated by TMAP7 and those generated in a Microsoft Excel ${ }^{\mathrm{TM}}$ spreadsheet is excellent. A second group of problems constitute replications of actual experiments, the results of which appear in published journals. By making use of accepted values of transport parameters and some fitting constant values, it has been shown that TMAP7 gives results in good agreement with actual measurements. These two groups of exercises constitute the verification and validation of the TMAP7 code.

The major challenge in assembling the computational models is finding the necessary parameters for the various property values needed in the code. A further challenge with TMAP7 is one faced by many such codes, numerical convergence. This is managed with various control parameters to adjust the damping in convergence iteration.

TMAP7 represents a significant step forward in modeling gas interaction with structures and in enclosures. 


\section{REFERENCES}

1. B. J. Merrill, et al., April 11, 1998, TMAP/MOD1, Tritium Migration Analysis Program Code Description and User's Manual, EGG-EP-7407, Idaho National Engineering and Environmental Laboratory, Idaho Falls, Idaho.

2. G. R. Longhurst, May 2000, TMAP2000 Development, INEEL/EXT-2000-678, Idaho National Engineering and Environmental Laboratory, Idaho Falls, Idaho.

3. H. S. Carslaw and J. C. Jaeger, "Conduction in Heat and Solids $2^{\text {nd }}$ Edition, Oxford University Press, 1959, p. 128.

4. H. S. Carslaw and J. C. Jaeger, "Conduction in Heat and Solids $2^{\text {nd }}$ Edition, Oxford University Press, 1959, p. 60.

5. D. Zwillinger, "CRC Standard Mathematical Tables and Formulae $30^{\text {th }}$ Edition", CRC Press, 1996

6. G. R.Longhurst et al., "Verification and Validation of TMAP4," EGG-FSP-10347, Rev.01, March 19, 1994, Idaho National Engineering Laboratory.

7. H. S. Carslaw and J. C. Jaeger, "Conduction in Heat and Solids $2^{\text {nd }}$ Edition, Oxford University Press, 1959, p. 62.

8. G. R. Longhurst, "Trapping Effects on Diffusion Transients", INEL, EG\&G Idaho, Inc., May 2, 1990

9. H. S. Fogler, "Elements of Chemical Reaction Engineering $3^{\text {rd }}$ Edition", Prentice Hall PTR, 1999

10. F. P. Incropera and, D. P. DeWitt, "Fundamentals of Heat and Mass Transfer", New York, John Wiley \& Sons, 2002

11. Hibbitt, Karlsson, \& Sorensen, Inc., ABAQUS/Standard, Version 6.3-1, Pawtuckett, Rhode Island.

12. H. B. Dwight, 1961, Tables of Integrals and Other Mathematical Data, $4^{\text {th }}$ Ed., New York, The Macmillan Company, p. 29.

13. H. S. Fogler, 1999, "Elements of Chemical Reaction Engineering $3^{\text {rd }}$ Edition", Upper Saddle River, NJ, Prentice Hall PTR.

14. R. A. Anderl, et al., "Tritium Permeation in Stainless Steel Structures Exposed to Plasma Ions," Proceedings, Eleventh Symposium on Fusion Engineering, November 18-22, 1985, Ausin, TX, Vol I, pp. 644-649, IEEE Cat. No. CH2251-7.

15. R. G. Mccaulay-Newcombe et al., "Deuterium Diffusion, Trapping and Release in Ionimplanted Beryllium," Fusion Engineering and Design 8 (1991) 419.

16. K.L. Wilson, et al., "Beryllium--a better tokamak plasma-facing material?" Journal of Bacuum Science and Technology_A 8 (3) (May/June-1990) 1750.

17. E. Abramov, et al.,"Ceuterium Permeation and Diffusion in High Purity Beryllium," CFFTP-G-9013, May 1990.

18. G. R. Longhurst, "Tritium Behavior in ITER Beryllium," EGG-FSP-9034, October 1990. 
19. R. A. Causey, et al., "Tritium rentention and migration in beryllium," Journal of Nuclear Materials, 176 \& 177 (1990) 654.

20. R. G. Macaulay-Newcombe, et al., "Thermal Absorption and Desorption of Deuterium in Beryllium and Beryllium Oxids," Fifth Inernational Conference on Fusion Reactor Materials, Clearwater, FL, November 20-24, 1991

21. J. D. Fowler, et al., "Tritium Diffusion in $\mathrm{Al}_{2} \mathrm{O}_{3}$ and $\mathrm{BeO}$, 'Journal of the American Ceramic Society", 60 (3-4) (Mar/Apr 1977) 155.

22. D. F. Holland and R. A. Jalbert, "A Model for Tritium Concentration Following Tritium Release into a Test Cell and Subsequent Operation of an Atmospheric Cleanup Systen," Proceedings, Eleventh Symposium of Fusion Engineering, Novermber 18-22, 1985,. Austin, TX, Vol I, pp. 638-43, IEEE Cat. No. CH2251-7.

23. T. Hino et al., "Hydrogen retention properties of polycrystalline tungsten and helium irradiated tungsten," Fusion Engineering and Design, 39-40 (1998) 227-233.

24. W. Eckstein, 1991, Computer Simulation of Ion-Solid Interactions, New York, SpringerVerlag.

25. J. F. Ziegler, et al., 1985, The Stopping and Range of Ions in Solids, New York, Pergamon Press.

26. R. Frauenfelder, "Solution and Diffusion of Hydrogen in Tungsten," Journal of Vacuum Science and Technology, 6 (1969) 388-397.

27. A. A. Haasz et al., "The effect of ion damage on deuterium trapping in tungsten," Journal of Nuclear Materials, 266-269 (1999) 520-525.

28. R. A. Anderl et al., "Hydrogen Transport and Trapping in Polycrystalline Tungsten," Fusion Technology, 21 (1992) 745-750.

29. K. Kizu, A. Pisarev, and T.Tanabe, "Co-permeation of deuterium and hydrogen through Pd,” Journal of Nuclear Materials, 289 (2001) 291-302.

30. O.M. Katz and E. A. Gulbransen, Review of Scientific Instruments, 31 (1960) 615-617. 


\section{APPENDIX A \\ SPECIES EQUILIBRATION MODEL}

Suppose that two homonuclear diatomic molecular species, $\mathrm{A}_{2}$ and $\mathrm{B}_{2}$, are in a volume $V$, and at time $t=0$, are allowed to contact a catalytic surface of area $S$ that supports the reaction

$$
\frac{1}{2} A_{2}+\frac{1}{2} B_{2} \leftrightarrow A B
$$

Assume further that the molecular species have the same mass and chemical properties such that there is no enthalpy change associated with this reaction and only configurational entropy is driving the reaction. Then

$$
\Delta G_{f}=-T \Delta s_{f}=-R T \ln 2
$$

The equilibrium constant for reaction (A-1) is then

$$
K_{e q}=\exp \left(-\frac{\Delta G_{f}}{R T}\right)=2
$$

The law of mass action then requires that in equilibrium,

$$
\frac{[A B]}{\left[A_{2}\right]^{\frac{1}{2}}\left[B_{1}\right]^{\frac{1}{2}}}=2
$$

or equivalently

$$
P_{A B}=2 P_{A_{2}}^{\frac{1}{2}} P_{B_{2}}^{\frac{1}{2}}
$$

The $A B$ molecules come from the dissociation of $A_{2}$ and $B_{2}$ molecules such that for starting pressures $P_{A_{2}}^{0}$ and $P_{B_{2}}^{0}$, it must also be true that at equilibrium

$$
P_{A B}=2 \frac{P_{A_{2}}^{0} P_{B_{2}}^{0}}{P_{A_{2}}^{0}+P_{B_{2}}^{0}}
$$

Two different approaches to the dynamics of the equilibration process will now be explored, one corresponding to ratedep boundary conditions and the other to surfdep conditions.

\section{Ratedep Conditions}

At equilibrium, when Sieverts' law applies, for atom concentrations $C_{A}$ and $C_{B}$ at the surface,

$$
\begin{aligned}
& C_{A}=K_{s} \sqrt{P_{A_{2}}} \\
& C_{B}=K_{s} \sqrt{P_{B_{2}}}
\end{aligned}
$$

where $K_{s}$ is the Sieverts' solubility. Because of the assumed equality of chemistry, $K_{S}$ will be the same for each homonuclear species. We expect also that under equilibrium conditions 


$$
K_{d} P_{A_{2}}=K_{r} C_{A}^{2}
$$

where $K_{d}$ is the dissociation coefficient and $K_{r}$ is the recombination coefficient. That leads to

$$
K_{d}=K_{s}^{2} K_{r}
$$

We expect further for the heteronuclear species

$$
K_{d} P_{A B}=K_{r_{A B}} C_{A} C_{B}
$$

Under ratedep conditions, equilibrium is not assumed, but the relationships between the coefficients are maintained. Under these assumed conditions, the dissociation coefficients for both $A B$ and $A_{2}$ or $B_{2}$ molecules should be identical. However, because two different microscopic processes can produce $A B$ ( $A$ jumping to find $B$ and $B$ jumping to find $A$ ) and only one ( $A$ finding $A$ ) can form $A_{2}$, and similarly for $B_{2}$, we expect $K_{r_{A B}}$ to be twice $K_{r}$ for the homonuclear molecules.

We first write conservation equations for the surface species, $C_{A}$ and $C_{B}$.

$$
\begin{aligned}
& C_{A}\left(C_{A}+C_{B}\right) 2 K_{r}=K_{d}\left(2 P_{A_{2}}+P_{A B}\right) \\
& C_{B}\left(C_{A}+C_{B}\right) 2 K_{r}=K_{d}\left(2 P_{B_{2}}+P_{A B}\right)
\end{aligned}
$$

Adding these together and applying the conservation of gas atoms in the enclosure gives

$$
\left(C_{A}+C_{B}\right)^{2}=K_{s}^{2}\left(P_{A_{2}}^{0}+P_{B_{2}}^{0}\right)
$$

This requires that $C_{A}$ and $C_{B}$ are both constant.

The current of $A B$ molecules from surface $S$ from volume $V$ is the rate of change of those molecules in the enclosure.

$$
\frac{d N_{A B}}{d t}=S\left(2 K_{r} C_{A} C_{B}-K_{d} P_{A B}\right)
$$

Here, $N_{A B}$ is the number of molecules of species $A B$ in the enclosure. Solving Equations (A-11) for $C_{A} C_{B}$, we find that

$$
C_{A} C_{B}=\frac{K_{d} P_{A_{2}}^{0} P_{B_{2}}^{0}}{2 K_{r}\left(P_{A_{2}}^{0}+P_{B_{2}}^{0}\right)}
$$

Then, Equation (A-13) becomes

$$
\frac{d P_{A B}}{d t}=\frac{S K_{d} k T}{V}\left(\frac{2 P_{A_{2}}^{0} P_{B_{2}}^{0}}{\left(P_{A_{2}}^{0}+P_{B_{2}}^{0}\right)}-P_{A B}\right)
$$

Equation (A-15) is solved by

$$
P_{A B}=\frac{2 P_{A_{2}}^{0} P_{B_{2}}^{0}}{\left(P_{A_{2}}^{0}+P_{B_{2}}^{0}\right)}\left[1-\exp \left(-\frac{S K_{d} k T}{V} t\right)\right]
$$




\section{Surfdep Conditions}

When surfdep conditions apply, there are no assumptions about equilibrium except in the steady state. Then, the surface concentration of molecules is directly proportional to the gas overpressure and we define a deposition rate constant by.

$$
\hat{K}_{d}=\frac{1}{\sqrt{2 \pi M k T}} \exp \left(-\frac{E_{x}}{k T}\right)
$$

where $M$ is the mass of any of the species molecules, assuming all are equal, and $E_{x}$ is the adsorption barrier energy. For release of the molecular species from the surface,

$$
\hat{K}_{r}=\frac{v_{o}}{6} \exp \left(\frac{E_{c}-E_{x}}{k T}\right)
$$

Here, $v_{o}$ is the Debye frequency, $E_{c}$ is the surface binding energy, and the factor of 6 accounts for the probability that a given phonon will be directed away from the surface. At steady-state, the flux to the surface will be balanced by flux from the surface, and surface concentration will be related to the gas over-pressure by

$$
C_{m_{s}}=P_{m} \frac{\hat{K}_{d}}{\hat{K}_{r}}=\frac{6 P_{m}}{v_{o} \sqrt{2 \pi M k T}} \exp \left(-\frac{E_{c}}{k T}\right)
$$

The conversion of $A_{2}$ and $B_{2}$ molecules to $A B$ molecules requires several steps. First, homonuclear molecules in the gas must get to the surface. Next, they must dissociate. Then the individual surface atoms must migrate to sites where they encounter their conjugates. Here we assume there is a probability of unity of their combination once they find each other. Finally, the $A B$ molecule must leave the surface and return to the gas. We write equations for species continuity at the surface.

$$
\begin{aligned}
& C_{A B}\left(\hat{K}_{r}+\hat{K}_{b}\right)=P_{A B} \hat{K}_{d}+C_{A} C_{B}\left(2 D_{s} \lambda\right) \\
& C_{A_{2}}\left(\hat{K}_{r}+\hat{K}_{b}\right)=P_{A_{2}} \hat{K}_{d}+C_{A}^{2}\left(D_{s} \lambda\right) \\
& C_{B_{2}}\left(\hat{K}_{r}+\hat{K}_{b}\right)=P_{B_{2}} \hat{K}_{d}+C_{B}^{2}\left(D_{s} \lambda\right) \\
& C_{A}\left[\left(C_{A}+C_{B}\right) 2 D_{s} \lambda\right]=\left(C_{A B}+2 C_{A_{2}}\right) K_{b} \\
& C_{B}\left[\left(C_{A}+C_{B}\right) 2 D_{s} \lambda\right]=\left(C_{A B}+2 C_{B_{2}}\right) K_{b}
\end{aligned}
$$

In these equations, the dissociation rate for molecules at the surface is given by

$$
K_{b}=\exp \left(-\frac{E_{b}}{k T}\right)
$$

where $E_{b}$ is the dissociation activation energy, $D_{s}$ is the surface diffusivity of the atomic species, and $\lambda$ is the lattice constant, assumed to be the reciprocal cube root of the lattice density. $K_{b}$ is assumed equal for all molecular species, and $D_{s}$ is assumed to be the same for all atomic species.

We may combine Equations (A-17) to (A-21) to find that 


$$
P_{\text {total }}=P_{A_{2}}+P_{B_{2}}+P_{A B}=\left(C_{A}+C_{B}\right)^{2}\left(\frac{\hat{K}_{r}}{K_{b}}\right) \frac{D \lambda}{\hat{K}_{d}}
$$

This is reminiscent of Sieverts' law. With the conservation law for atoms in the gas

$$
P_{A B}=2\left(P_{A_{2}}^{0}-P_{A_{2}}\right)=2\left(P_{B_{2}}^{0}-P_{B_{2}}\right)
$$

Equation (A-23) becomes

$$
\left(C_{A}+C_{B}\right)^{2}=\left(P_{A_{2}}^{0}+P_{B_{2}}^{0}\right) \frac{\hat{K}_{d}}{D_{s} \lambda} \frac{K_{b}}{\hat{K}_{r}}
$$

Note that no assumption has been made regarding steady state. Because the sum of the concentrations $C_{A}$ and $C_{B}$ is constant in time for this problem, either the individual concentrations must both be constant or a change in one must be the negative of a change in the other. The latter case is not consistent with the definition of present problem. Therefore, they must both be constant. Then, from statistical considerations, the molecular formation rates must be the same as they are in steady state.

The process that converts dissociation products to $A B$ molecules is the recombination step while the net destruction rate is dissociation. Hence

$$
\frac{d N_{A B}}{d t}=S\left(C_{A} C_{B} 2 D_{s} \lambda-C_{A B} K_{b}\right)
$$

Equation (A-17) must hold at all times such that if we solve it for $C_{A B}$ and substitute the result into Equation (A-26) we get, successively

$$
\begin{aligned}
& \frac{d N_{A B}}{d t}=S\left(C_{A} C_{B} 2 D_{s} \lambda-K_{b} \frac{P_{A B} \hat{K}_{d}+C_{A} C_{B} 2 D_{s} \lambda}{\hat{K}_{r}+K_{b}}\right) \\
& \frac{d P_{A B}}{d t}=\frac{S k T}{V}\left[C_{A} C_{B} 2 D_{s} \lambda\left(1-\frac{K_{b}}{\hat{K}_{r}+K_{b}}\right)-P_{A B} \frac{\hat{K}_{d} K_{b}}{\hat{K}_{r}+K_{b}}\right] \\
& \frac{d P_{A B}}{d t}=\frac{S k T}{V} \frac{\hat{K}_{d} K_{b}}{\hat{K}_{r}+K_{b}}\left[C_{A} C_{B} 2 D \lambda\left(\frac{\hat{K}_{r}}{\hat{K}_{d} K_{b}}\right)-P_{A B}\right]
\end{aligned}
$$

This is solved by the expression

$$
P_{A B}=C_{A} C_{B} 2 D \lambda \frac{\hat{K}_{r}}{\hat{K}_{d} K_{b}}\left[1-\exp \left(-\frac{S k T t}{V} \frac{\hat{K}_{d} K_{b}}{\hat{K}_{r}+K_{b}}\right)\right]
$$

It may be shown, again using Equations (A-17) to (A-21), that this is equivalent to

$$
P_{A B}=2 \frac{P_{A_{2}}^{0} P_{B_{2}}^{0}}{P_{A_{2}}^{0}+P_{B_{2}}^{0}}\left[1-\exp \left(-\frac{t}{\tau}\right)\right]
$$

where 


$$
\tau=\frac{V\left(\hat{K}_{r}+K_{b}\right)}{S k T \hat{K}_{d} K_{b}}
$$

A - 5 


\section{APPENDIX B PROBLEM INPUT FILE LISTINGS}

In this appendix are the input file listings used in the demonstration problems in Sections 2 and 3. These may be used as starting points for individual problems by the user. 


$$
\text { B - } 2
$$


Problem 1a: Diffusion from a Depleting Source (Val-1a, see p. 2)

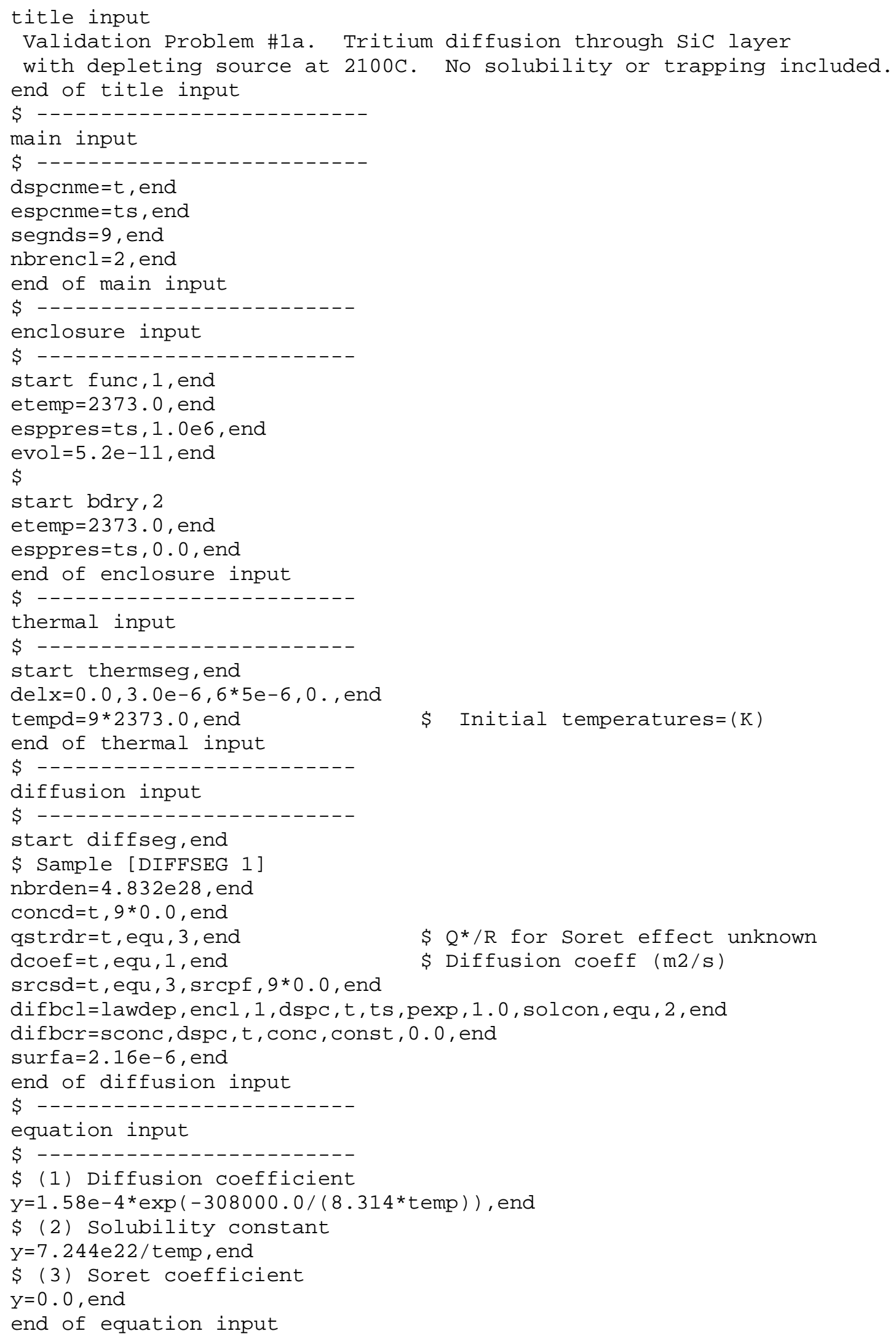




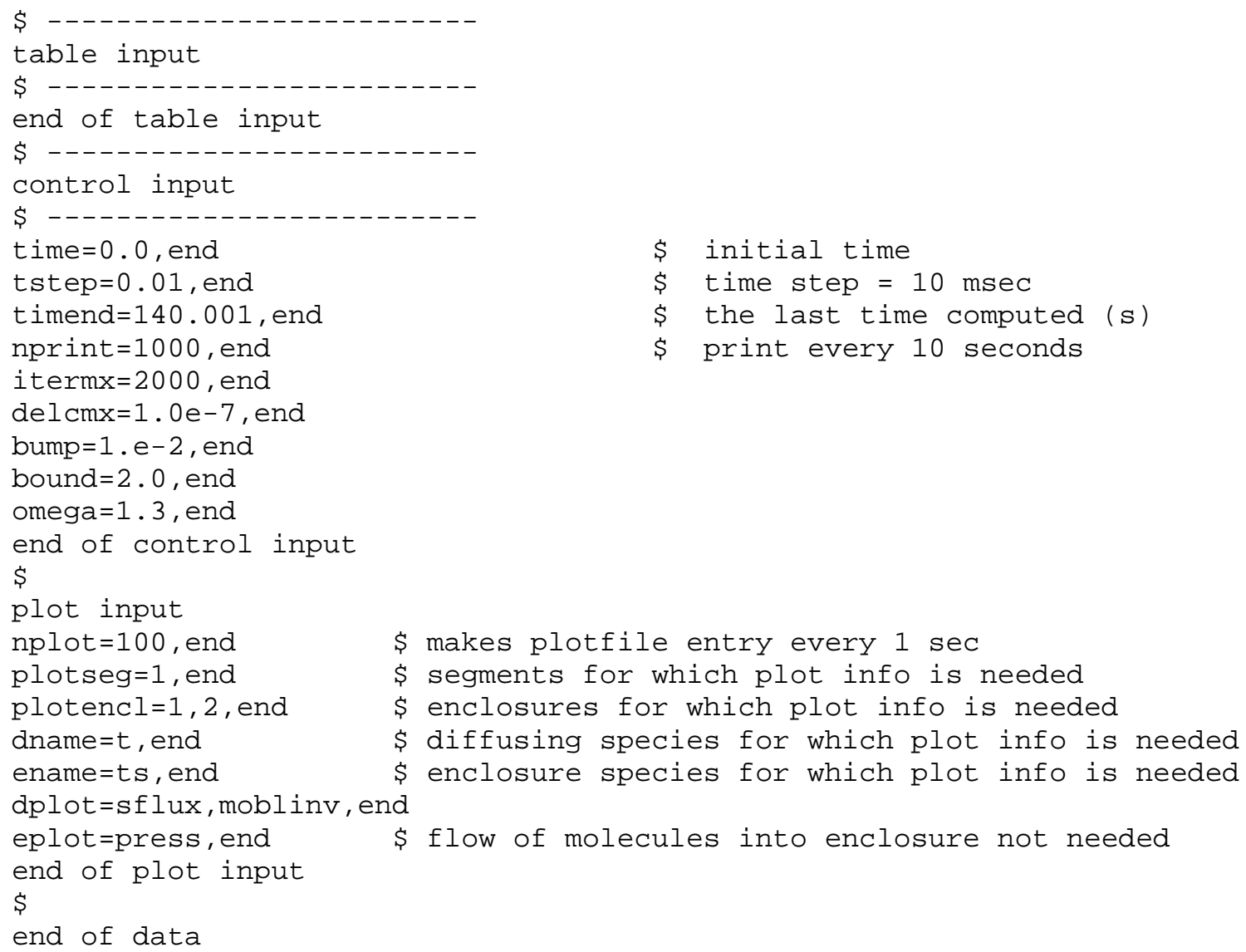


Problem 1b: Diffusion in a Semi-Infinite Slab with Constant-Source Boundary (Val-1b, see p. 5)

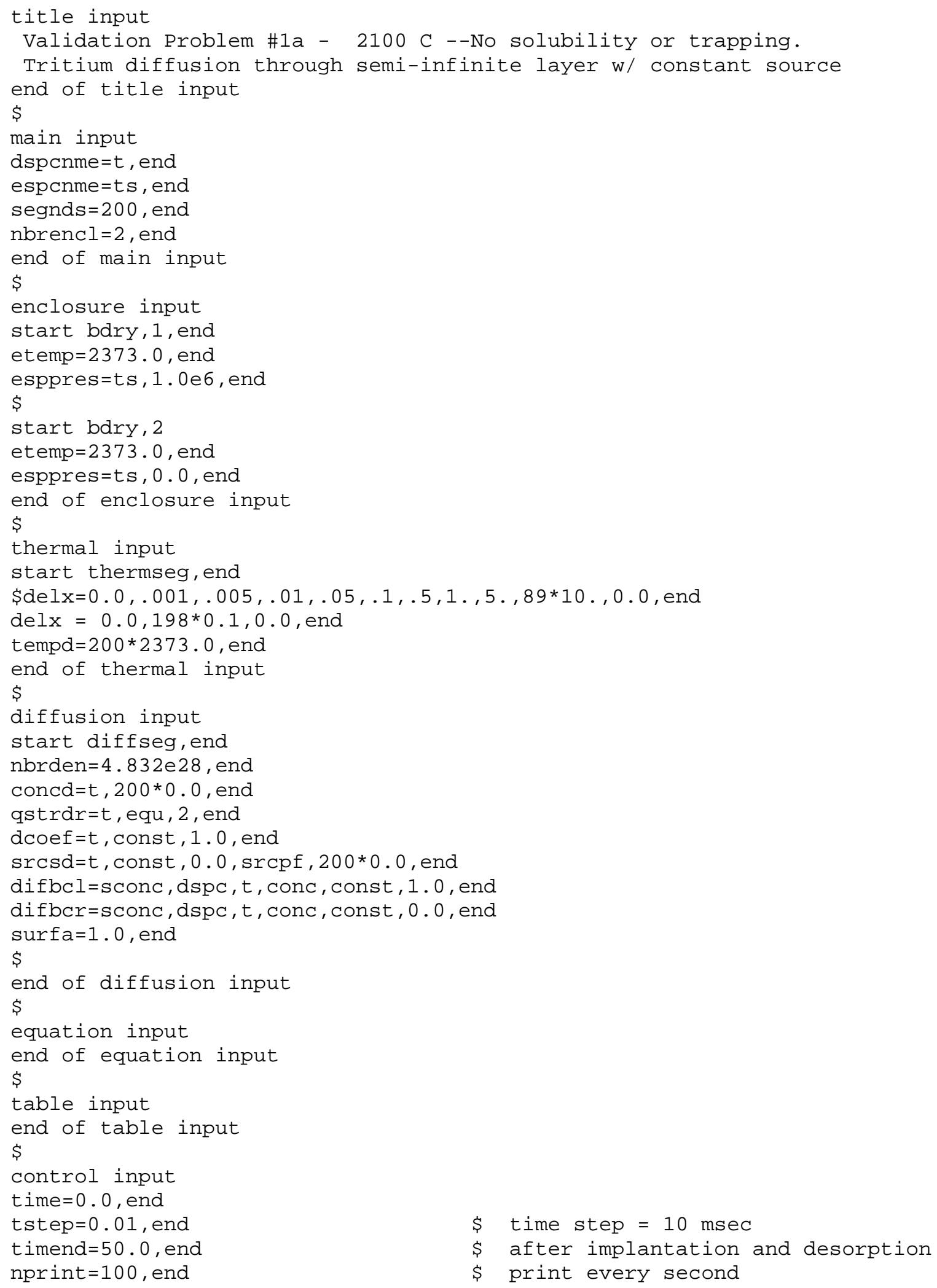




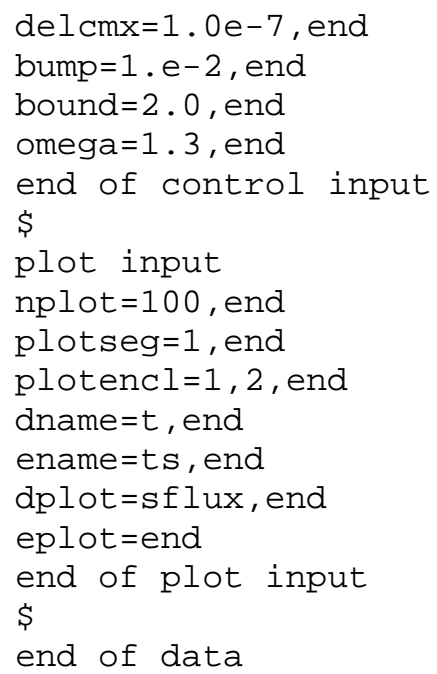

$\$$ makes plotfile entry every 1 sec

$\$$ segments for which plot info is needed

$\$$ enclosures for which plot info is needed

\$ diffusing species for which plot info is needed

$\$$ enclosure species for which plot info is needed

\$ flow of molecules into enclosure not needed 
Problem 1c: Diffusion in a Partially Preloaded Semi-Infinite Slab (Val-1c, see p. 8)

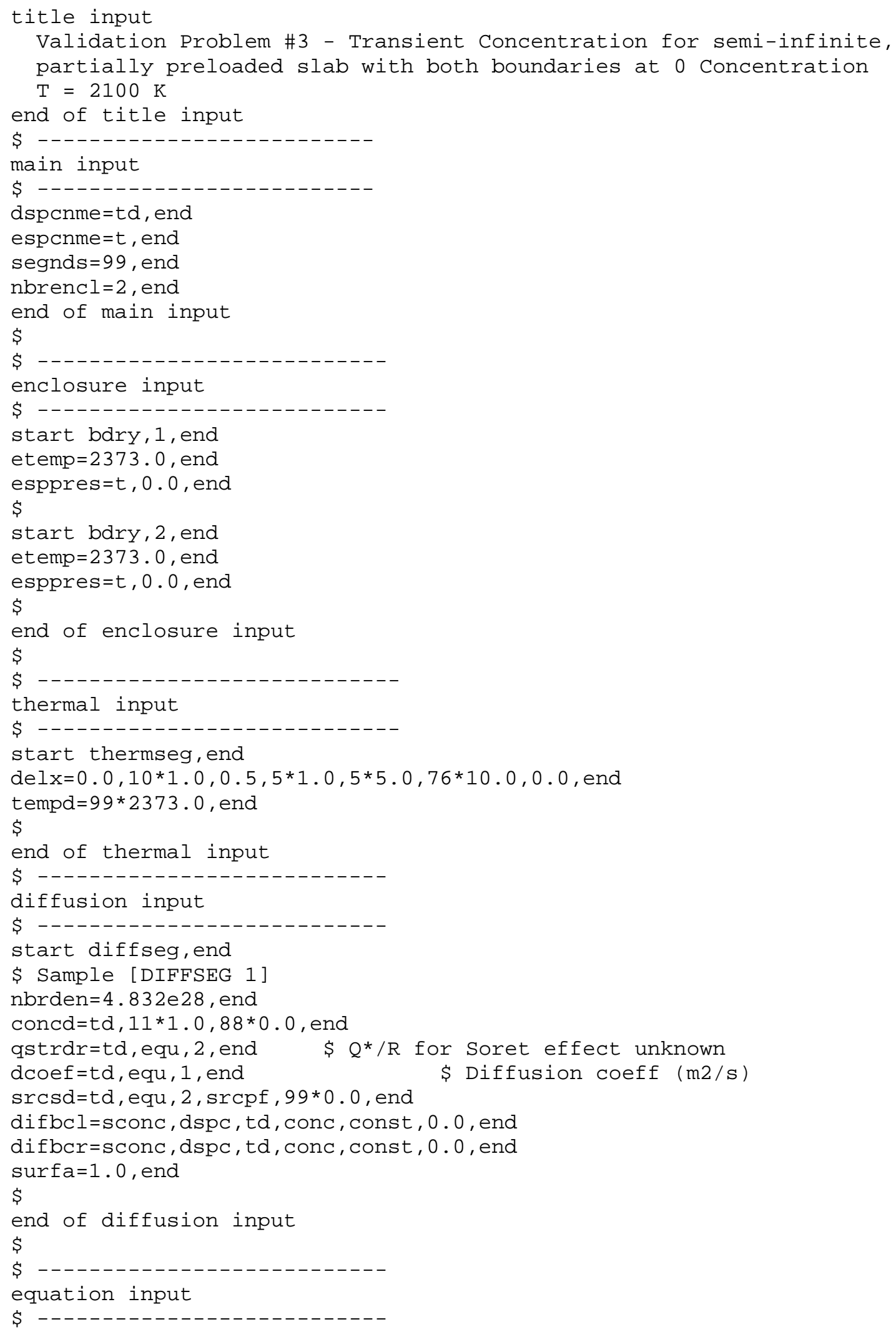




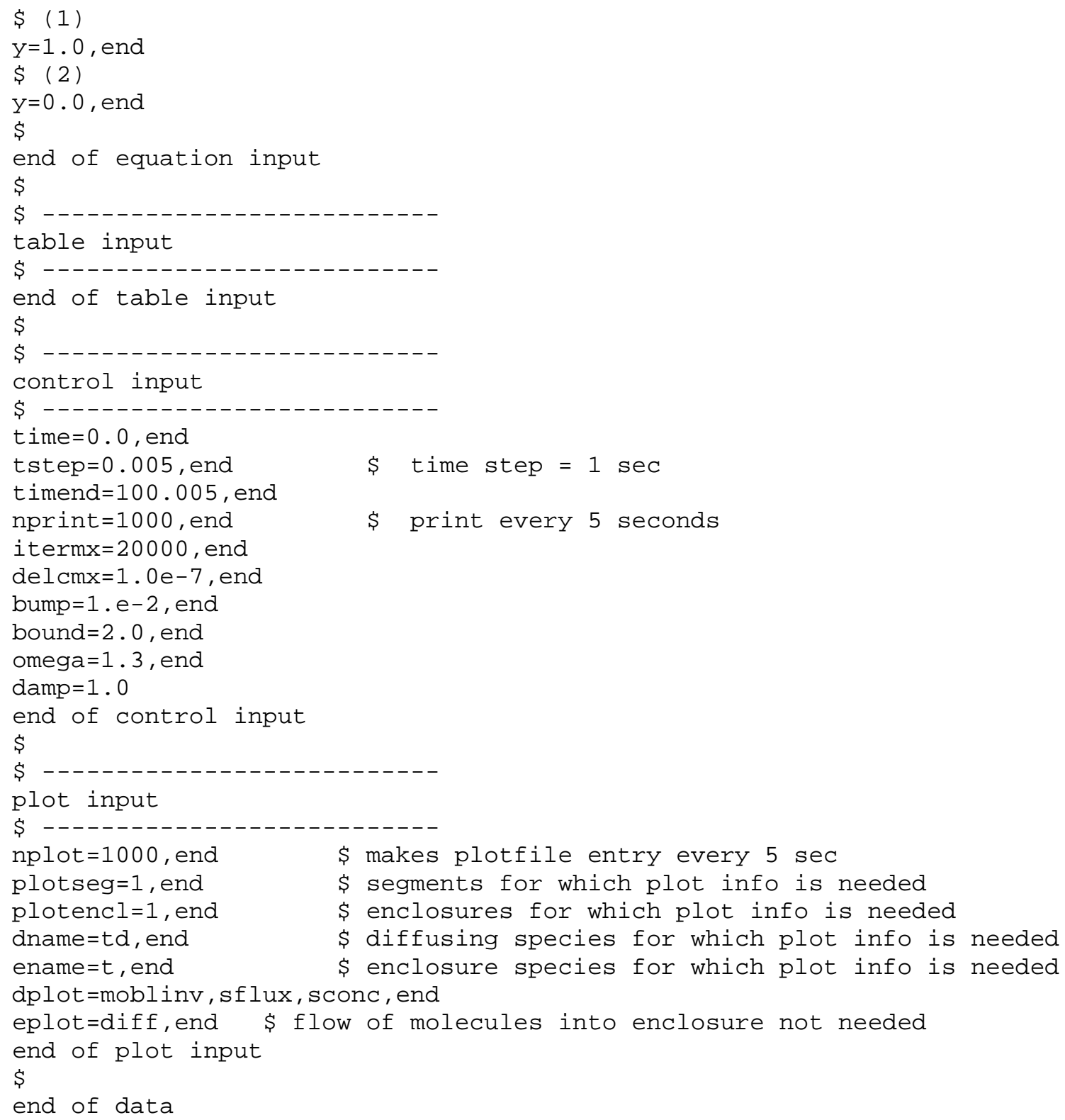


Problem 1da. Effective Diffusivity Trap (Val-1da, see p. 12)

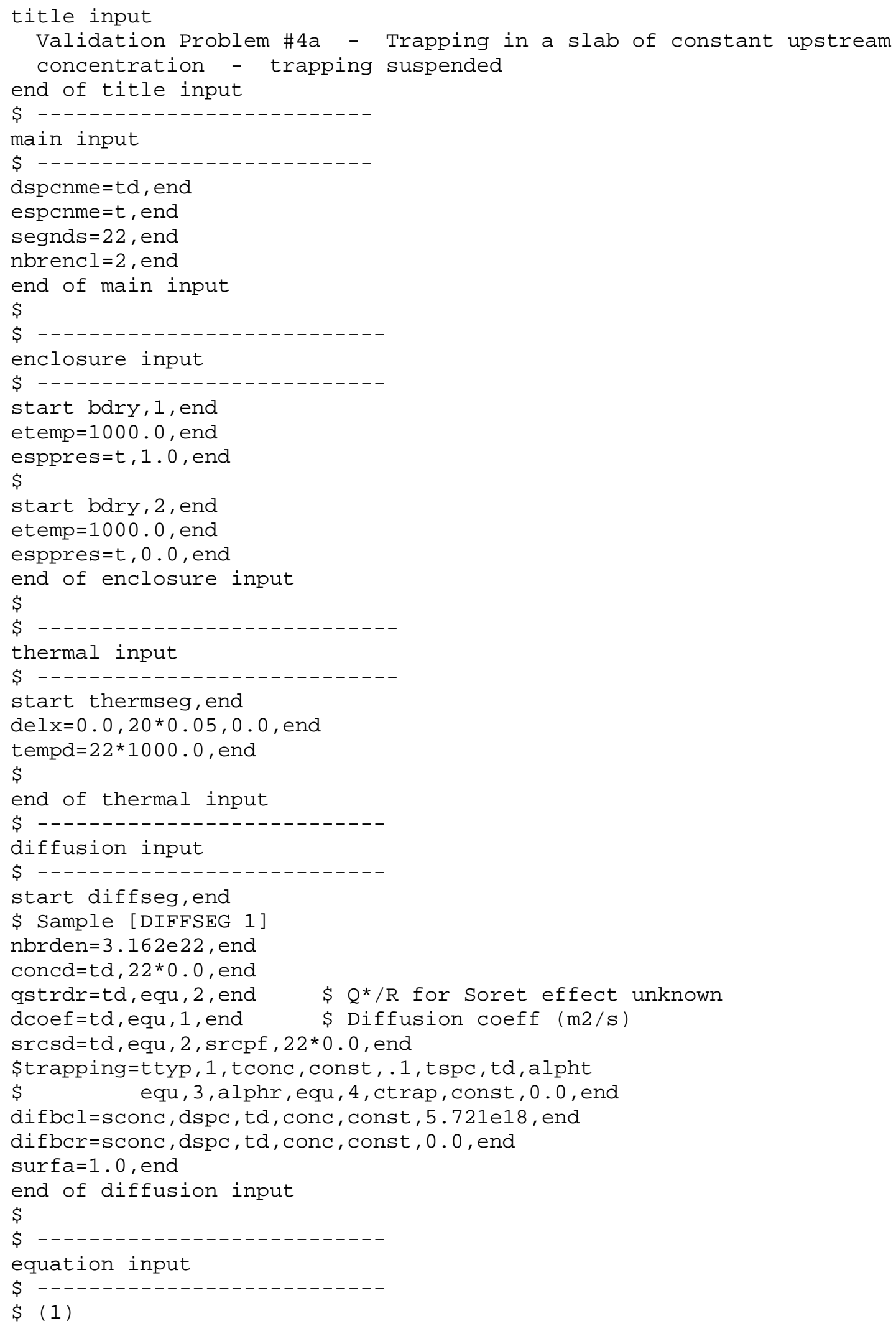




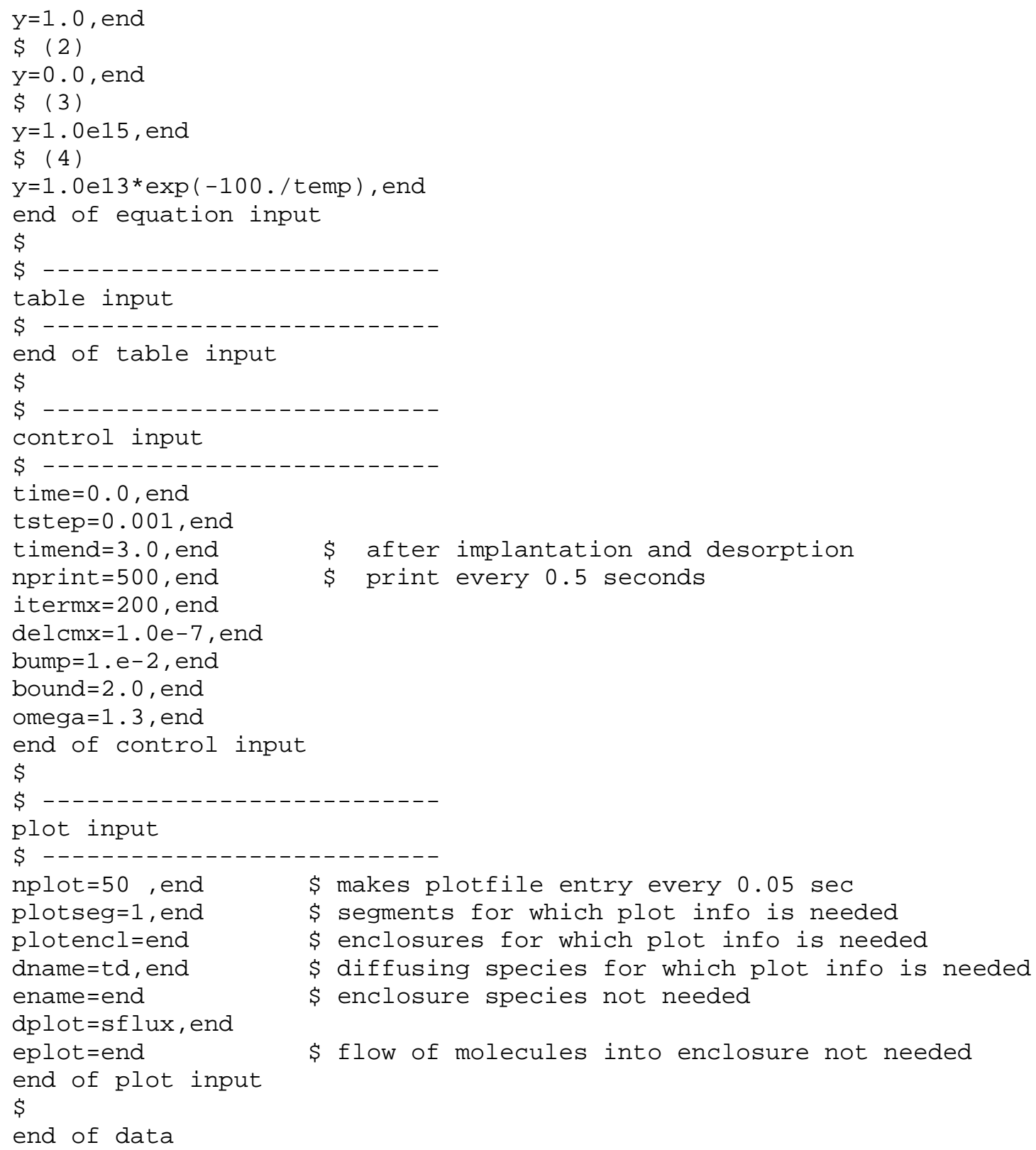


Problem 1db. Strong Trap (Val-1db, see p. 13)

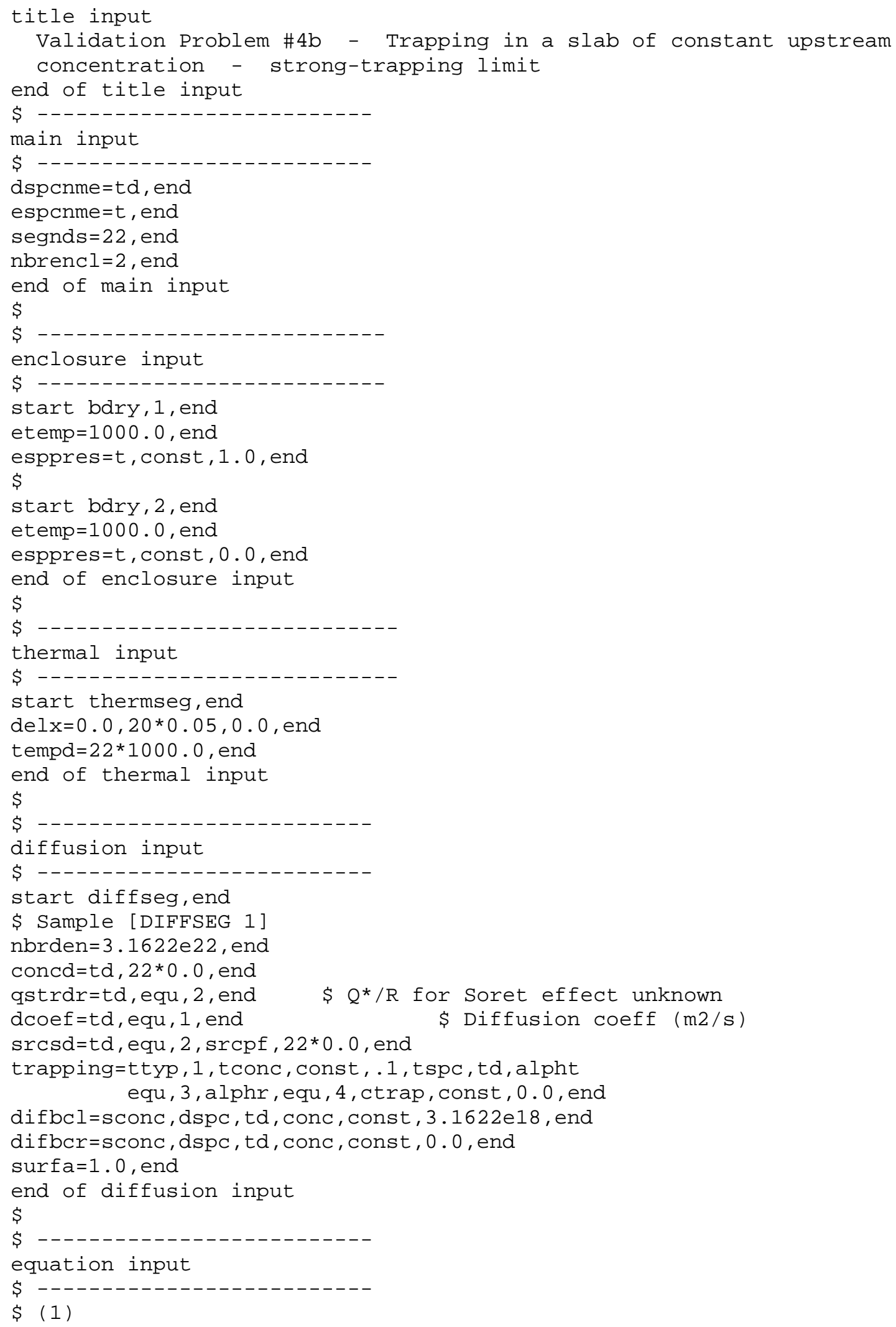




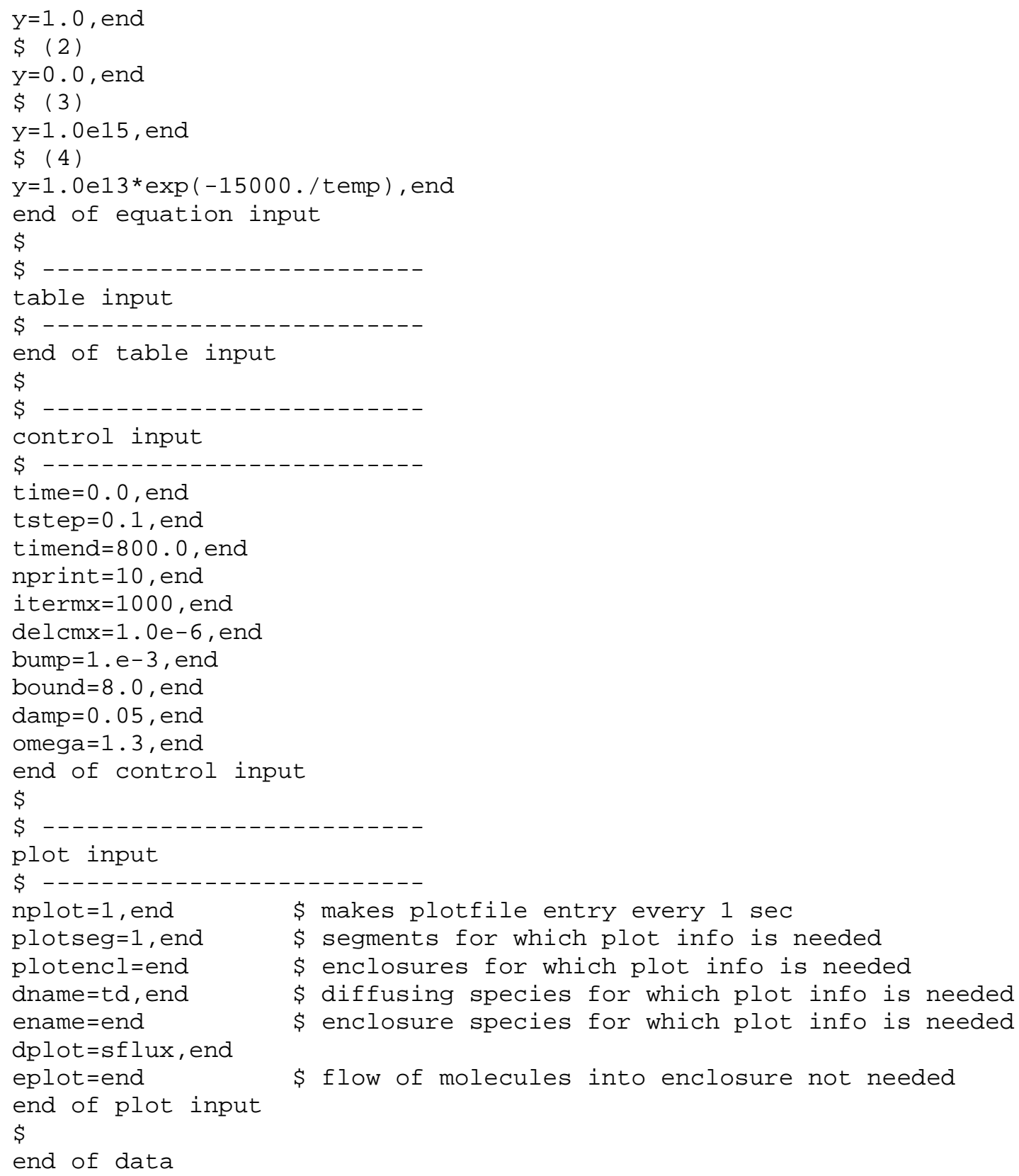


Problem 1dc. Multiple Trap (Val-1dc, see p. 14)

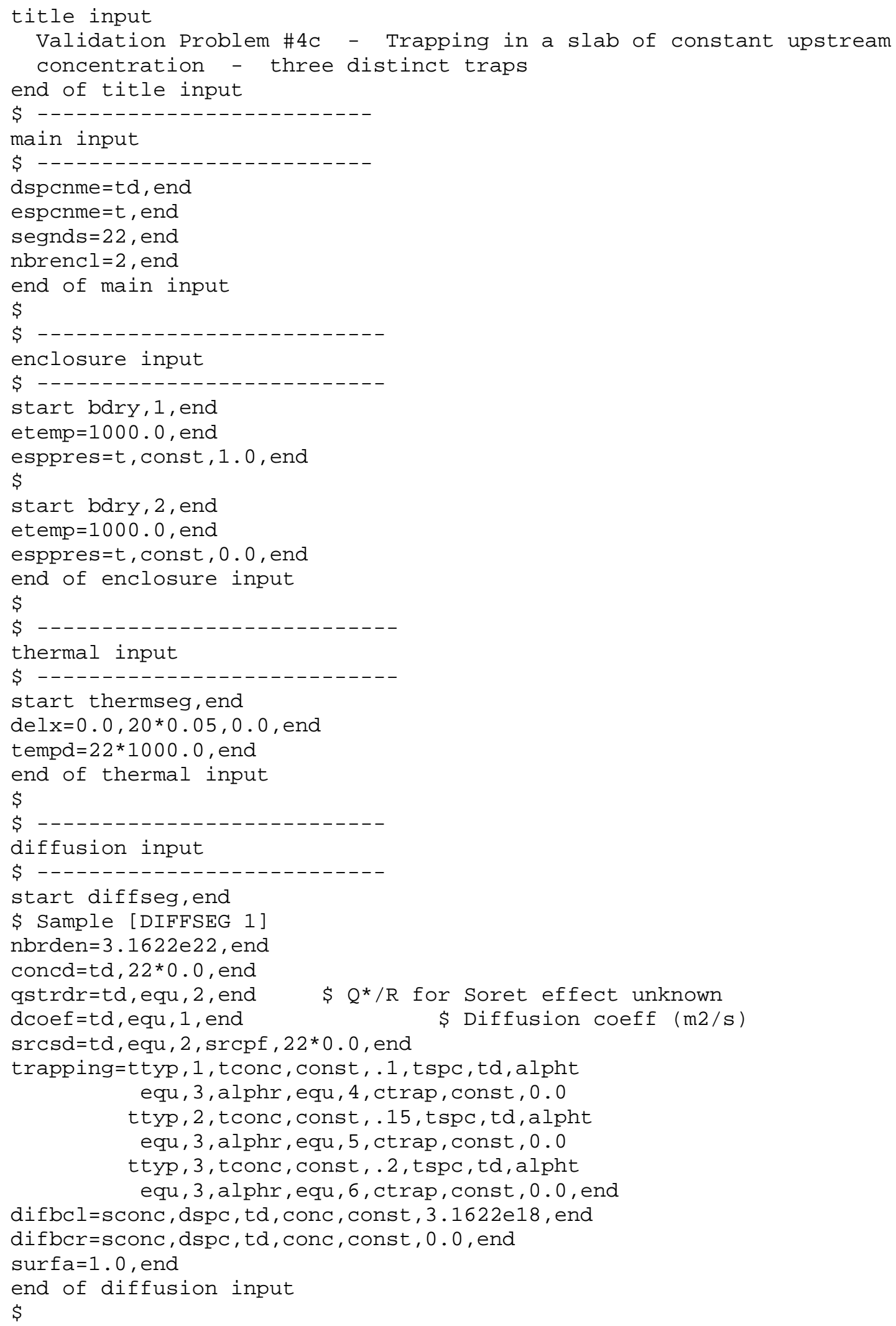




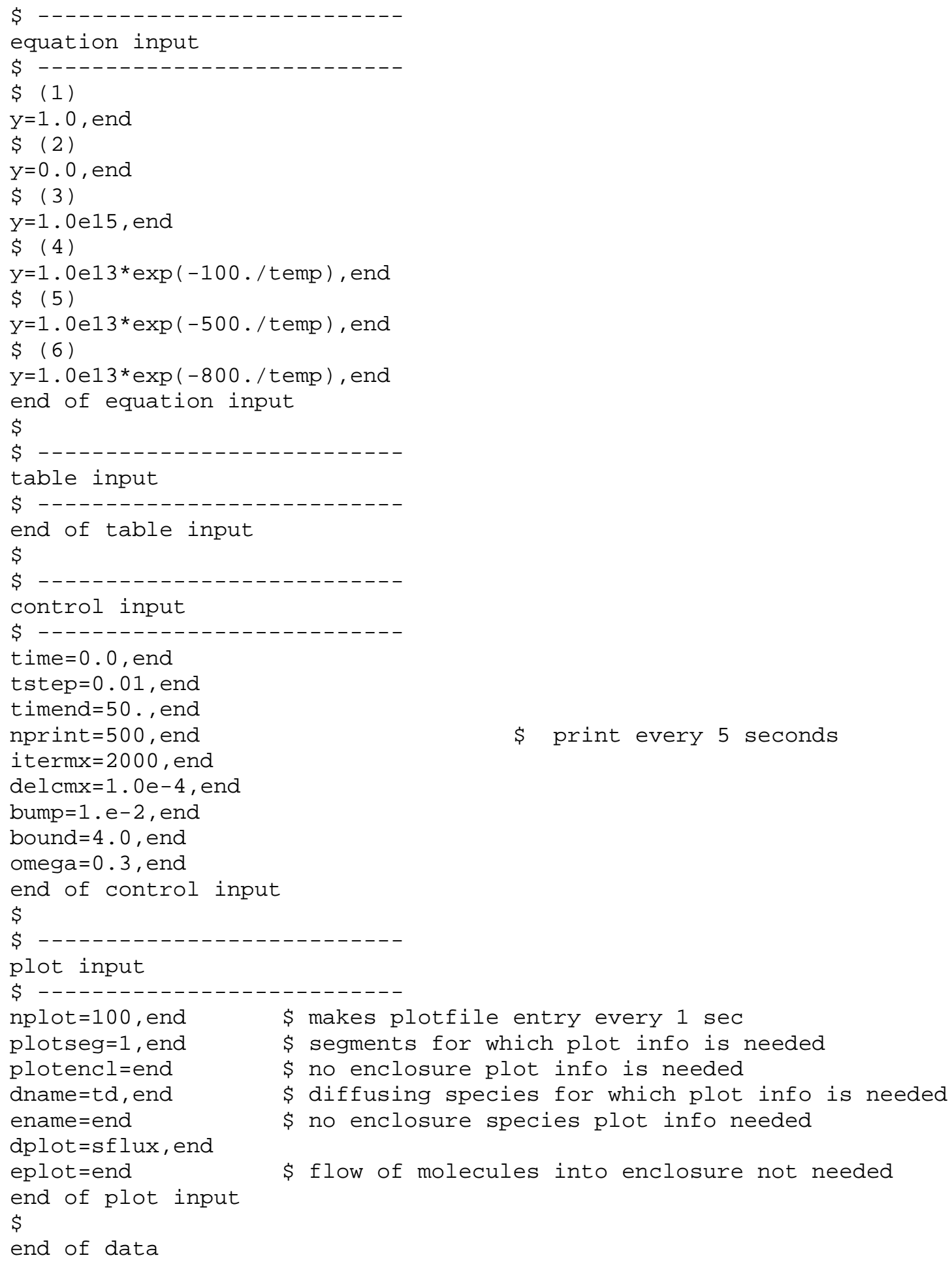


Problem 1e: Diffusion with Composite Material Layers (Val-1e, see p. 15)

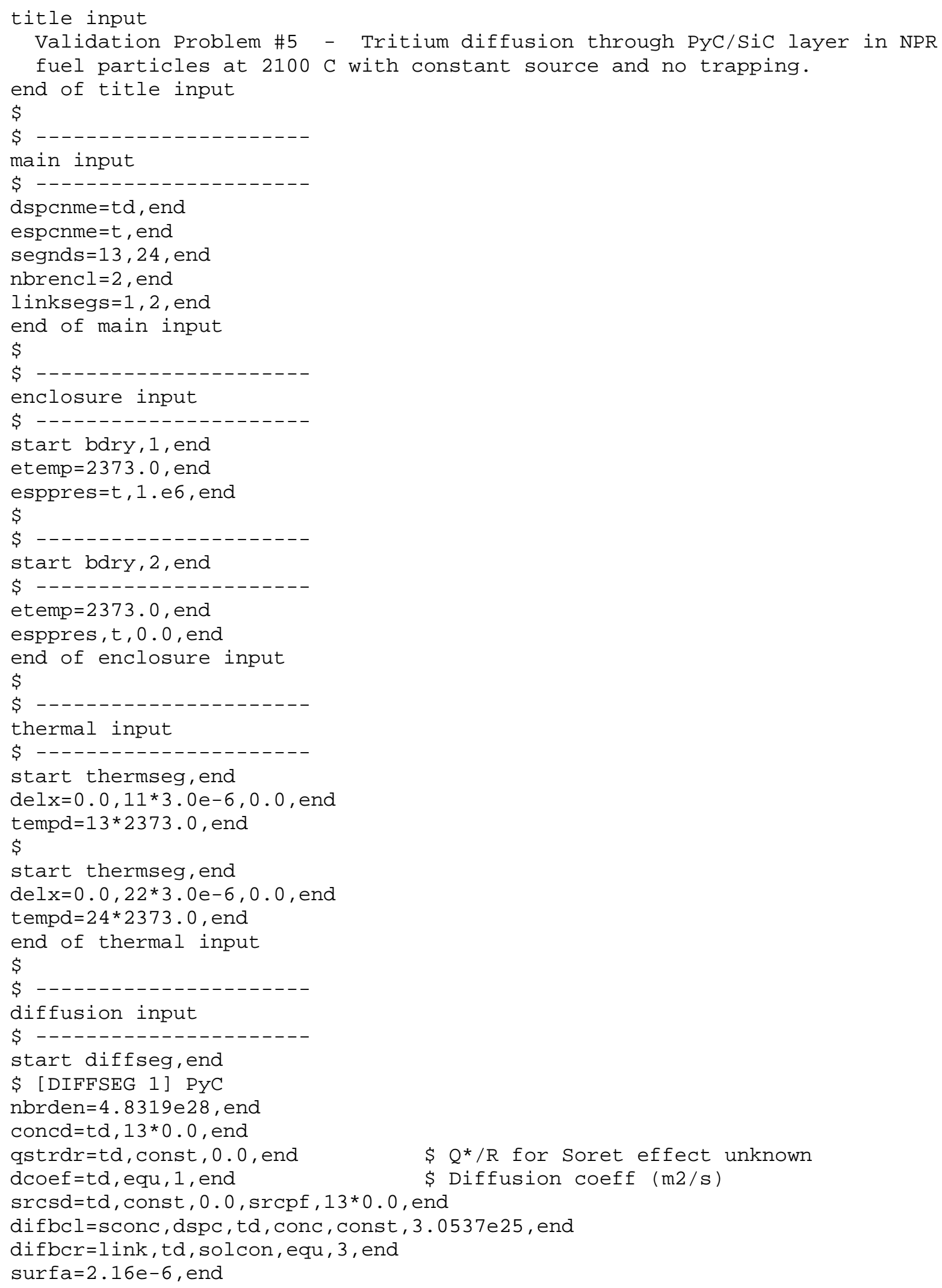




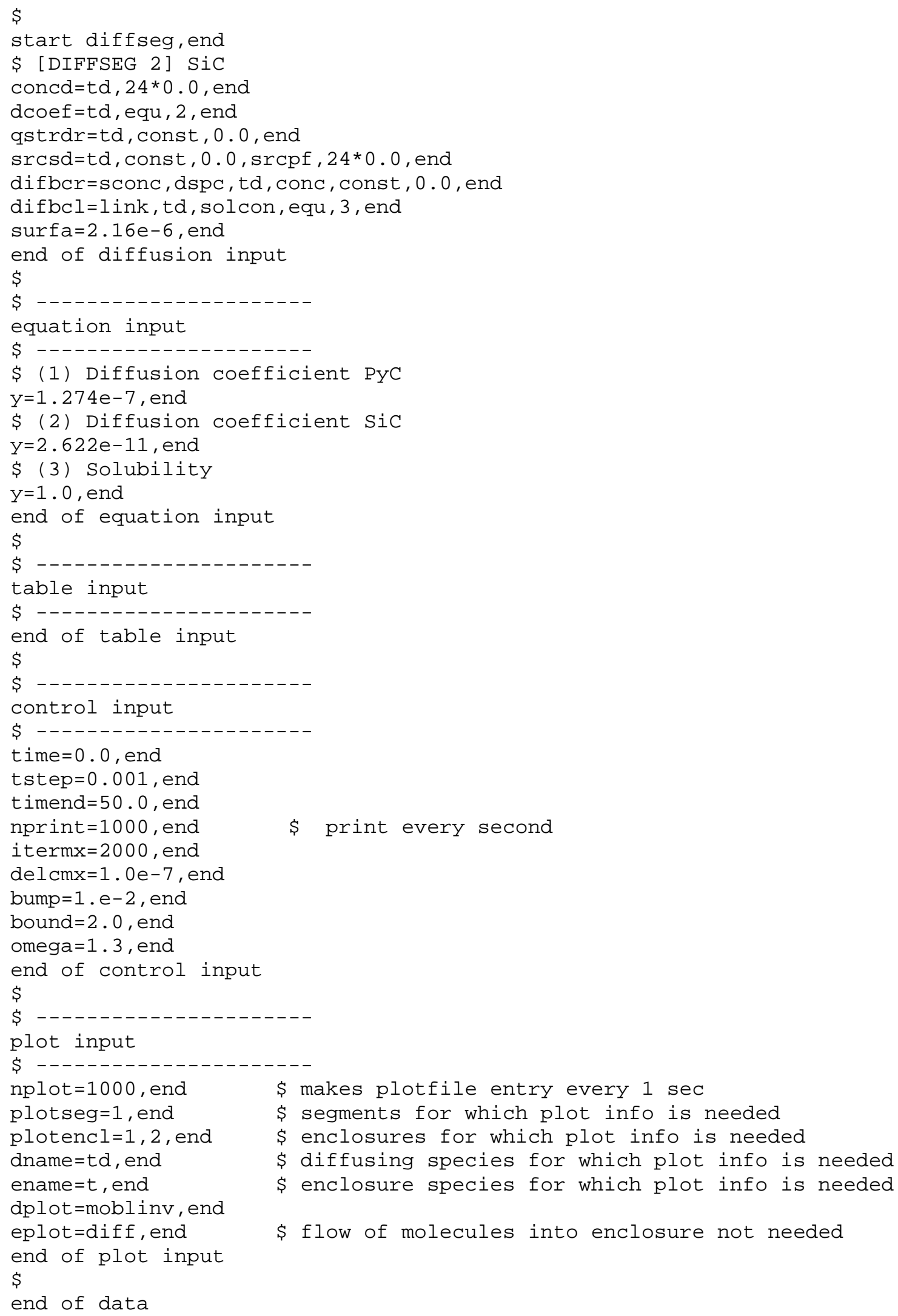


Problem 1f: Heat Sink/Source Problem (Val-1fa, see p. 18)

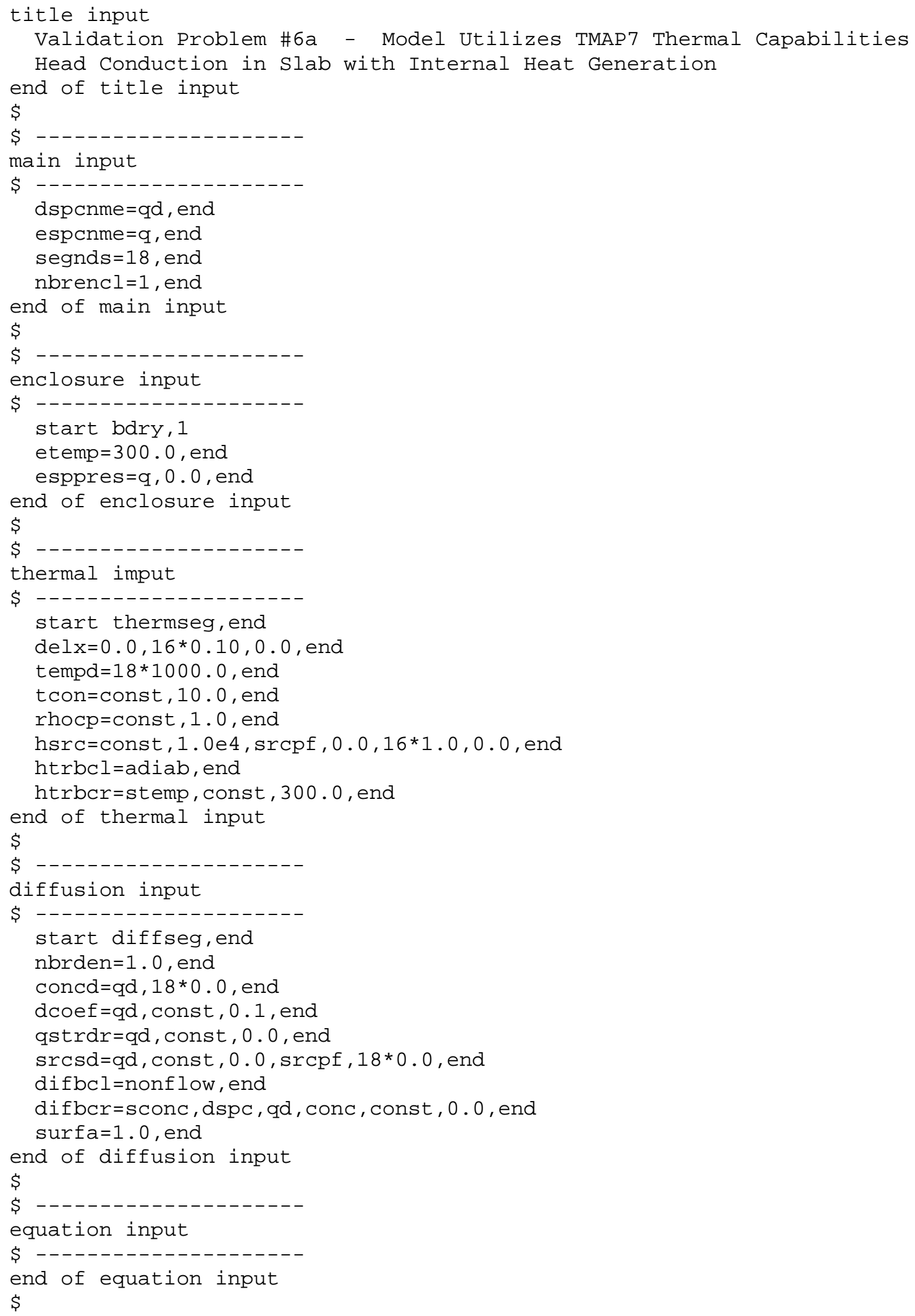




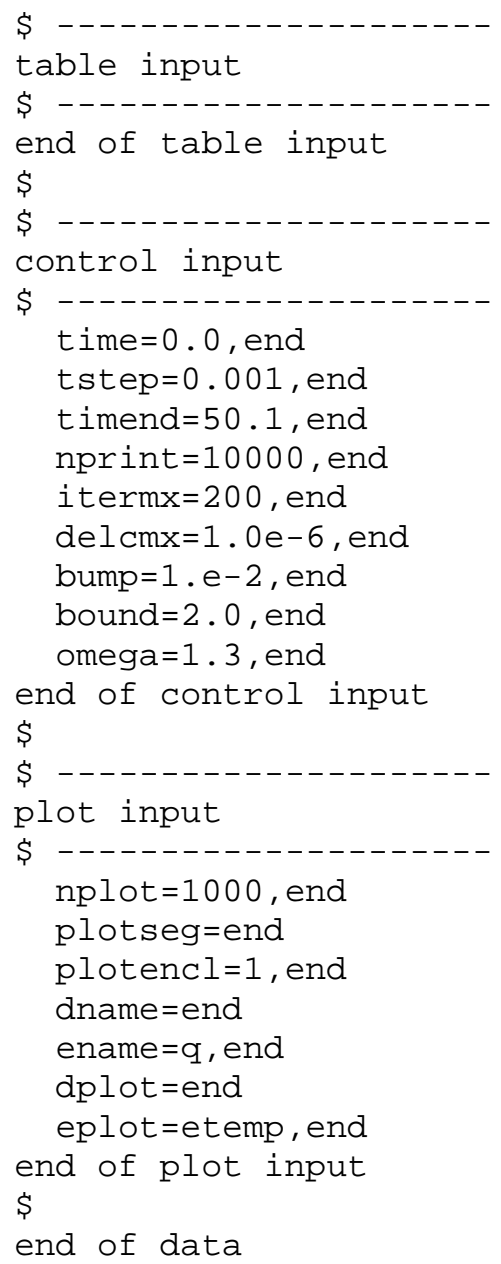


Problem $1 \mathrm{fb}$. Thermal Diffusion Transient (Val-1fb, see p. 19)

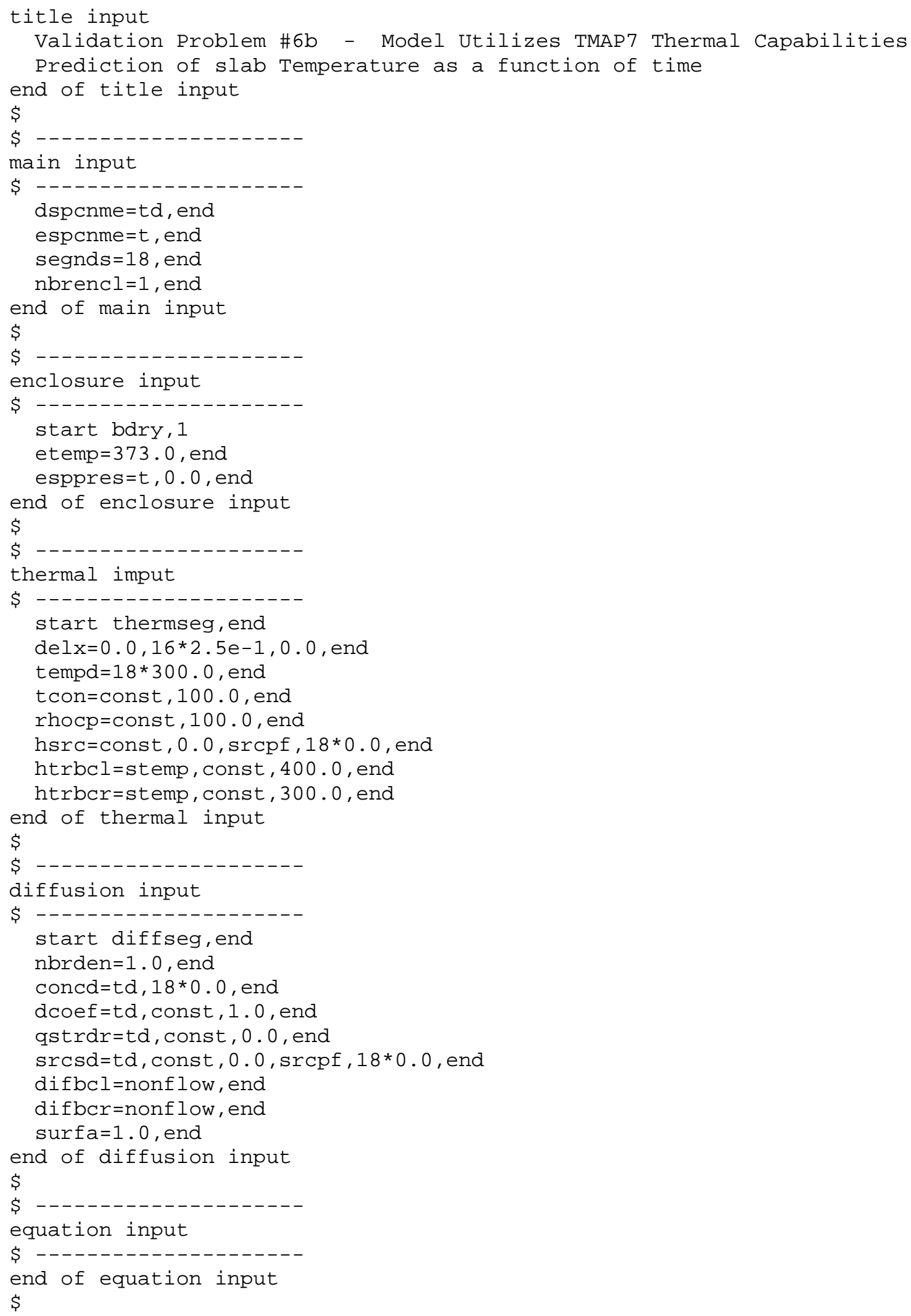




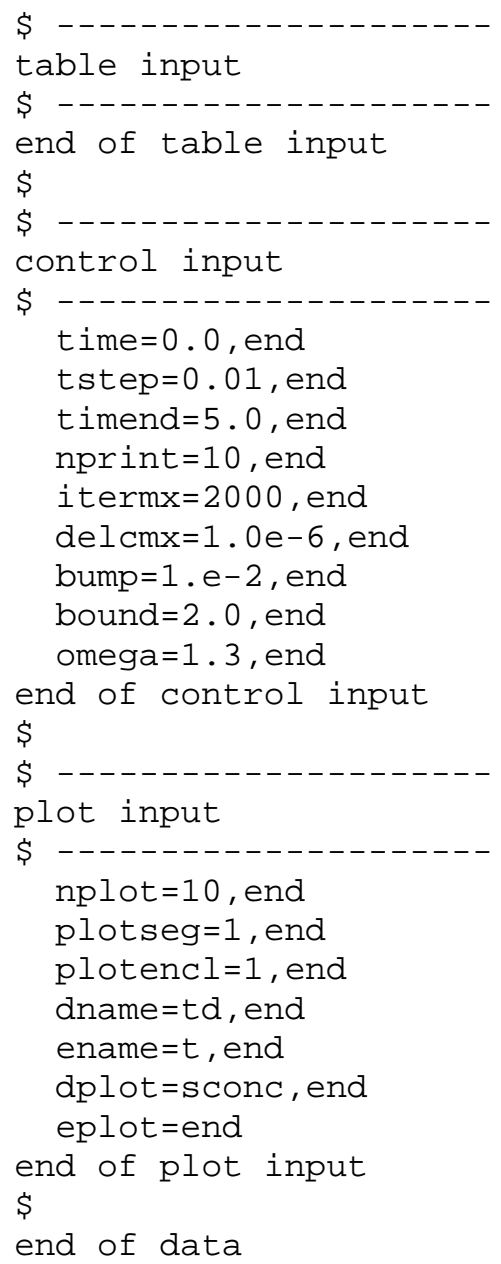


Conduction in Composite Structure with Constant Surface Temperatures (Val-1fc, see p. 20)

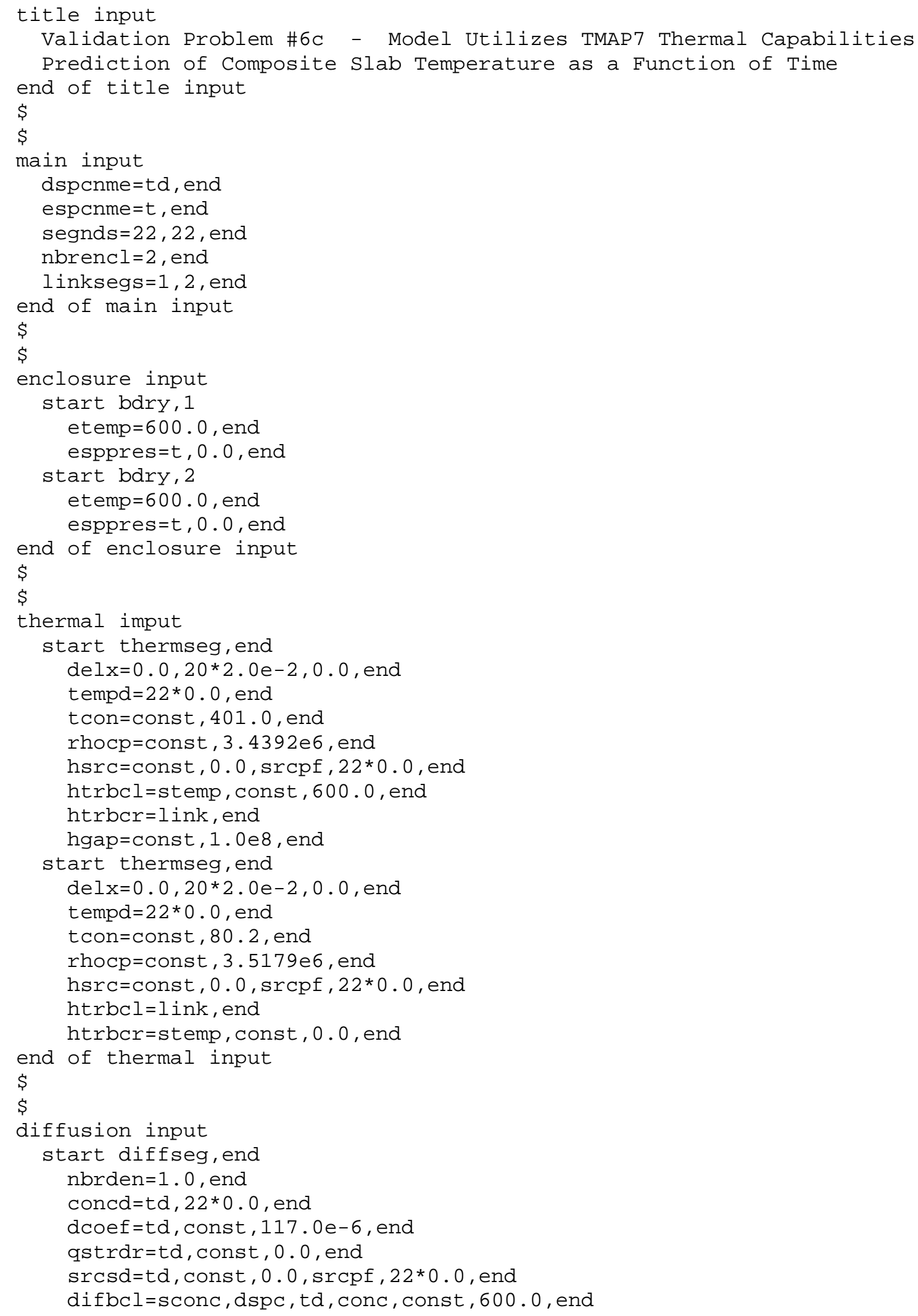




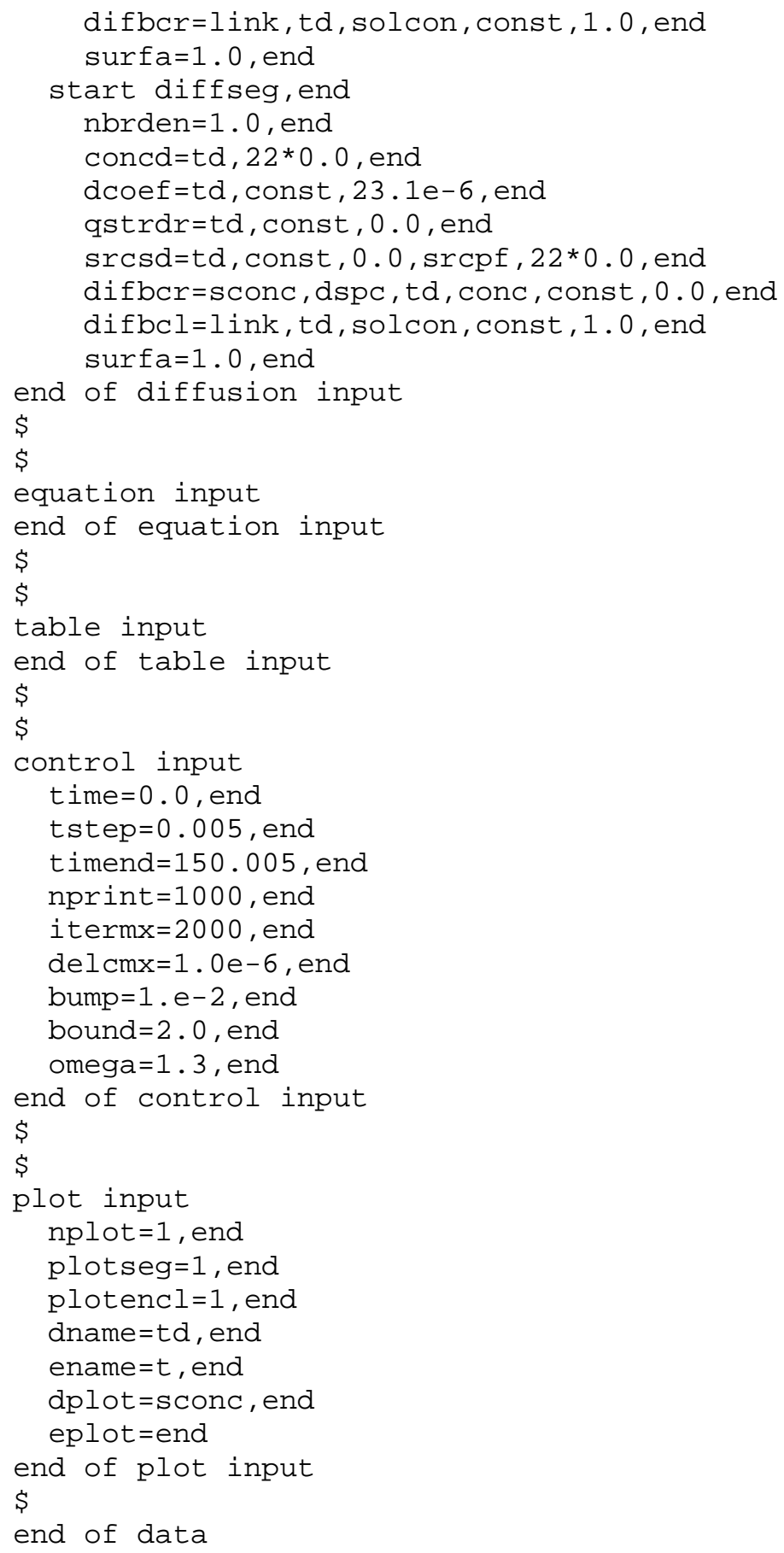


Heat Conduction in Semi-Infinite Copper Slab with Convectionnvection (Val-1fd, see p. 24)

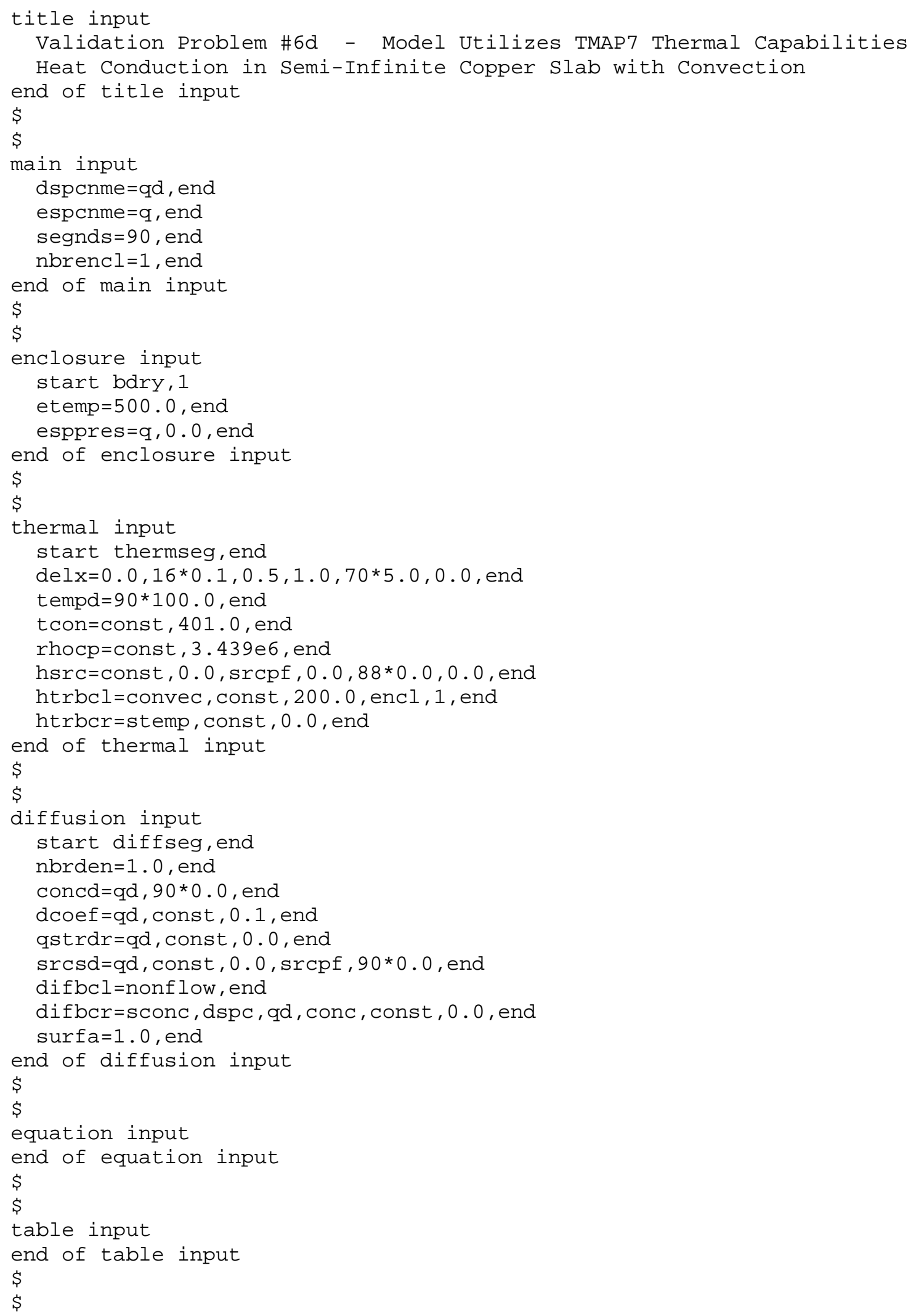




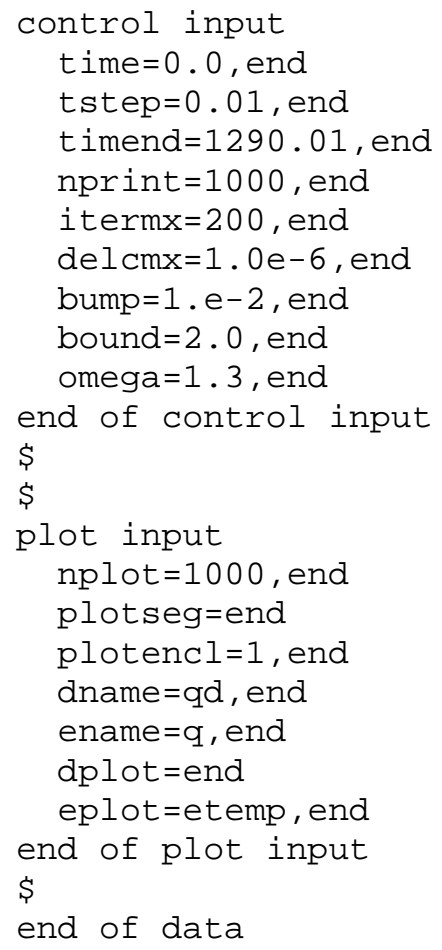


Problem 1ga. Simple Forward Reactions (Val-1ga, see p.26)

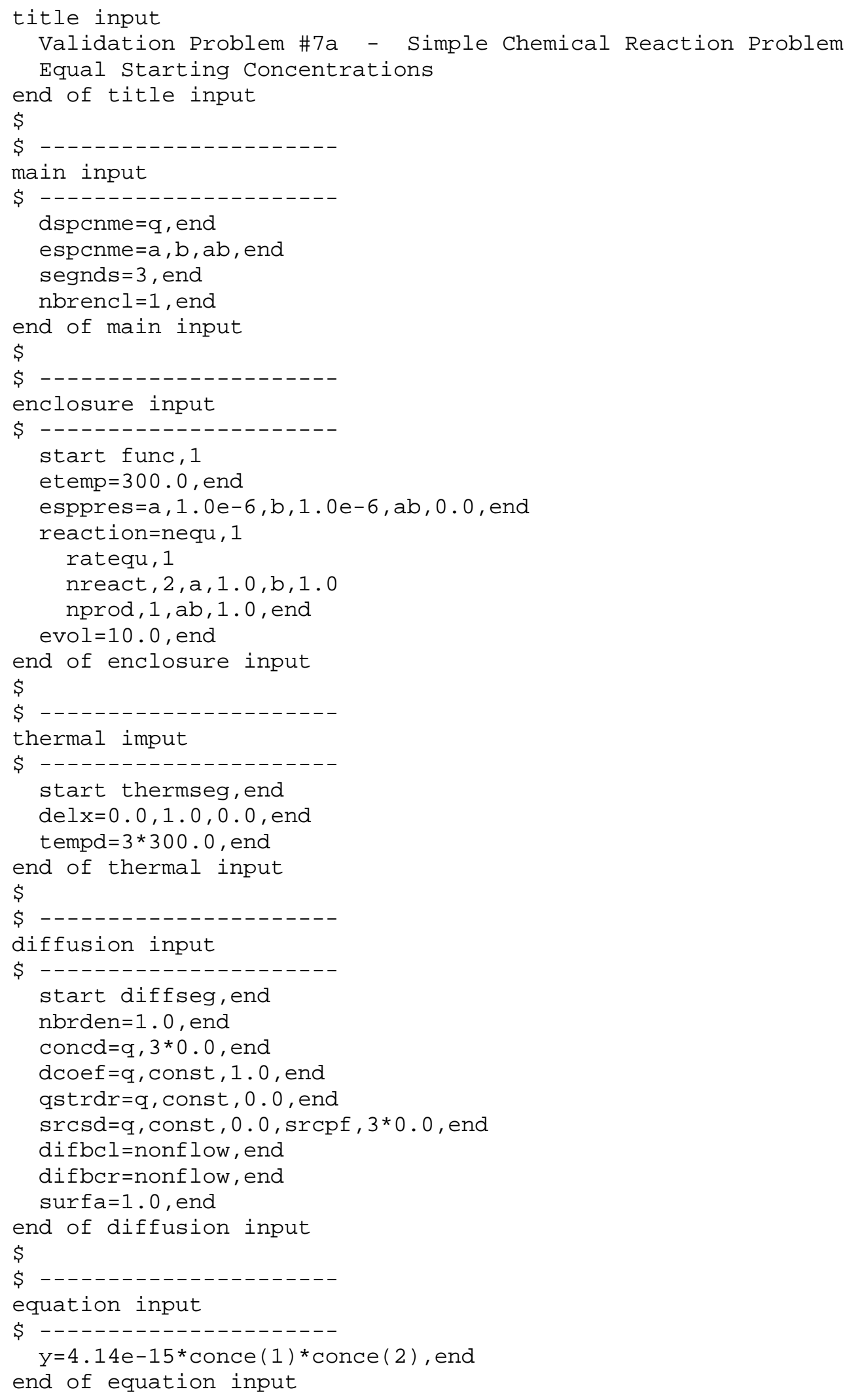




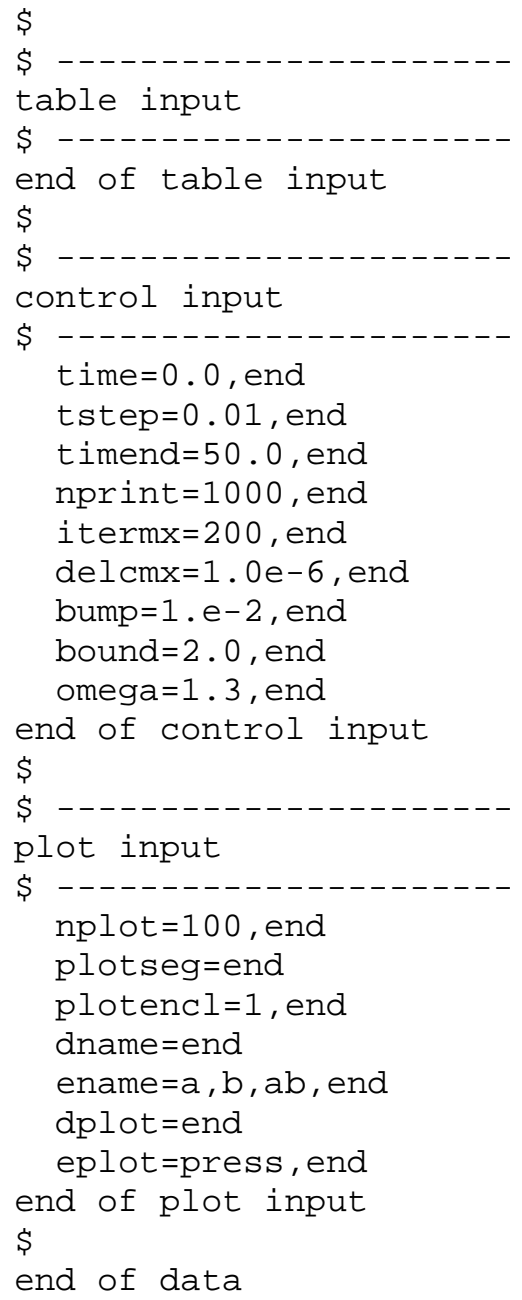


Problem 1gb. Simple Forward Reactions (Val-1gb, see p. 26)

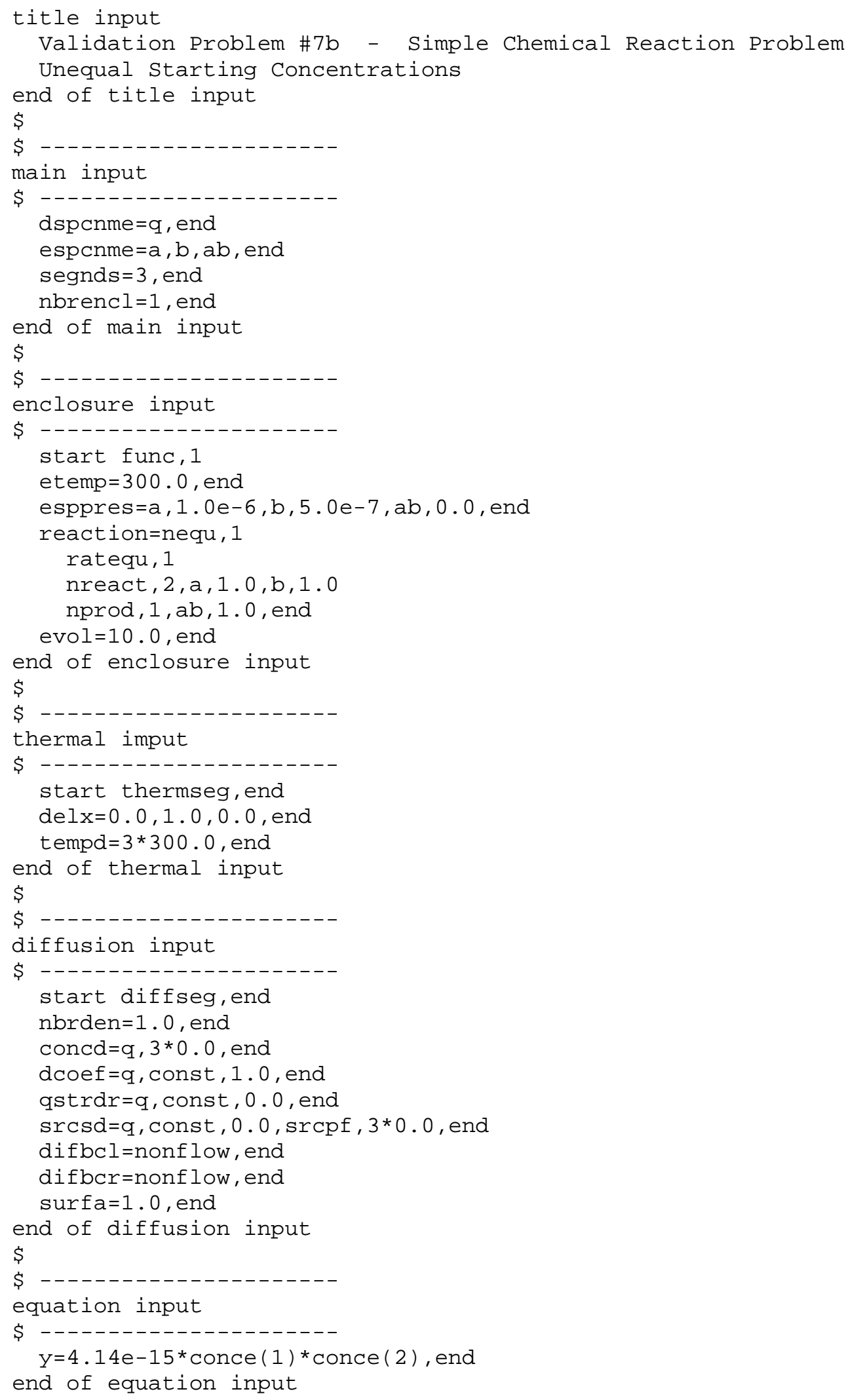




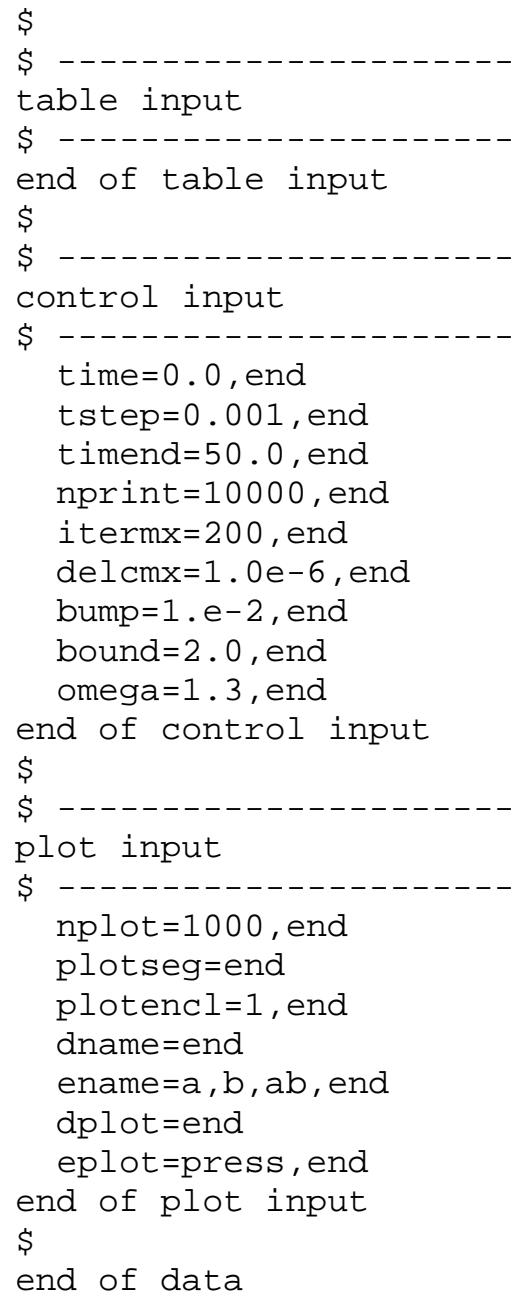


Problem 1gc. Series Reactions (Val-gc, see p. 29)

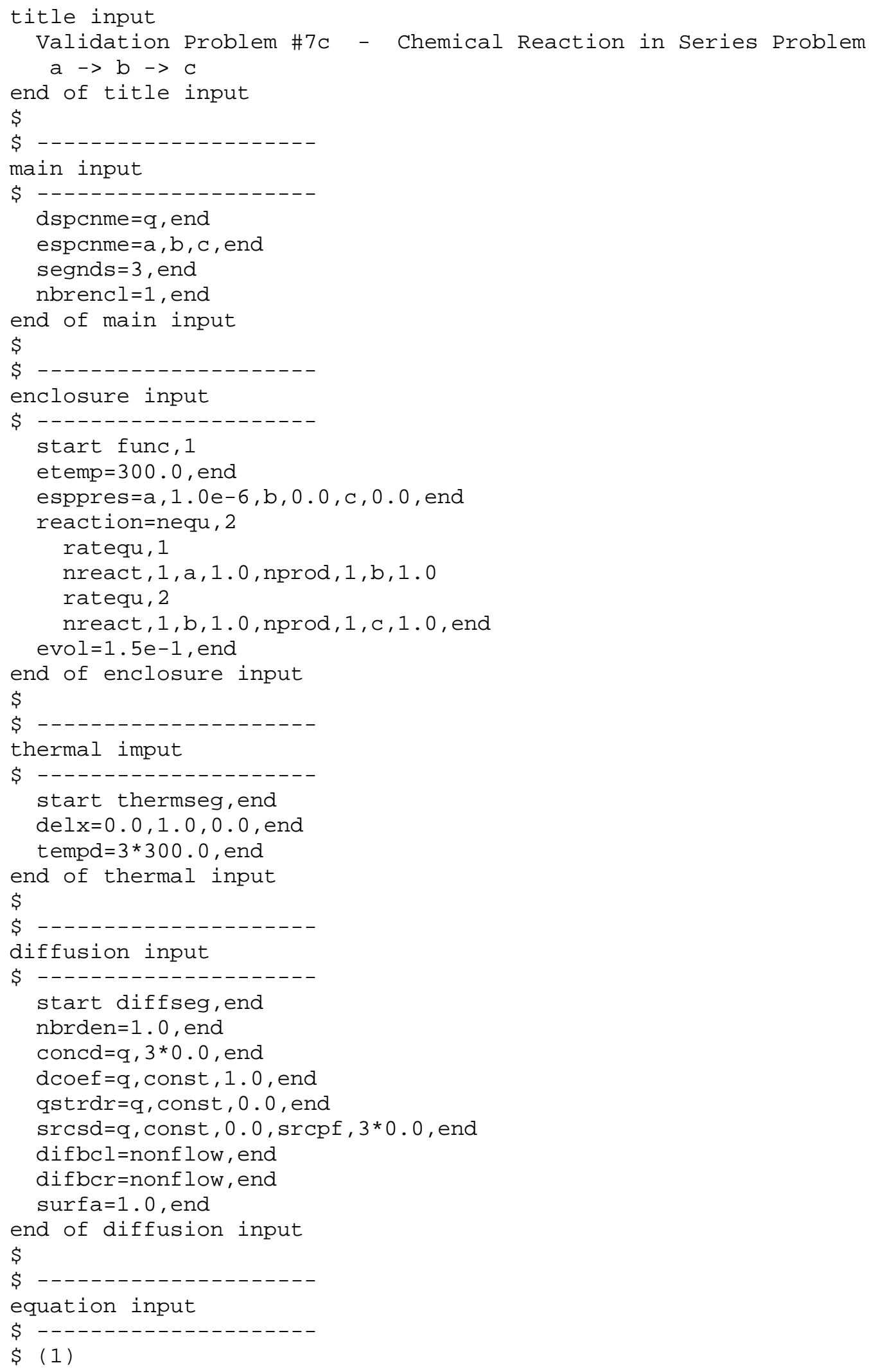




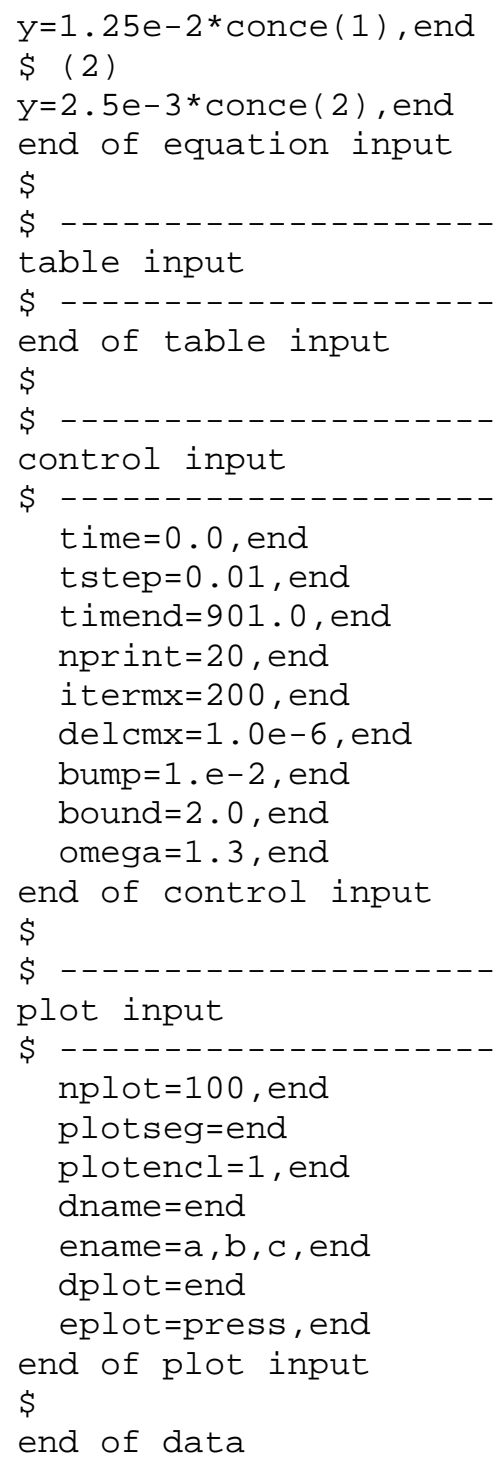


Problem 1ha. Three Enclosure Problem (Val-1ha, See p. 32)

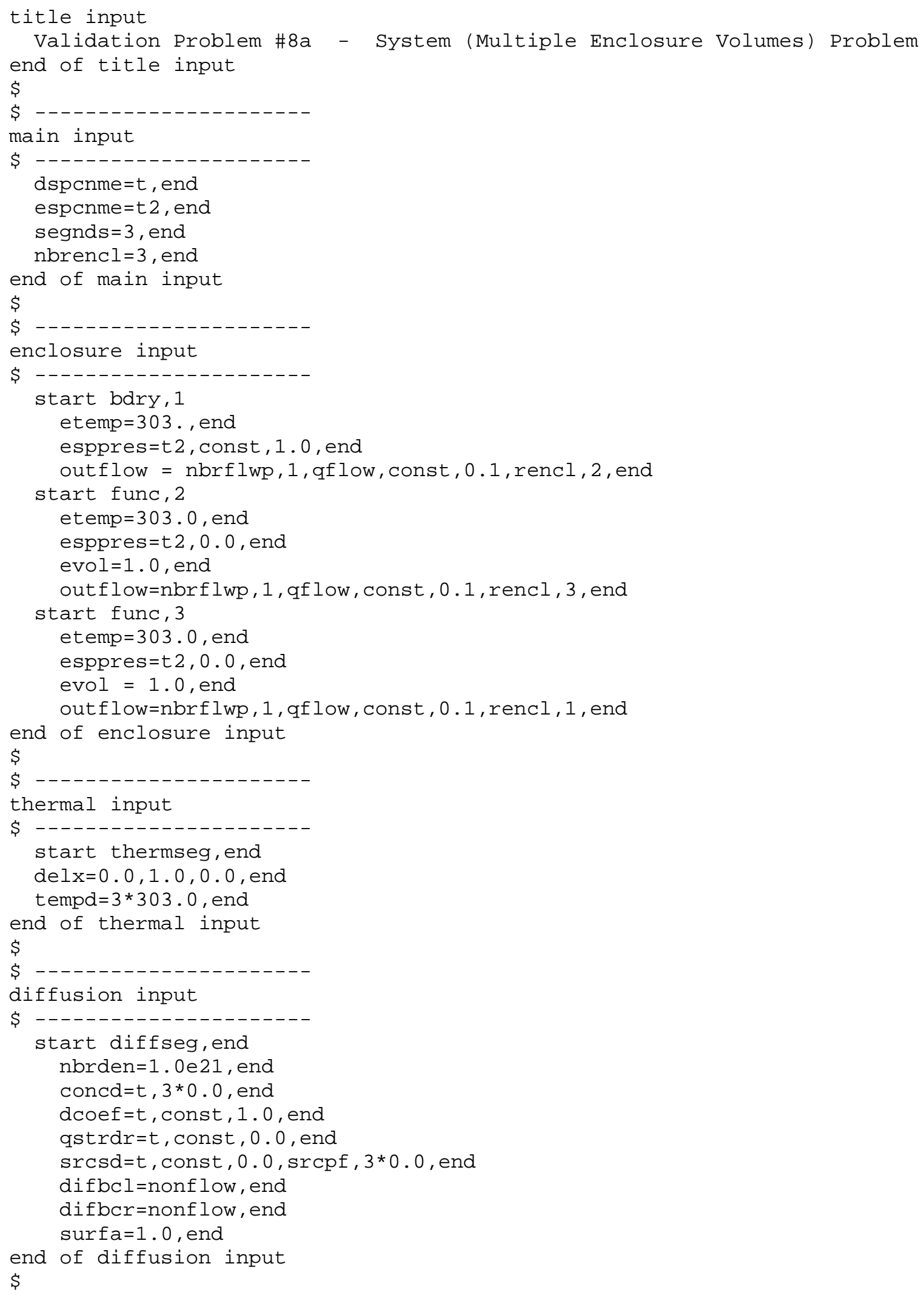




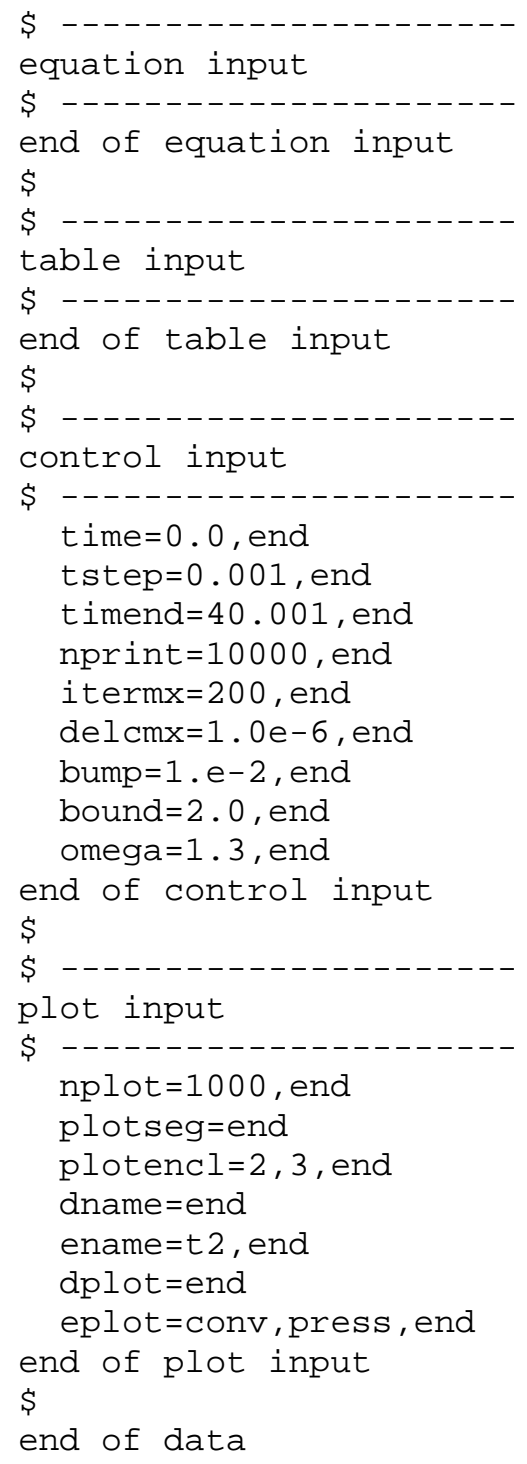


Problem 1hb. Equilibrating Enclosures (Val-1hb, see p. 33)

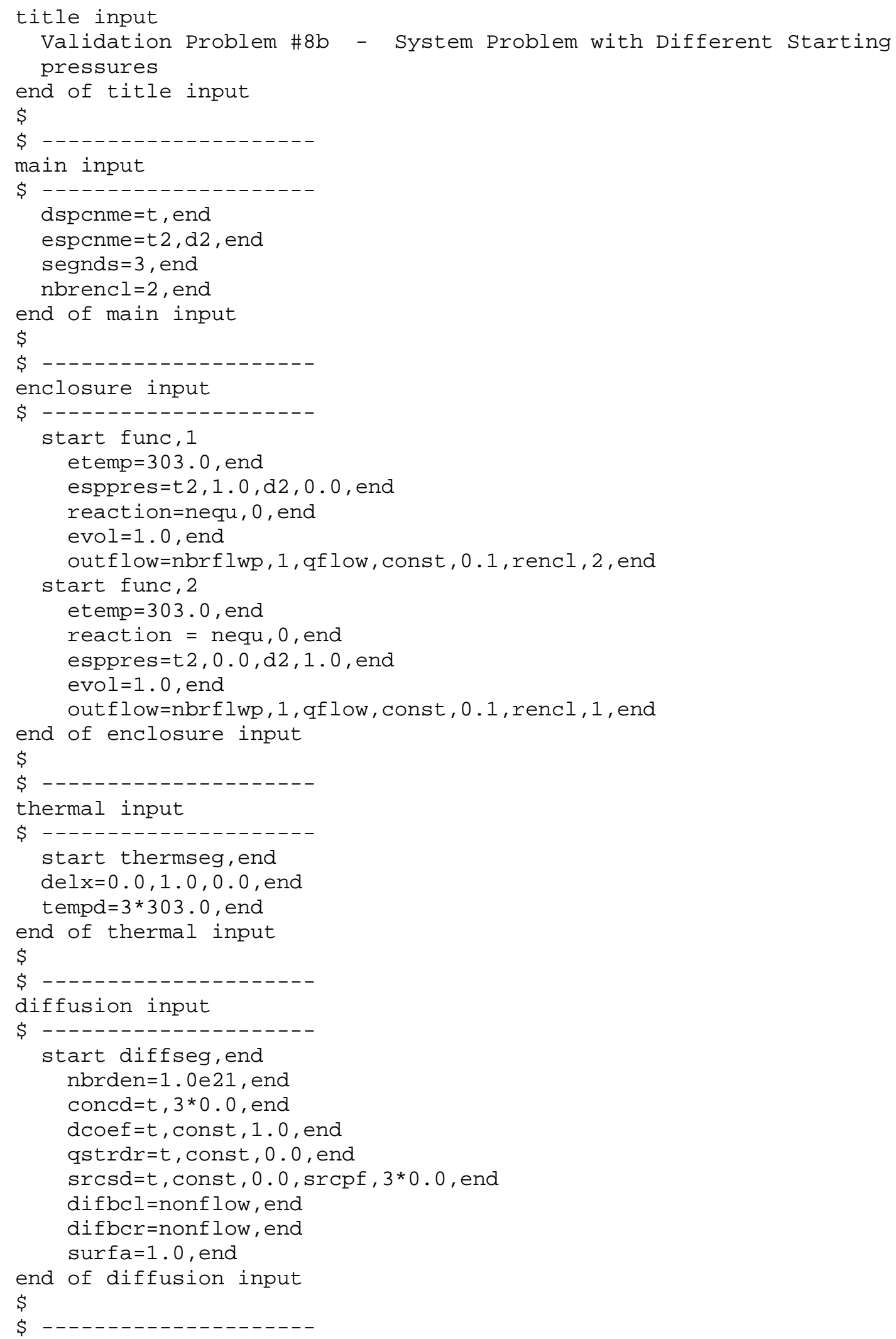




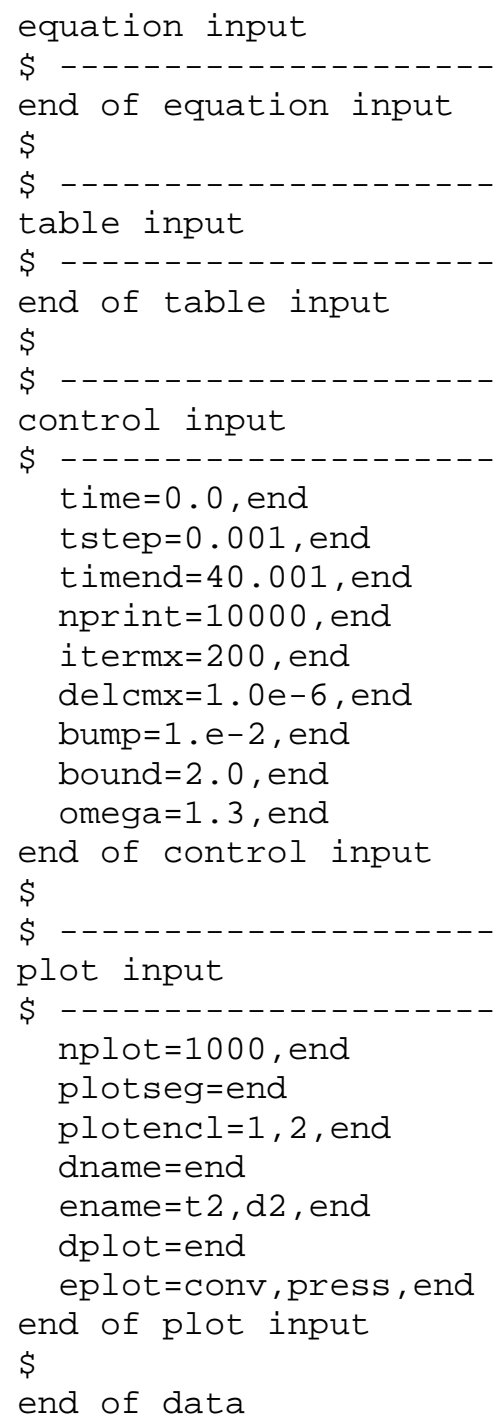


Species Equilibration on a Reactive Surface (Val-1ia, see p. 37)

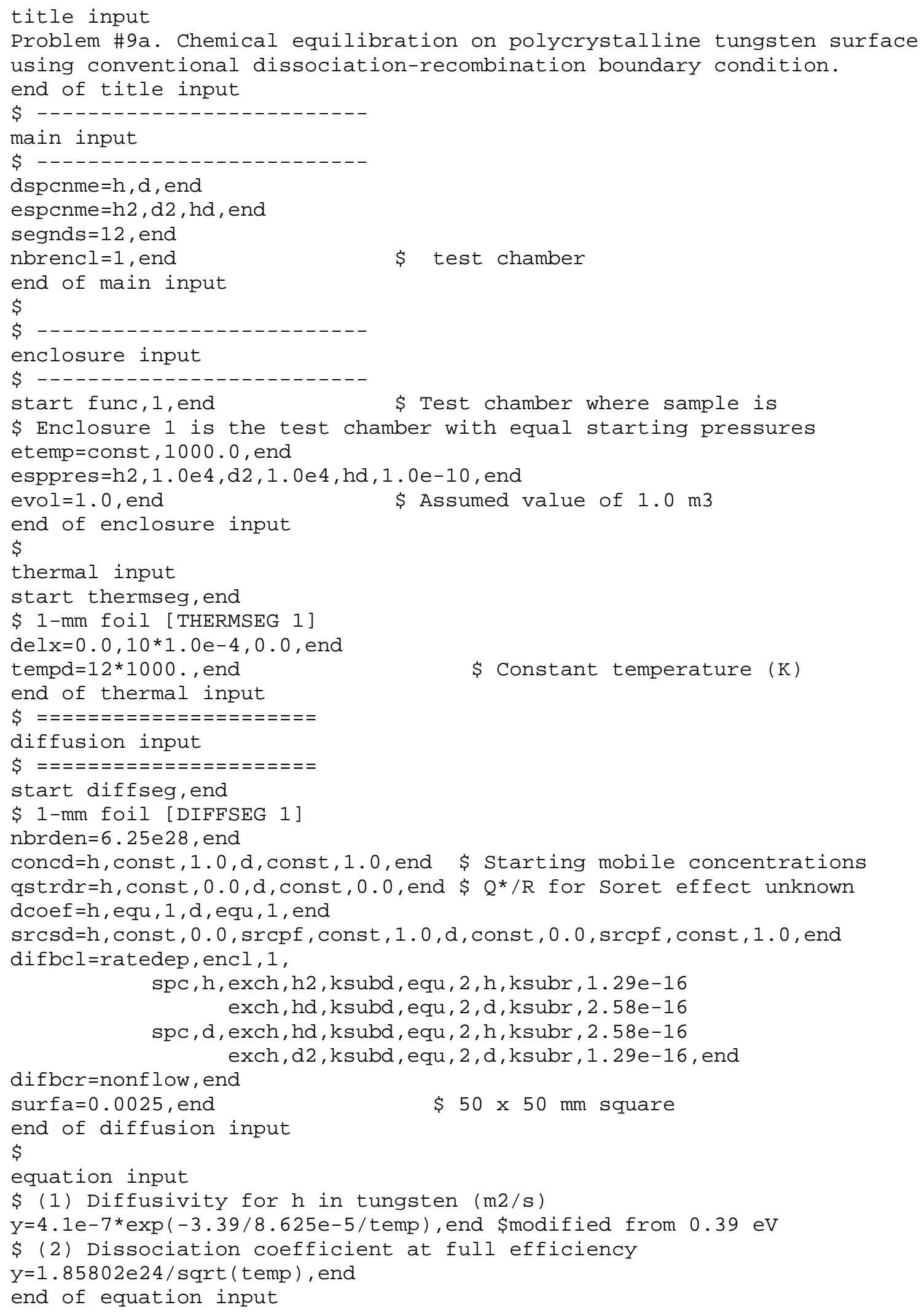


$\$$

table input

end of table input

$\$$

control input

time $=0$, end

tstep $=0.01$, end

timend $=6.1$, end

nprint $=100$, end

itermx $=15000$, end

del $c m x=1 . e-6$, end

bump $=1 . e-2$, end

bound $=2.0$, end

omega $=1.3$, end

damp $=0.7$, end

end of control input

$\$$

plot input

nplot $=20$, end

plot seg $=1$, end

plotencl=1, end

dname $=h, d$, end

ename $=\mathrm{h} 2, \mathrm{~d} 2$, hd, end

dplot=moblinv, end

eplot=press, diff, end

end of plot input

$\$$

end of data
\$ makes plotfile entry every 0.2 sec

$\$$ segments for which plot info is needed

$\$$ enclosures for which plot info is needed

\$ diffusing species for which plot info is needed

$\$$ enclosure species for which plot info is needed 
Species Equilibration on a Reactive Surface (Val-1ib, see p. 39)

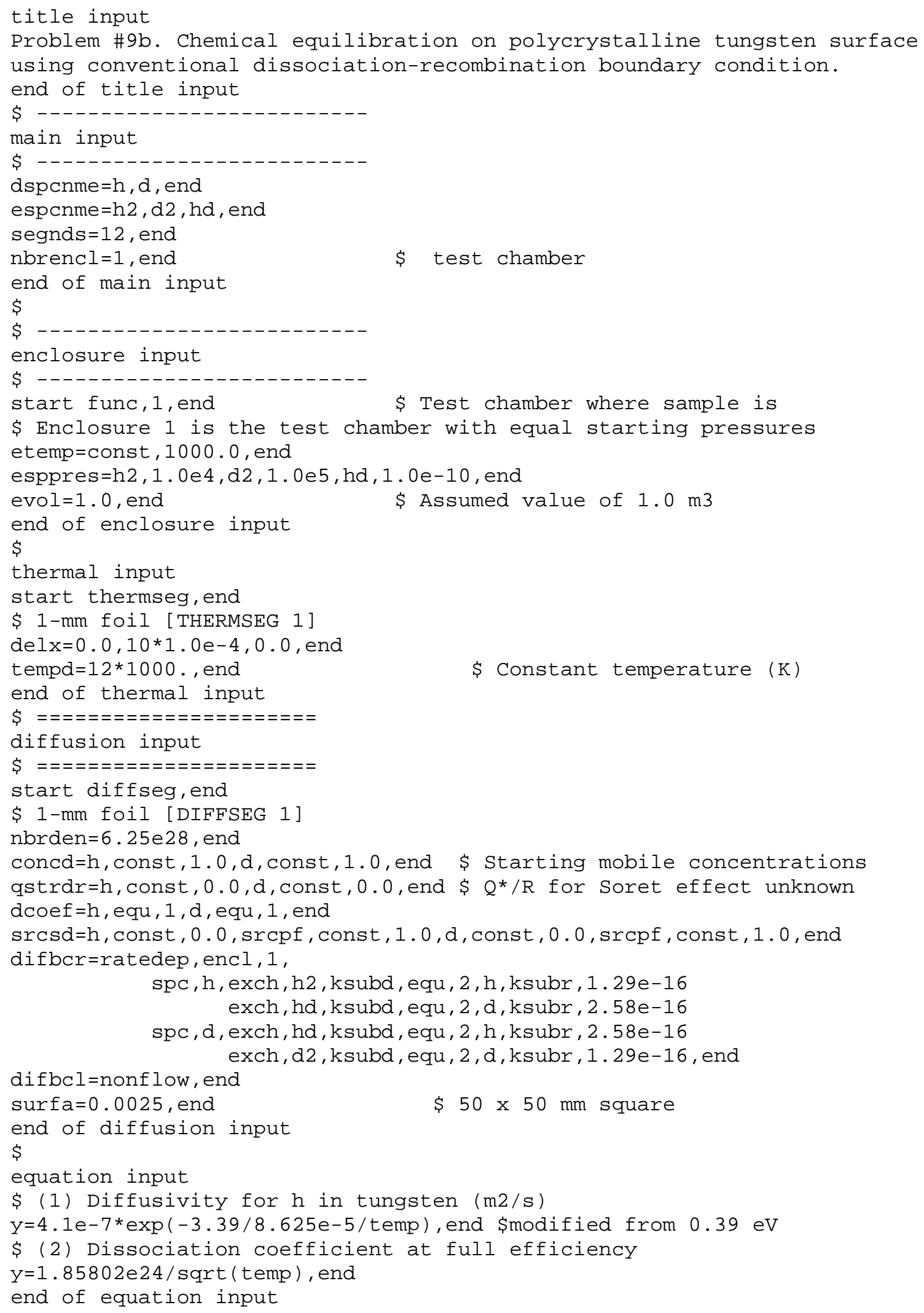


$\$$

table input

end of table input

$\$$

control input

time $=0$, end

tstep $=0.01$, end

timend $=6.1$, end

nprint $=100$, end

itermx $=1500$, end

del $c m x=1 . e-6$, end

bump $=1 . e-2$, end

bound $=2.0$, end

omega $=1.3$, end

damp $=0.7$, end

end of control input

$\$$

plot input

nplot $=20$, end

plot seg $=1$, end

plotencl=1, end

dname $=h, d$, end

ename $=\mathrm{h} 2, \mathrm{~d} 2$, hd, end

dplot=moblinv, end

eplot=press, diff, end

end of plot input

$\$$

end of data
\$ makes plotfile entry every 0.2 sec

$\$$ segments for which plot info is needed

$\$$ enclosures for which plot info is needed

\$ diffusing species for which plot info is needed

$\$$ enclosure species for which plot info is needed 
Species Equilibration on a Reactive Surface (Val-1ic, see p. 39)

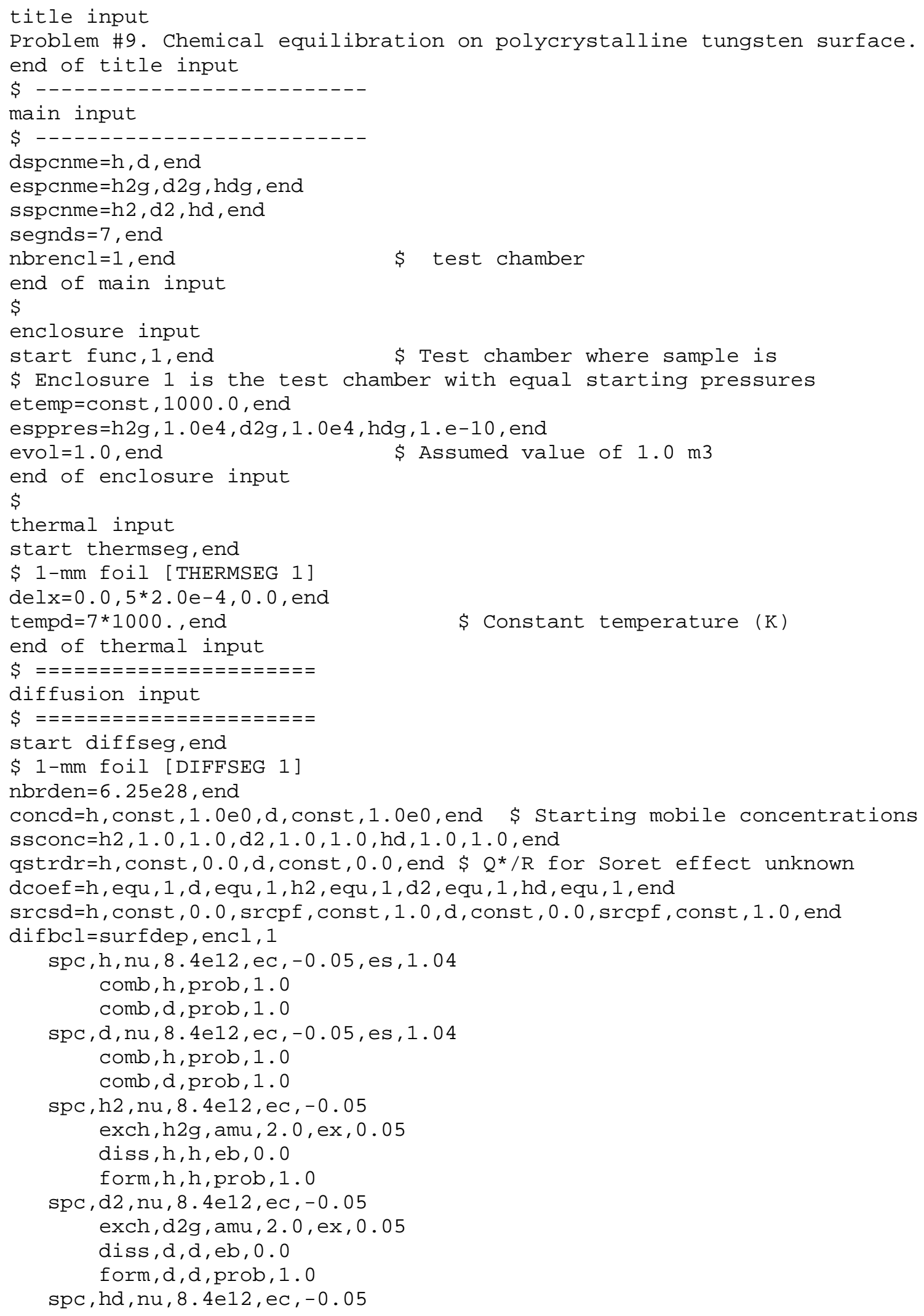


exch, hdg, amu, 2.0, ex, 0.05

diss, h, d, eb, 0.0

form, h, d, prob, 1.0 , end

difbcr=nonflow, end

surfa $=0.0025$, end $\quad \$ 50 \times 50 \mathrm{~mm}$ square

end of diffusion input

\$

equation input

$\$$ (1) Diffusivity for $h$, d in tungsten (m2/s)

$y=5.33 e-7 * \exp (-0.39 / 8.625 e-5 /$ temp $)$, end

end of equation input

$\$$

table input

end of table input

$\$$

control input

time $=0$, end

tstep $=0.01$, end

timend $=10$, end

nprint $=1$, end

itermx $=1000$, end

delcmx $=1 . e-7$, end

bump $=1 . e-4$, end

bound $=10$, , end

omega $=1.0$, end

damp $=0.7$

end of control input

$\$$

plot input

nplot $=50$, end

$\$$ makes plotfile entry every $0.2 \mathrm{sec}$

plot seg $=1$, end

$\$$ segments for which plot info is needed

plotencl $=1$, end

$\$$ enclosures for which plot info is needed

dname $=h, d$, end

$\$$ diffusing species for which plot info is needed

sname $=\mathrm{h} 2, \mathrm{~d} 2$, hd, end

$\$$ surface species for which plot info is needed

ename=h2g, d2g, hdg, end $\$$ enclosure species for which plot info is needed

dplot=moblinv, end

eplot=press, diff, end

end of plot input

$\$$

end of data 
Species Equilibration on a Reactive Surface (Val-1id, see p. 39)

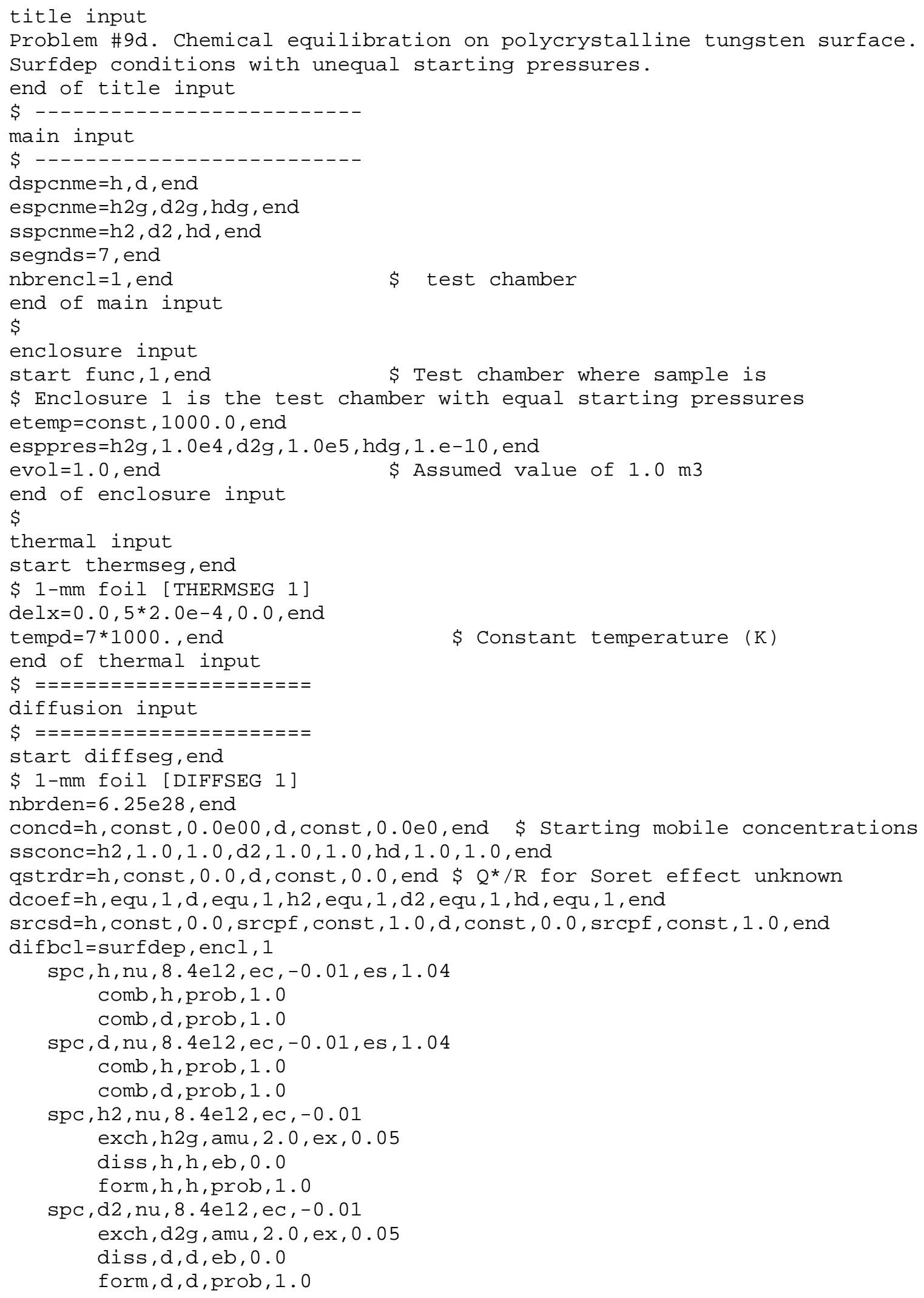




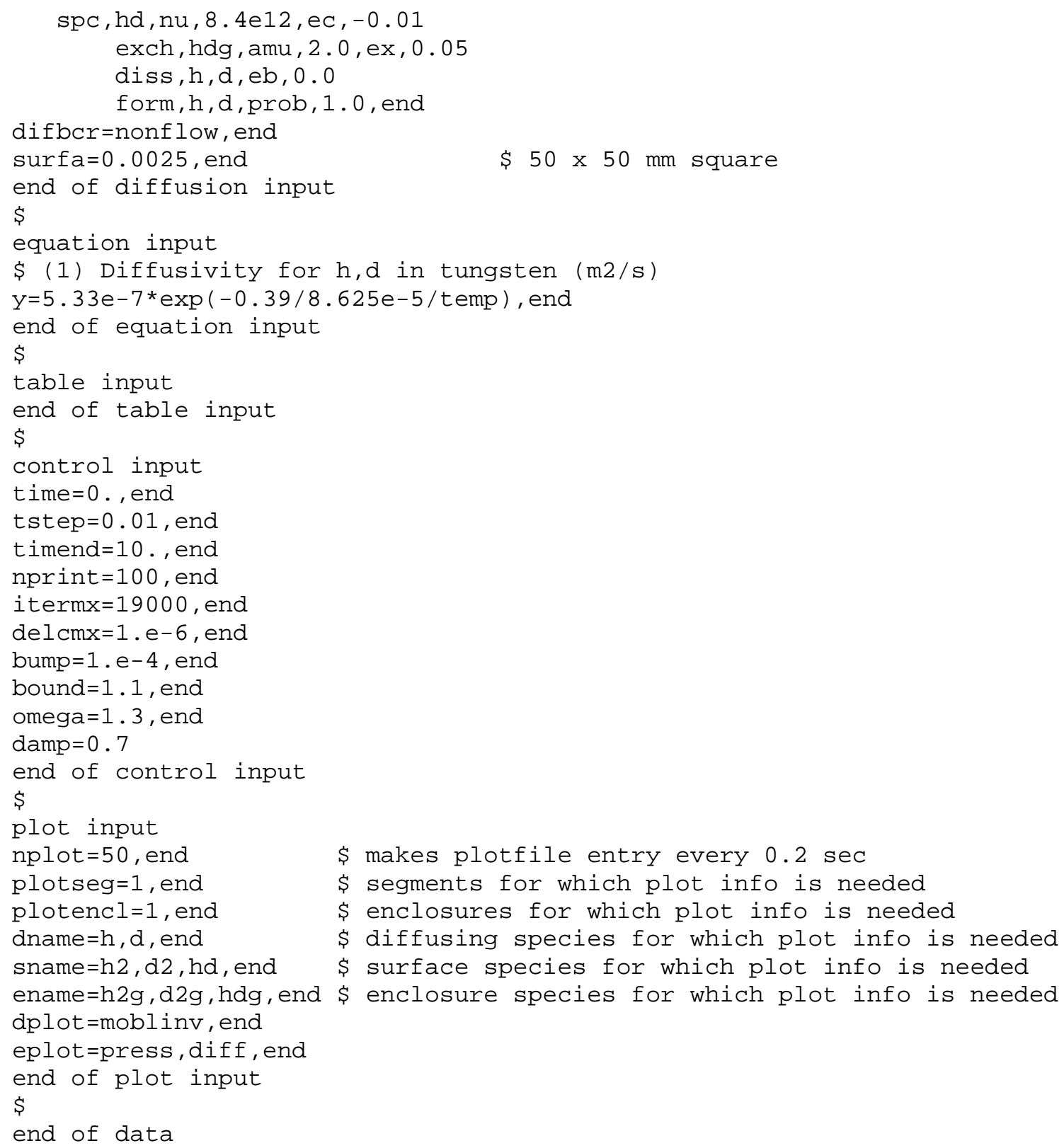


Problem 1ja: Radioactive Decay of Mobile Tritium in a Slab (Val-1ja, see p. 41)

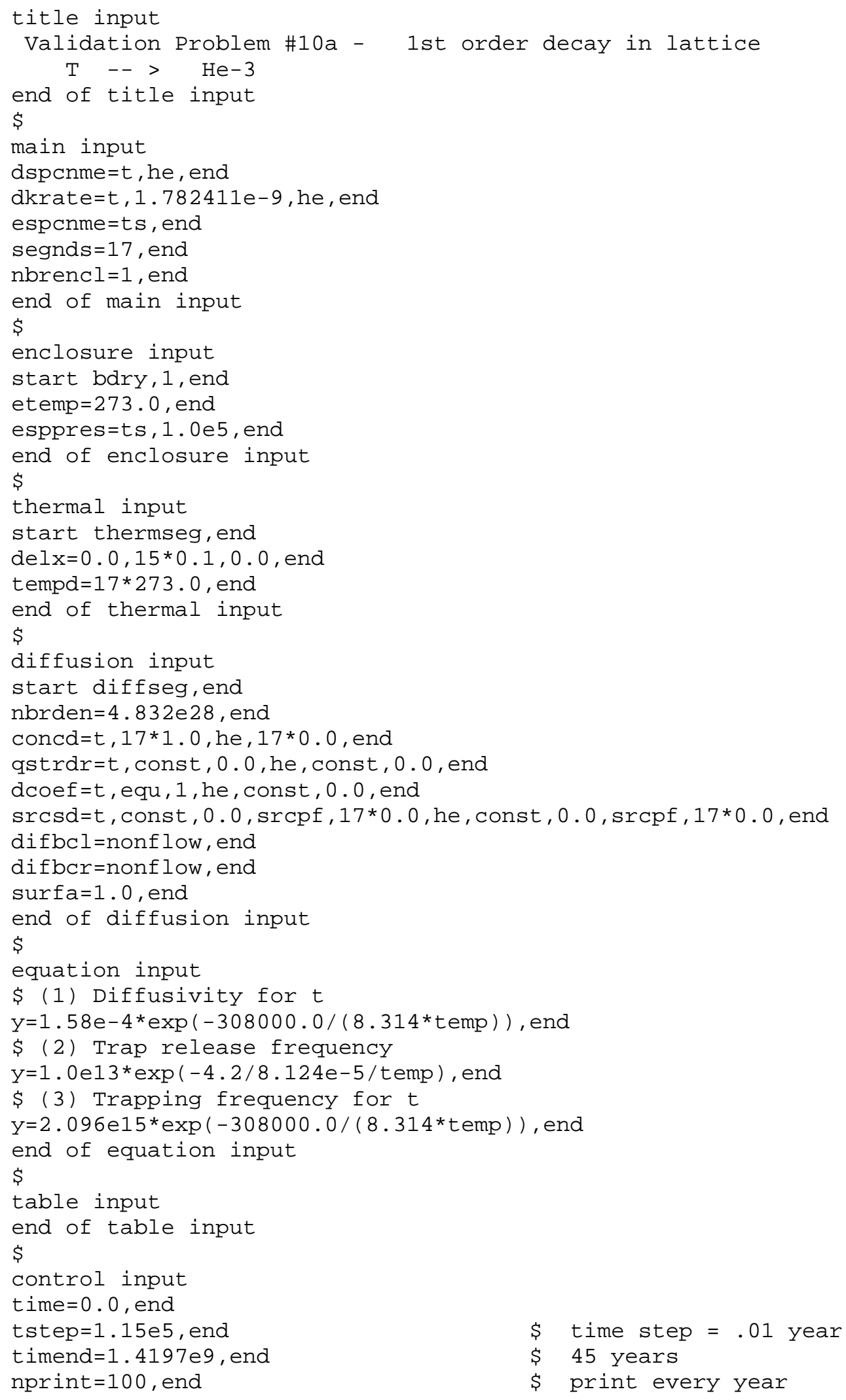




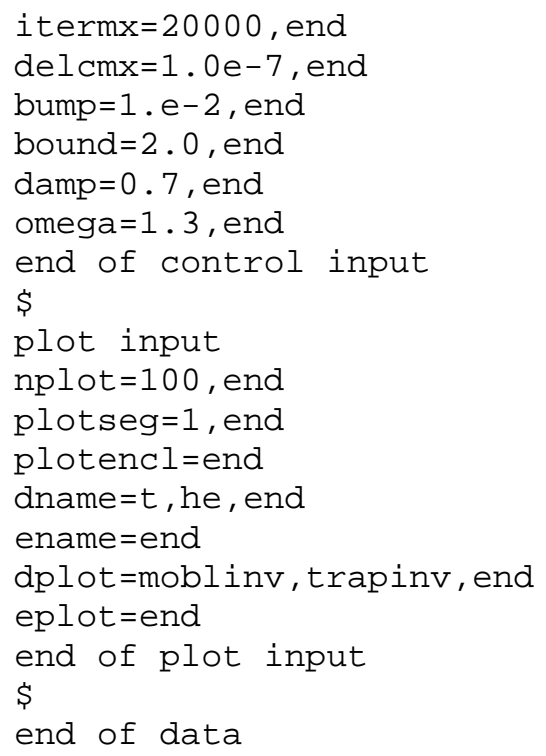


Problem 1jb: Decay of Tritium in a Distributed Trap (Val-1jb, see p. 43)

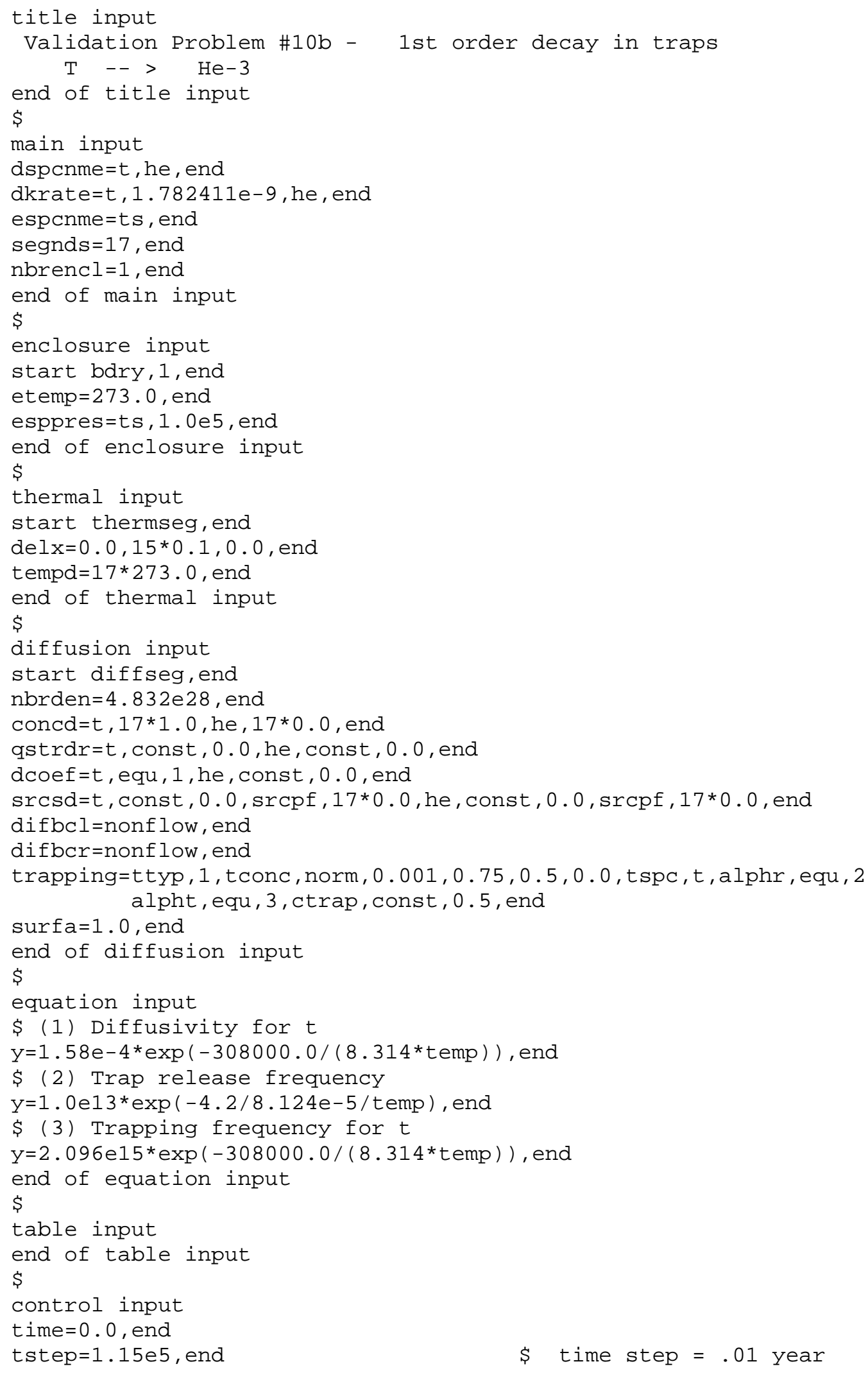




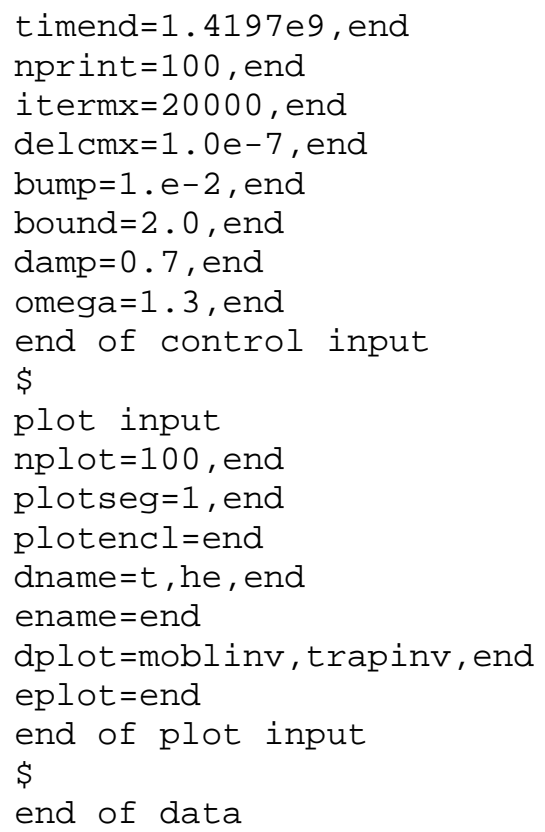

\$ 45 years

\$ print every year
\$ makes plotfile entry every $1 / 10$ year
$\$$ segments for which plot info is needed
$\$$ enclosure info is not needed
$\$$ diffusing species for which plot info is needed
\$ enclosure species for which plot info is needed 
Ion Implantation Experiment (Val-2a, see p. 45)

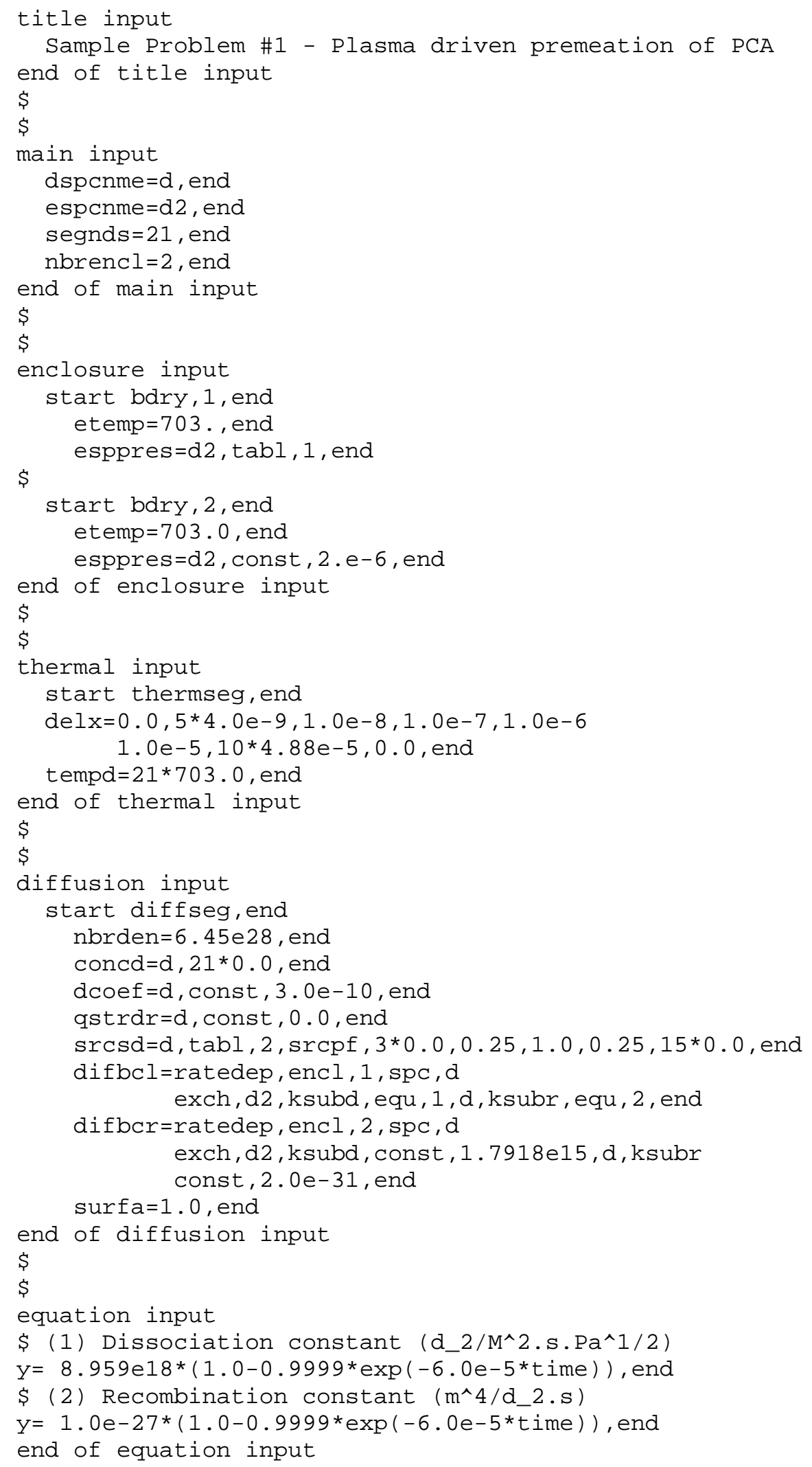




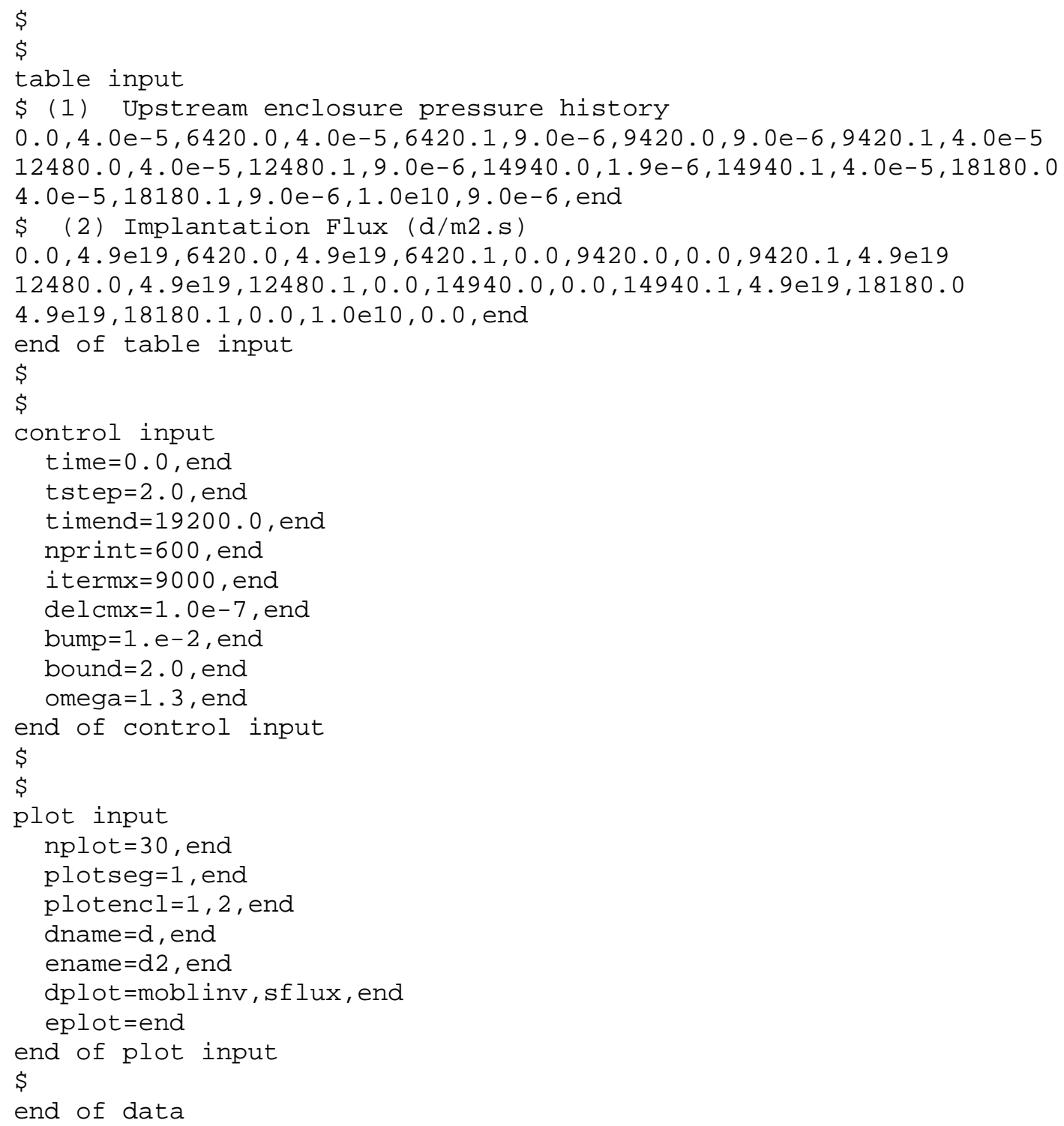


Diffusion Experiment in Beryllium (Val-2ba, Val-2bb, see p. 46)

\section{Charging Segment}

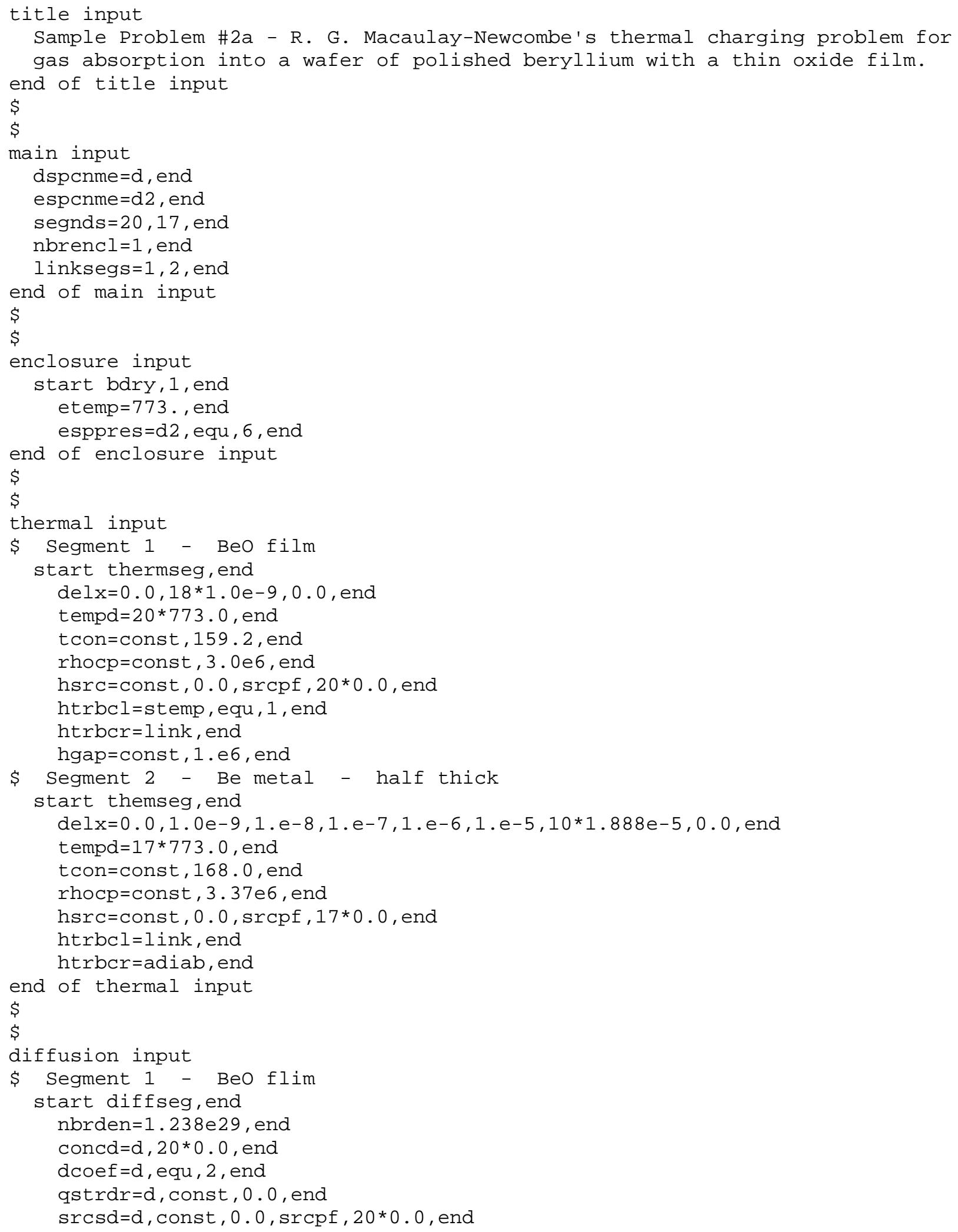




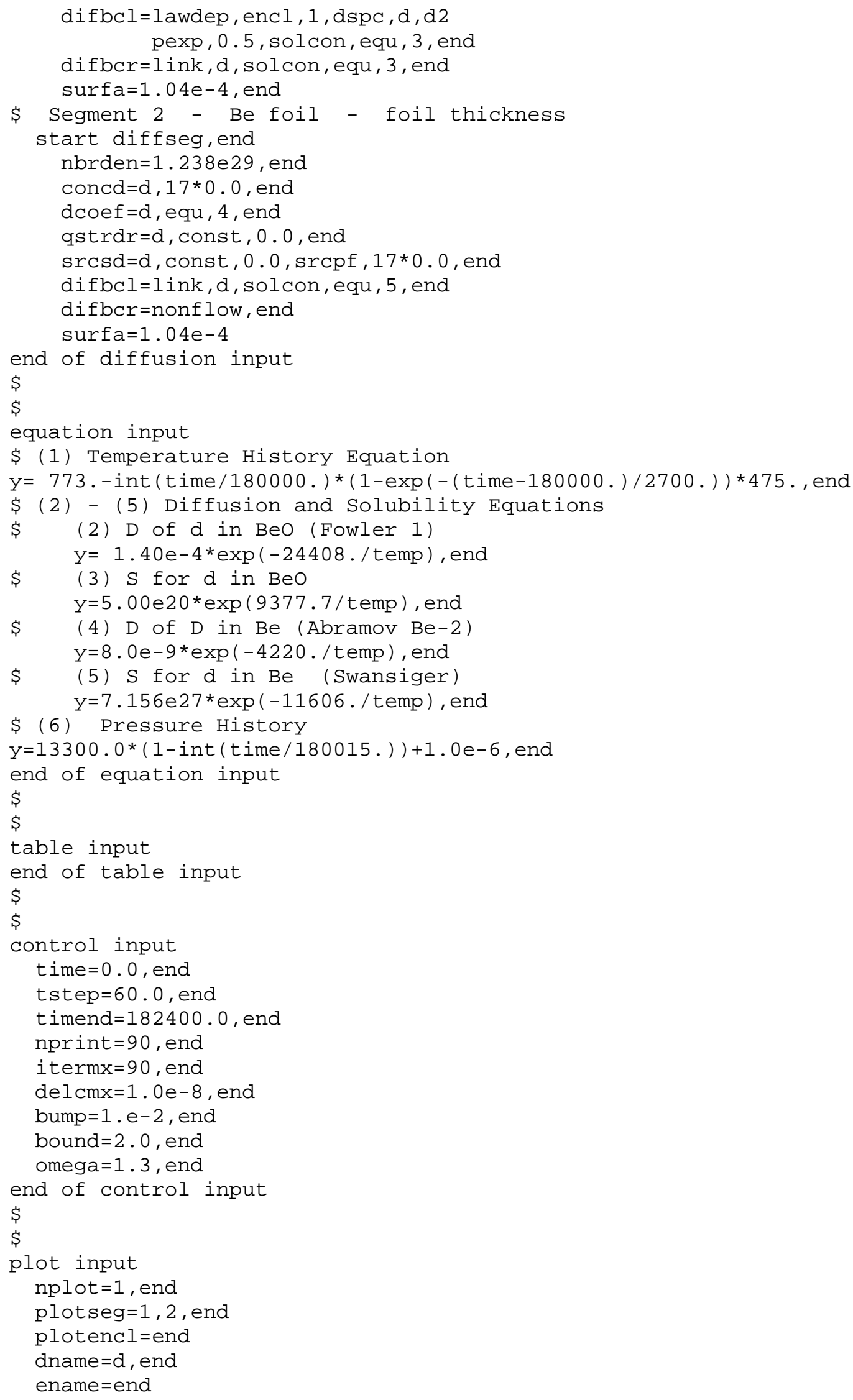




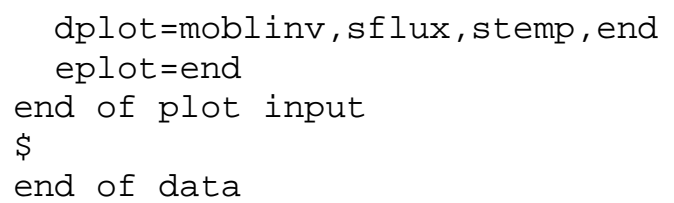

\section{Desorption Segment (Restart)}

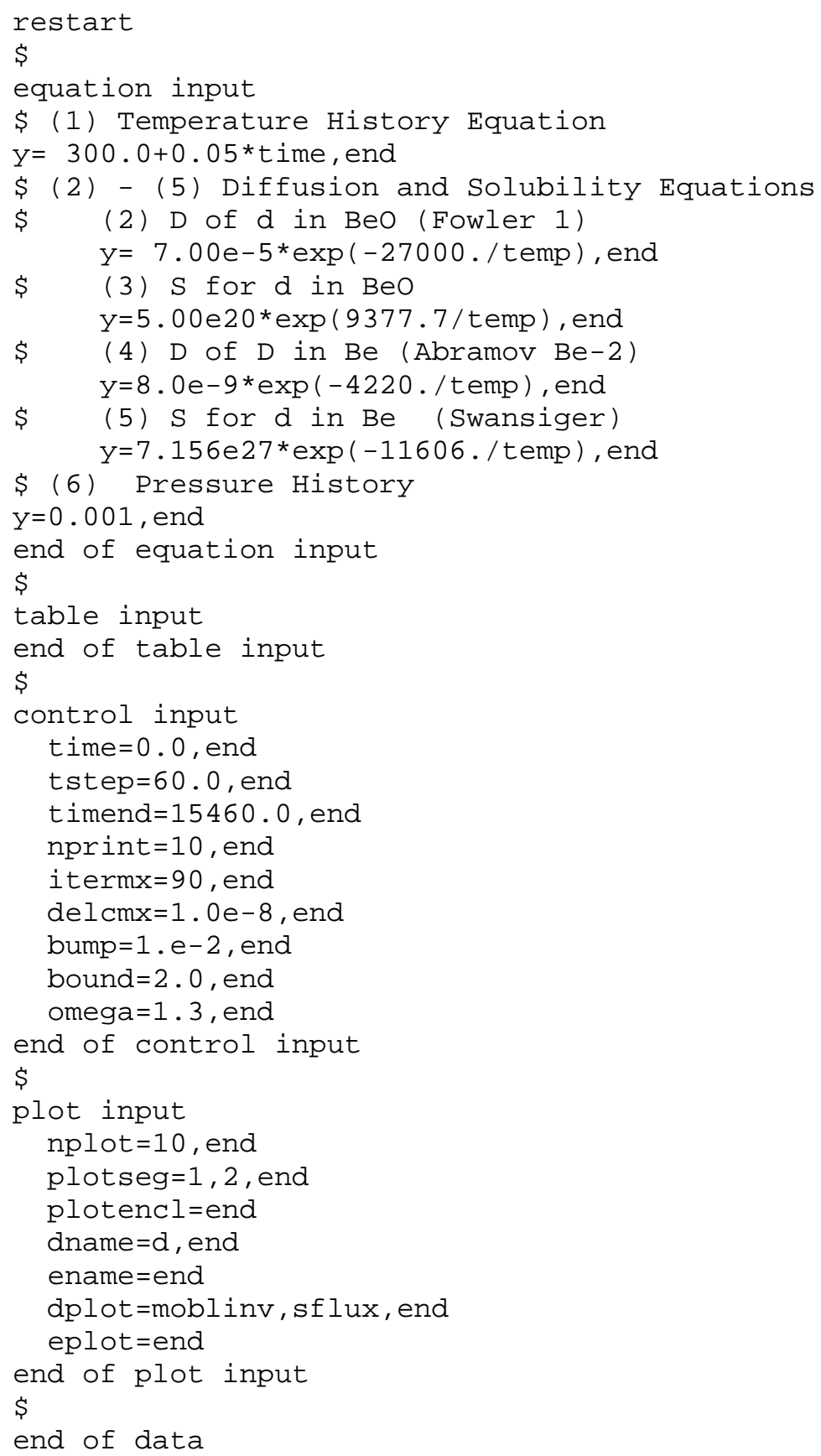


Test Cell Release Experiment (Val-2c, see p. 48)

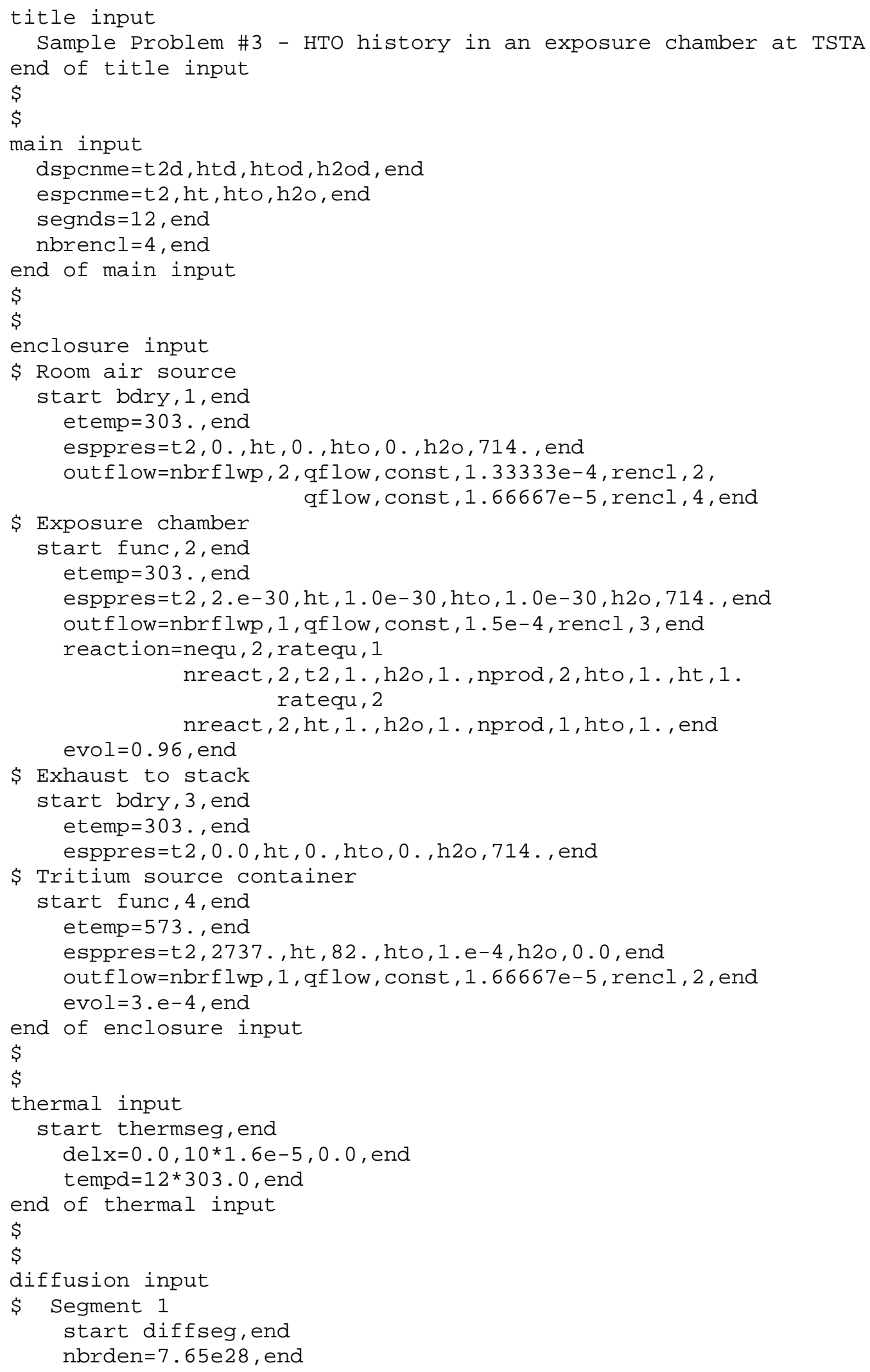




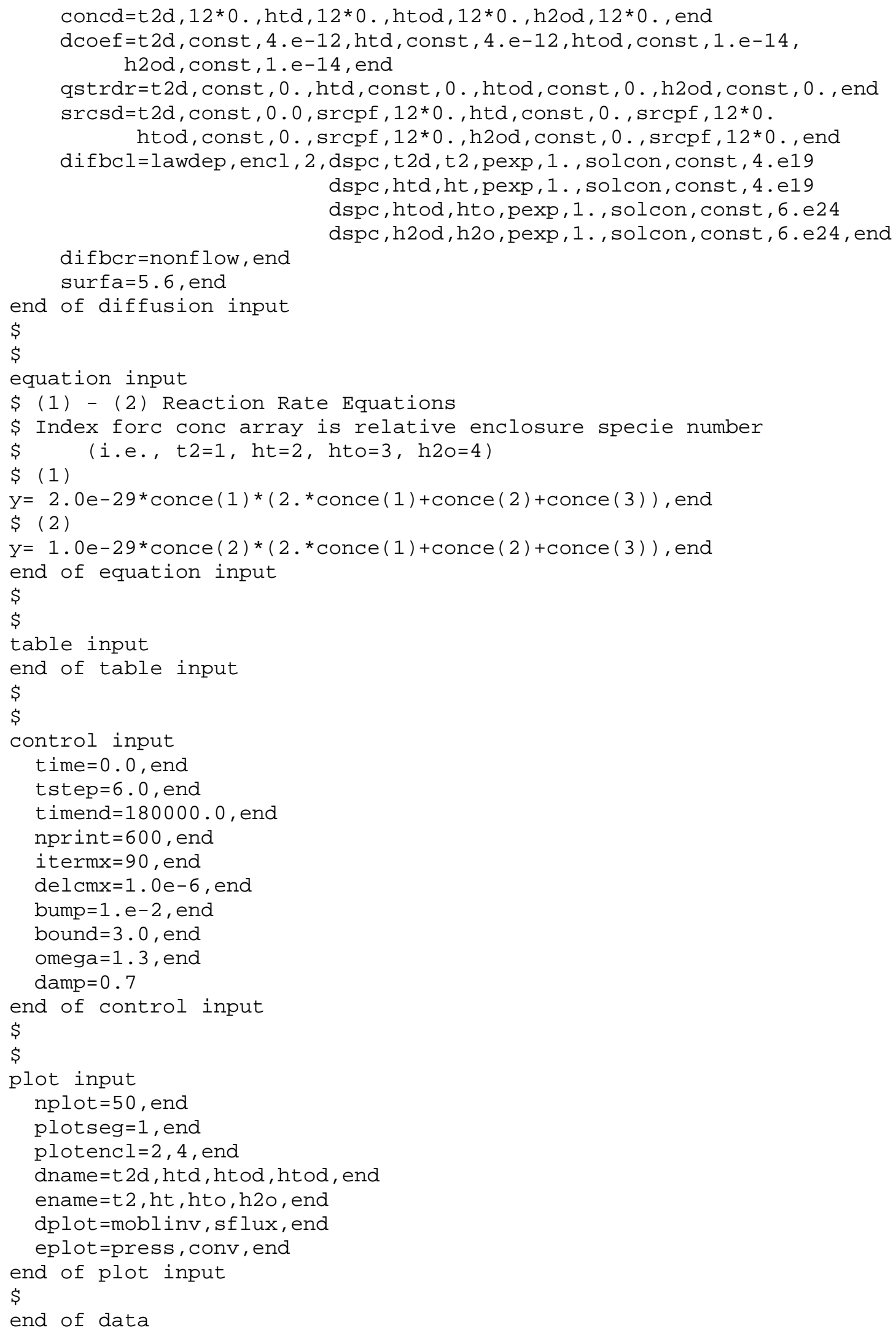


Problem 2d. Thermal Desorption Spectroscopy on Tungsten (Val-2d, see p. 49)

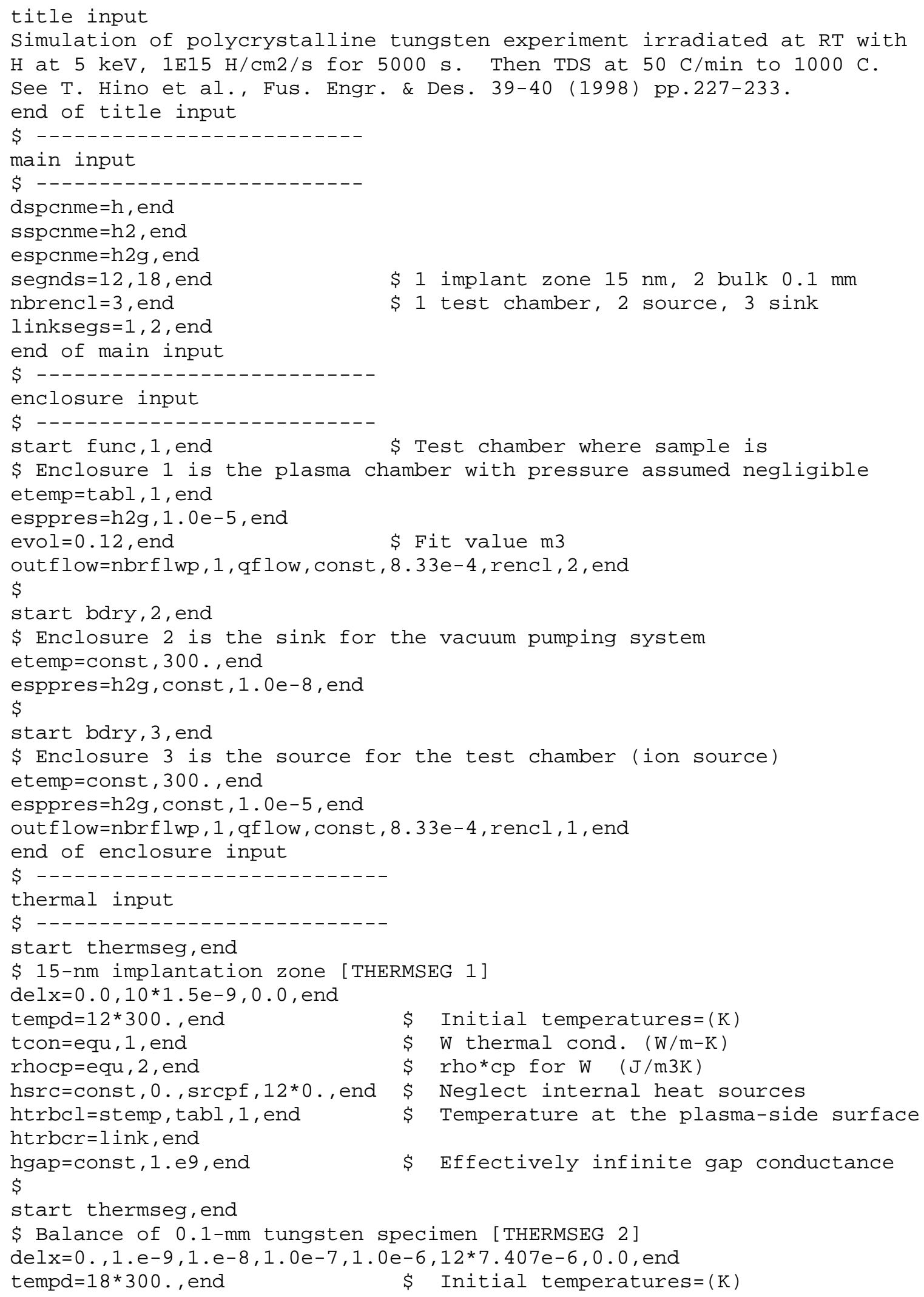




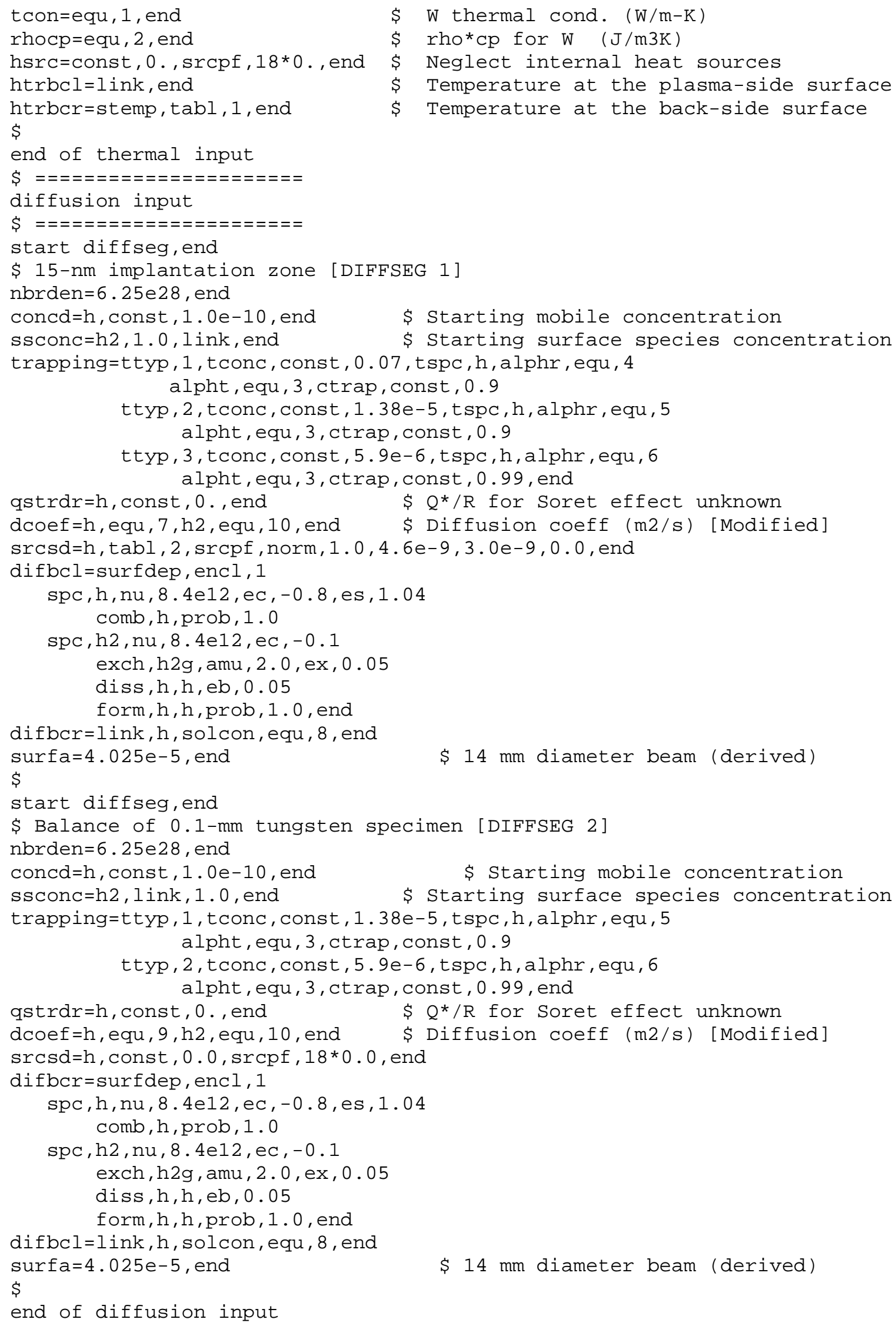




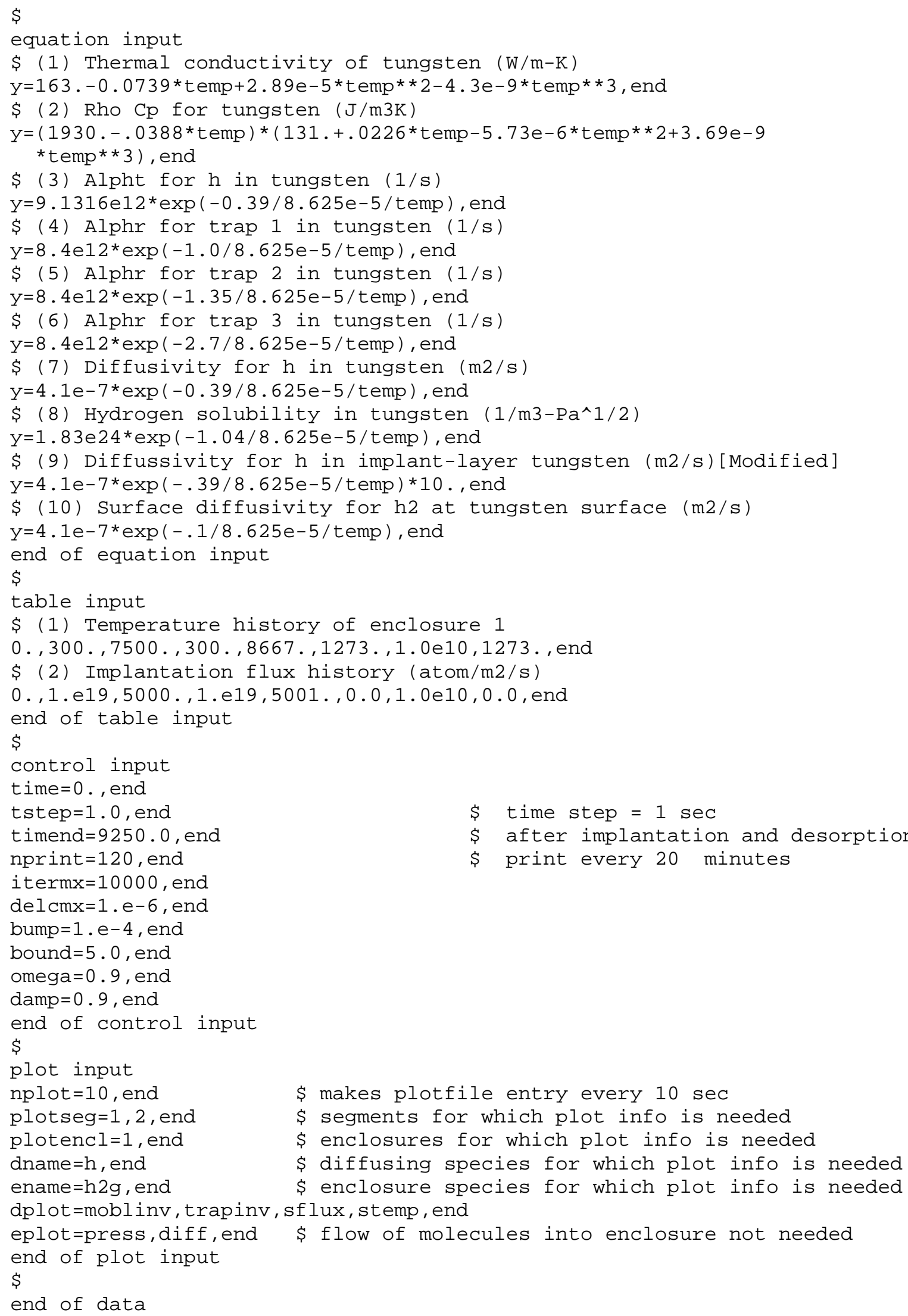


Co-permeation of H and D through Pd (Val-2ea, see p. 53)

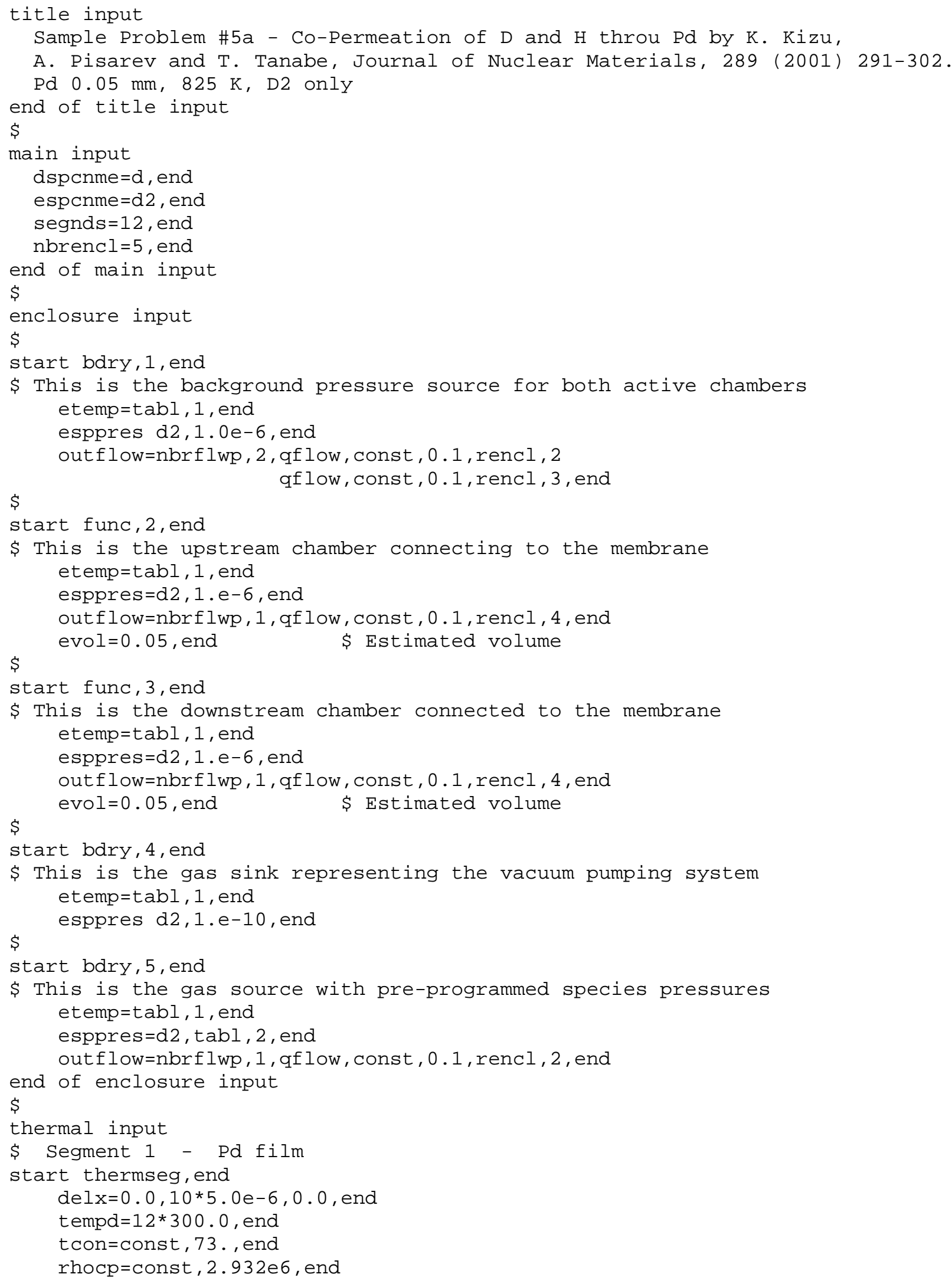




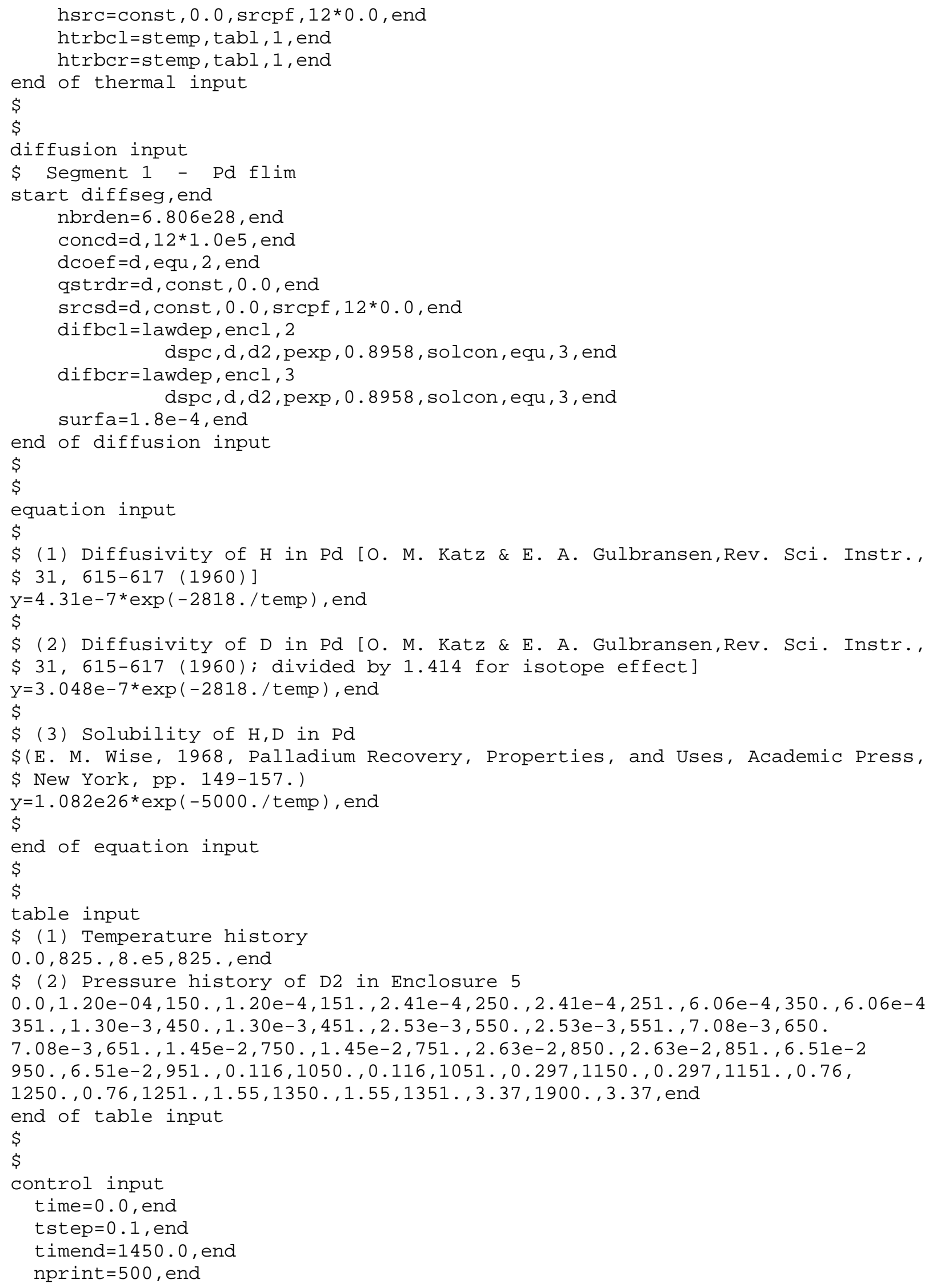




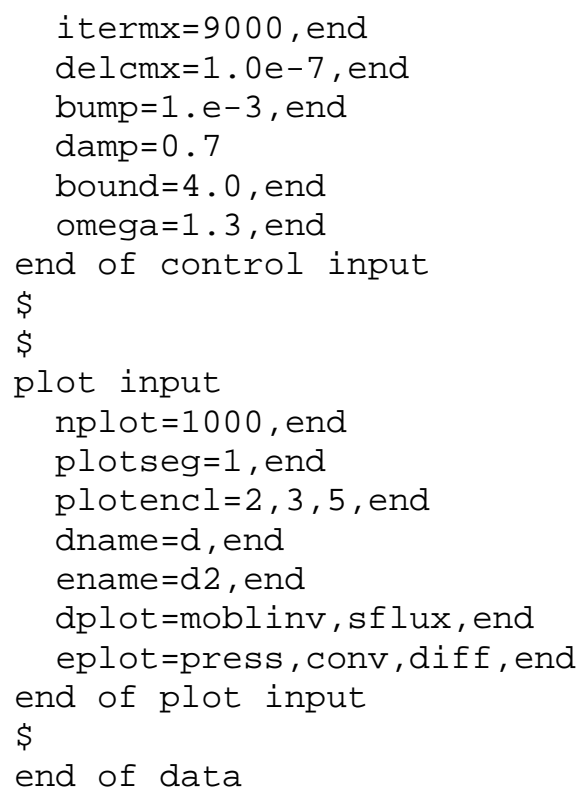


Co-permeation of H and D through Pd (Val-2eb, see p. 53)

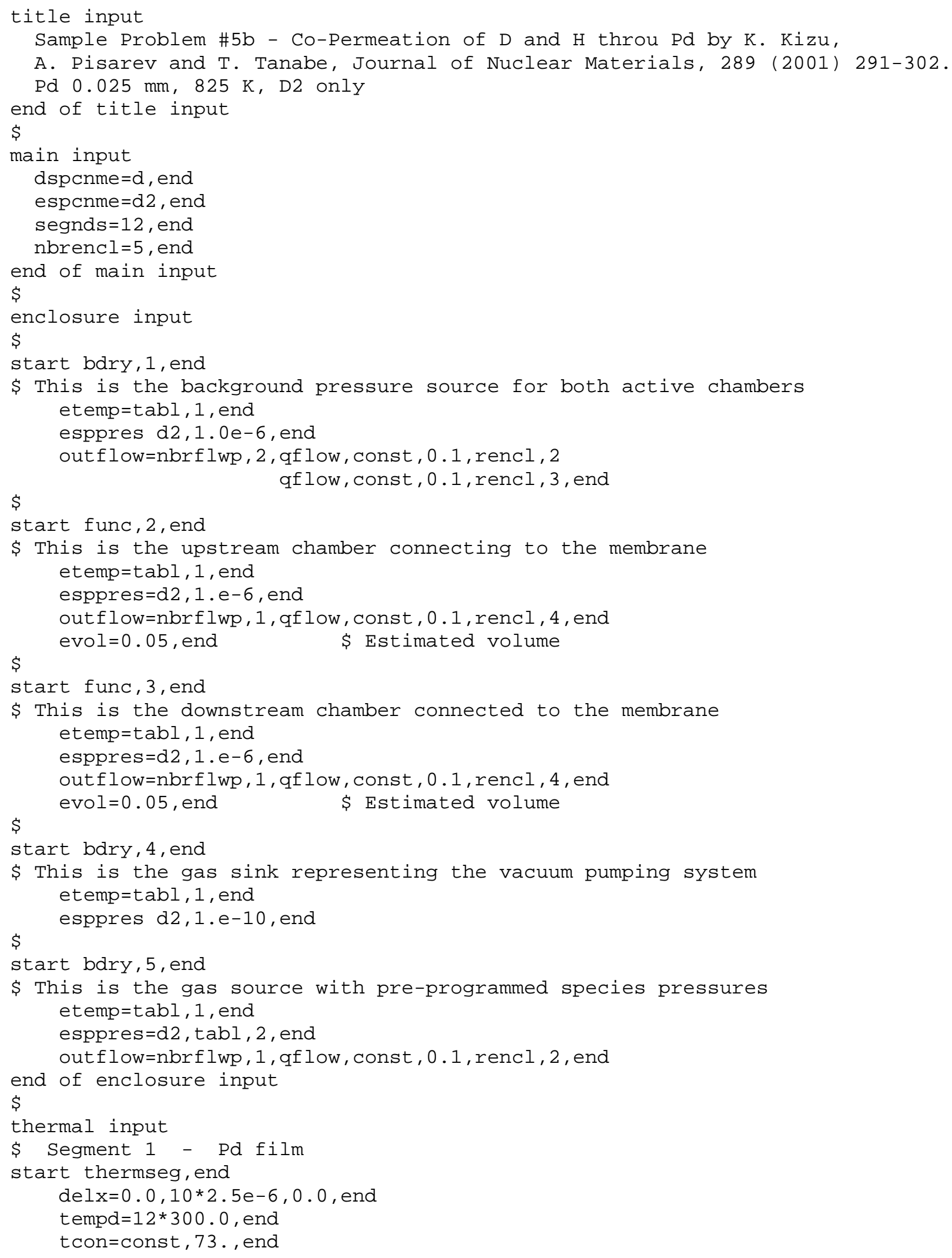




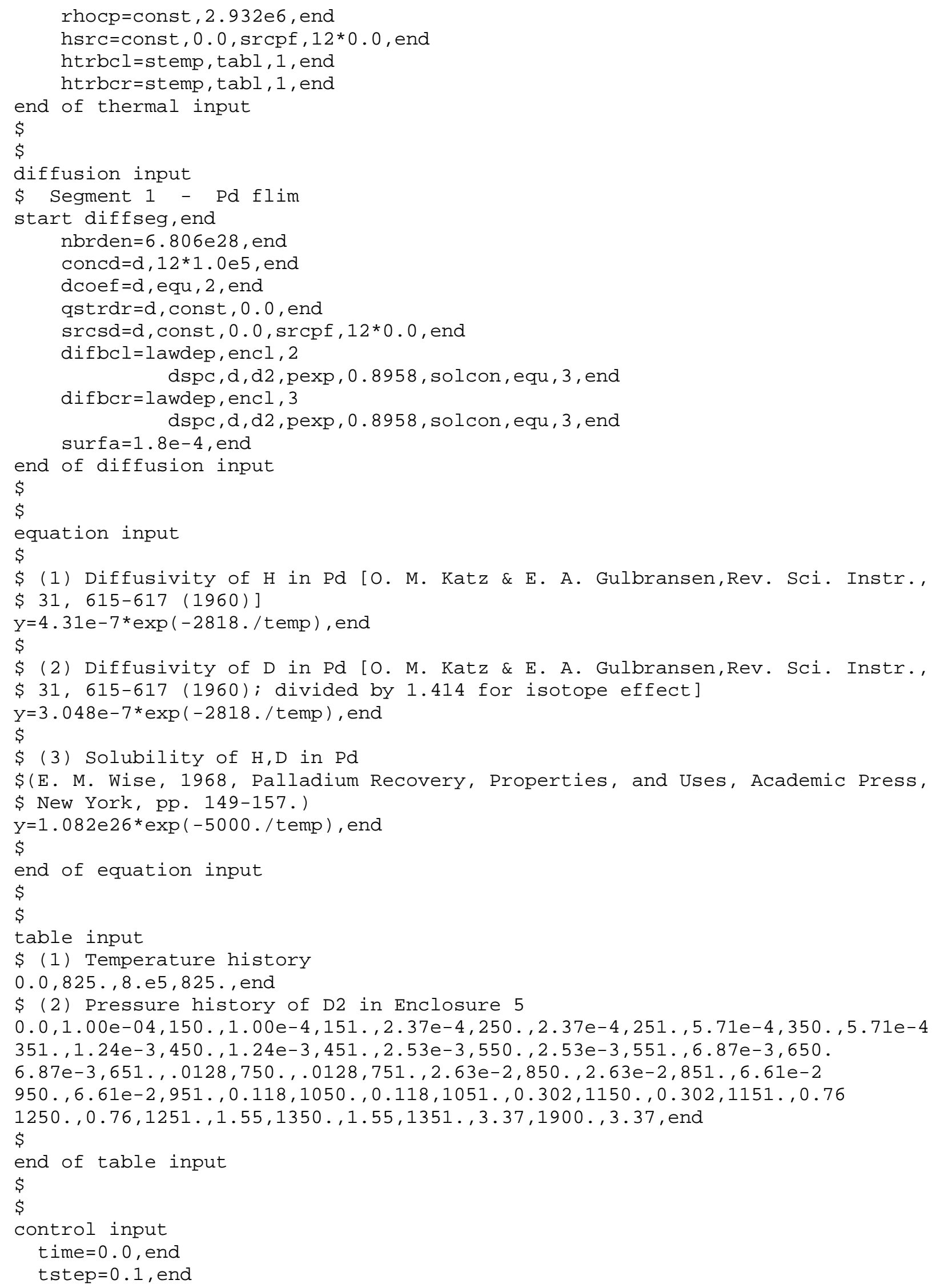




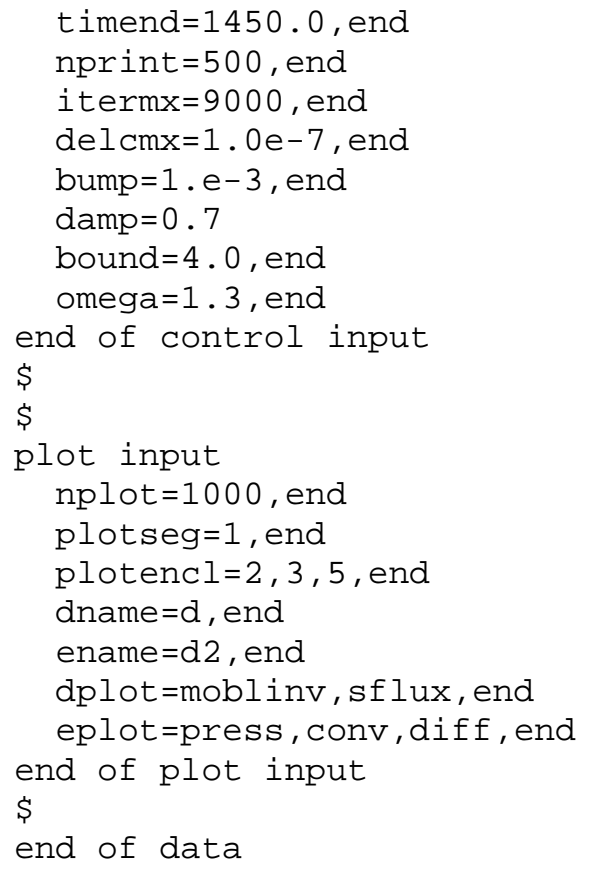


Co-permeation of H and D through Pd (Val-2ec, see p. 53)

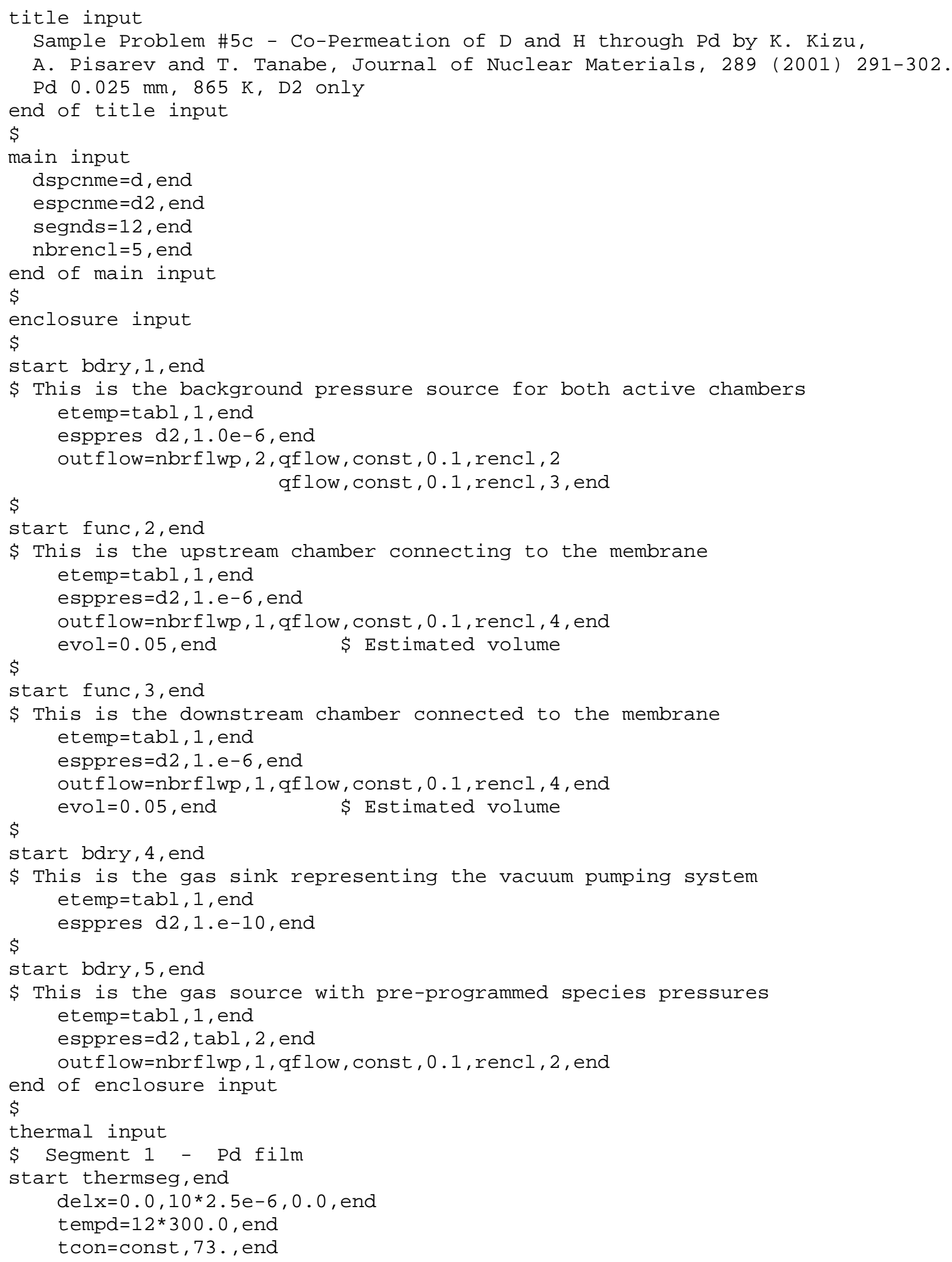




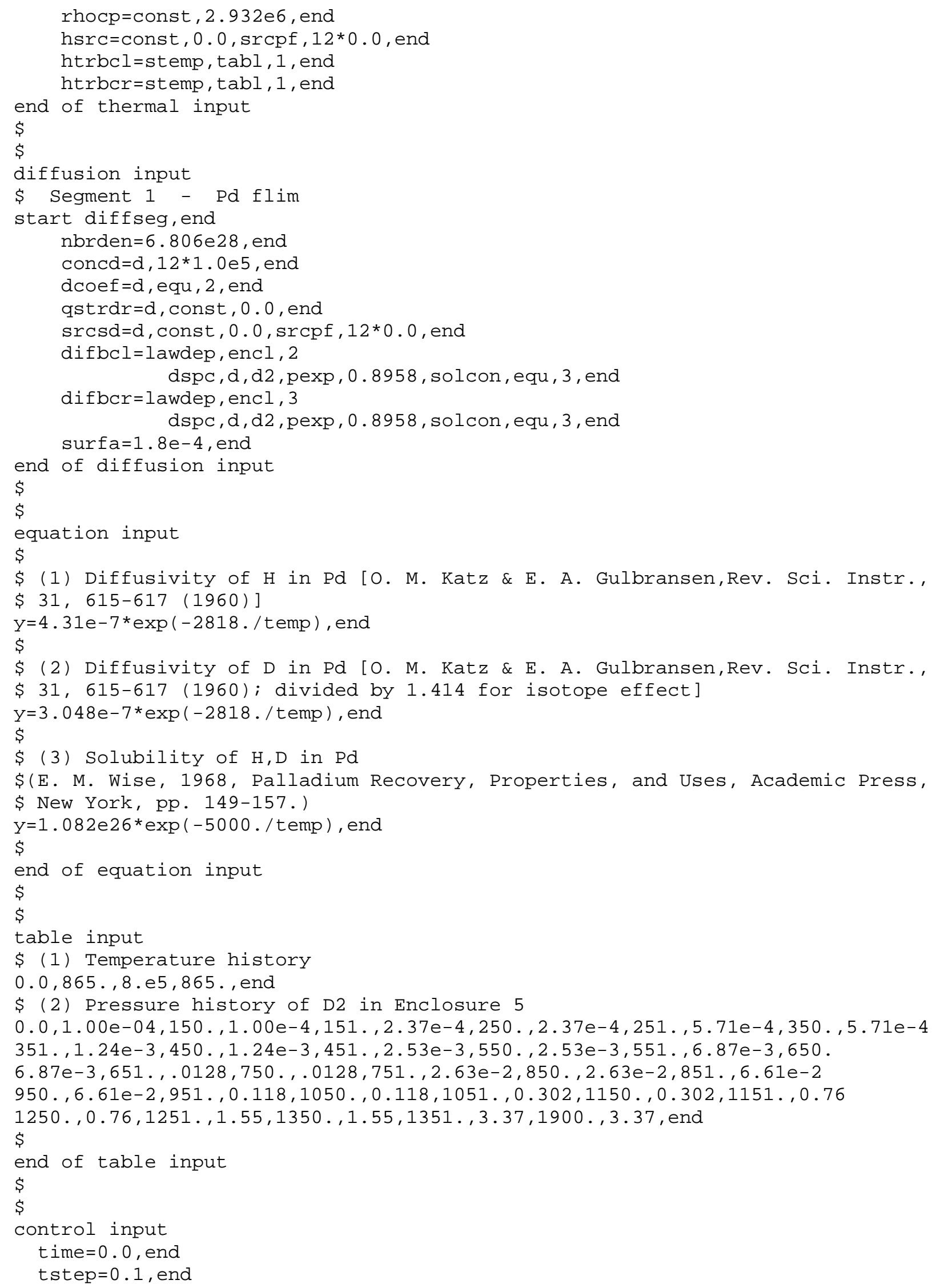




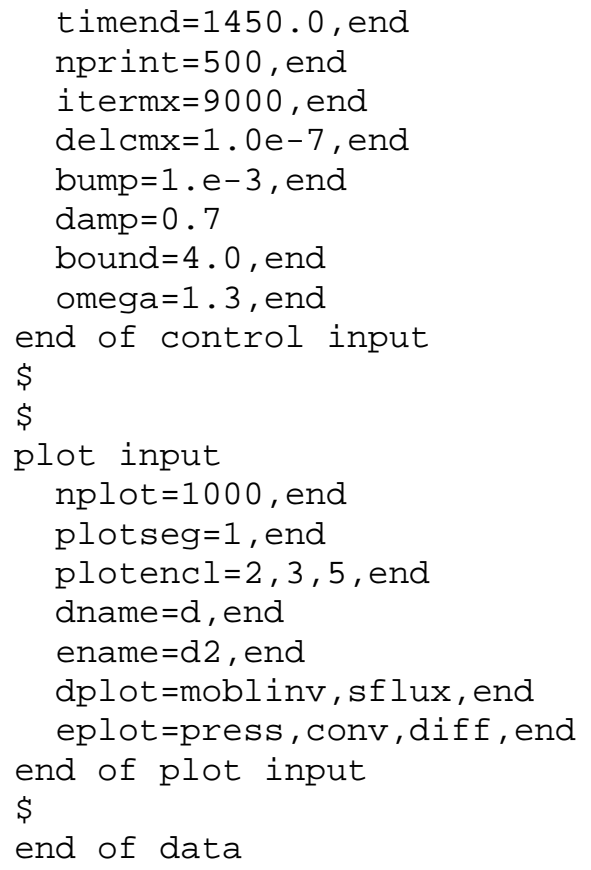


Co-permeation of H and D through Pd (Val-2ed, see p. 53)

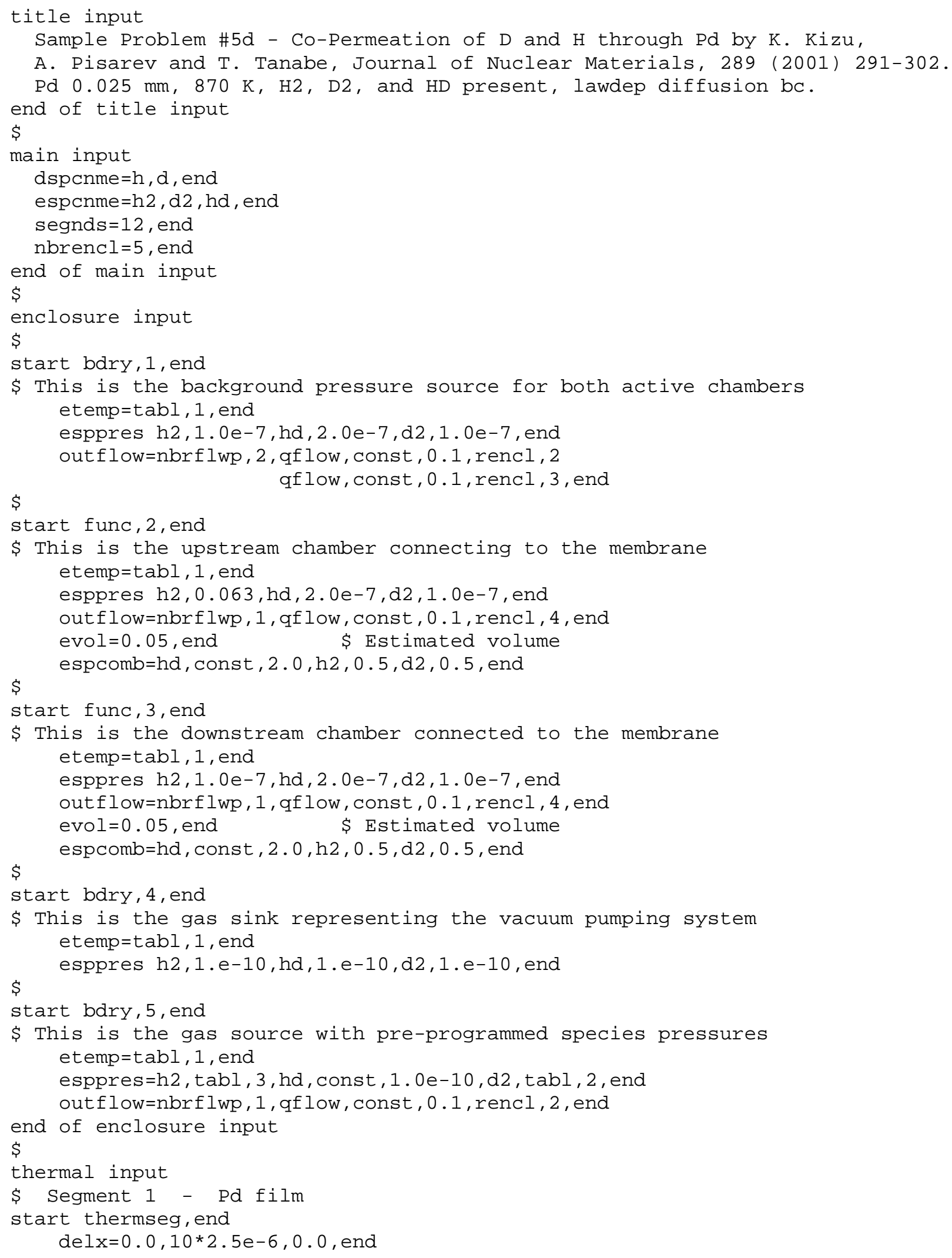




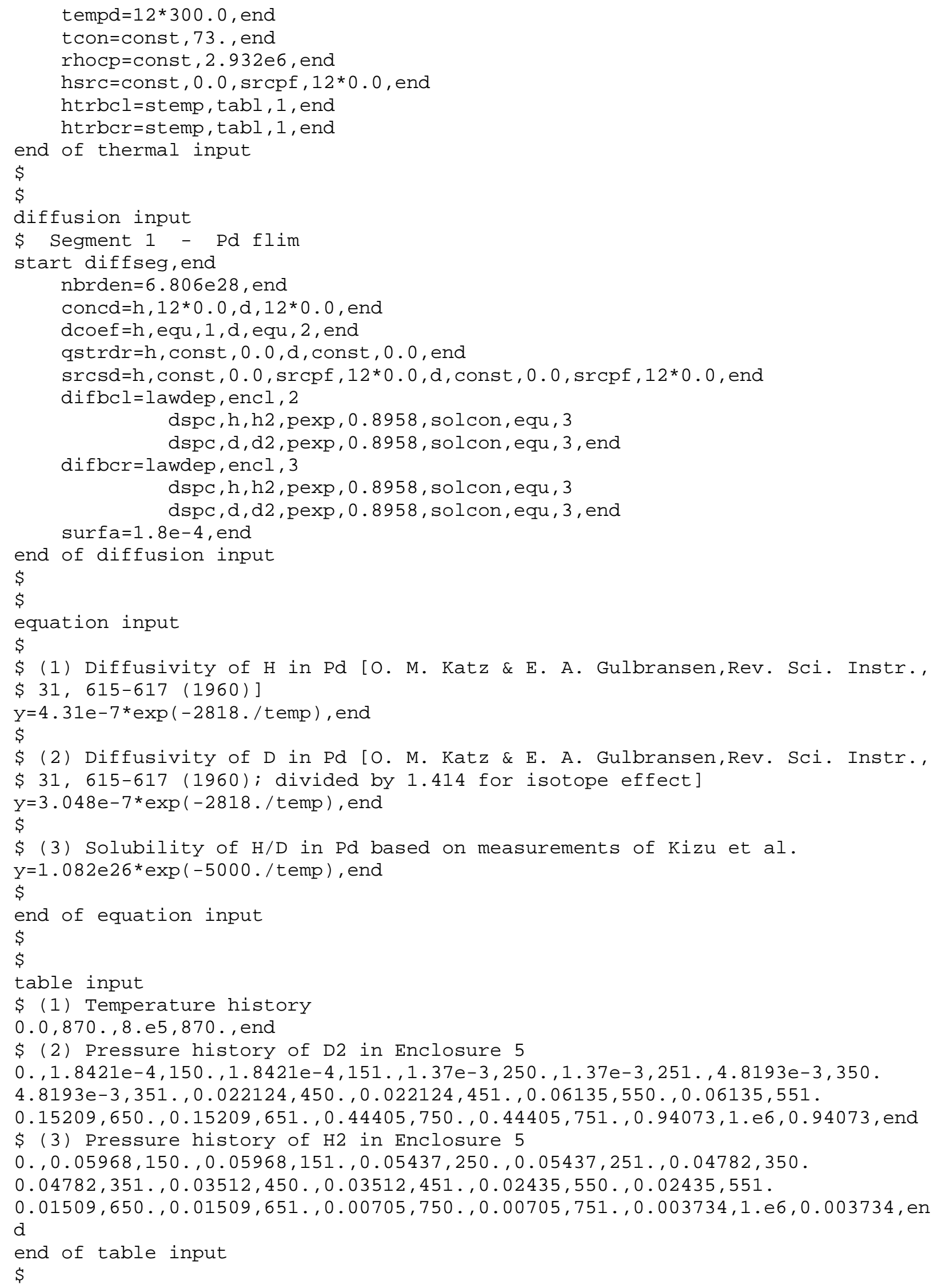




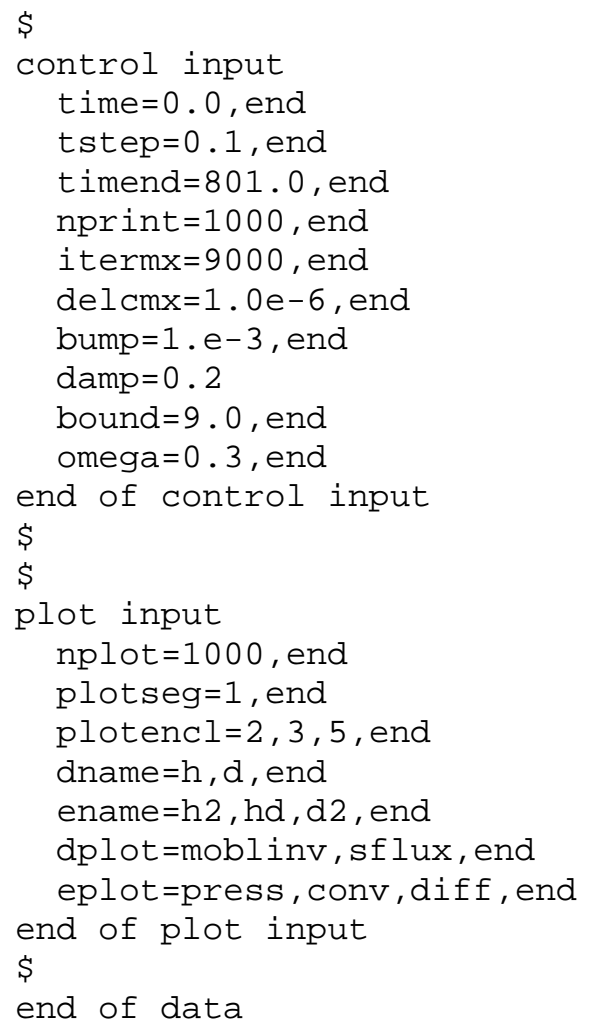


Co-permeation of H and D through Pd (Val-2eea, see p. 53)

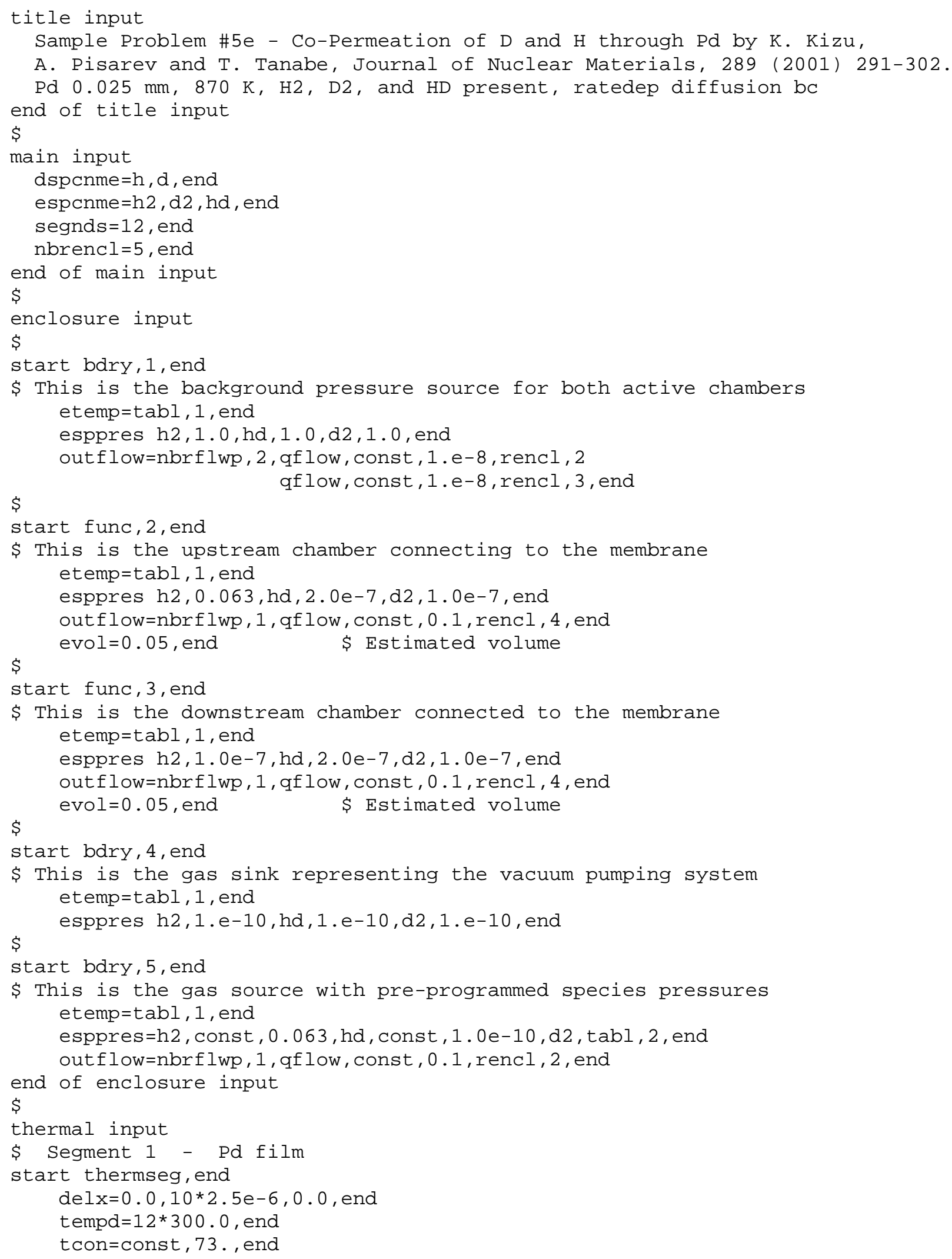




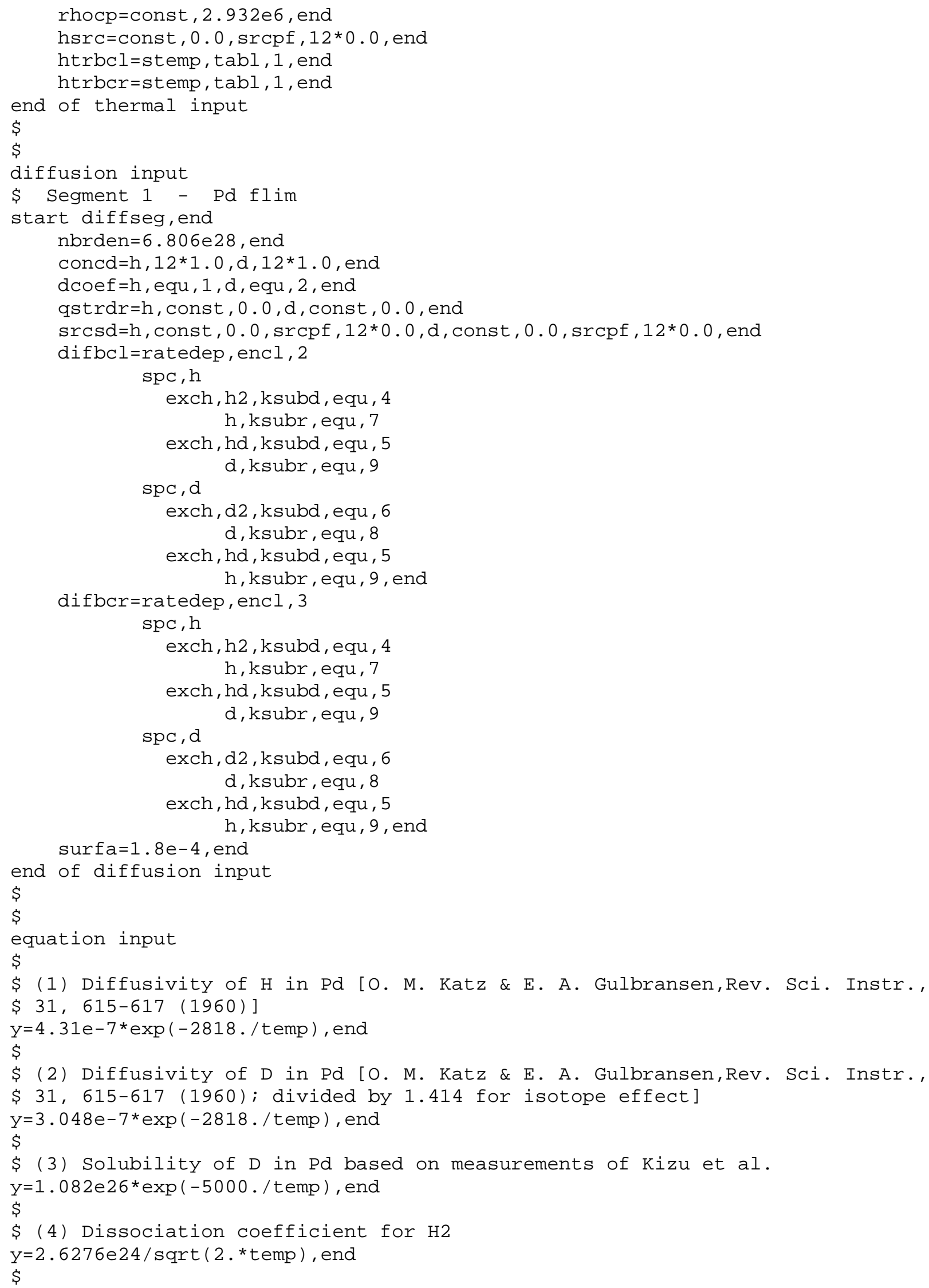




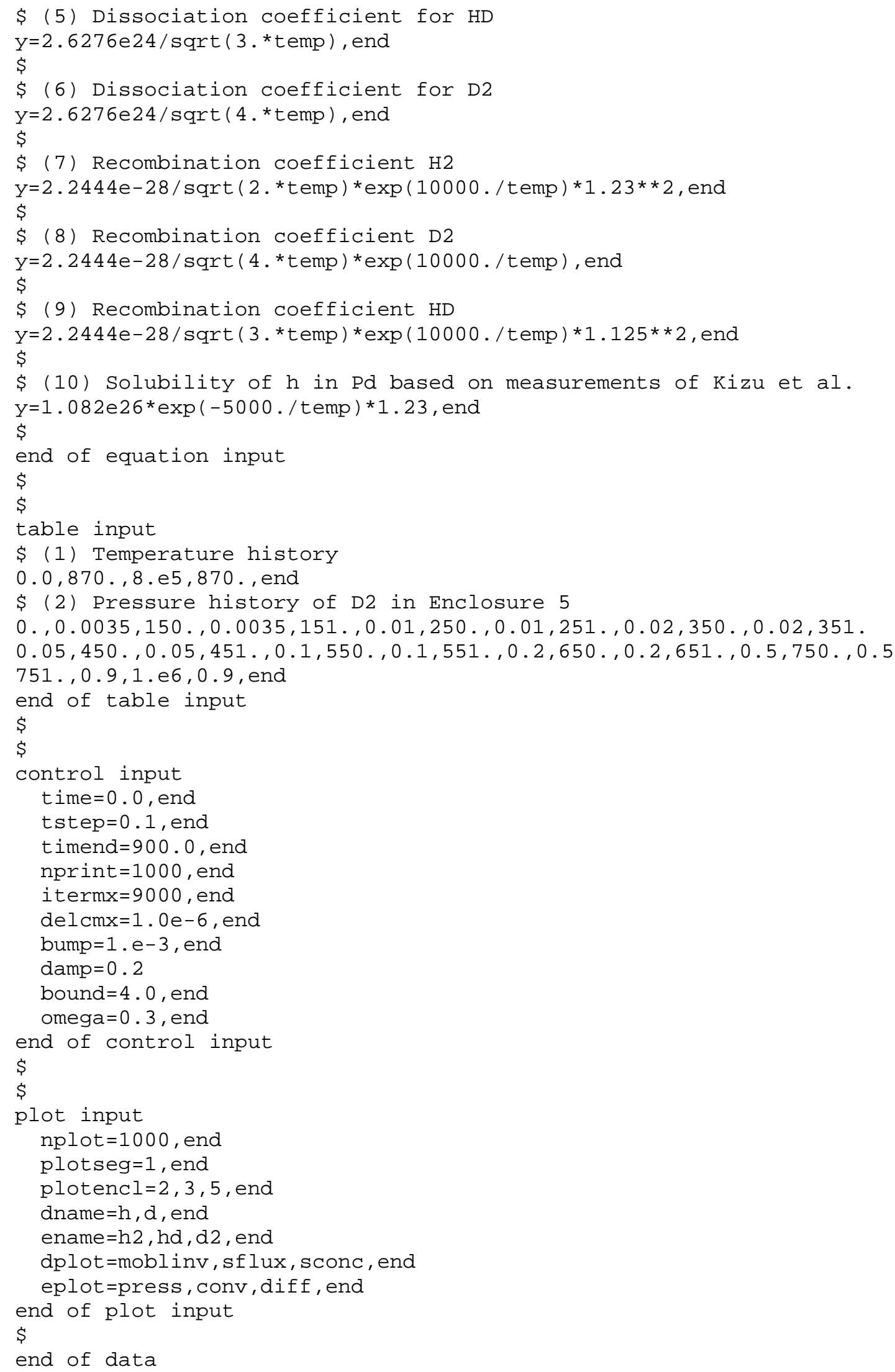


Co-permeation of H and D through Pd (Val-2eeb, see p. 53)

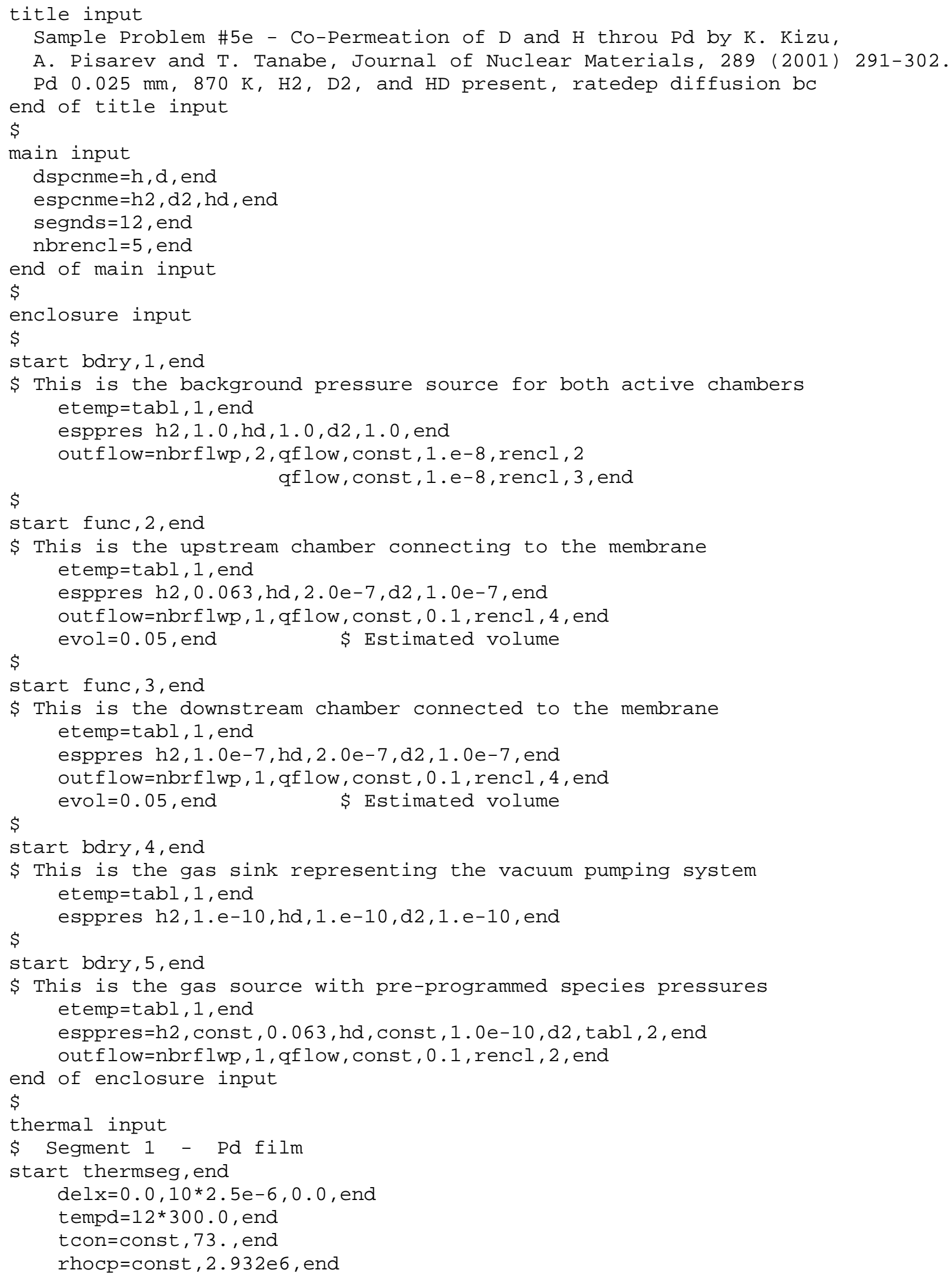




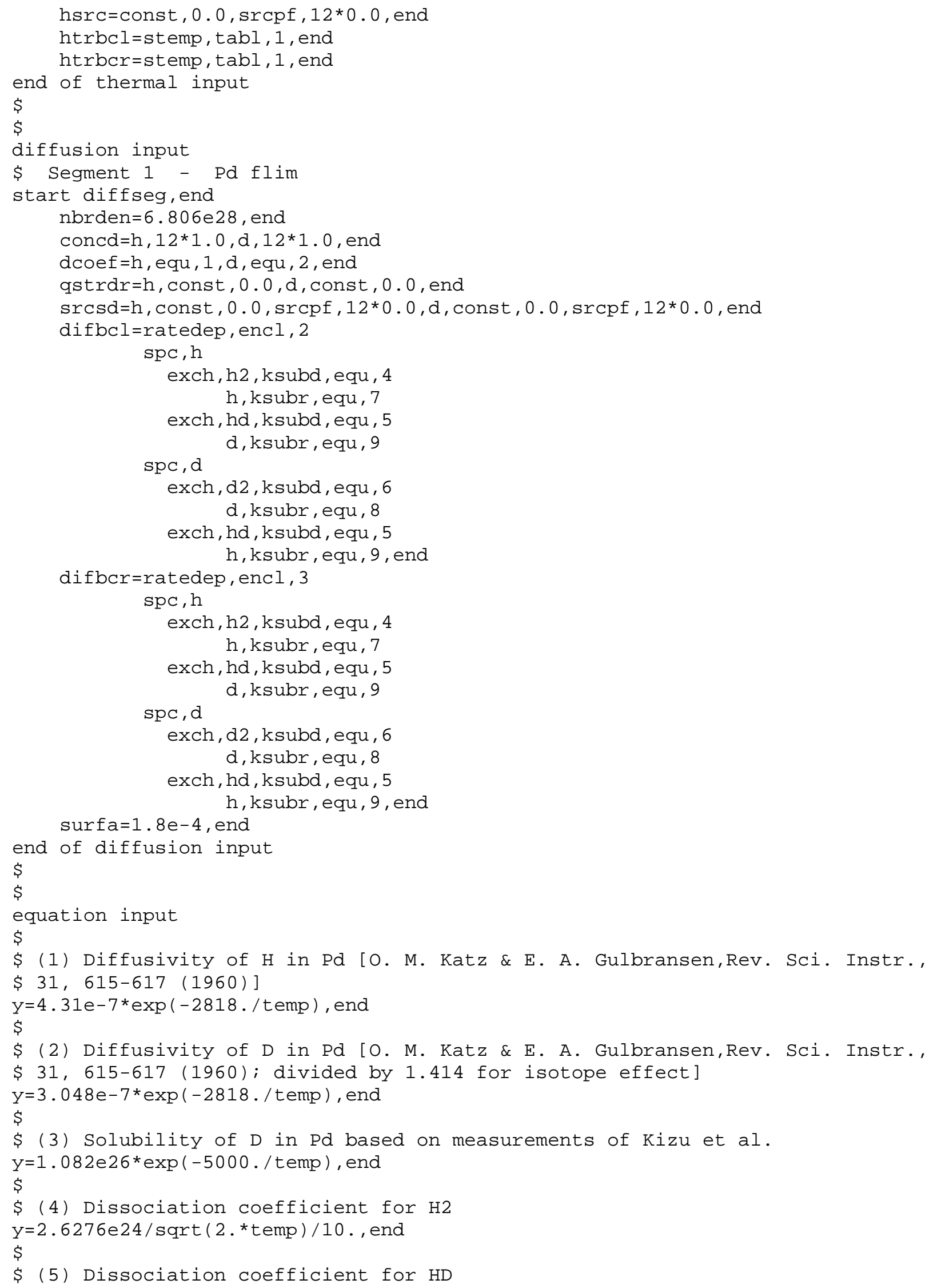




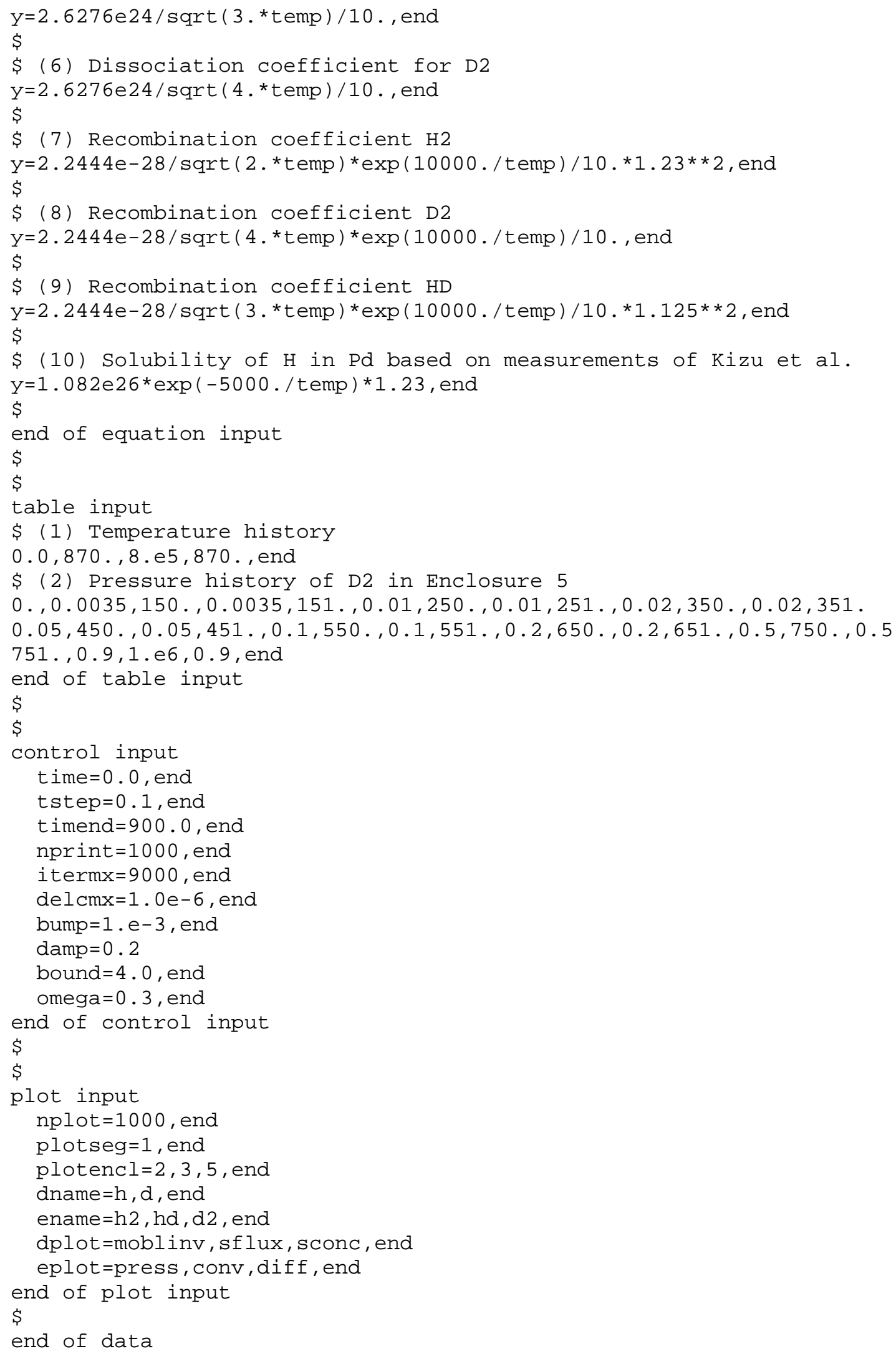


Co-permeation of H and D through Pd (Val-2ef, see p. 53)

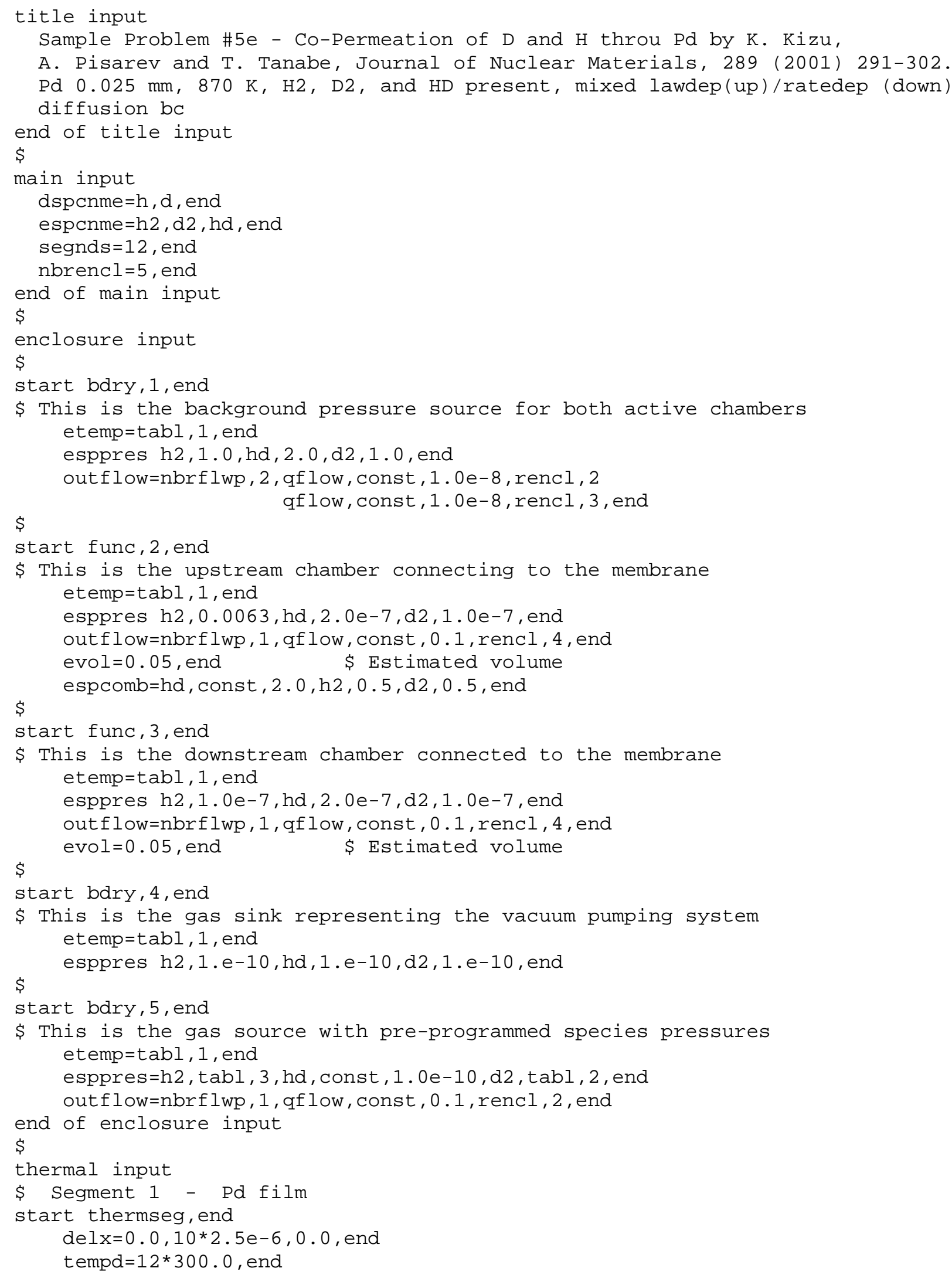




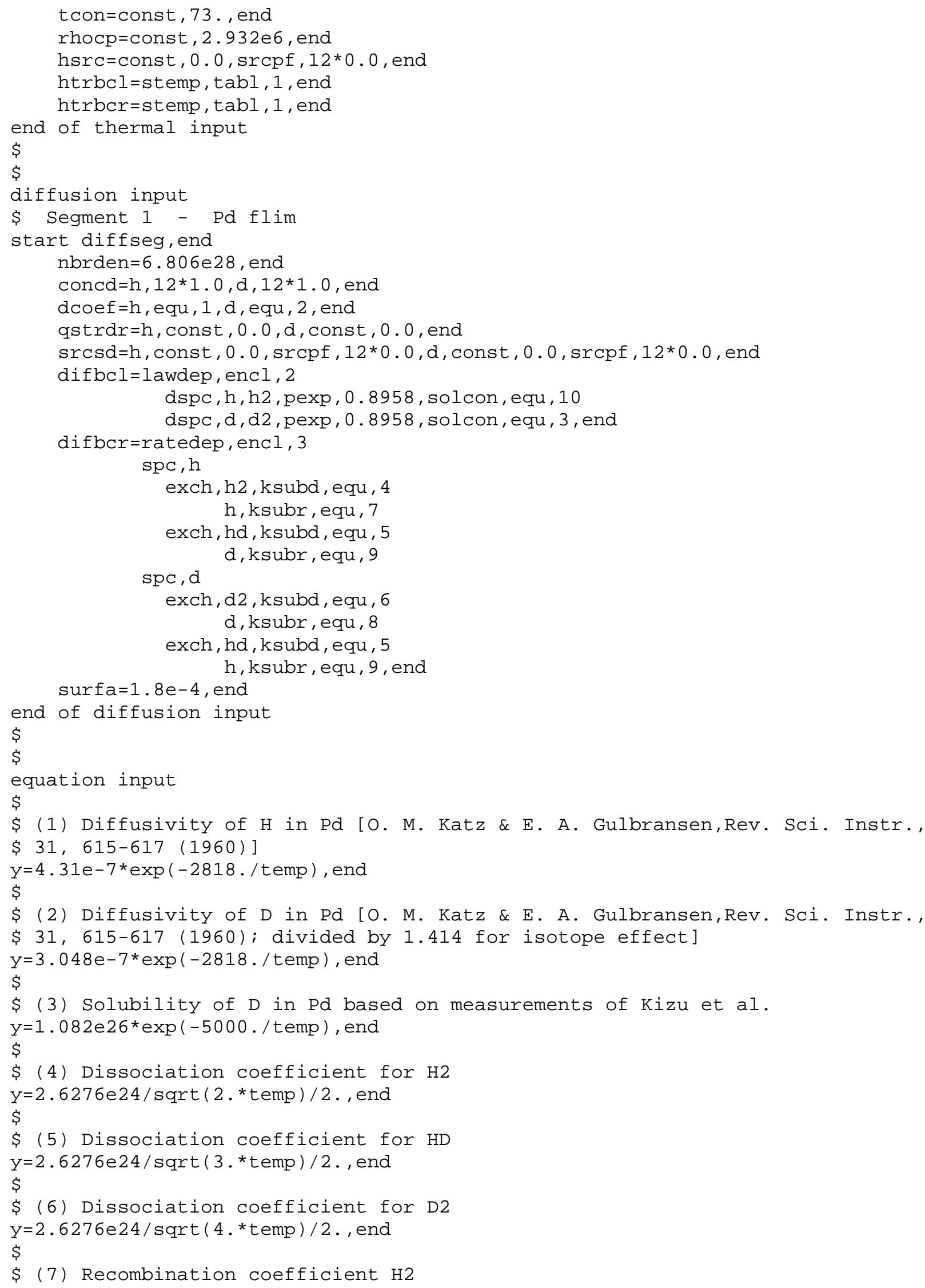




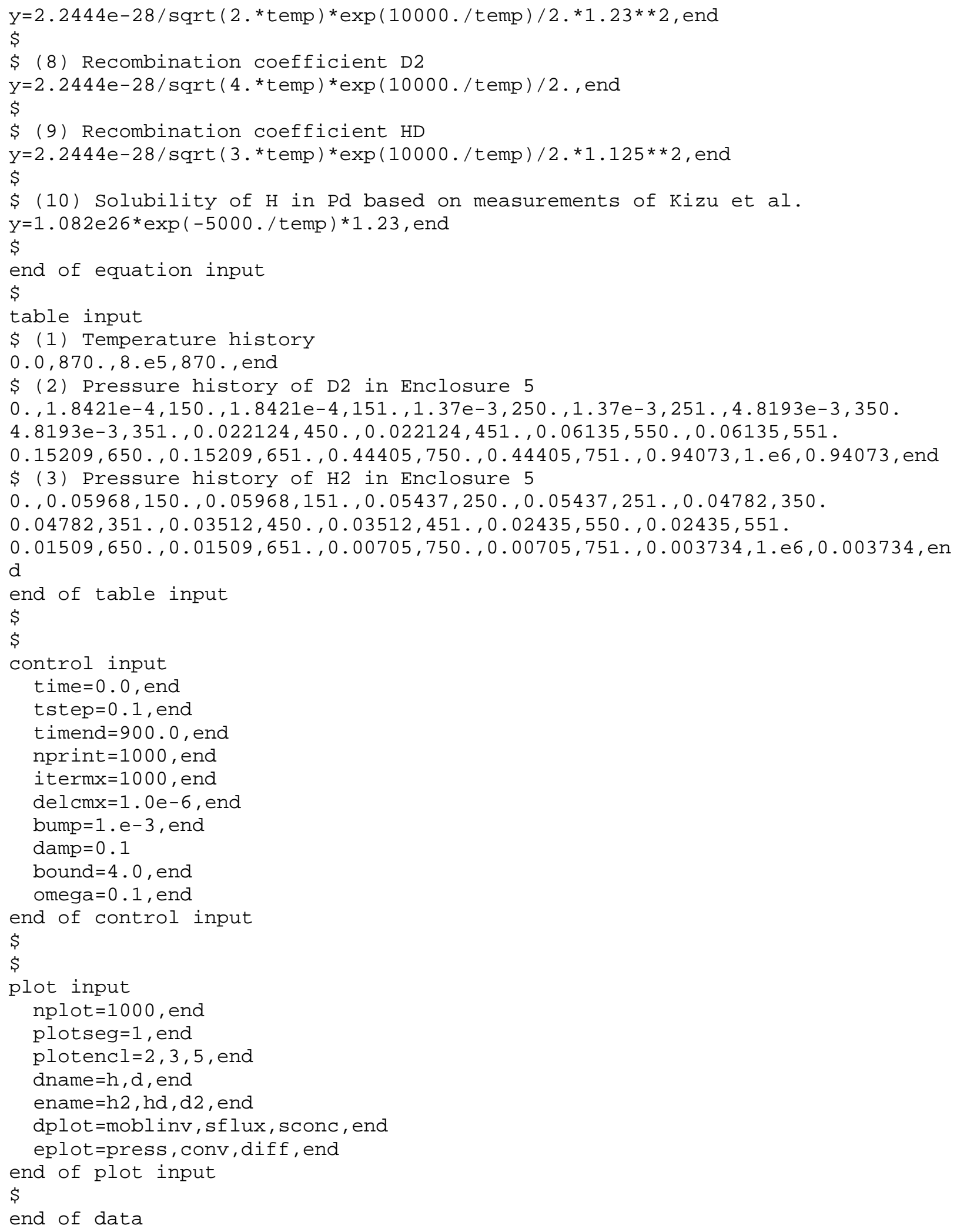

